\title{
Kreiszylinderschalen unter der Einwirkung von Einzelmomenten und radialen Einzellasten
}

Zur Erlangung des akademischen Grades eines

DOKTOR INGENIEURS

von der Fakultät für

Bauingenieur-, Geo- und Umweltwissenschaften der Universität Fridericiana zu Karlsruhe (TH)

genehmigte

DISSERTATION

von

Dipl.-Ing. Elke Feifel

aus Mutlangen

Tag der mündlichen Prüfung: $\quad$ 18.07.2007

Hauptreferent:

Prof. Dr.-Ing. H. Saal

Korreferent:

Prof. Dr.-Ing. H. Schmidt

Karlsruhe 2007 



\section{Vorwort}

Die vorliegende Arbeit entstand während meiner Tätigkeit als wissenschaftliche Mitarbeiterin am Lehrstuhl für Stahl- und Leichtmetallbau der Universität Karlsruhe (TH).

Mein ganz besondere Dank gilt Herrn Professor Dr.-Ing. Helmut Saal für die Anregung zu dieser Arbeit. Für seine wertvollen Denkanstöße, die sich in dieser Arbeit wiederfinden, und seine unermüdliche Diskussionsbereitsschaft danke ich ihm ebenso herzlich wie für die Betreuung dieser Arbeit.

Herrn Professor Dr.-Ing. Herbert Schmidt danke ich für die Übernahme des Korreferats und die kritische Durchsicht meiner Arbeit.

Allen Kollegen, Mitarbeitern und wissenschaftlichen Hilfskräften des Lehrstuhls für Stahl- und Leichtmetallbau sei weiterhin die äußerst angenehme Atmosphäre und Zusammenarbeit gedankt. Weiterhin danke ich Herrn Dr.-Ing. Tobias Loose für die Durchsicht meines Manuskripts und für die hilfreichen Diskussionen zu den Untersuchungen und Ergebnissen meiner Arbeit.

Ich danke meinen Eltern, die mir meine Ausbildung ermöglicht haben und deren tatkräftiger Unterstützung ich mir stets sicher sein konnte.

Mein ganz besonderer Dank gilt meinem Lebensgefährten Jan Horn und meiner Tochter Annika, ohne deren Unterstützung und aufmunternden Worte diese Arbeit nicht hätte gelingen können.

Ulm, im Februar 2008

Elke Feifel 



\section{Kurzfassung}

Kreiszylinderschalen sind aufgrund ihrer Nutzung zur Lagerung von gasförmigen Stoffen und Flüssigkeiten hauptsächlich Belastungen ausgesetzt, die über die Membrantragwirkung abgetragen werden. Da die Spannungen und Verformungen infolge dieser Belastungen sehr gering sind, können Kreiszylinderschalen als sehr dünnwandige Strukturen ausgeführt werden. Infolge von Stutzenlasten erfährt die Schalenwand zusätzlich zu den Membranbeanspruchungen auch Biegebeanspruchungen. Dabei entstehen große Spannungen im Anschlussbereich, die sich auf das Tragverhalten der Struktur auswirken und eine Querschnittsverstärkung in diesem Bereich erforderlich machen können. Bisherige theoretische und experimentelle Untersuchungen auf diesem Gebiet beschränkten sich auf Kreiszylinderschalen mit kleinen und mittleren $R / t$-Verhältnissen, wobei geometrische und physikalische Nichtlinearitäten außer acht gelassen wurden.

In dieser Arbeit wurden mit der FE-Methode Kreiszylinderschalen untersucht, deren $R / t$-Verhältnisse zwischen 400 und 5000 liegen. Dabei wurden Kreiszylinderschalen unter Einwirkung radialer Einzellasten, Momentenbelastung in Umfangsrichtung und in Meridianrichtung unter Berücksichtigung geometrisch und physikalisch nichtlinearer Effekte betrachtet. In den Berechnungen wurde zudem der Einfluss scheibenförmiger Verstärkungsbleche auf das Tragverhalten untersucht.

Die Auswertung der Berechnungsergebnisse erfolgte auf Basis dimensionsloser Parameter. Mit vorsichtigen Bemessungsregeln kann das Tragverhalten der lokal beanspruchten Kreiszylinderschalen abgeschätzt werden. Der Einfluss von Verstärkungsblechen am Stutzenanschluss wird mit diesen Bemessungsregeln ebenfalls berücksichtigt. Die Auswirkungen des plastischen Werkstoffverhaltens auf das Tragverhalten der Kreiszylinderschalen werden durch Abmindungskurven erfasst. 


\section{Abstract}

Cylindrical shells are mainly used for the storage of gas and fluids which cause membran stresses in the wall of the shell. Due to small stresses and small deformations caused by these loads cylindrical shells are often realized as slender structures. Nozzeles attached to the wall might cause high stresses in the vicinity of the nozzel which affect the load-displacement-relations and the ultimate loads of these structures. In some cases the wall of the shell in the vicinity of the nozzle has to be reinforced. Theoretical and experimental investigations on this field dealt with cylindrical shells with small and medium $R / t$-ratios. Geometrical and physical nonlinearities were nelected in these studies.

In this work cylindrical shells with $R / t$-ratios between 400 and 5000 were investigated by means of FEA. Cylindrical shells subjedted to radial loads and moments in circumferential and axial direction were considered. These investigations take into account the effects of geometrical and physical nonlinearities. Reinforcing pads are consideres as well.

The results are presented in graphical form. Design rules are given for cylindrical shells under local loading which also applies for reinforced shells and plastic material behaviour. 


\section{Inhaltsverzeichnis}

1 Einleitung 1

1.1 Hintergrund . . . . . . . . . . . . . . . . . . . 1

1.2 Problemstellung und Zielsetzung . . . . . . . . . . . . 1

1.3 Vorgehensweise . . . . . . . . . . . . . . 3

2 Wissensstand $\quad 5$

2.1 Definition von Stabilitätsproblemen _ . . . . . . . . . 5

2.2 Analytische und experimentelle Untersuchungen an Kugelkappen unter Einzellast . . . . . . . . . . . . . . . . . . . 6

2.2.1 Geometrie der Kugelkappe . . . . . . . . . . . . . . . 6

2.2.2 Spannungen und Verformungen von Kugelkappen nach der geometrisch linearen Theorie . . . . . . . . . . 6

2.2.3 Das Tragverhalten von Kugelkappen bei großen Verformungen . . . . . . . . . . . . . 7

2.3 Analytische und experimentelle Untersuchungen an Kreiszylinderschalen unter lokaler Belastung . . . . . . . . . . . . 13

2.4 Behandlung des Problems in Normen und Regelwerken . . . . . 18

2.5 Zusammenfassung . . . . . . . . . . . . . . 18

3 Berechnungen mit der Finiten Elemente Methode - Vorüberlegungen und Grundlagen $\quad 20$

3.1 Allgemeines . . . . . . . . . . . . . . 20

3.2 Verwendete Elemente . . . . . . . . . . . . . . . . . 20

3.2.1 Schalenelemente . . . . . . . . . . . . 20

3.2.2 Kontaktelemente . . . . . . . . . . . . . . . 20

3.3 Das FE-Modell der Kugelkappe . . . . . . . . . . . . . . . 21

3.3.1 Lasteinleitung . . . . . . . . . . . . . . . . . 21 
3.3.2 FE-Modell der Kugelkappe mit Lasteinleitung über eine Fläche . . . . . . . . . . . . . . . . . 21

3.3.3 FE-Modell der Kugelkappe mit Lasteinleitung über einen Stutzen . . . . . . . . . . . . . . 22

3.4 FE-Modell der Kreiszylinderschale . . . . . . . . . . . . . . 25

3.5 Werkstoffgesetz . . . . . . . . . . . . . 27

3.6 Lastverfolgungsalgorithmus . . . . . . . . . . . . . 28

4 Das Tragverhalten der lokal belasteten Kugelkappe 30

4.1 Hintergrund . . . . . . . . . . . . . . . . . . . 30

4.2 Analogie von Kugelkappe und elastisch gebetteter Kreisplatte . . 30

4.3 Einfluss der Geometrieparameter am Beispiel der Kugelkappe und des Stabzweischlages . . . . . . . . . . . . . . . . 32

4.4 Einfluss der Ausführung des Lasteinleitungsbereiches . . . . . . . 42

4.5 Einfluss der Symmetrierandbedingungen . . . . . . . . . . . 46

4.6 Einfluss des Stutzens . . . . . . . . . . . . . . . . . . . 48

4.7 Zusammenfassung . . . . . . . . . . . . . . . 49

5 Das Tragverhalten der lokal belasteten Kreiszylinderschale $\quad 51$

5.1 Hintergrund . . . . . . . . . . . . . . 51

5.2 Einfluss der Geometrieparameter $L^{*}$ und $R / t \ldots \ldots \ldots$. . . . 51

5.3 Einfluss des Stutzens . . . . . . . . . . . . . . . . 53

5.4 Einfluss des Abstandes der Lastangriffspunkte in Umfangsrichtung 56

5.5 Abklinglänge der Biegestörung . . . . . . . . . . . . 58

5.6 Einfluss der Randbedingungen . . . . . . . . . . . . . . 60

5.7 Interaktion von Innendruck und lokaler Belastung . . . . . . . . 62

5.8 Vergleich der numerischen Ergebnisse mit analytischen und numerischen Ergebnissen aus WRCB 297 . . . . . . . . . 63

5.9 Vergleich der numerischen Ergebnisse mit Versuchsergebnissen der „Chicago bridge and iron company“ und analytischen Ergebnissen aus WRCB 297 . . . . . . . . . . . . . . . . 67

5.10 Zusammenfassung ................ 71 


\section{Numerische Serienberechnungen von Kreiszylinderschalen unter lo-} kaler Belastung

6.1 Umfang der Untersuchungen . . . . . . . . . . . . . . . 73

6.2 Berechnungskonzepte . . . . . . . . . . . . . . . . 73

6.3 Definition der kritischen Last bei geometrisch nichtlinearen Berechnungen . . . . . . . . . . . . . . . . 74

6.4 Kreiszylinderschalen unter radialen Einzellasten $P_{x} \ldots \ldots$. . . 76

6.4 .1 Allgemeines . . . . . . . . . . . . 76

6.4.2 Geometrisch lineare Berechnungen mit elastischem Werkstoffgesetz $\left(P_{x} \mathrm{LA}\right) \ldots \ldots \ldots$

6.4.3 Geometrisch nichtlineare Berechnungen mit elastischem Werkstoffgesetz $\left(P_{x}\right.$ GNA $) \ldots \ldots \ldots$

6.4.4 Geometrisch lineare Berechnungen unter Berücksichtigung des plastischen Werkstoffgesetzes $\left(P_{x}\right.$ MNA) $\ldots$. 87

6.4.5 Geometrisch nichtlineare Berechnungen unter Berücksichtigung des plastischen Werkstoffgesetzes ( $P_{x}$ GMNA) 90

6.4.6 Interaktion von geometrischer und materieller Nichtlinearität . . . . . . . . . . . . . . . . . . 994

6.5 Kreiszylinderschalen unter Momentenbelastung in Umfangsrichtung $M_{\varphi} \ldots \ldots \ldots \ldots \ldots \ldots \ldots$

6.5 .1 Allgemeines . . . . . . . . . . . 96

6.5.2 Geometrisch lineare Berechnungen mit elastischem Werkstoffgesetz $\left(M_{\varphi} \mathrm{LA}\right) \ldots \ldots \ldots$

6.5.3 Geometrisch nichtlineare Berechnungen mit elastischem Werkstoffgesetz $\left(M_{\varphi}\right.$ GNA $) \ldots \ldots$

6.5.4 Geometrisch lineare Berechnungen mit plastischem Werkstoffgesetz $\left(M_{\varphi}\right.$ MNA $) \ldots \ldots . \ldots 106$

6.5.5 Geometrisch nichtlineare Berechnung mit plastischem Werkstoffgesetz $\left(M_{\varphi}\right.$ GMNA) $\ldots \ldots \ldots 110$

6.5.6 Interaktion von geometrischer und materieller Nichtlinea-

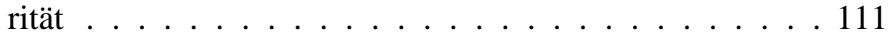

6.6 Kreiszylinderschalen unter Momentenbelastung $M_{z}$ in Meridian-

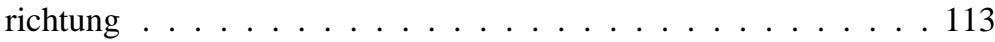
6.6 .1 Allgemeines . . . . . . . . . . . 113 
6.6.2 Geometrisch lineare Berechnungen mit elastischem Werkstoffgesetz $\left(M_{z} \mathrm{LA}\right) \ldots \ldots . \ldots 114$

6.6.3 Geometrisch nichtlineare Berechnungen mit elastischem Werkstoffgesetz $\left(M_{z} \mathrm{GNA}\right) \ldots \ldots$

6.6.4 Geometrisch lineare Berechnung mit plastischem Werkstoffgesetz $\left(M_{z}\right.$ MNA $) \ldots \ldots . \ldots 123$

6.6.5 Geometrisch nichtlineare Berechnung mit plastischem Werkstoffgesetz $\left(M_{z}\right.$ GMNA) $\ldots \ldots$. . . . . 127

6.6.6 Interaktion von geometrischer und materieller Nichtlinearität . . . . . . . . . . . . . . . . . 128

6.7 Zusammenfassung der Ergebnisse . . . . . . . . . . . . . . . . . 129

7 Verstärkung der Kreiszylinderschale im Stutzenbereich 130

7.1 Varianten der Verstärkungsmaßnahmen . . . . . . . . . . . 130

7.1.1 Hintergrund . . . . . . . . . . . . . . . . . . 130

7.1.2 Aufgesetzte Verstärkungsbleche . . . . . . . . . . 130

7.1.3 Eingesetzte Verstärkungsbleche . . . . . . . . . . 132

7.1.4 Einfluss unterschiedlicher Ausführungen von Verstärkungsblechen auf den Spannungsverlauf . . . . . . . 134

7.2 Kreiszylinderschalen mit Verstärkungsblechen unter radialen Ein-

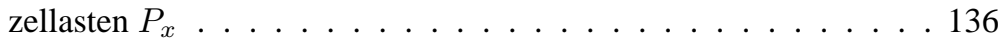

7.2.1 Geometrisch lineare Berechnungen mit elastischem Werkstoffgesetz $\left(P_{x}\right.$ ver LA) $\ldots \ldots$. . . . . . . 136

7.2.2 Geometrisch nichtlineare Berechnungen mit elastischem

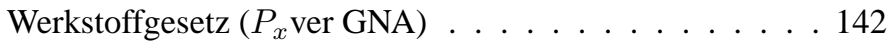

7.2.3 Einfluss materieller Nichtlinearität auf das Tragverhalten verstärkter Kreiszylinderschalen . . . . . . . . . . . 150

7.3 Kreiszylinderschalen mit Verstärkungsblechen unter Momentenbelastung $M_{\varphi}$ in Umfangsrichtung . . . . . . . . . . 152

7.3.1 Geometrisch lineare Berechnungen mit elastischem Werkstoffgesetz $\left(M_{\varphi}\right.$ ver LA) $\ldots \ldots \ldots 152$

7.3.2 Geometrisch nichtlineare Berechnungen mit elastischem Werkstoffgesetz $\left(M_{\varphi}\right.$ ver GNA $) \ldots \ldots 157$ 
7.3.3 Einfluss materieller Nichtlinearität auf das Tragverhalten verstärkter Kreiszylinderschalen . . . . . . . . . . . . 162

7.4 Kreiszylinderschalen mit Verstärkungsblechen unter Momentenbelastung $M_{z}$ in Meridianrichtung . . . . . . . . . . . 164

7.4.1 Geometrisch lineare Berechnungen mit elastischem Werkstoffgesetz $\left(M_{z}\right.$ ver LA) . . . . . . . . . . . . 164

7.4.2 Geometrisch nichtlineare Berechnungen mit elastischem Werkstoffgesetz $\left(M_{z}\right.$ ver GNA) . . . . . . . . . 168

7.4.3 Der Einfluss materieller Nichtlinearität auf das Tragverhalten verstärkter Kreiszylinderschalen . . . . . . . . 176

7.5 Zusammenfassung der Ergebnisse . . . . . . . . . . . . . 176

8 Anwendungsbeispiele

8.1 Tank mit einem Durchmesser von $8 \mathrm{~m}$ unter Einwirkung von $P_{x}$ und $M_{\varphi} \ldots \ldots \ldots \ldots \ldots \ldots \ldots \ldots$

8.2 Tank mit einem Durchmesser von 20 m unter Einwirkung von $P_{x} .184$

9 Zusammenfassung

Schrifttum 


\section{Indizes}

$\begin{array}{ll}\text { crit } & \text { kritisch } \\ \text { GMNA } & \text { geometrisch und physikalisch nichtlinear } \\ \text { GNA } & \text { geometrisch nichtlinear } \\ \mathrm{i} & \text { Lastfall } \\ \mathrm{j} & \text { Laufvariable } \\ \mathrm{k} & \text { Berechnungsart } \\ \text { LA } & \text { geometrisch und physikalisch linear } \\ \text { max } & \text { Maxium } \\ \min & \text { Minimum } \\ \text { MNA } & \text { physikalisch nichtlinear } \\ \mathrm{r} & \text { Stutzen } \\ \text { ver } & \text { Verstärkung }\end{array}$

\section{Bezeichnungen}

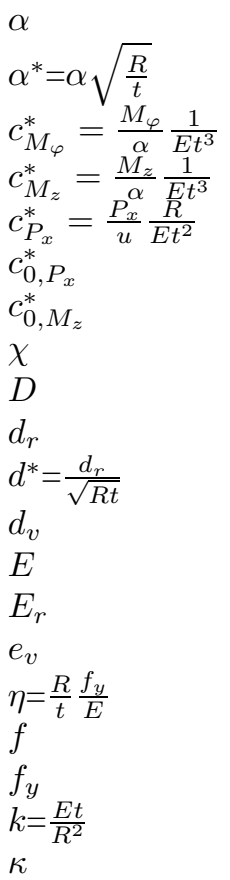

Verdrehung des Stutzens

dimensionslose Verdrehung des Stutzens

dimensionslose Anfangssteifigkeit (Lastfall $M_{\varphi}$ )

dimensionslose Anfangssteifigkeit (Lastfall $M_{z}$ )

dimensionslose Anfangssteifigkeit (Lastfall $P_{x}$ )

Hilfswert zur Bestimmung von $c_{P_{x}}^{*}$

Hilfswert zur Bestimmung von $c_{M_{z}}^{*}$

Hilfswert zur Bestimmung von $c_{P_{x}}^{*}$

Durchmesser der Schale

Stutzendurchmesser

dimensionsloser Stutzendurchmesser

Verstärkungsblechdurchmesser

Elastizitätsmodul

Elastizitätsmodul des Stutzens

Exzentrizität des Verstärkungsbleches

plastischer Abgrenzungsfaktor

Stich der Kugelkappe

Streckgrenze

Bettungsziffer

Krümmung 


\begin{tabular}{|c|c|}
\hline$\lambda=\sqrt[4]{\frac{12 L^{4}\left(1-\nu^{2}\right)}{R^{2} t^{2}}}$ & Schlankheitsparameter der Kugelkappe \\
\hline$L=\frac{L}{L^{*}}$ & $\begin{array}{l}\text { Abstand des Lastangriffspunktes zum Schalenrand } \\
\text { dimensionsloser Randabstand des Lastangriffs- } \\
\text { punktes (Kreiszylinderschale) }\end{array}$ \\
\hline$l_{r}$ & Stutzenlänge \\
\hline$l_{r}^{*}=\frac{l_{r}}{\sqrt{d_{r} t_{r} / 2}}$ & dimensionslose Stutzenlänge \\
\hline$l_{v}^{*}=\frac{l_{v}}{\sqrt{R t_{v, e f f}}}$ & $\begin{array}{l}\text { Länge des Verstärkungsbleches } \\
\text { dimensionslose Verstärkungsblechlänge }\end{array}$ \\
\hline$l_{v, \min }^{*}$ & Mindestlänge des Verstärkungsbleches \\
\hline$\mu$ & Reibkoeefizient \\
\hline$M_{\varphi}$ & Moment in Umfangsrichtung \\
\hline$M_{\varphi}^{*}=\frac{M_{\varphi}}{E t^{3}} \sqrt{\frac{R}{t}}$ & dimensionsloses Moment in Umfangsrichtung \\
\hline$M_{\varphi, \text { crit }}^{*}$ & $\begin{array}{l}\text { dimensionsloses kritisches Moment in Umfangs- } \\
\text { richtung }\end{array}$ \\
\hline $\bar{M}_{\varphi}=\frac{M_{\varphi}}{f_{y} t^{2} \sqrt{R t}}$ & dimensionsloses plastisches Moment in Umfangs- \\
\hline $\begin{array}{l}\bar{M}_{\varphi, \max } \\
M_{z, 0}^{*} \\
M_{z}\end{array}$ & $\begin{array}{l}\text { richtung } \\
\text { dimensionsloses plastisches Grenzmoment } \\
\text { Hilfswert zur Bestimmung von } M_{z, \text { crit }, \text { GNA,ver }} \\
\text { Moment in Meridianrichtung }\end{array}$ \\
\hline$M_{z}^{*}=\frac{M_{z}}{E t^{3}} \sqrt{\frac{R}{t}}$ & dimensionsloses Moment in Meridianrichtung \\
\hline$M_{z, \text { crit }}^{*}$ & $\begin{array}{l}\text { dimensionsloses kritisches Moment in Meridian- } \\
\text { richtung }\end{array}$ \\
\hline $\bar{M}_{z}=\frac{M_{z}}{f_{y} t^{2} \sqrt{R t}}$ & dimensionsloses plastisches Moment in \\
\hline $\bar{M}_{z, \max }$ & $\begin{array}{l}\text { Meridianrichtung } \\
\text { dimensionsloses plastisches Grenzmoment in } \\
\text { Meridianrichtung }\end{array}$ \\
\hline$\nu$ & Querdehnzahl \\
\hline$\omega$ & Hilfswert zur Bestimmung von $c_{M_{z}}^{*}$ \\
\hline$p_{i}$ & Radialdruck des Füllmediums \\
\hline$P_{0}^{*}$ & Hilfswert zur Bestimmung von $P_{x, \text { crit }, G N A, \text { ver }}$ \\
\hline$P_{x}$ & Last in radialer Richtung \\
\hline$P_{x}^{*}=\frac{P R}{E t^{3}}$ & dimensionslose Last \\
\hline$P_{x, \text { crit }}^{*}$ & kritische Last in radialer Richtung \\
\hline $\bar{P}_{x}=\frac{P}{f_{y} t^{2}}$ & dimensionslose plastische Last \\
\hline $\bar{P}_{x, \max }$ & dimensionslose plastische Grenzlast \\
\hline 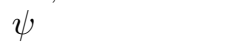 & zur Bestimmung von $P_{x, c r i t, G N A, v e r}$ \\
\hline
\end{tabular}




$\begin{array}{ll}R & \text { Schalenradius } \\ \sigma_{\varphi} & \text { Umfangsspannung } \\ \sigma_{z} & \text { Längsspannung } \\ \sigma_{\varphi, P_{x}}^{*}=\sigma \frac{t^{2}}{P_{x}} & \text { dimensionslose Umfangsspannung infolge } P_{x} \\ \sigma_{\varphi, \text { max }, P_{x}}^{*} & \text { maximale dimensionslose Umfangsspannung } \\ \sigma_{z, P_{x}}^{*}=\sigma \frac{t^{2}}{P_{x}} & \text { infolge } P_{x} \\ \sigma_{z, M_{\varphi}}^{*}=\sigma \frac{d_{r} t^{2}}{M_{\varphi}} & \text { dimensionslose Längsspannung infolge } P_{x} \\ \sigma_{z, \text { max }, M_{\varphi}}^{*} & \text { dimensionslose Längsspannung infolge } M_{\varphi} \\ & \text { maximale dimensionslose Längsspannung } \\ \sigma_{\varphi, M_{z}}^{*}=\sigma \frac{d_{r} t^{2}}{M_{z}} & \text { infolge } M_{\varphi} \\ \sigma_{\varphi, \text { max }, M_{z}}^{*} & \text { dimensionslose Umfangsspannung infolge } M_{z} \\ s_{\varphi} & \text { maximale dimensionslose Umfangsspannung } \\ t & \text { infolge } M_{z} \\ t_{p} & \text { Bogenlänge in Umfangsrichtung } \\ t_{r} & \text { Wanddicke der Schale } \\ t_{v} & \text { Wanddicke im Lasteinleitungsbereich einer Kugelkappe } \\ t_{v, \text { eff }} & \text { Wanddicke des Stutzens } \\ u & \text { Dicke des Verstärkungsbleches } \\ u^{*}=\frac{u}{t} & \text { effektive Verstärkungsblechdicke } \\ v & \text { Verformung in radialer Richtung } \\ \xi & \text { dimensionslose radiale Verformung } \\ x & \text { Verformung in tangentialer Umfangsrichtung } \\ \varphi & \text { Hilfswert zur Bestimmung von } M_{z, c r i t, G N A, v e r} \\ z & \text { Koordinate in radialer Richtung der Kreiszylinder- } \\ & \text { schale } \\ & \text { Koordinate in tangentialer Richtung der Kreis- } \\ & \text { zylinderschale } \\ & \text { Koordinate in Längsrichtung der Kreiszylinderschale } \\ & \end{array}$




\section{Einleitung}

\subsection{Hintergrund}

Große Behälter, die der Lagerung flüssiger und gasförmiger Stoffe dienen, werden oft in Form von Kreiszylindern ausgeführt. Da die Reibung zwischen dem flüssigen oder gasförmigem Lagergut und der Wand vernachlässigbar klein ist, wird die Wand hauptsächlich durch Umfangszugspannungen beansprucht, die durch den hydrostatischen Druck der Flüssigkeit oder den Innendruck des gasförmigen Lagergutes entstehen. Die Lastabtragung erfolgt dabei über die Membrantragwirkung in Umfangsrichtung. Die Spannungen und Verformungen infolge dieser Belastungen sind sehr gering und ermöglichen die Ausführung sehr dünnwandiger Strukturen. Aus dem Eigengewicht des Mantels und bei geschlossenen Behältern aus dem Eigenwicht des Daches entstehen Axialdruckspannungen, die über Membrankräfte in Längsrichtung abgetragen werden. Aufgrund der Axialdruckspannungen und Umfangsdruckspannungen, wie sie beispielsweise aus Unterdruck über dem Füllgut oder Winddruck enstehen, sind die sehr dünnwandigen Kreiszylinderschalen stabilitätsgefährdet. Die Druckspannungen aus Axial- oder Radialdruck können daher auch die erforderliche Dicke des Mantelbleches bestimmen. Bei großen Tankbauwerken, wie sie beispielsweise zur Lagerung von Erdöl eingesetzt werden, kann das Verhältnis des Tankradius $R$ zur Dicke $t$ des Mantelbleches durchaus im Bereich von bis zu 5000 liegen.

\subsection{Problemstellung und Zielsetzung}

Zur Beförderung von Lagergut zwischen, zu und von Tankbauwerken werden Rohrleitungen eingesetzt, die häufig über Stutzen an die Behälterwand angeschlossen sind (Abbildung 1.1). Infolge Temperaturdehnung oder Lagergutbewegungen in den Rohrleitungen kommt es zur lokalen Beanspruchung der Schalenwand. Zusätzlich zu den Membranbeanspruchungen erfährt die Schalenwand in solchen Fällen auch Biegungbeanspruchungen. Dabei entstehen große Spannungen im Anschlussbereich, die sich auf das Tragverhalten der Struktur 
auswirken und eine Querschnittsverstärkung in diesem Bereich erforderlich machen können. Die dabei auftretenden Verformungen können auch bei geringen Lasten ein vielfaches der Wanddicke betragen und die Gebrauchstauglichkeit sowie das optische Erscheinungsbild des Tankbauwerks beeinflussen. Im Folgenden wird die Bezeichnung Stutzen sowohl für rohrförmige Hilfskonstruktionen zum Anschluss der Rohre am Behälter als auch für unmittelbar an den Behälter anschließdende Rohrabschnitte verwendet.

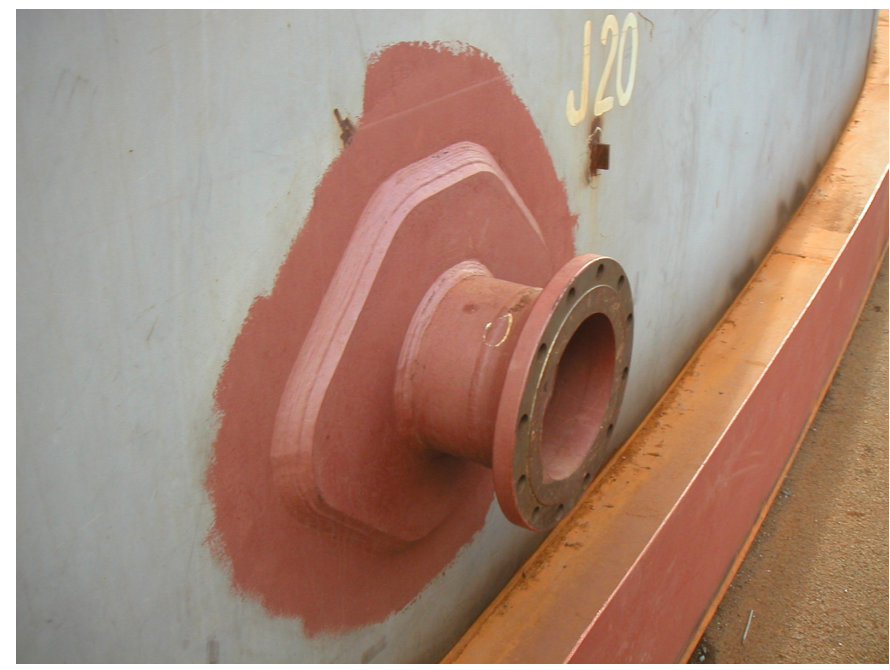

Abbildung 1.1: Tank mit Stutzen

Die durch lokale Belastungen bedingten Spannungsspitzen können dazu führen, dass die Schalenwand oder der Stutzen plastizieren. Diese Gefahr besteht insbesondere bei Kreiszylinderschalen mit kleinem $R / t$-Verhältnis, da aufgrund der größeren Steifigkeit die Zwängungskräfte deutlich größer sind als bei sehr dünnwandigen Strukturen. Weiterhin können häufige Lastwechsel zur Ermüdung der Konstruktion führen. Bei Kreiszylinderschalen mit großen $R / t$-Verhältnissen spielen dagegen geometrische Nichtlinearitäten eine weitaus wichtigere Rolle als das plastische Werkstoffverhalten. Druckspannungen in der Behälterwand infolge der lokalen Belastungen können bei schlanken Bauwerken zu einem Stabilitätsproblem führen.

Bisherige theoretische und experimentelle Untersuchungen auf diesem Gebiet beschränkten sich auf Kreiszylinderschalen mit kleinen und mittleren 
$R / t$-Verhältnissen. Zudem war bei den untersuchten Kreiszylinderschalen das Verhältnis des Stutzendurchmessers zum Durchmesser der Schale, d.h. das $d_{r} / D$-Verhältnis, oft sehr groß. Bei den analytischen Lösungen wurde elastisches Werkstoffverhalten vorausgesetzt. Die experimentell untersuchten Kreiszylinderschalen verhielten sich ebenfalls größtenteils elastisch. Zudem wurden in den Untersuchungen keine wesentlichen geometrischen Nichtlinearitäten berücksichtigt, und es herrscht Unklarheit über den Einfluss von Verstärkungsmaßnahmen im lokal beanspruchten Bereich.

Anliegen dieser Arbeit ist es daher, das Tragverhalten von Kreiszylinderschalen mit großen $R / t$-Verhältnissen unter lokalen Belastungen systematisch zu untersuchen. Dabei werden neben geometrischen Nichlinearitäten auch die Einflüsse des plastischen Werkstoffverhaltens auf das Tragverhalten betrachtet. Auf den Einfluss von Verstärkungsblechen im lokal beanspruchten Bereich wird in dieser Arbeit ebenfalls eingegangen. Es werden stählerne Kreiszylinderschalen unter Einwirkung von radialen Einzellasten und Momenten in Umfangs- und Meridianrichtung untersucht. Die Untersuchungen werden mit der Methode der Finiten Elemente (FEM) durchgeführt. Ziel ist es, für in der Baupraxis relevante Abmessungsverhältnisse und Ausführungsvarianten Parameterstudien durchzuführen und anhand der Ergebnisse ein Abgrenzungskriterium der Tragfähigkeit abzuleiten. Unter Einbeziehung der maßgebenden Parameter und Einflussfaktoren werden schließlich Regeln vorgeschlagen, die das Tragverhalten einer Kreiszylinderschale unter lokaler Belastung beschreiben.

\subsection{Vorgehensweise}

Der zusammengetragene Wissensstand über Kreiszylinderschalen unter lokaler Belastung beschreibt deren Tragverhalten in Abhängigkeit von Geometrieparametern und Randbedingungen. Gleichzeitig werden dabei auch Wissenslücken im Bereich großer $R / t$-Verhältnisse und kleiner $d_{r} / D$-Verhältnisse aufgezeigt. Zum generellen Verständnis des Tragverhaltens dünnwandiger Strukturen unter lokaler Belastung werden zunächst Kugelkappen unter einer im Scheitel angreifenden Einzellast betrachtet, da diese Strukturen ein ähnliches Verhalten wie Kreiszylinderschalen unter lokaler Belastung aufweisen. Im Hinblick auf die Verifizierung der FE-Modelle erweist sich die Verfügbarkeit analytischer Lösungen und Versuchsergebnisse für dünnwandige Kugelkappen als entscheidender Vorteil. Die Analogiebetrachungen mit einfachen Systemen, wie dem Stabzweischlag, verdeutlichen den Einfluss der einzelnen Parameter auf das Tragverhalten 
der lokal beanspruchten Kugelkappen. Die für die Kugelkappe gewonnenen Erkenntnisse sind dabei größtenteils auf die Kreiszylinderschale übertragbar.

Da die folgenden Serienberechnungen ein effizientes FE-Modell erfordern, sind Idealisierungen und Vereinfachungen bei der Abbildung der Kreiszylinderschalen genau zu prüfen und abzuwägen. Neben der Wahl geeigneter Elemente und Berechnungsalgorithmen spielt vor allem eine sinnvolle Umsetzung der Konstruktionsdetails und der Randbedingungen der Struktur eine entscheidende Rolle. Diesen Fragen wird nachgegangen, indem das grundsätzliche Tragverhalten am Beispiel einer Kreiszylinderschale unter radialen Einzellasten untersucht wird. Anhand von Vergleichsberechnungen werden Modellannahmen und Vereinfachungen verifiziert. Ziel der Voruntersuchungen ist es, zum einen ein effizientes FE-Modell für die Serienberechnungen zu erzeugen, zum anderen den Untersuchungsumfang hinsichtlich der Parameter festzusetzen. In den Serienberechnungen werden schließlich stählerne Kreiszylinderschalen unter radialer Einzellast sowie unter Momentenbelastung in Umfangs- und Meridianrichtung betrachtet. Dabei werden geometrische Nichtlinearitäten und plastisches Werkstoffverhalten berücksichtigt. Desweiteren ist von Interesse, inwiefern sich eine Verstärkung des Stutzenanschlußbereiches auf das Tragverhalten der lokal beanspruchten Kreiszylinderschalen auswirkt. Die Auswertung entsprechender Berechnungsergebnisse erfolgt auf Basis dimensionsloser Parameter und zielt auf eine vorsichtige Bemessungsregel ab. 


\section{Wissensstand}

\subsection{Definition von Stabilitätsproblemen}

Nach innen gerichtete Einzellasten erzeugen bei Kugelkappen und Kreiszylinderschalen Druckspannungen, die zu einem Stabilitätsproblem führen können. Der Verlauf der Last-Verformungskurve eines Systems gibt Aufschluss darüber, ob ein Stabilitäts- oder ein Spannungsproblem vorliegt. Beim Spannungsproblem hat die Last-Verformungskurve einen stetigen und monoton steigenden Verlauf. Der Zusammenhang zwischen Last- und Verschiebungszustand ist eindeutig. Eine Höchstlast ist in diesem Fall erreicht, wenn eine bestimmte Verformung oder eine Grenzspannung überschritten ist. Ein Stabilitätsproblem liegt vor, wenn zu einem bestimmten Wert der Belastung mehr als ein Verformungszustand möglich ist. Beim Stabilitätsproblem wird wiederum zwischen Durchschlag- und Verzweigungsproblem unterschieden. Der typische Last-Verformungsverlauf eines Durchschlagproblems ist in Abbildung 2.1 (a), der eines Verzweigungsproblems in Abbildung 2.1 (b) dargestellt.

(a)

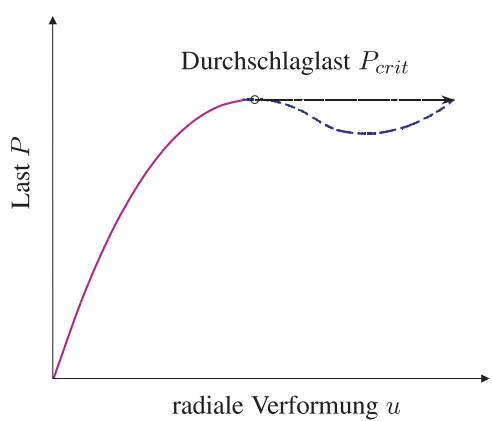

(b)

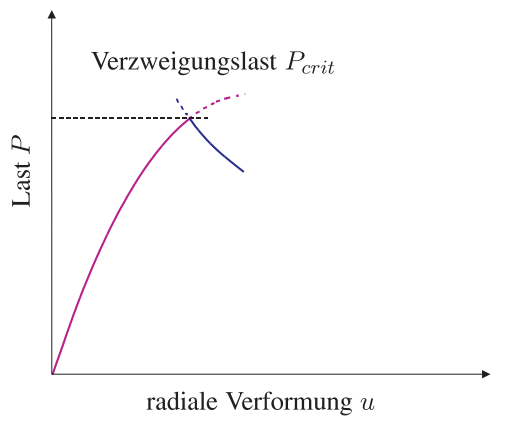

Abbildung 2.1: (a) Durchschlagproblem und (b) Verzweigungsproblem

Im Durchschlagpunkt ist die Tangente an die Last-Verformungskurve im Grundzustand horizontal. Die kritische Last $P_{\text {crit }}$ ist im Durchschlagpunkt erreicht, und 
es stellt sich auf gleichem Lastniveau bei einem anderen, entfernten Formänderungszustand ein stabiles Gleichgewicht ein. Beim Verzweigungroblem ist die Tangente an die Last-Verformungskurve im Grundzustand im Verzweigungspunkt von Null verschieden.

\subsection{Analytische und experimentelle Untersuchun- gen an Kugelkappen unter Einzellast}

\subsubsection{Geometrie der Kugelkappe}

Die Kugelkappen unter einer im Scheitel angreifenden Einzellast $P$ nach Abbildung 2.2 waren Gegenstand zahlreicher analytischer und experimenteller Untersuchungen. Diese Kugelkappen werden durch die Schlankheit

$$
\lambda=\sqrt[4]{12\left(1-\nu^{2}\right)} \frac{L}{\sqrt{R t}}
$$

und das $R / t$-Verhältsniss in dimensionsloser Form beschrieben. Bei der Lagerung der Ränder wird zwischen vertikal gelagerten (Abbildung 2.2(a)) und eingespannten Kugelkappen (Abbildung 2.2(b)) unterschieden.

\subsubsection{Spannungen und Verformungen von Kugelkappen nach der geometrisch linearen Theorie}

Reissner veröffentlichte in den Jahren 1946 bis 1950 drei Arbeiten ([1], [2] und [3]), in denen er zwei voneinander unabhängige Differentialgleichungn der dünnen Kugelkappe sowie eine geschlossene Lösung für den rotationssymmetrischen Lastfall der radialen Einzellast formulierte. Mit Reissners Gleichungen werden nichtlineare Effekte, die sich bei großen Verformungen einstellen, nicht berücksichtigt. Deshalb sind diese Gleichungen nur für flache Kugelkappen anwendbar, deren Höhe maximal 1/4 des Grundkreisradius beträgt.

Ausgehend von Reissners Differentialgleichungn veröffentlichte Bijlaard 1957 in [4] und [5] geschlossene Lösungen zur Ermittlung der Spannungen und Verformungen in Kugelkappen, die durch eine radiale Last beansprucht werden. Vereinfachend wurde dabei von einem vollkommen steifen, in die Kugelkappe eingesetzten zylinderischen Stutzen ausgegagen. In den darauffolgenden Jahren veröffentlichte Bijlaard in [6] und [7] Untersuchungen, in denen er auch die Nachgiebigkeit des Stutzens berücksichtigte. Die Ergebnisse dieser Arbeiten 
(a)

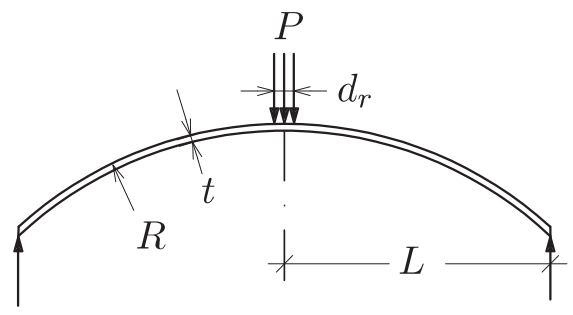

(b)

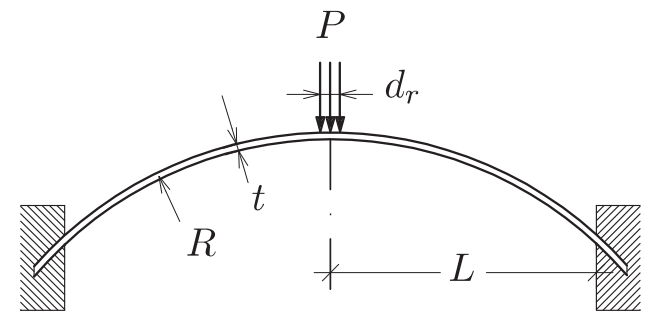

Abbildung 2.2: Kugelkappe unter Einzellast $P$; (a) nur vertikal gelagert und (b) eingespannt

werden im Welding research council bulletin (WRCB) 107 [8] zusammengefasst, in dem die Spannungen als Kurven in Abhängigkeit von den dimensionslosen Abmessungen der Kugelkappen dargestellt sind.

\subsubsection{Das Tragverhalten von Kugelkappen bei großen Verfor- mungen}

Bei Kugelkappen unter einer lokalen Belastung ist die lineare Theorie nur für schwach gekrümmte Strukturen zutreffend. Für sehr schlanke Kugelkappen und im Bereich großer Verformungen können die nichtlinearen Effekte nicht mehr vernachlässigt werden. Beim nichtlinearen Problem kann es zum Durchschlagen der Kugelkappe oder zum Auftreten eines Verzweigungsproblems nach Abschnitt 2.1 kommen. Dabei können oberhalb des ersten Verzweigungspunktes weitere Verzweigungspunkte auftreten, die mit asymmetrischen Beulformen einhergehen. Als kritische Last $P_{\text {crit }}$ wird im Folgenden der Minimalwert der Durchschlaglast oder der Verzweigungslast bezeichnet. 
Die ersten Untersuchungen an Kugelkappen unter einer Einzellast unter Berücksichtigung großer Verformungen wurden 1935 von Biezeno durchgeführt. In [9] untersuchte er Kugelkappen unter einer radialen Einzellast im Scheitel, die an den Rändern nur vertikal gelagert werden. Durch eine näherungsweise Lösung der nichtlinearen Differentialgleichungen ermittelte er die Durchschlaglast in Abhängigkeit der Schlankheit $\lambda$ für einen Bereich von $3,8<\lambda<8$, 6 . Biezeno ging dabei von einem rotationssymmetrischen Verformungszustand bei Erreichen der Durchschlaglast aus.

1956 untersuchte Chien in [10] die vertikal gelagerte Kugelkappe, die im Scheitel durch eine Ringlast belastet wurde. Er ging von einem rotationssymmetrischen Verformungszustand bis zum Durchschlagen der Schale aus. In einer Näherungslösung stellte er das nichtlineare Last-Verformungsverhalten in Abhängigkeit der Schlankheit der Kugelkappe im Bereich 3,65< $\lambda<5,4$ dar.

Ashwell betrachtete 1959 in einer analytischen Arbeit [11] die vertikal gelagerte Kugelkappe unter einer radialen Punktlast im Scheitel. Er unterteilte dabei die verformten Kugelkappen in zwei Bereiche: Den Bereich großer Verformungen in der Nähe der Lasteinleitung und den Randbereich, der nur kleine Verformungen erfährt. Ashwell formulierte eine Näherungslösung des nichtlinearen Problems, indem er jeweils getrennt für diese Bereiche die linearen Differentialgleichungen nach Reissner [1] verwendete. Ashwell nahm dabei rotationssymmetrische Verformungen bis zum Erreichen der Durchschlaglast an. In den analytischen Untersuchungen bestimmte er die kritische Last in Abhängigkeit vom Schlankheitsgrad $\lambda$ für den Bereich 3,75< $\lambda<7,5$. Zusätzlich erzielte er in einem Versuch mit einer Kugelkappe aus Aluminium und einem Schlankheitsgrad $\lambda$ von 9,7 sehr gute Übereinstimmungen mit seiner analytisch bestimmten LastVerformungsbeziehung, wobei er die gute Übereinstimmung des Versuchsergebnisses mit dem theoretischen Ergebnis als zufällig einstuft. Weder im Versuch, noch bei der analytischen Berechnung kam es zum Durchschlagen der Kugelkappe. In weiteren Versuchen mit Kugelkappen aus Aluminium $(4,7<\lambda<6,4) \mathrm{kam}$ es zum Durchschlagen der Kugelkappen unter einer punktförmigen Last.

In rund 70 Versuchen untersuchte Evan-Iwanowski 1962 in [12] das Tragverhalten von vertikal gelagerten und eingespannten Kugelkappen aus Aluminium, Stahl, Kunststoff (Vinylpolyethylen) und Kupfer. Die radiale Last wurde im Scheitel der Kugelkappe mit einem Metallstift eingeleitet. Bei Kugelkappen mit eingespannten Rändern konnte er kein Durschlagen im untersuchten Bereich von $5,3<\lambda<14,9$ beobachten. Die Last-Verformungskurven dieser Kugelkappen verlaufen monoton steigend. Vertikal gelagerte Kugelkappen mit einem Schlankheitsgrad $\lambda$ zwischen 3,7 und 10,2 schlugen dagegen durch. Bei Kugelkappen deren Schlankheitsgrad $\lambda$ größer als 10,2 ist, beobachtete Evan-Iwanowski asymmetrisches Beulen. Bei den untersuchten Kugelkappen trat zunächst ein dreiwelliges 
Beulmuster ein, das bei weiterer Laststeigerung in ein vierwelliges und schließlich fünfwelliges Beulmuster überging.

Mit Hilfe einer direkten Iterationmethode löste Archer 1965 in [13] numerisch die nichtlinearen Gleichungen nach Reissner [3]. Er bestimmte damit die LastVerformungskurven vertikal gelagerter Kugelkappen unter einer Punktlast. Der Schlankheitsgrad $\lambda$ der untersuchten Kugelkappen lag zwischen 3,5 und 20. Die Last-Verformungskurven verliefen monton steigend bis zu einem Punkt, an dem die Lösung nicht mehr konvergierte. Archer nahm an, dass an diesem Punkt die kritische Last erreicht ist und die Kugelkappen durchschlagen. Er ging dabei von einem axisymmetrischen Verformungszustand aus.

Mescall veröffentlichte 1965 in [14] die Last-Verformungskurven von Kugelkappen unter einer Punktlast. Dabei ermittelte er mit einem Iterationsverfahren die Lösung der nichtlinearen Gleichungen nach Reissner [3]. Bei den vertikal gelagerten Kugelkappen mit einem Schlankheitsgrad $\lambda$ zwischen 3,74 und 12 trat Durchschlagen auf. Waren die Ränder der Kugelkappen eingespannt, so trat Durchschlagen nur dann auf, wenn der Schlankeitsgrad $\lambda$ der Kugelkappen größer als 8 war. Die Art der Lasteinleitung und der Versagensmodus der vertikal gelagerten Kugelkappen ist für die analytischen Untersuchungen in Tabelle 2.1 und für die experimentellen Untersuchungen in Tabelle 2.2 zusammengefasst. Die kritischen Lasten der vertikal gelagerten Kugelkappen aus den theoretischen und experimentellen Untersuchungen [9] bis [13] wurden in Abbildung 2.3 über dem Schlankheitsgrad $\lambda$ aufgetragen. Bei Chiens Ergebnissen wurde der Grenzfall betrachtet, dass der Radius der Ringlast gleich Null ist.

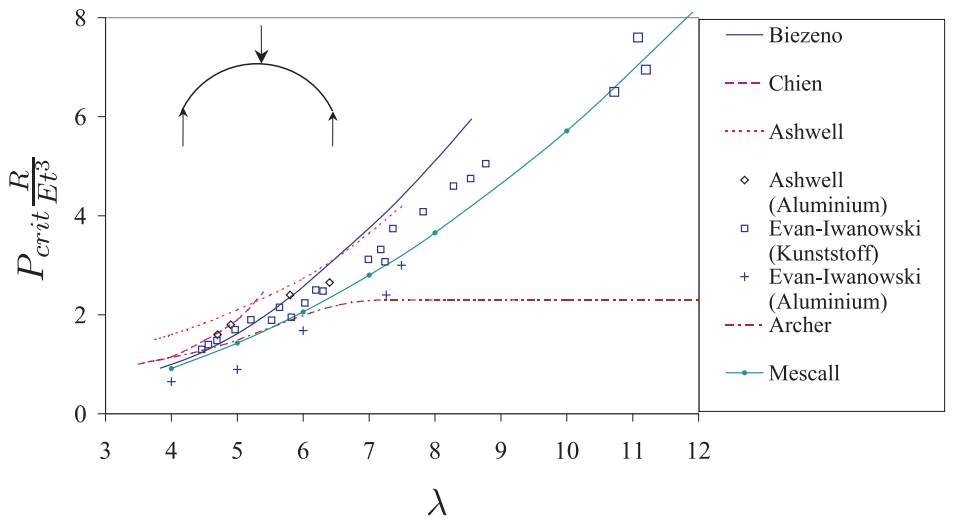

Abbildung 2.3: Kritische Lasten der vertikal gelagerten Kugelkappen aus theoretischen und experimentellen Untersuchungen 


\begin{tabular}{|l|l|l|l|}
\hline Autor & Last & Versagen & Parameterbereich \\
\hline \hline Biezeno [9] & Punktlast & Durchschlagen & $3,8 \leq \lambda \leq 8,6$ \\
\hline Chien [10] & Ringlast & Durchschlagen & $3,65 \leq \lambda \leq 5,4$ \\
\hline Ashwell [11] & Punktlast & Durchschlagen & $3,75 \leq \lambda \leq 7,5$ \\
\hline Archer [13] & Punktlast & Durchschlagen & $3,5 \leq \lambda \leq 20$ \\
\hline Mescall [14] & Punktlast & Spannungsproblem & $\lambda \leq 3,5$ \\
\hline Mescall [14] & Punktlast & Durchschlagen & $3,5<\lambda \leq 12$ \\
\hline
\end{tabular}

Tabelle 2.1: Analytische Untersuchungen an vertikal gelagerten Kugelkappen

\begin{tabular}{|l|l|l|l|}
\hline Author & Last & Versagen & Parameterbereich \\
\hline \hline Ashwell [11] & Flächenlast & Spannungsprob. & $\lambda=9,7$ \\
\hline Ashwell [11] & Punktlast & Durchschlagen & $4,7 \leq \lambda \leq 6,4$ \\
\hline $\begin{array}{l}\text { Evan } \\
\text {-Iwanowski [12] }\end{array}$ & Flächenlast & Spannungsprob. & $\lambda=3,7$ \\
\hline $\begin{array}{l}\text { Evan } \\
\text {-Iwanowski [12] }\end{array}$ & Flächenlast & Durchschlagen & $3,7<\lambda<10,2$ \\
\hline $\begin{array}{l}\text { Evan } \\
\text {-Iwanowski [12] }\end{array}$ & Flächenlast & asym. Beulen & $\lambda \geq 10,2$ \\
\hline
\end{tabular}

Tabelle 2.2: Experimentelle Untersuchungen an vertikal gelagerten Kugelkappen

Sowohl in den analytischen Untersuchungen von Biezeno [9], Chien [10], Ashwell [11], Archer [13] und Mescall [14] als auch in den experimentellen Untersuchungen von Ashwell [11] und Evan-Iwanowski [12] hat sich gezeigt, dass die Kugelkappen erst ab einem Schlankheitsgrad $\lambda$ von etwa 4 durchschlagen. Im Bereich $4<\lambda<6,5$ stimmen die Ergebnisse der theoretischen und experimentellen Untersuchungen von Biezeno, Chien, Ashwell, Evan-Iwanowski, Archer und Mescall gut überein. Die kritischen Lasten aus Evan-Iwanowskis Versuchen mit Kugelkappen aus Aluminium liegen weit unterhalb der kritischen Lasten, die in anderen Untersuchungen erzielt wurden. Die geringen kritischen Lasten sind auf plastische Verformungen in den Kugelkappen vor Erreichen der Durchschlaglast zurückzuführen. Für $\lambda>6,5$ weichen die Ergebnisse zum Teil erheblich voneinander ab. Die kritischen Lasten, die Archer in seinen analytischen Untersuchungen ermittelte, liegen deutlich unter denen von Biezeno, Ashwell, Evan-Iwanowski und Mescall. Weiterhin stimmen die Ergebnisse der theoretischen Untersuchungen von Mescall gut mit den experimentellen Ergebnisse der Kugelkappen aus Kuststoff von Evan-Iwanowski überein. Die kritischen Lasten, die Mescall ermittelte, liegen rund 15\% unterhalb der experimentell bestimmten Werten von Evan-Iwanowski. 
Penning und Thurston [15] führten 1965 analytische und experimentelle Untersuchungen an eingespannten Kugelkappen aus Aluminium durch. In den analytischen Untersuchungen gingen Penning und Thurston von Reissners Differentialgleichungen [3] und den nichtlinearen Dehnungs-Verschiebungsbeziehungen nach Sanders [16] aus. Neben rotationssymmetrischen Verformungen können dabei auch asymmetrische Verformungen berücksichtigt werden. In den Versuchen wurde die Last über ein kreisförmiges Rohr in den Scheitel der Kugelkappe eingeleitet. Der Durchmesser $d_{r}$ des Lasteinleitungsrohres im Verhältnis zum Grundkreisradius $L$ variierte zwischen 1/64 und 1/8. Die Last-Verformungskurven der analytischen Berechnungen waren stets monton steigend. Es trat bei keiner der berechneten Kugelkappen Durchschlagen oder asymmetrisches Beulen auf. Für Kugelkappen mit $\lambda<12$ trat auch bei den experimentellen Untersuchungen kein Durchschlagen oder asymmetrisches Beulen auf. Für Kugelkappen mit $\lambda \geq 12$ wurden Evan-Iwanowskis Beobachtungen bestätigt: In den Versuchen stellten sich anfangs kreisrunde Formänderungen ein, die bei weiterer Laststeigerung dreieckig, viereckig und schließlich fünfeckig wurden. Die im Versuch bis zum Auftreten von asymmetrischen Beulen gemessen Verformungen stimmten sehr gut mit den analytisch ermittelten Ergebnissen überein. Neben dem Einfluss des Schlankheitsgrades auf das Stabilitätsverhalten zeigte sich in diesen Untersuchungen, dass der Durchmesser der Lasteinleitungsfläche durchaus einen Einfluss auf die kritischen Lasten hat.

Penning veröffentlichte 1966 in [17] die Ergebnisse weiterer experimenteller Untersuchungen an eingespannten Kugelkappen aus Aluminium. Die Last wurde im Scheitel über einen Gummizylinder eingeleitet. Bei Kugelkappen, deren Schlankheitsgrad $\lambda$ kleiner als 8,68 war, kam es zu keinem Stabilitätsversagen. Der verformte Bereich blieb auch bei großen Lasten stets kreisförmig. Bei Kugelkappen mit Schlankheitsgraden $\lambda$ zwischen 8,68 und 16,6 traten drei-, vier- und fünfwellige Beulmuster auf.

Fitch [18] untersuchte 1968 eingespannte Kugelkappen unter radialer Einzellast im Scheitel. Ausgehend von Reissners Gleichungen ermittelte er numerisch die Eigenwerte, wobei der kleinste Eigenwert zur kritischen Last führte. Für $\lambda<7,8$ trat kein Durchschlagen auf. Für 7,8 $<\lambda<9,2$ hatten die LastVerformungskurven ein lokales Maximum, d.h. es kam zum Durchschlagen der Kugelkappen. Für $\lambda>9,2$ traten asymmetrische Beulmuster in den Kugelkappen auf, bevor der Durchschlagpunkt erreicht war.

Für die analytischen und experimentellen Untersuchungen ist in den Tabellen 2.3 und 2.4 die Art der Lasteinleitung, die Versagensart sowie der Parameterbereich, in dem dieses Versagen eintritt, zusammengefasst. In Abbildung 2.4 sind die entsprechenden kritischen Lasten aus [14] und [18], sowie ausgewählter Versuchskörper aus [15] und [17] über der Schlankheit $\lambda$ aufgetragen. Die Anzahl der Umfangsbeulwellen, die Fitch ermittelte, ist dabei mit $n$ bezeichnet. 


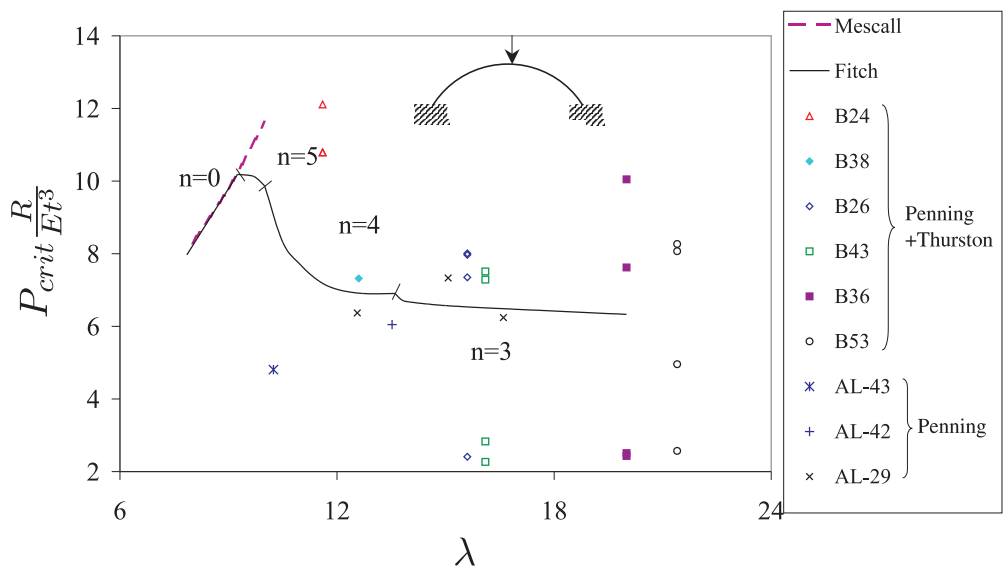

Abbildung 2.4: Kritische Lasten der eingespannten Kugelkappen aus analytischen und experimentellen Untersuchungen

\begin{tabular}{|l|l|l|l|}
\hline Autor & Last & Versagen & Parameterbereich \\
\hline \hline Mescall [14] & Punktlast & Spannungsproblem & $3,74 \leq \lambda<8$ \\
\hline Mescall [14] & Punktlast & Durchschlagen & $8 \leq \lambda \leq 12$ \\
\hline Penning [15] & Flächenlast & Spannungsproblem & $5 \leq \lambda \leq 21,4$ \\
\hline Fitch [18] & Punktlast & Spannungsproblem & $\lambda<7,8$ \\
\hline Fitch [18] & Punktlast & Durchschlagen & $7,8 \leq \lambda<9,2$ \\
\hline Fitch [18] & Punktlast & asym. Beulen & $\lambda \geq 9,2$ \\
\hline
\end{tabular}

Tabelle 2.3: Analytische Untersuchungen an eingespannten Kugelkappen

\begin{tabular}{|l|l|l|l|}
\hline Author & Last & Versagen & Parameterbereich \\
\hline \hline $\begin{array}{l}\text { Evan } \\
\text {-Iwanowski [12] }\end{array}$ & Flächenlast & $\begin{array}{l}\text { Spannungs- } \\
\text { problem }\end{array}$ & $5,3 \leq \lambda \leq 14,6$ \\
\hline Penning [15] & Flächenlast & asym. Beulen & $\lambda \geq 12$ \\
\hline Penning [17] & Flächenlast & asym. Beulen & $8,68 \leq \lambda \leq 16,6$ \\
\hline
\end{tabular}

Tabelle 2.4: Experimentelle Untersuchungen an eingespannten Kugelkappen

In allen Untersuchungen mit eingespannten Kugelkappen hat sich gezeigt, dass bei Kugelkappen mit kleinem Schlankheitsgrad kein Durchschlagen auftrat. In den Untersuchungen von Mescall und Fitch kam es bei Kugelkappen, deren Schlankheitsgrad $\lambda$ größer als 8 ist, zum Durchschlagen. Dabei stimmten die 
kritische Lasten dieser beiden Untersuchungen sehr gut überein. Der Vergleich der experimentellen Ergebnisse von Penning mit den analytischen Ergebnissen von Fitch ist nur bedingt möglich, da bei Penning und Thurston unterschiedliche Lasteinleitungsdurchmesser verwendet wurden und diese die kritische Last stark beeinflussen. Für gleiche $\lambda$-Werte ist die kritische Last bei kleinen Lastdurchmessern größer als bei großen Lastdurchmessern. Für kleine Lastdurchmesser liegen die kritischen Lasten rund 15\% bis 20\% oberhalb der Ergebnisse, die Fitch unter Annahme einer Punktlast angibt.

\subsection{Analytische und experimentelle Untersuchun- gen an Kreiszylinderschalen unter lokaler Be- lastung}

In diesem Abschnitt werden Kreiszylinderschalen betrachtet, wie sie in Abbildung 2.5 dargestellt sind. Diese Kreiszylinderschalen werden durch radial gerichtete Einzellasten $P$ beansprucht. Dabei greifen die Lasten an den Stutzen an, die einander gegenüberliegend angeordnet sind. Bei Kreiszylinderschalen gibt das Verhältnis des Radius $R$ zur Wanddicke $t$ Aufschluss über die Schlankheit der Struktur. Bei Kreiszylinderschalen mit lokaler Lasteinleitung ist zudem das Verhältnis des Lasteinleitungsdurchmessers $d_{r}$ zum Durchmesser $D$ der Kreiszylinderschale von Interesse. Der Abstand der Lasteinleitungsstelle zum Rand der Kreiszylinderschale wurde in den recherchierten Arbeiten nicht betrachtet.

Die ersten experimentellen Untersuchungen an Kreiszylinderschalen unter lokalen Einzellasten stammen von Roark [19] aus dem Jahr 1935. Er führte Spannungs- und Verschiebungsmessungen an Stahl- und Messingzylindern durch, deren $R / t$-Verhältnisse zwischen 36 und 90 lagen. Die Last $P$ wurde über abgerundete Stifte an zwei gegenüberliegenden Punkten an der Innenseite der Zylinder aufgebracht. Genaue Angaben zum Durchmesser des Lasteinleitungsstiftes sind in [19] nicht enthalten. Den zeichnerischen Darstellungen zufolge kann der Durchmesser $d_{r}$ aber annähernd als Punktlast angesehen werden. An den Enden der Kreiszylinder befanden sich vollkommen steife Platten.

1945 veröffentlichte Schoessow in [20] Ergebnisse von Versuchen an Stahlzylindern, an deren Stutzen radiale Zugkräfte aufgebracht wurden. Dabei wurden die Spannungen in der Nähe des Stutzenanschlusses gemessen. Die untersuchten Kreiszylinder hatten kleine $R / t$-Verhältnisse, die bei 13,5 und 20 lagen. Das Verhältnis des Stutzendurchmessers $d_{r}$ zum Durchmesser $D$ der Kreiszylinderschale lag bei 0,22. Die Ränder des Stahlzylinders waren auf steife Bleche geschweißt. 


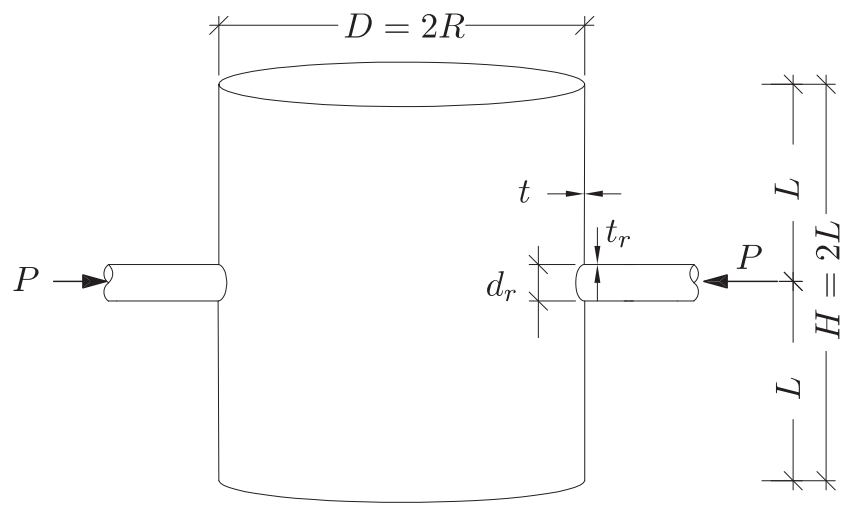

Abbildung 2.5: Kreiszylinderschale unter radialer Einzellast $P$

Die ersten theoretischen Untersuchungen auf diesem Gebiet wurden 1946 von Yuan [21] durchgeführt. Er ermittelte nach der geometrisch und physikalisch linearen Theorie der flachgekrümmten Schale nach Donnell [22] die radialen Verschiebungen zweier einander gegenüberliegender Punkte einer unendlich langen Kreiszylinderschale, die durch radiale, einander entgegengerichtete Punktlasten belastet wurden. Er stellte die radiale Verschiebung als Funktion der aufgebrachten Kraft $P$ und des $R / t$-Verhältnisses dar. In einer späteren Arbeit [23] vergleicht Yuan die Ergebnisse aus [21] mit Ergebnissen aus Berechnungen, denen die Differentialgleichungen nach Flügge [24] zugrunde liegen. Darüber hinaus ermittelt er die radialen Verschiebungen von endlich langen, gelenkig gelagerten Kreiszylinderschalen.

1955 untersuchte Bijlaard [25] Verformungen und Spannungen in Kreiszylinderschalen infolge lokaler Einzellasten. Die Last wurde dabei als schlaffes Lastbündel über eine kreisförmige oder quadratische Fläche eingeleitet. Die Ränder der Kreiszylinderschalen waren in radialer Richtung und in Umfangsrichtung gehalten. In seinen Untersuchungen, die auf der Donnellschen Schalentheorie [22] basieren, geht er von dünnwandigen Schalen mit mittleren $R / t$-Verhältnissen bis maximal 300 aus. Dabei werden große Verformungen nicht berücksichtigt. Diese sowie weitere Untersuchungen von Bijlaard auf diesem Gebiet ([26], [25] und [27]) resultieren im Welding research council bulletin (WRCB) 107 [8] und Anhang G des Britisch Standards BS5500. Dort sind die Spannungen infolge 
lokaler Einzellasten als Funktion der dimensionslosen Schalenparameter dargestellt. Dabei ist der Parameterbereich auf $R / t$-Verhältnisse bis 300 beschränkt. Die theoretischen Ergebnisse wurden 1960 von Cranch [28] durch den Vergleich mit experimentellen Untersuchungen an einem Kreiszylinder aus Stahl mit unterschiedlichen Stutzenausführungen verifiziert. Die dabei untersuchten Stahlzylinder hatten ein $R / t$-Verhältnis von 38 und $d_{r} / D$-Verhältnisse zwischen 0,135 und 0,0725. Da es sich bei den Versuchskörpern um geschlossene Behälter handelte, kann an den Ränder eine Einspannung angenommen werden.

Eine Erweiterung des WRCB 107 ist das WRCB 297 [29], das maßgeblich durch die Arbeiten von Steele ([30], [31], [32] und [33]) bestimmt wird. Neben einem erweiterten Parameterumfang, der auch Schalen mit $R / t$-Verhältnissen bis 1250 enthält und $d_{r} / D$-Verhältnisse zwischen 0,01 und 0,25 zulässt, sind auch unterschiedliche Verhältnisse der Dicke $t$ der Kreiszylinderschale zur Stutzendicke $t_{r}$ berücksichtigt. Große Verformungen werden dabei allerdings nicht berücksichtigt. Zudem können bei Einhaltung bestimmter Abmessungsverhältnisse die Spannungen in dem durch scheibenförmige Bleche verstärkten Stutzenanschlussbereich bestimmt werden. Steeles Arbeiten basieren auf den Gleichungen nach Flügge [34] und Sanders [35]. Die Ergebnisse von Steeles theoretischen Untersuchungen wurden in [31] mit Versuchsdaten der Chicago bridge and iron company (CBI) [36] $\left(R / t=524 ; 0,011 \leq d_{r} / D \leq 0,0278\right)$ und Gwaltney [37] $\left(25 \leq R / t \leq 50 ; 0,129 \leq d_{r} / D \leq 1\right)$ verglichen. Dabei wurden gute Übereinstimmungen zwischen experimentellen und analytischen Ergebnissen festgestellt. Für die von Morley in [38] hergeleitete Differentialgleichung werden in [39] Lösungen für unendlich lange dünnwandige Kreiszylinderschalen unter radialen Punktlasten präsentiert. Morley ermittelte mit seiner Methode die radialen Verformungen eines unendlich langen Kreiszylinders mit einem $R / t$-Verhältnis von 100 und stellt diese den Ergebnissen nach Yuan [21] gegenüber. Beide gehen dabei von der geometrisch und physikalisch linearen Theorie aus.

Ausgehend von Donnells Differentialgleichung führte Kupfer 1990 in [40] umfangreiche Serienberechnungen zur Ermittlung der Spannungen in Kreiszylinderschalen unter Stutzenlasten durch. Die Berechungsdiagramme berücksichtigen unterschiedliche Stutzensteifigkeiten und ermöglichen die Ermittlung der maximalen Vergleichsspannung in der Kreiszylinderschale und im Stutzen für kleine und mittlere $R / t$-Verhältnisse zwischen 5 und 500. Die Duchmesserverhältnisse $d_{r} / D$ sind dabei auf einen Bereich zwischen 0,01 und 0,3 beschränkt.

Die auf T-Knotenverbindungen von dickwandigen Rohren ausgelegten Formeln zur Spannungsermittlung nach Wordsworth werden in [41] auch auf dünnwandige Kreiszylinderschalen angewandt. Bedingt durch den ursprünglichen Anwendungsbereich dieser Formeln ist der Parameterbeich auf $R / t$-Verhältnisse von maximal 50 beschränkt. Große Verformungen werden dabei nicht berïcksichtigt. 
Dekker führte in [42] einen Vergleich der ermittelten Spannungen nach WRCB 107, Anhang G des BS 5500 und den Formeln nach Wordsworth durch. Dieser Vergleich zeigte erhebliche Abweichungen für große Durchmesserverhältnisse auf. Aufgrund dieser Abweichungen wird die Anwendung der Formeln nach Wordsworth nur für kleine $R / t$-Verhältnisse empfohlen.

Zur Spannungsermittlung in kreiszylindrischen Behältern unter Einwirkung von Innendruck und Rohrleitungslasten liegen Reihenuntersuchungen mit der FEMethode in den Arbeiten von Rudolph [43] und Joost [44] vor. In einer darauf aufbauenden Arbeit von Rauth [45] wurden vollparametrisierte FE-Modelle erstellt, die dem Anwender die Spannungsermittlung im Stutzenanschlussbereich ermöglichen. Diese Untersuchungen berücksichtigen die plastischen Werkstoffeigenschaften. Dabei wird von geometrisch linearem Verhalten ausgegangen, wodurch der Anwendungsbereich auf $R / t$-Verhältnisse bis 200 beschränkt ist.

Die in diesem Abschnitt beschriebenen Untersuchungen sind in Tabelle 2.5 unter Angabe des $R / t$-Verhältnisses und des Durchmesserverhältnisses $d_{r} / D$ zusammengefasst und in Abbildung 2.6 in Abhängigkeit vom $R / t$ und $d_{r} / D$-Verhältnis aufgetragen. Da das WRCB 107 aus Biljaards Arbeiten hervorgeht und weitgehend identisch mit BS 5500 ist, wurde in Abbildung 2.6 nur der Parameterbereich des WRCB 107 aufgeführt. Gleiches gilt für Steeles Ergebnisse, die in WRCB 297 einfließen und folglich den gleichen Parameterbereich umfassen.

\begin{tabular}{|l|l|l|l|}
\hline Autor & Untersuchungsart & $R / t$ & $d_{r} / D$ \\
\hline \hline Roark [19] & experimentell & $45-90$ & $\approx 0$ \\
\hline Schoessow [20] & experimentell & $13,5-20$ & 0,22 \\
\hline Yuan [21] & analytisch & $0-1750$ & 0 \\
\hline Bijlaard [25]-[27] & analytisch & $15-300$ & $0,01-0,25$ \\
\hline WRCB 107 [8] & analytisch & $5-300$ & $0,01-0,57$ \\
\hline Cranch [28] & experimentell & 38 & $0,0725-0,135$ \\
\hline Steele [30]-[33] & analytisch & $25-1250$ & $0,01-0,25$ \\
\hline BS 5500 & analytisch & $10-300$ & $0,02-0,5$ \\
\hline WRCB 297 [29] & analytisch & $50-1250$ & $0,01-0,5$ \\
\hline CBI [36] & experimentell & 524 & $0,011-0,0278$ \\
\hline Gwaltney [37] & experimentell & $25-50$ & $0,129-1$ \\
\hline Kupfer [40] & analytisch & $5-500$ & $00,1-0,3$ \\
\hline Wordsworth [41] & analytisch & 50 & $0,05-0,55$ \\
\hline Rauth [45] & FEM & $20-200$ & $0,1-0,8$ \\
\hline
\end{tabular}

Tabelle 2.5: Experimentelle und analytische Untersuchungen an Kreiszylinderschalen 


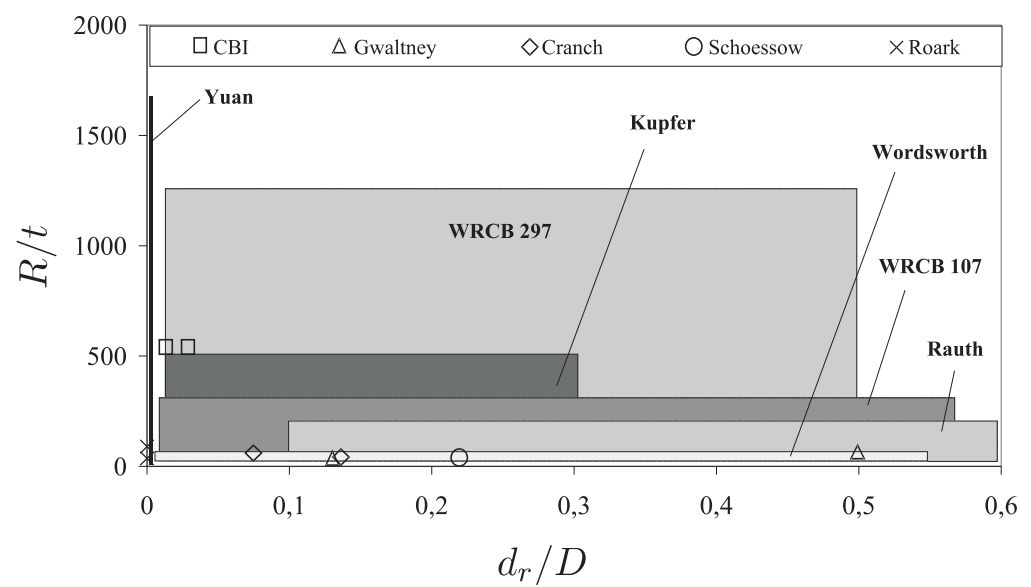

Abbildung 2.6: Zusammenstellung der Untersuchungen an Kreiszylinderschalen unter radialen Einzellasten

Außer Einzellasten, die durch Stutzen und Anbauteile hervorgerufen werden, tritt bei exzentrisch befüllten Kreiszylinderschalen wie Silos eine Beanspruchung der Schalenwand durch Einzellasten auf. Diese Teilflächenlasten sind in DIN 1055 Teil 6 geregelt. Die Spannungsverteilung in diesem Bereich, die zum Versagen führen kann, wurde in [46] für radiale Lasten grundlegend untersucht. In [47] wurde dieses Problem unter Berücksichtigung von geometrischer und materieller Nichlinearität betrachtet und die Auswirkungen auf das Stabilitätsverhalten der Schale untersucht. Die lokale, nach außen gerichtete radiale Last greift dabei an einer rechteckigen Fläche an. Im Vergleich zur Fläche, die ein Stutzenanschluss einnimmt, sind diese Teilflächen jedoch so groß, dass ein Vergleich mit Stutzenlasten nicht sinnvoll ist.

Eine lokale Momentenbelastung tritt bei Silos auf, die mit Konsolen auf Stützen gelagert sind. Dieses Problem wurde eingehend in [48], [49] und [50] untersucht, wobei jedoch das Kontaktproblem des dort erforderlichen Verstärkungsbleches außer acht blieb. Unter Berücksichtigung geometrischer und materieller Nichlinearität sowie geometrischer Imperfektionen wurde in Parameterstudien der Einfluss der Konsolenabmessungen auf das Last-Verformungsverhalten der Struktur untersucht. Das globale Verformungsverhalten gleicht dem Verhalten einer Kreiszylinderschale unter Stutzen- und Rohrleitungslasten. 


\subsection{Behandlung des Problems in Normen und Re- gelwerken}

Der durch den Stutzenanschluss entstehende Mantelausschnitt ist in den meisten Vorschriften nur für die Belastung durch Innendruck des kreiszylindrischen Behälters geregelt und wird durch Anwendung des Flächenersatzverfahrens berücksichtigt. Dabei wird die ausgeschnittene Fläche durch eine benachbarte Fläche ersetzt. Als Ersatzfläche können die nicht ausgenutzten Mantelflächen sowie Teile des Stutzens selbst herangezogen werden. Wenn die von Stutzen und dem nicht ausgenutzten Teil des Mantelbleches zur Verfügung stehende Ersatzfläche nicht ausreicht, kann eine Verstärkung des Mantelbleches im Stutzenbereich erforderlich sein. Mit dem Flächenersatzverfahren werden nur Umfangszugpannungen aus Innendruck berücksichtigt. Spannungen infolge lokaler Lasten am Stutzenanschluss werden durch das Flächenersatzverfahren nicht erfasst.

In DIN 4119-2:1980-02, „Oberirdische zylindrische Flachboden-Tankbauwerke aus metallischen Werkstoffen“ und in DIN EN 14015:2005-02, „Auslegung und Herstellung standortgefertigter, oberirdischer, stehender, zylindrischer, geschweißter Flachboden-Stahltanks für die Lagerung von Flüssigkeiten bei Umgebungstemperatur und höheren Temperaturen“, die DIN 4119-2 teilweise ersetzt und weitgehend aus API 650 und BS 2654 hervorgeht, entfällt bei Anwendung des Flächenersatzverfahrens der Nachweis der Spannungen aus Innendruck.

Die Richtlinie für die Herstellung von Flachbodentanks mit besonderen Anforderungen, Vereinbarung 2002/1 zwischen FDBR und VdTÜV (2002) verweist bei der Bemessung der Ausschnitte auf AD 2000-Merkblatt B9:,,Ausschnitte in Zylindern, Kegeln und Kugeln“ (2000-10). Für die Belastung durch Innendruck werden Verstärkungsmaßnahmen im Sinne des Flächenersatzverfahrens gefordert. Zur Erfassung der lokalen Spannungen hervorgerufen durch Stutzenlasten wird auf AD 2000-Merkblatt S3/9: „Allgemeiner Standsicherheitsnachweis für Druckbehälter: Behälter mit Stutzen unter Zusatzbelastung“ (2001-09) verwiesen, das wiederum zu Ermittlung der Spannungen infolge Stutzenlasten auf WRCB 107 [8] oder WRCB 297 [29] verweist.

\subsection{Zusammenfassung}

Die in den Tabellen 2.1, 2.2, 2.3 und 2.4 aufgeführten Untersuchungen über lokal beanspruchte Kugelkappen unter Berücksichtigung des nichtlinearen LastVerformungsverhaltens haben gezeigt, dass das Tragverhalten dieser Strukturen vom Schlankheitsgrad $\lambda$ und von den Randbedingungen abhängt. In einem 
Großteil der analytischen Untersuchungen wurde die Last über einen singulären Punkt eingeleitet. Unterschiedliche Lasteinleitungsdurchmesser wurden nur in [15] und [17] explizit betrachet. Die Untersuchungen zur Spannungsermittlung in der durch lokale Lasten beanspruchten Kreiszylinderschale nach Tabelle 2.5 sind, wie Abbildung 2.6 untermauert, auf Kreiszylinderschalen mit kleinen und mittleren $R / t$-Verhältnissen beschränkt. Alle aufgeführten Untersuchungen berücksichtigen weder geometrische noch materielle Nichtlinearität. Da viele dieser Untersuchungen aus dem Umfeld des Anlagenbaus stammen, werden auch sehr große $d_{r} / D$-Verhältnisse bis etwa 0,6 erfasst, während diese Werte bei großen Tankbauwerken in der Regel im Bereich zwischen 0,002 und 0,1 liegen. 


\section{Berechnungen mit der Finiten Elemente Methode - Vorüberlegungen und Grundlagen}

\subsection{Allgemeines}

Die numerischen Untersuchungen wurden mit dem FE-Programmsystem ANSYS 7.1 [51] durchgeführt. Bei Untersuchungen mit der Methode der Finiten Elemente hängt die Genauigkeit der Ergebnisse stark von der Wahl geeigneter Elemente, der Feinheit des Netzes sowie der Eignung des Lastverfolgungsalgorithmus ab. Darüber hinaus müssen die Randbedingungen und Werkstoffeigenschaften der Strukturen und die Lasteinleitung in geeigneter Weise modelliert werden.

\subsection{Verwendete Elemente}

\subsubsection{Schalenelemente}

Die Kugelkappen und Kreiszylinderschalen werden als dreidimensionale Modelle abgebildet. Für die Diskretisierung dieser dünnwandige Strukturen werden Schalenelemente des Typs SHELL181 [51] verwendet. Dabei handelt es sich um isoparametrische Schalenelemente mit vier Knoten, die jeweils über drei translatorische Freiheitsgrade (in $x$-, $y$ - und $z$-Richtung) und drei Rotationsfreiheitsgrade (um die $x$-, $y$ - und $z$-Achse) verfügen. Das verwendete Schalenelement bietet die Möglichkeit, in den Berechnungen plastisches Werkstoffverhalten zu berücksichtigen.

\subsubsection{Kontaktelemente}

Bei der Modellierung zweier übereinanderliegender Elementschichten muss einerseits verhindert werden, dass die Elemente einander gegenseitig durchdringen, andererseits müssen Kräfte normal und tangential zu den Elementen übertragen 
3 Berechnungen mit der Finiten Elemente Methode - Vorüberlegungen und Grundlagen

werden. Diese sogenannte Kontaktfläche kann mit Kontaktelementen modelliert werden, die sich in der Fuge zwischen beiden Elementschichten befinden. In ANSYS 7.1 besteht die Möglichkeit, mit einem Kontaktelementpaar bestehend aus dem Kontaktelement CONTACT174 und dem Zielelement TARGET170 Druckund Schubkräfte zwischen den Elementen zu übertragen. Das Elementenpaar wird dabei auf die bereits bestehenden Elemente gelegt. Sobald Kontakt zwischen Kontakt- und Zielelement festgestellt wird, werden Druck- und Schubkräfte übertragen. Die Steifigkeit des Kontaktelements bezieht sich dabei auf die Steifigkeit des darunterliegenden Schalenelements und kann über einen Faktor skaliert werden. Die Übertragung von Kräften tangential zur Kontaktfläche wird über den Reibkoeffizient $\mu$ berücksichtigt.

\subsection{Das FE-Modell der Kugelkappe}

\subsubsection{Lasteinleitung}

Bei der Art der lokalen Lasteinleitung wird im Rahmen dieser Arbeit zwischen zwei Varianten unterschieden. Dies ist zum einen die Kugelkappe nach Abbildung 3.1(a), an deren Scheitel die Last $P$ über einen kreisförmigen Querschnitt, der unabhängig von der Kugelkappe ist, gleichförmig verteilt eingeleitet wird. Im FE-Modell wird diese Art der Lasteinleitung berücksichtigt, indem die Last direkt auf die Kugelkappe aufgebracht wird. Bei der anderen Variante nach Abbildung 3.1(b) ist der Stutzen fest mit der Kugelkappe verbunden. Da sich die Beschaffenheit des Stutzens auf das Tragverhalten der Kugelkappen auswirken kann, wird dieser mit Schalenelementen modelliert.

\subsection{2 $\quad$ FE-Modell der Kugelkappe mit Lasteinleitung über eine Fläche}

Bei einer Kugelkappe, wie sie in Abbildung 3.1(a) dargestellt ist, mit dem Radius $R$, der Wanddicke $t$ und dem Grundkreisradius $L$ wird im FE-Modell die Last über eine kreisförmige Fläche am Scheitel eingeleitet. Dabei hat die Lasteinleitungsfläche einen Durchmesser $d_{r}$. Die Wanddicke im Lasteileitungsbereich wird mit $t_{p}$ bezeichnet. Das Modell dieser Kugelkappe ist in Abbildung 3.2 dargestellt.

Die Rotationssymmetrie der Kugelkappe unter Einwirkung einer radialen, am Scheitel der Kugelkappe angreifenden Einzellast $P$ wird bei der Modellierung ausgenutzt, indem nur ein Viertel der Kugelkappe abgebildet wird. Die Ränder an den Symmetrieebenen werden entsprechend den Symmetrierandbedingungen 
(a)

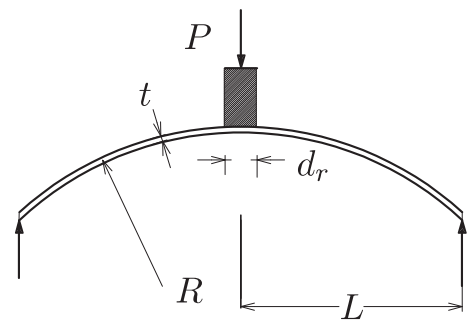

(b)

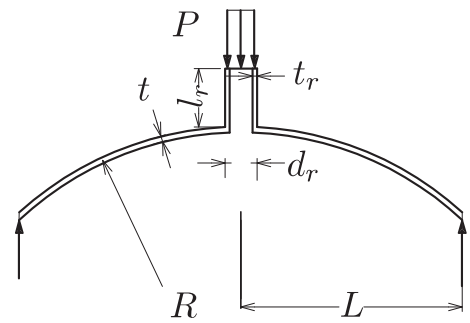

Abbildung 3.1: Kugelkappen unter radialer Einzelast mit (a) direkter Lasteinleitung und (b) Lasteinleitung über einen Stutzen

nach Abbildung 3.2 gelagert. Da in der Nähe der Lasteinleitung ein sehr großer Spannungsgradient erwartet wird, wird das Netz im Radius von $d_{n} / 2=10 \sqrt{R t}$ um die Lasteinleitungsfläche sehr fein ausgeführt. Außerhalb dieses Bereichs ist ein hoher Feinheitsgrad nicht erforderlich. Der untere Rand der Kugelkappe wird nur vertikal aufgelagert (Modell KFV) oder ist eingespannt (Modell KFE). In Abbildung 3.3 ist das FE-Netz einer Kugelkappe abgebildet.

\subsubsection{FE-Modell der Kugelkappe mit Lasteinleitung über einen Stutzen}

Die Kugelkappe nach Abbildung 3.1(b) hat am Scheitel einen Stutzen mit dem Durchmesser $d_{r}$, der Wanddicke $t_{r}$ und der Länge $l_{r}$, der fest mit der Kugelkappe verbunden ist. Die Last wird als Streckenlast am freien Ende des Stutzens aufgebracht.

Das in Abbildung 3.4 dargestellte FE-Modell der Kugelkappe entspricht - mit Ausnahme des Stutzens - dem in Abschnitt 3.3.2 beschriebenen Modell. Das FENetz der Kugelkappe ist in Abbildung 3.5 dargestellt. Es werden Modelle der Kugelkappe mit vertikal aufgelagertem Rand (Modell KSV) und eingespanntem Rand (Modell KSE) erstellt. 
3 Berechnungen mit der Finiten Elemente Methode - Vorüberlegungen und Grundlagen
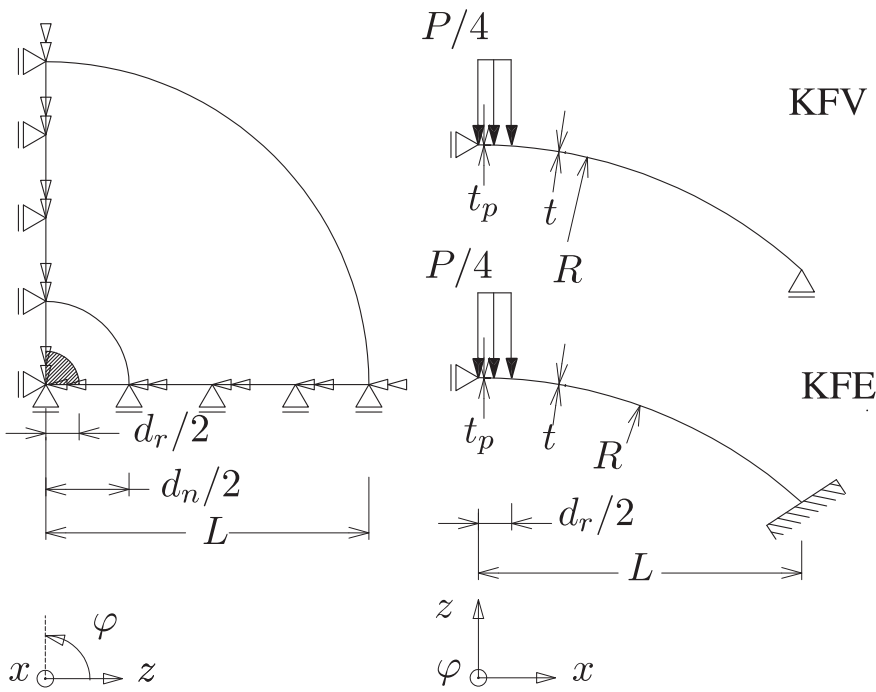

Abbildung 3.2: FE-Modell einer Kugelkappe mit Lasteinleitung über eine Fläche mit dem Durchmesser $d_{r}$

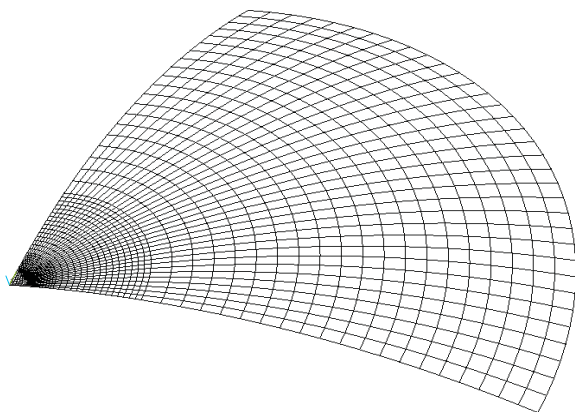

Abbildung 3.3: FE-Netz der Kugelkappe mit einer Lasteinleitungsfläche am Scheitel 
3 Berechnungen mit der Finiten Elemente Methode - Vorüberlegungen und Grundlagen

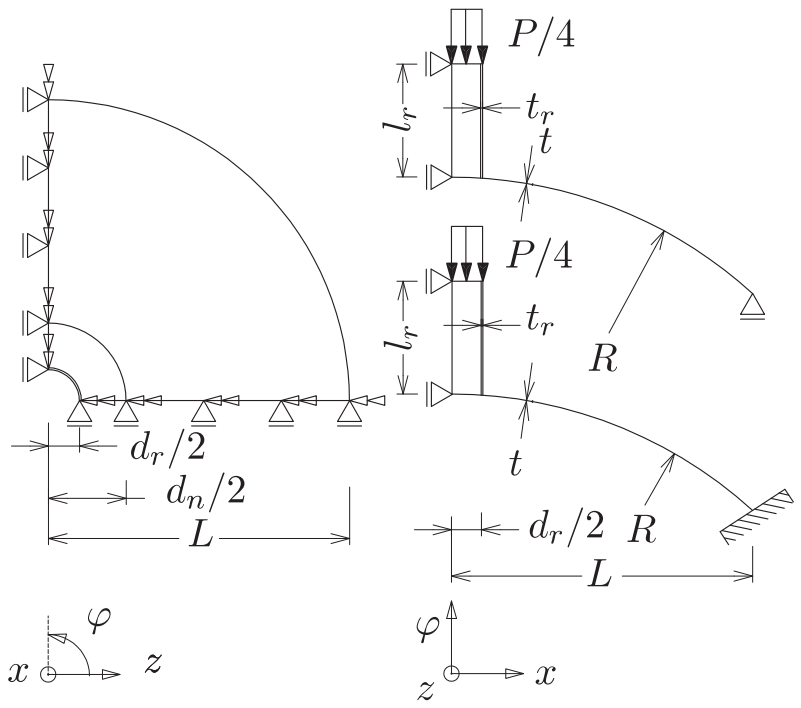

Abbildung 3.4: FE-Modell der Kugelkappe mit Lasteinleitung über einen Stutzen mit dem Durchmesser $d_{r}$

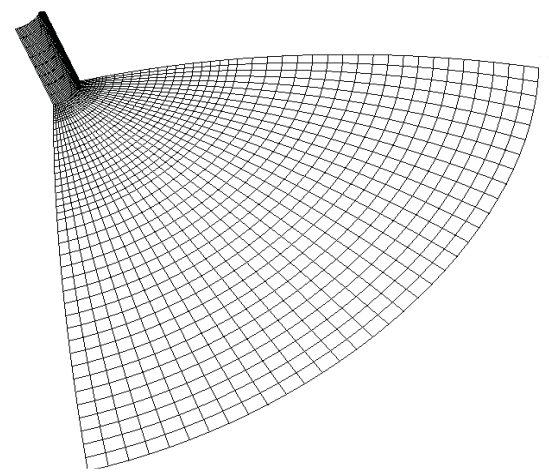

Abbildung 3.5: FE-Netz der Kugelkappe mit einem Stutzen 


\subsection{FE-Modell der Kreiszylinderschale}

Im Gegensatz zu den Kugelkappen werden bei den Kreiszylinderschalen nur solche Fälle numerisch untersucht, in denen der Stutzen fest mit der Schalenwand verbunden ist. Die in Abbildung 3.6 dargestellte Kreiszylinderschale mit dem Radius $R$ und der Wanddicke $t$ hat einen Stutzen mit dem Durchmessers $d_{r}$, der Wanddicke $t_{r}$ und der Länge $l_{r}$. Der Stutzen hat dabei einen Abstand $L$ zum oberen und unteren Rand der Kreiszylinderschale.

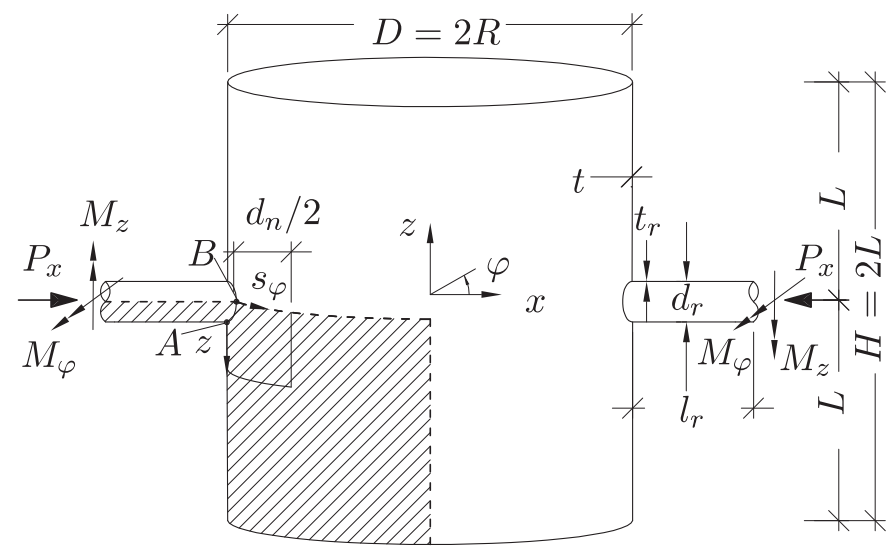

Abbildung 3.6: FE-Modell der Kreiszylinderschale unter lokaler Belastung

Es werden die Lastfälle

- radiale Einzellast $P_{x}$,

- Moment in Umfangsrichtung $M_{\varphi}$ und

- Moment in Meridianrichtung $M_{z}$

untersucht. Das Moment in Umfangsrichtung $M_{\varphi}$ erzeugt dabei Biegespannungen in Meridianrichtung und entspricht daher der Bezeichnung $m_{x}$ in DIN V ENV 1993-1-6:2005-05. Das Moment in Meridianrichtung $M_{z}$ entspricht folglich dem Moment $m_{y}$, das Biegespannungen in Umfangsrichtung erzeugt. 
3 Berechnungen mit der Finiten Elemente Methode - Vorüberlegungen und Grundlagen

Die radiale Einzellast $P_{x}$ wird am freien Ende des Stutzens als Streckenlast aufgebracht. Das freie Ende des Stutzens ist dabei wölbbehindert. Bei Einwirkung eines Moments wird das Moment in ein Kräftepaar aufgeteilt und am freien Ende des Stutzens aufgebracht.

Für den oberen und unteren Rand der Kreiszylinderschale werden die Randbedingungen mit den Kurzbezeichnung nach DIN 18800-4:1990-11 betrachet. Diese sind

- ein eingespannter Rand mit $u=0, v=0$ und $\operatorname{rot}_{\varphi}=0$ (RB1),

- ein gelenkiger Rand $u=0, v=0$ (RB2) und

- ein freier Rand (RB3).

Die Symmetrierandbedingungen sind in Abbildung 3.7 dargestellt.

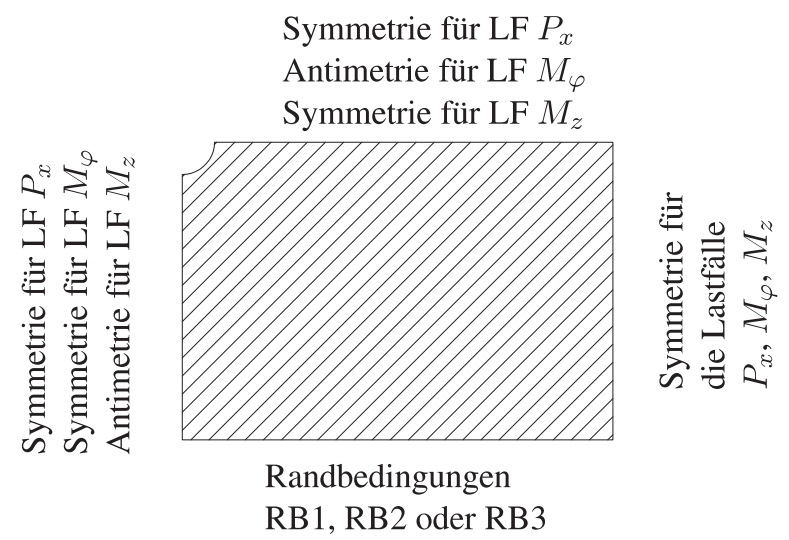

\section{Abbildung 3.7: FE-Modell der Kreiszylinderschale unter lokaler Belastung}

Das auch im Schrifttum verwendete Modell gemäß Abbildung 3.6 mit zwei einander gegenüberliegenden Stutzen wurde deshalb gewählt, weil sich bei diesem keine globale Lastabtragung in Längsrichtung der Kreiszylinderschale ergibt. Damit auch in Umfangsrichtung keine bemerkenswerte gegenseitige Beeinflussung der Beanspruchung durch die aufgebrachten Lasten erfolgt, muss sichergestellt sein, dass diese in Umfangsrichtung rasch abklingen. Die Symmetrie der Struktur wird ausgenutzt, indem im FE-Modell ein Viertel des Umfangs und die halbe Höhe des Zylinders abgebildet wird. Die Symmetrierandbedingungen ergeben 
3 Berechnungen mit der Finiten Elemente Methode - Vorüberlegungen und Grundlagen

sich aus dem entsprechenden Lastfall und sind ebenfalls in Abbildung 3.6 aufgeführt. Das Finite Elemente Modell der Kreiszylinderschale ist in Abbildung 3.8 dargestellt. Die feine Vernetzung, die aufgrund der großen Spannungsgradienten im Lasteinleitungsbereich erforderlich ist, muss nicht für die gesamte Kreiszylinderschale eingehalten werden. Der sehr fein vernetzte Bereich beschränkt sich auf einen Bereich von $d_{n} / 2$ um die Stutzenachse. Der Radius dieses Bereichs wird mit $10 \sqrt{R t}$ angenommen.

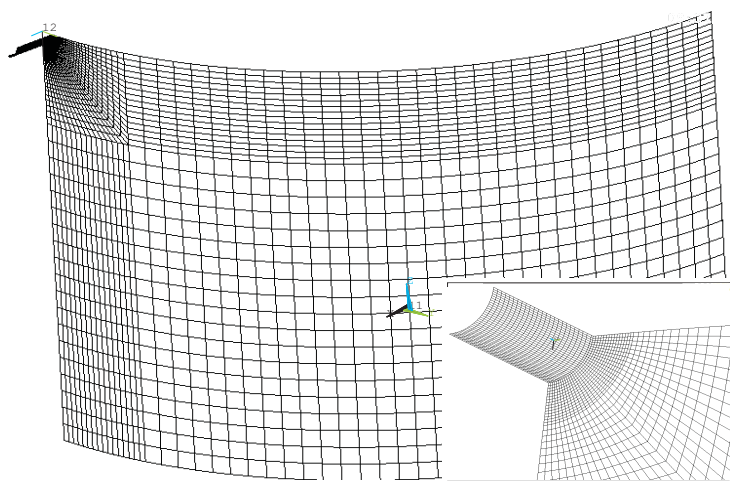

Abbildung 3.8: Das FE-Modell der Kreiszylinderschale mit Stutzen

\subsection{Werkstoffgesetz}

In den numerischen Untersuchungen werden Strukturen aus unlegierten Baustählen nach DIN EN 10025 betrachtet. Vereinfachend wird dabei ein linear-elastisch ideal-plastisches Werkstoffgesetz mit einer geringen Verfestigung nach Abbildung 3.9 angenommen. Der Werkstoff hat einen E-Modul $E$ von $210000 \mathrm{~N} / \mathrm{mm}^{2}$ und eine Querdehnzahl von $\nu=0,3$. Der Verfestigungsmodul $E_{v}$ beträgt ein Hundertstel des E-Moduls $E$. Der E-Modul des Stutzen wird mit $E_{r}$ bezeichnet. 


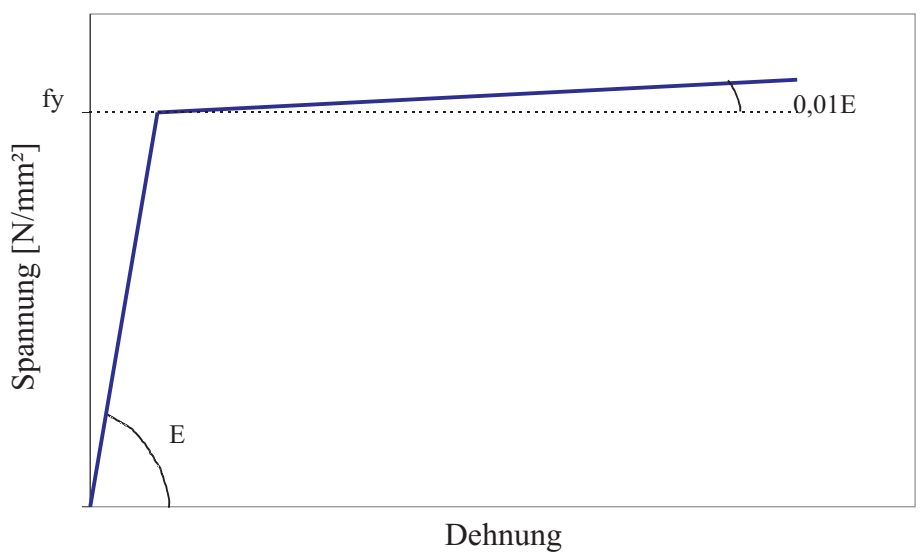

Abbildung 3.9: Spannungs-Dehnungsdiagramm des verwendeten Werkstoffs

\subsection{Lastverfolgungsalgorithmus}

Bei nichtlinearen Berechnungen treten nichtlineare Gleichungssysteme auf, die im allgemeinen nicht direkt lösbar sind, sondern mit Hilfe eines Iterationsverfahrens gelöst werden müssen. Die gebräuchlichste Iterationsmethode ist dabei das Newton-Raphson-Verfahren, das auch in ANSYS 7.1 implementiert ist [51]. Mit Hilfe des Newton-Raphson-Verfahrens können Gleichgewichtszustände auf einem Last-Verformungspfad iterativ bestimmt werden. In einer lastgesteuerten Berechnung wird der Verschiebungszustand zu einer vorgegebenen Last gesucht. Dabei kann Konvergenz allerdings nur für positive Steifigkeiten erzielt werden, was bedeutet, dass Gleichgewichtszustände im Nachbeulbereich nicht ermittelt werden können. In einem verschiebungsgesteuerten Verfahren ist es möglich, den Last-Verformungspfad über diesen singulären Punkt hinweg zu verfolgen. Allerdings ist eine verschiebungsgesteuerte Berechnung nur dann möglich, wenn der Lastvektor dem Verschiebungsvektor entspricht. Bei einer lastgesteuerten Berechnung kann zum Durchfahren des Last-Verformungspfades bis in den Nachbeulbereich hingegen ein von Riks [52], [53] entwickelter Algorithmus eingesetzt werden. Von Crisfield [54] wurde dieses Verfahren weiterentwickelt und ist als sogenanntes Bogenlängenverfahren in ANSYS 7.1 implementiert. Mit dem Bogenlängenverfahren gelangt man auch über lokale Maxima des LastVerformungspfades hinweg in den Nachbeulbereich. Wenn allerdings Verzwei- 
3 Berechnungen mit der Finiten Elemente Methode - Vorüberlegungen und Grundlagen

gungspunkte unterhalb des lokalen Maximums vorhanden sind, so werden diese vom Bogenlängenverfahren überfahren. Diese Verzweigungspunkte können in einer begleitenden Eigenwertanalyse aufgespürt werden.

Aufgrund der geschilderten besonderen Eignung für die Problemstellung dieser Arbeit wurde das Bogenlängenverfahren für die FEM-Untersuchungen eingesetzt. 


\section{Das Tragverhalten der lokal belasteten Kugelkappe}

\subsection{Hintergrund}

Das Tragverhalten einer lokal beanspruchten Kugelkappe, wie sie in Abbildung 4.1 dargestellt ist, wird in diesem Kapitel mit Hilfe von Analogiebetrachtungen und Vergleichsberechnungen mit lokal beanspruchten Strukturen untersucht. Dabei wird analysiert, welche Parameter einen Einfluss auf das Tragverhalten haben und welche Rolle dabei die Randbedingungen und die Art der Lasteinleitung spielen.

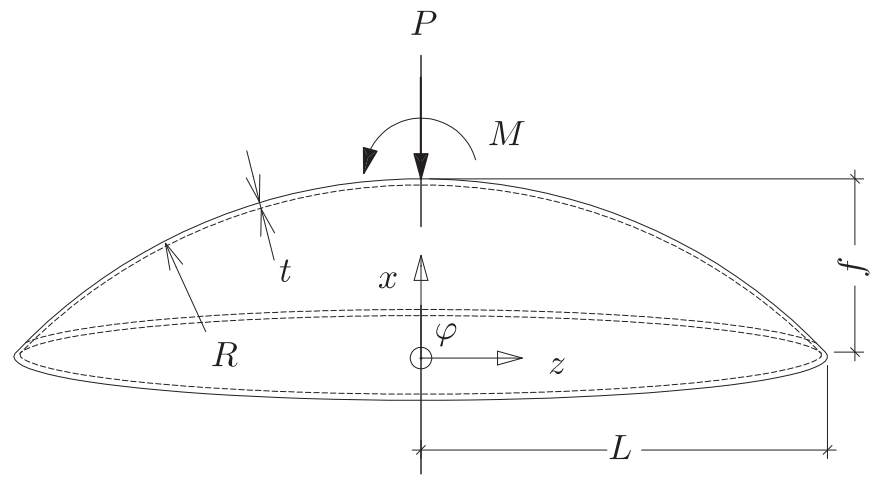

Abbildung 4.1: Lokal beanspruchte Kugelkappe

\subsection{Analogie von Kugelkappe und elastisch gebette- ter Kreisplatte}

Das geometrisch und werkstofflich lineare Tragverhalten der Kugelkappe, an deren Scheitel eine radial gerichtet Einzellast $P$ angreift (vgl. Abbildung 4.1), ent- 
spricht dem einer elastisch gebetteten Kreisplatte unter einer in Feldmitte angreifenden Last $P$ nach Abbildung 4.2. Betrachtet man die Differentialgleichungen der Kugelkappe unter einer im Scheitel angreifenden Einzellast $P$ nach Reissner [1]

$$
\underbrace{\Delta \Delta u \cdot \frac{E t^{3}}{12\left(1-\nu^{2}\right)}}_{\text {Biegeanteil }}+\underbrace{\frac{E t}{R^{2}} u}_{\text {Membrananteil }}=P
$$

mit dem Operator

$$
\Delta=\frac{d^{2}}{d z^{2}}+\frac{1}{L} \cdot \frac{d}{d z}
$$

und vergleicht diese mit der Differentialgleichung der elastisch gebetteten Kreisplatte

$$
\Delta \Delta u_{P, k} \cdot \frac{E t^{3}}{12\left(1-\nu^{2}\right)}+k u_{P, k}=P
$$

so zeigt sich, dass der erste Term der Differentialgleichung der Kugelkappe und der ersten Term der Differentialgleichung der elastisch gebetteten Kreisplatte identisch sind. In der Differentialgleichung der Kugelkappe beschreibt der erste Term den Biegeanteil der Lastabtragung, der zweite Term die Membrantragwirkung der Struktur. Wird der zweite Term aus Gleichung 4.3, der die elastische Bettung der Kreisplatte beschreibt, dem zweiten Term auf Gleichung 4.1 gleichgesetzt, so kann die Steifigkeit $k$ der elastischen Bettung der Kreisplatte mit

$$
k=\frac{E t}{R^{2}}
$$

der Membransteifigkeit der Kugelkappe gleichgesetzt werden.

Das Tragverhalten der Kugelkappe kann durch eine Analogiebetrachtung mit einer elastisch gebetteten Kreisplatte mit der Steifigkeit $k$ der elastischen Bettung, die von $E, t$ und $R$ abhängt, anschaulich erläutert werden. Dazu wurde eine Kreisplatte mit einem Radius $L$ von $10000 \mathrm{~mm}$, einer Dicke $t$ von $10 \mathrm{~mm}$, einem E-Modul von $210000 \mathrm{~N} / \mathrm{mm}^{2}$ sowie einer Querdehnungszahl $\nu=0,3$ betrachtet. Die elastische Bettung $k$ der Kreisplatte wurde in Abhängigkeit von $R$ der äquivalenten Kugelkappe variiert. Mit Hilfe eines Programms zur Berechnung rotationssymmetrischer Schalen (ROTASS [55]) wurden die Durchbiegungen $u_{P, k}$ der elastisch gebetteten Kreisplatte unter der Last $P$ für unterschiedliche $k$ ermittelt. Die Durchbiegung $u_{P, k}$ wurde mit der Durchbiegung $u_{P}$ der ungebetteten 


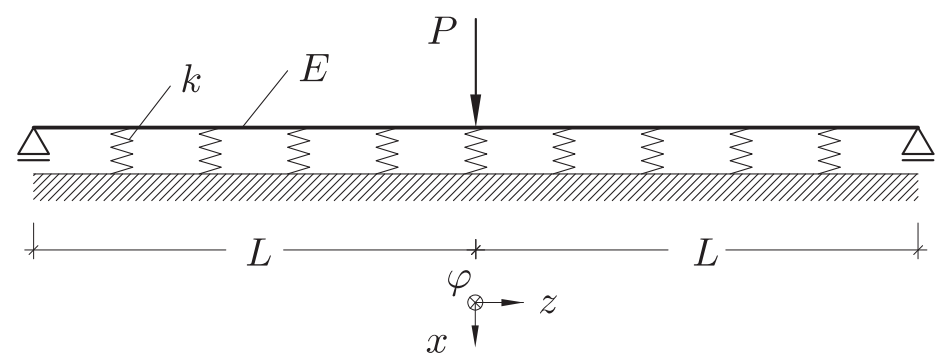

Abbildung 4.2: Elastisch gebettete Kreisplatte unter einer in Feldmitte angreifenden Last $P$

am Rand naviergelagerten Kreisplatte mit dem Radius $L$ nach Gleichung 4.6 normiert und in Abbildung 4.3 über dem Verhältnis $f / L$ aufgetragen, das sich aus

$$
\frac{f}{L}=\frac{R}{L}-\sqrt{\left(\frac{R}{L}\right)^{2}-1}
$$

ergibt.

$$
u_{P}=\frac{(3+\nu) P L^{2}}{16 \pi(1+\nu)} \cdot \frac{1-\nu^{2}}{E t^{3}}
$$

Für sehr kleine $f / L$, wenn also die elastische Bettung der Platte sehr klein ist, nähert sich die Durchbiegung der elastisch gebetteten Platte $u_{P, k}$ der Durchbiegung der ungebetteten Platte $u_{P}$ an. Das bedeutet, die Lastabtragung erfolgt hauptsächlich über Biegung, wie es beispielsweise bei sehr flachen Kugelkappen der Fall ist. Für große $f / L$ ist dagegen die Bettung der Kreisplatte sehr groß. Dies ist mit steilen Kugelkappen gleichzusetzen, bei denen die Lastabtragung hauptsächlich über die Membrantragwirkung erfolgt.

\subsection{Einfluss der Geometrieparameter auf das Last- Verformungsverhalten am Beispiel der Kugel- kappe und des Stabzweischlages}

Wie die analytischen und experimentellen Untersuchungen [9] bis [18] gezeigt haben, besteht ein Zusammenhang zwischen der Schlankheit $\lambda$ und der kritischen 


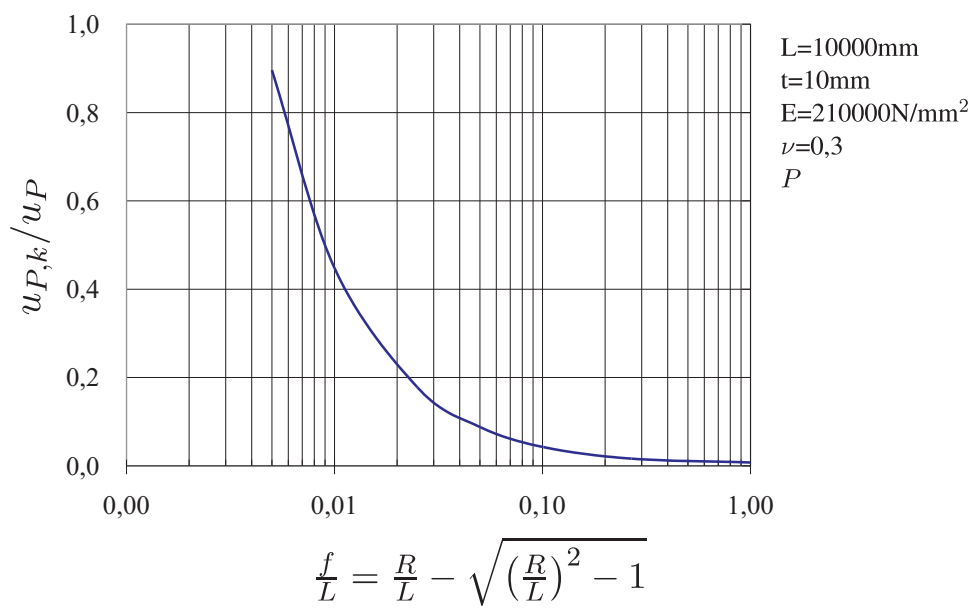

Abbildung 4.3: Durchbiebung $u_{P, k} / u_{P}$ in Abhängigkeit von $f / L$

Last $P_{\text {crit }}$ der Kugelkappe unter lokaler Belastung. In Abbildung 4.4 sind die Last-Verformungskurven von Kugelkappen unter einer am Scheitel angreifenden Einzellast $P$, wie sie in Abbildung 4.1 dargestellt ist, aus den analytischen Untersuchungen von Mescall [14] aufgetragen.

Die Kugelkappe ist am unteren Rand in vertikaler Richtung gehalten und wird im Scheitel mit einer radial gerichteten Punktlast $P$ belastet. Da sehr große Verformungen auftreten, wirken sich nichtlineare Effekte auf das Tragverhalten der Kugelkappe aus. In der Last-Verformungsbeziehung zeigt sich dies dadurch, dass die Kurven zunächst linear ansteigen und im Bereich großer Verformungen nichtlinear werden. Mit steigendem $\lambda$ bildet sich ein lokales Maximum in der LastVerformungskurve aus, das heißt, die Kugelkappe schlägt durch. Das Lastniveau, auf dem dieses Maximum auftritt, ist umso höher, je größer $\lambda$ ist.

Im Gegensatz zu Mescalls Kugelkappen unter einer Punktlast, erfolgt in der Realität die Lasteinleitung über einen Stutzen oder ein Rohr mit einem Durchmesser $d_{r}$. In den Untersuchungen von Chien [10] und Penning [15] wurden zwar Kugelkappen mit unterschiedlichen Lasteinleitungsdurchmessern $d_{r}$ berücksichtigt, inwiefern sich dies auf das Last-Verformungsverhalten auswirkt, ist dort allerdings nicht dokumentiert. Der Einfluss des Stutzendurchmessers $d_{r}$ auf das Tragverhalten wird im Folgenden in einer Studie mit der FE-Methode unter Berücksichtigung der geomerischen Nichtlinearität und elastischem Werkstoffgesetz untersucht. Dabei wird in Anlehnung an die Arbeit von Bijlaard [4] der Stutzendurch- 


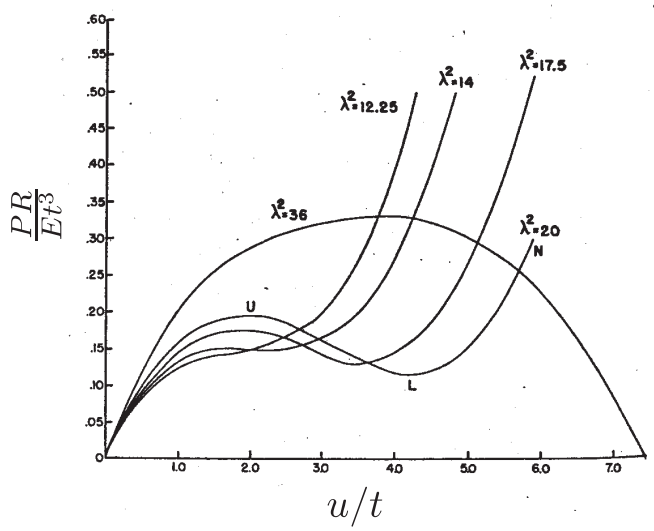

Abbildung 4.4: Last-Verformungskurven aus [14]

messer in dimensionsloser Form mit

$$
d^{*}=\frac{d_{r}}{\sqrt{R t}}
$$

ausgedrückt und ist somit eine Funktion der Abklinglänge. Neben dem Einfluss dieses bezogenen Stutzendurchmessers $d^{*}$ wird auch der Einfluss des $R / t$-Verhältnisses sowie des Geometrieparameters $\lambda$ untersucht. In den FEUntersuchungen werden vertikal gelagerte Kugelkappe betrachtet, wobei die Last $P$ am Scheitel als gleichförmige Flächenlast auf eine kreisrunde Fläche mit dem Durchmesser $d_{r}$ eingeleitet wird. Die Kugelkappe wird mit dem FE-Modell KFV der Kugelkappe mit einer Lasteinleitungsfläche nach Abschnitt 3.3.2 idealisiert.

\section{Einfluss des $R / t$-Verhältnisses}

Zunächst wurde der Einfluss des $R / t$-Verhältnisses auf das LastVerformungsverhalten der Kugelkappe untersucht. Die Abmessungen der Kugelkappen nach Abbildung 3.1 (a) und (b) wurden so variiert, dass $\lambda$ und $d^{*}$ konstant bleiben. Die tatsächlichen Abmessungen und die bezogenen Größen sind in Tabelle 4.1 aufgeführt.

Die Last-Verformungskurven aus den entsprechenden Berechnungen zeigt Abbildung 4.5. In der dimensionslosen Darstellung wurde die aufgebrachte 


\begin{tabular}{|c||c|c|c|c|c|c|c|}
\hline$j$ & $R$ & $t$ & $L$ & $d_{r}$ & $R / t$ & $\lambda$ & $d^{*}$ \\
\hline & $\mathrm{mm}$ & $\mathrm{mm}$ & $\mathrm{mm}$ & $\mathrm{mm}$ & - & - & - \\
\hline \hline 1 & 254 & 0,99 & 72 & 36 & 256 & 8,2 & 2,26 \\
\hline 2 & 508 & 0,99 & 102 & 51 & 512 & 8,2 & 2,26 \\
\hline 3 & 1016 & 0,99 & 144 & 72 & 1024 & 8,2 & 2,26 \\
\hline 4 & 2032 & 0,99 & 203 & 101 & 2050 & 8,2 & 2,26 \\
\hline 5 & 5151 & 0,99 & 583 & 161 & 5100 & 8,2 & 2,26 \\
\hline
\end{tabular}

Tabelle 4.1: Variationsparameter der Kugelkappe für unterschiedliche $R / t$ Verhältnisse

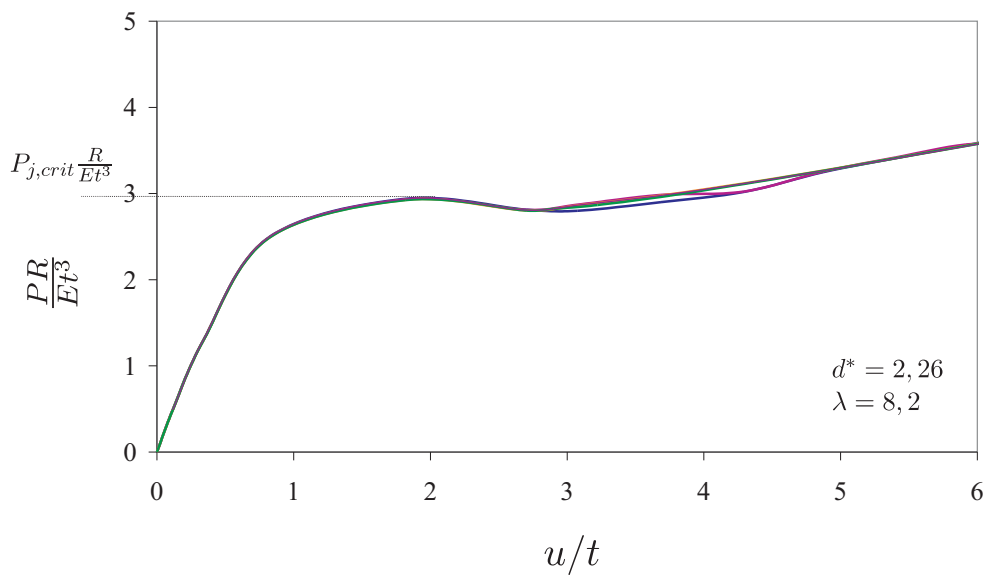

Abbildung 4.5: Last-Verformungskurven von Kugelkappen mit $d^{*}=2,26, \lambda=8,2$ und $256 \leq R / t \leq 5100$

Last $P$ mit $\frac{R}{E t^{3}}$ multipliziert und über der mit der Wanddicke $t$ normierten radialen Verschiebung $u$ an der Lasteinleitungsstelle aufgetragen. Dabei zeigt sich, dass bei veränderlichem $R / t$-Verhältnis mit konstantem $\lambda$ und $d^{*}$ die Last-Verformungskurven identisch sind. Das lokale Maximum der LastVerformungskurven ist die dimensionslose kritische Last $P_{j, c r i t} \frac{R}{E t^{3}}$, die folglich auch unabhängig von $R / t$ ist. 


\section{Einfluss des Schlankheitsgrades $\lambda$}

In einer weiteren Studie wurde der Schlankheitsgrad $\lambda$ der Kugelkappen nach Abbildung 3.1 (a) und (b) variiert, indem bei konstantem $t$ der Radius $R$ und der Grundkreisradius $L$ verändert wurde. Da der bezogene Durchmesser $d^{*}$ konstant sein muss, wurde $d_{r}$ entsprechend angepasst (vgl. Tabelle 4.2). Da $R / t$ keinen Einfluss auf das Last-Verformungsverhalten hat, kann dieser Wert beliebig sein. Als Ergebnis dieser Berechnungen sind die Last-Verformungskurven in Abbildung 4.6 aufgetragen.

\begin{tabular}{|c||c|c|c|c|c|c|c|}
\hline$j$ & $R$ & $t$ & $L$ & $d_{r}$ & $R / t$ & $\lambda$ & $d^{*}$ \\
\hline & $\mathrm{mm}$ & $\mathrm{mm}$ & $\mathrm{mm}$ & $\mathrm{mm}$ & - & - & - \\
\hline \hline 1 & 254 & 0,99 & 102 & 36 & 256 & 11,6 & 2,26 \\
\hline 2 & 508 & 0,99 & 102 & 51 & 512 & 8,2 & 2,26 \\
\hline 3 & 1016 & 0,99 & 85 & 72 & 1024 & 4,9 & 2,26 \\
\hline 4 & 254 & 0,99 & 37 & 36 & 256 & 4,2 & 2,26 \\
\hline
\end{tabular}

Tabelle 4.2: Variationsparameter der Kugelkappe für unterschiedliche $\lambda$

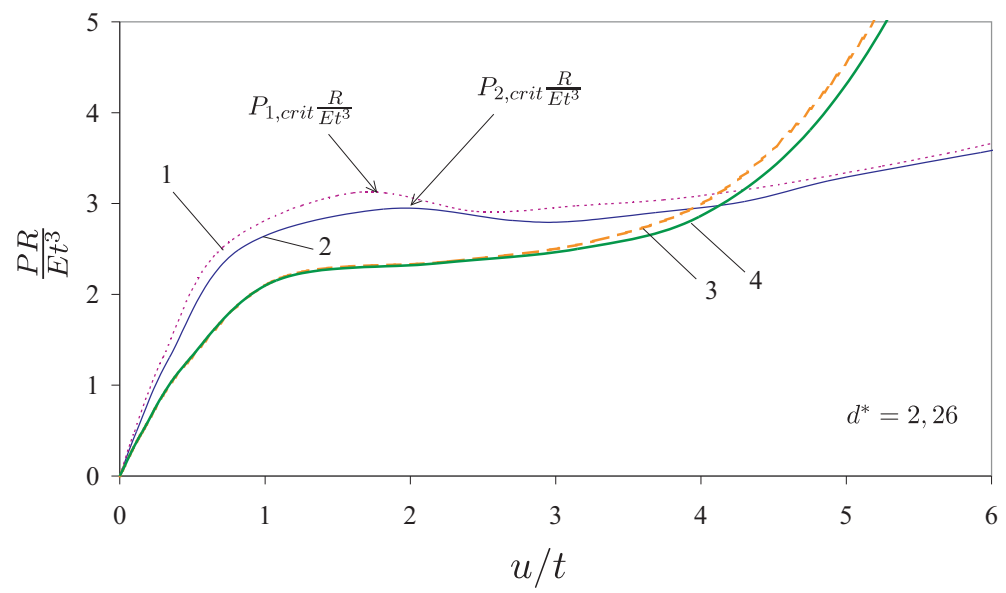

Abbildung 4.6: Last-Verformungskurven von Kugelkappen mit $d^{*}=2,26$, $4,2 \leq \lambda \leq 11,6$ und beliebigen $R / t$-Verhältnissen

Im dargestellten Beispiel tritt für $\lambda<8,2$ kein lokales Maximum in den LastVerformungskurven auf. Nur Kugelkappen, deren Schlankheitsgrad $\lambda$ größer als 8,2 ist, haben ein ausgeprägtes lokales Maximum im Kurvenverlauf, welches die 
kritische Last $P_{j, \text { crit }}$ repräsentiert. Mit dem Schlankheitsgrad nehmen auch die kritischen Lasten der Kugelkappen zu. Dies bestätigt das Ergebniss der analytischen Untersuchungen von Mescall in [14].

In Abbildung 4.7 sind die Last-Verformungskurven von Kugelkappen in Biegeund Membrananteil aufgeteilt, qualitativ dargestellt. Die Last-Verformungskurven der Kugelkappen mit dominantem Biegeanteil (Kugelkappe 1) und dominantem Membrananteil (Kugelkappe 2) ergeben sich aus der unterschiedlichen Wichtung der Biege- und Membrananteile zueinander. Die Charakteristik der LastVerformungskurven ist dabei abhängig vom Verhältnis des Membran- zum Biegeanteil und somit vom Schlankeitsgrad $\lambda$. Wird die Last hauptsächlich über die Membrantragwirkung abgetragen, so haben die Last-Verformungskurven ein lokales Maximum (Kugelkappe 2). In diesem Fall ist der Schlankheitsgrad $\lambda$ der Kugelkappe größer als bei Kugelkappen, welche die Last hauptsächlich über Biegung abtragen (Kugelkappe 1). Die Last-Verformungskurven von Kugelkappen mit kleinem $\lambda$ verlaufen dagegen monoton steigend, haben also kein lokales Maximum.

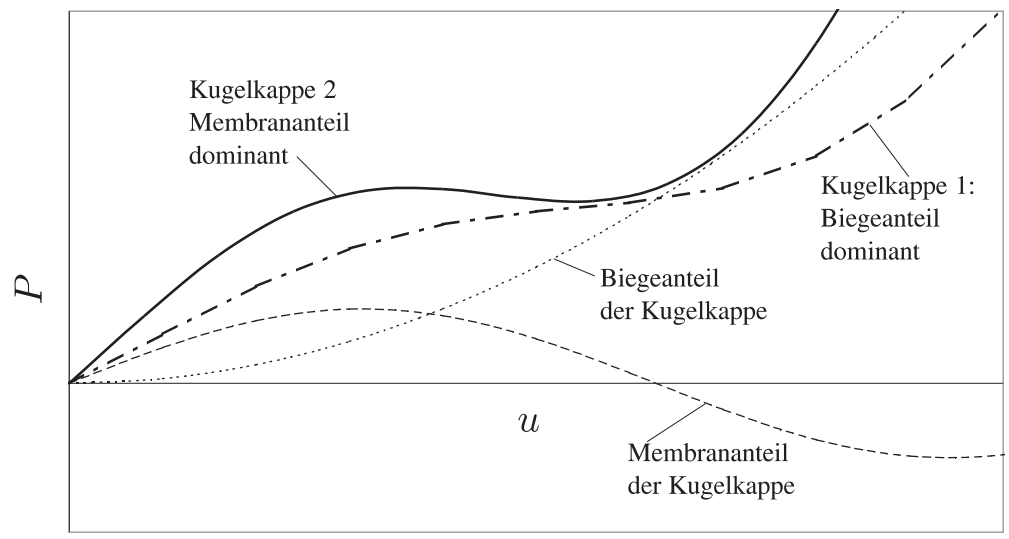

Abbildung 4.7: Last-Verformungskurven von Kugelkappen in Membran- und Biegeanteil aufgeteilt

\section{Einfluss des bezogenen Durchmessers $d^{*}$}

Im nächsten Schritt wurde der Einfluss des bezogenen Durchmessers $d^{*}$ auf das Tragverhalten der Kugelkappe untersucht. In Abbildung 4.8 sind die Last- 
Verformungskurven von Kugelkappen mit unterschiedlichen $d^{*}$ bei einem konstanten Schlankheitsgrad $\lambda$ dargestellt. Das $R / t$-Verhältnis kann dabei beliebig sein. Die Abmessungen der untersuchten Kugelkappen sind in Tabelle 4.3 aufgeführt.

\begin{tabular}{|c||c|c|c|c|c|c|c|}
\hline$j$ & $R$ & $t$ & $L$ & $d_{r}$ & $R / t$ & $\lambda$ & $d^{*}$ \\
\hline & $\mathrm{mm}$ & $\mathrm{mm}$ & $\mathrm{mm}$ & $\mathrm{mm}$ & - & - & - \\
\hline \hline 1 & 1016 & 0,99 & 85 & 72 & 1024 & 8,2 & 3,00 \\
\hline 2 & 508 & 0,99 & 102 & 51 & 512 & 8,2 & 2,26 \\
\hline 3 & 508 & 0,99 & 102 & 25 & 512 & 8,2 & 1,13 \\
\hline
\end{tabular}

Tabelle 4.3: Variationsparameter der Kugelkappe für unterschiedliche $d^{*}$

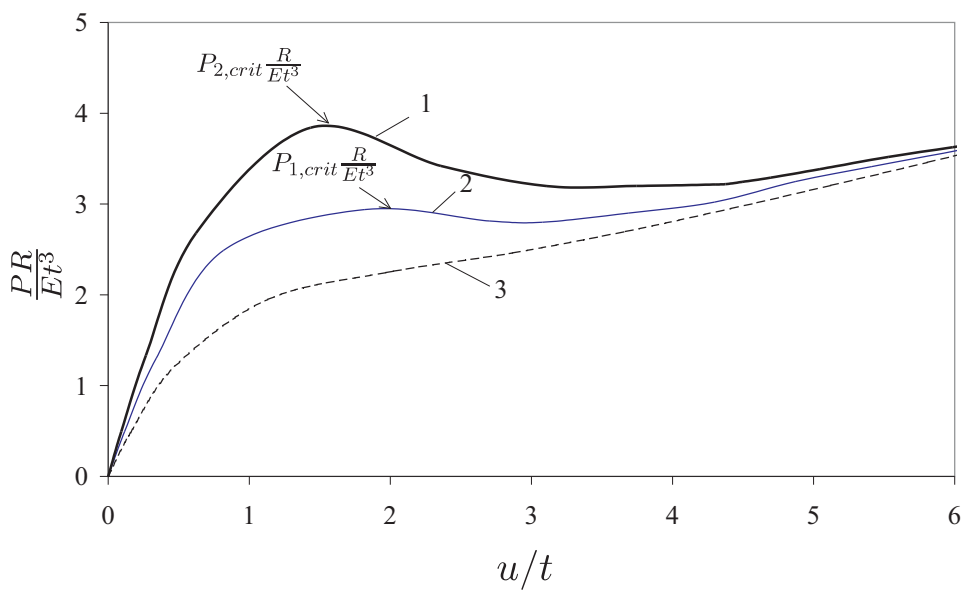

Abbildung 4.8: Last-Verformungskurven von Kugelkappen mit $\lambda=8,2$, $1,13 \leq d^{*} \leq 3,0$ und beliebigem $R / t$

Wie Abbildung 4.8 zeigt, hat die Last-Verformungskurve der Kugelkappe mit $d^{*}=1,13$ kein lokales Maximum. Erst für $d^{*}>2,26$ tritt ein lokales Maximum in der Last-Verformungskurve auf. Mit zunehmendem $d^{*}$ steigt auch die kritische Last $P_{j, \text { crit }} \frac{R}{E t^{3}}$ der Kugelkappe. Das unterschiedliche Tragverhalten in Abhängigkeit von $d^{*}$ steht ebenfalls im Zusammenhang mit der Biege- und Membrantragwirkung der Struktur. In Abhängigkeit vom Durchmesser der Lasteinleitungsfläche $d^{*}$ kann dies anschaulich am Beispiel der zwei Kugelkappen in Abbildung 4.9 mit unterschiedlichem $d_{r}$ bei gleichem $R, t$ und $L$ erklärt werden. Wird die am Scheitel der Kugelkappe angreifende Flächenlast $P$ in Lastkomponenten 
$P_{\perp}$ rechtwinklig und $P_{\|}$parallel zur Schalenmittelfläche zerlegt, so wird deutlich, dass bei der Kugelkappe mit dem größeren $d_{r}$ deutlich mehr Lastkomponenten parallel zur Schalenmittelfläche vorhanden sind, als bei der Kugelkappe mit dem kleineren $d_{r}$. Je größer $d_{r}$ (bei konstantem $\lambda$ ) ist, umso mehr parallele Lastkomponenten $P_{\| \mid}$sind vorhanden und umso mehr beteiligen sich die Membrankräfte an der Lastabtragung. Im Grenzfall, wenn die Last über einen singulären Punkt mit $d_{r} \rightarrow 0$ eingeleitet wird, sind nur rechtwinklige Lastkomponenten $P_{\perp}$ vorhanden, so dass die Lastabtragung hauptsächlich über Biegung erfolgt.

(a)

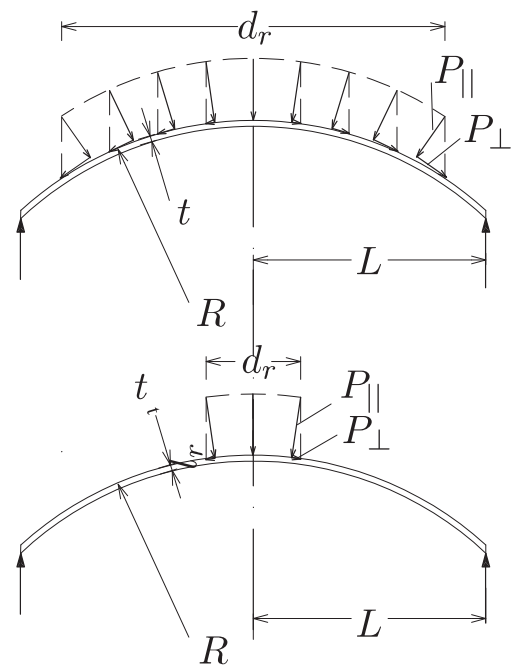

Abbildung 4.9: Radiale Last aufgeteilt in parallelle $P_{\|}$und rechwinklige $P_{\perp}$ Lastkomponenten für (a) große $d_{r}$ und (b) kleine $d_{r}$ 


\section{Analogie zum Stabzweischlag}

Das Tragverhalten einer Kugelkappe in Abhängigkeit von den Biege- und Membrananteilen kann am Beispiel des Stabzweischlages analytisch dargestellt werden. Dazu wird der Stabzweischlag nach Abbildung 4.10 mit der Neigung $\alpha$ und der projizierten Schenkellänge $L$ betrachtet, der im Scheitelgelenk mit der Kraft $P$ belastet wird. Die beiden Schenkel des Stabzweischlages sind über eine Drehfeder mit der Drehfedersteifigkeit $K$ verbunden. Für eine endliche Dehnsteifigkeit $E A$ der Stäbe und eine sehr große Biegsteifigkeit $(E I \rightarrow \infty)$ kann die Kraft $P$ in Abhängigkeit vom Absenkwinkel $\varphi$ mit

$$
P=\underbrace{2 E A \tan (\alpha-\varphi)[\cos (\alpha-\varphi)-\cos \alpha]}_{\text {Membrananteil }}+\underbrace{\frac{4 K}{L} \cdot \frac{\varphi}{\cos (\alpha-\varphi)}}_{\text {Biegeanteil }}
$$

ermittelt werden.

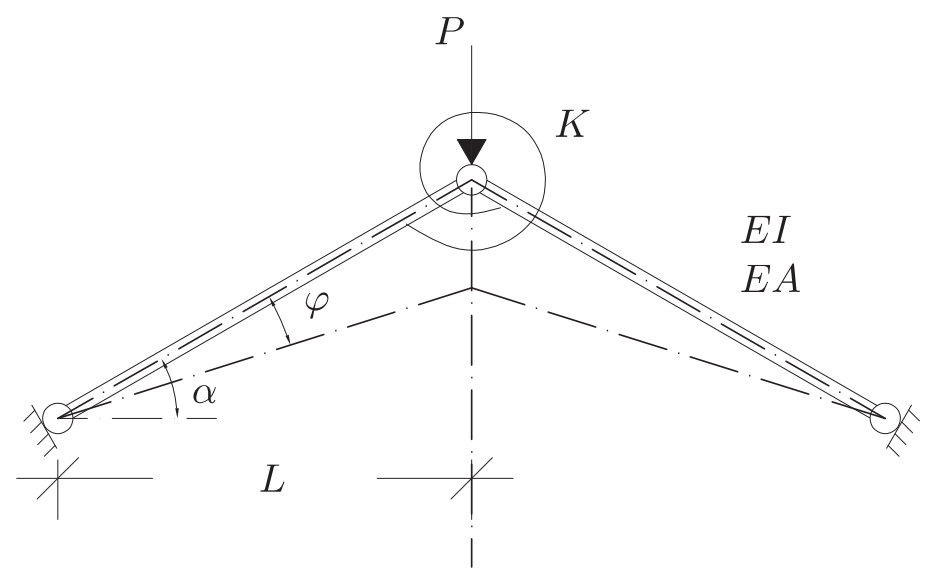

Abbildung 4.10: Stabzweischlag mit Drehfeder

Wie bei der Kugelkappe wird das Last-Verformungsverhalten von zwei Anteilen, die sich an der Lastabtragung beteiligen, bestimmt. Der erste Term in Gleichung 4.8 repräsentiert die Dehnsteifigkeit des Stabes (Membrananteil). Der zweite Term beschreibt den Anteil der Drehfeder (Biegeanteil). Durch Variation der Parameter $K, \alpha, L$ sowie der Dehnsteifigkeit $E A$ verändert sich das Verhältnis 
der beiden Beiträge zur Kraft $P$. Stehen diese beiden Anteile in einem bestimmten Verhältnis zueinander, so schlägt die Struktur bei der Last $P_{\text {crit }}$ durch. In Abbildung 4.11 ist die mit der Dehnsteifigkeit $E A$ normierte Last nach Gleichung 4.8 über dem normierten Absenkwinkel $\varphi / \alpha$ für unterschiedliche Neigungswinkel $\alpha$ bei konstanter Drehfedersteifigkeit $K$ aufgetragen. Dabei wird deutlich, dass die Durchschlaglast $P_{\text {crit }}$ umso höher ist, je steiler der Stabzweischlag ist. Für kleine Neigungswinkel $\alpha$ sind die Last-Verdrehungskurven dagegen monoton steigend. Wie anhand der relativen Drehfederanteile aus Tabelle 4.4 klar gezeigt werden kann, kommt es im dargestellten Beispiel zum Durchschlagen des Stabzweischlages, wenn der Federanteil kleiner als etwa 55\% ist. Das heißt, bei einem steilen Stabzweischlag ist der Anteil der Drehfeder klein gegenüber dem Stabanteil und die Struktur schlägt bei einer Last $P_{\text {crit }}$ durch. Setzt man den Drehfederanteil des Stabzweischlages dem Biegeanteil der Kugelkappe gleich, so wird die Analogie des Tragverhaltens dieser beiden Strukturen deutlich.

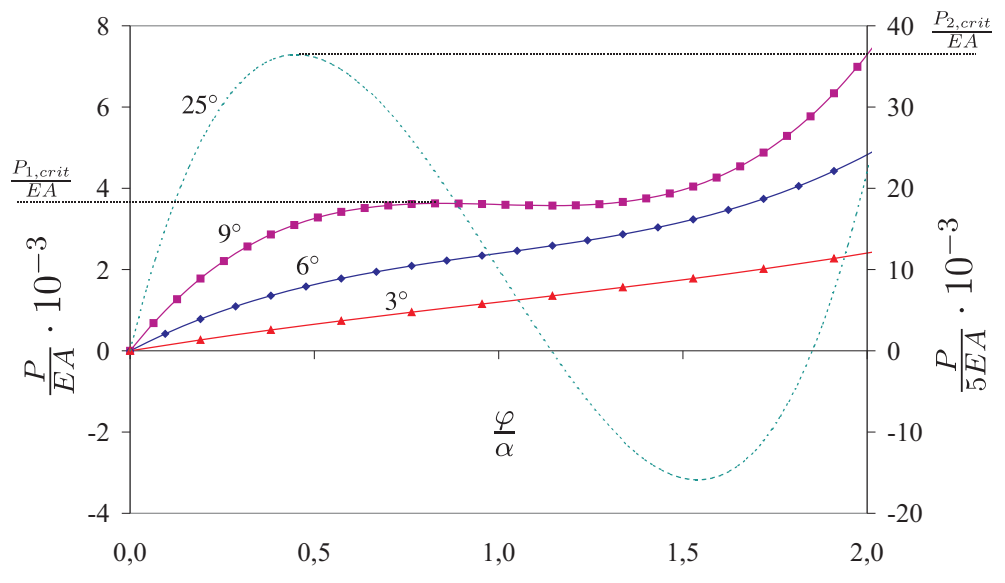

Abbildung 4.11: Last-Verdrehungskurven des Stabzweischlages mit unterschiedlichen Neigungen

Die Schlussfolgerungen dieser Analogiebetrachtung sind in Tabelle 4.5 aufgeführt und untermauern die Ergebnisse aus Abbildung 4.6 und Abbildung 4.11. Der Schlankheitsgrad beider Strukturen ist eine Funktion der geometrischen Abmessungen. Beim Stabzweischlag sind dies der Neigungswinkel $\alpha$, die projizierte Schenkellänge $L$ und die Fläche $A$ des Stabes. Bei der Kugelkappe hängt der Schlankheitsgrad $\lambda$ vom Radius $R$, vom Basiskreisradius $L$ und von der Wand- 


\begin{tabular}{|c||c|c|c|c|c|c|}
\hline$\alpha$ & $L$ & $E$ & $A$ & $K$ & $\begin{array}{c}\text { Feder- } \\
\text { anteil }\end{array}$ & $\mathrm{P}_{\text {crit }} / E A$ \\
\hline$[-]$ & $\mathrm{mm}$ & $\mathrm{N} / \mathrm{mm}^{2}$ & $\mathrm{~mm}^{2}$ & $\mathrm{Nmm}$ & $\%$ & - \\
\hline \hline $3^{\circ}$ & 2000 & 210000 & 1500 & $6,3 \cdot 10^{7}$ & 85 & - \\
\hline $6^{\circ}$ & 2000 & 210000 & 1500 & $6,3 \cdot 10^{7}$ & 55 & - \\
\hline $9^{\circ}$ & 2000 & 210000 & 1500 & $6,3 \cdot 10^{7}$ & 34 & $3,63 \cdot 10^{-4}$ \\
\hline $25^{\circ}$ & 2000 & 210000 & 1500 & $6,3 \cdot 10^{7}$ & 6 & $3,65 \cdot 10^{-3}$ \\
\hline
\end{tabular}

Tabelle 4.4: Abmessungen der untersuchten Stabzweischläge

dicke $t$ ab. Verändert man eine dieser Größen, so wirkt sich dies auf das Verhältnis der Lastanteile aus. Unter der Annahme, dass der Neigungswinkel $\alpha$ des Stabzweischlages variabel ist und die restlichen Größen konstant sind, vergrößert sich bei zunehmendem $\alpha$ der Stabanteil gegenüber dem Drehfederanteil. Die Lastabtragung erfolgt dann hauptsächlich über den Stabanteil. Je steiler der Neigungswinkel $\alpha$ ist, desto größer ist die Durchschlagkraft $P_{\text {crit }}$. Im Fall der Kugelkappe ist der Neigungswinkel über die Beziehung $\cos \alpha=L / R$ gegeben. Auch in diesem Fall gilt, dass bei großen Neigungswinkeln und folglich auch großem $\lambda$ die Lastabtragung hauptsächlich über die Membrantragwirkung der Struktur erfolgt.

\subsection{Einfluss der Ausführung des Lasteinleitungsbe- reiches auf das Last-Verformungsverhalten}

Neben dem Einfluss der Steifigkeit der gesamten Kugelkappe ist auch ein Einfluss der Steifigkeit des Lasteinleitungsbereiches auf das Tragverhalten zu erwarten. Dieser Fragestellung wird im Folgenden durch Vergleichsberechnungen mit der FE-Methode unter Berücksichtigung der geometrischen Nichtlinearität und elastischem Werkstoffgesetz nachgegangen. Dazu werden eingespannte Kugelkappen unter konzentrierten Lasten, die von Penning und Thurston in [15] analytisch und experimentell untersucht wurden, betrachtet. Die Versuche wurden an Probekörpern aus Aluminium durchgeführt. Die Last wurde dabei über ein rundes Rohr in die jeweilig Kugelkappe eingeleitet. Zwischen Kugelkappe und Lasteinleitungsrohr lag eine Gummischicht, die eine Beschädigung der Schale verhindern sollte. Die analytischen Lösungen basieren auf den Arbeiten von Reissner ([1], [2], [3]). Die in [15] untersuchten Versuchskörper B33 und B35 wurden mit dem in Abschnitt 3.3.2 beschriebenen Modell KFE abgebildet. Die Abmessungen dieser Kugelkappen sind in Tabelle 4.6 aufgeführt. Diese Versuche wurden gewählt, da in den Versuchen stets axisymmetrische Verformungen auftraten. 


\begin{tabular}{|c||c|c|}
\hline & Stabzweischlag & Kugelkappe \\
\hline \hline Geometrie & $\alpha, L$ & $R, L$ \\
\hline Steifigkeit & $E, A, K$ & $E, t$ \\
\hline $\begin{array}{c}\text { Einfluss } \\
\text { auf Trag- } \\
\text { verhalten }\end{array}$ & Stabanteil & Membrananteil \\
\hline $\begin{array}{c}\text { Schlankheits- } \\
\text { grad }\end{array}$ & Drehfederanteil & Biegeanteil \\
\hline Konstante & $E, L, A)$ & $\lambda=\mathrm{f}(R, L, t)$ \\
\hline Variable & $\alpha, L$ & $E, t, d_{r}, L$ \\
\hline $\begin{array}{c}\text { Lastabtragung } \\
\text { furch }\end{array}$ & $\begin{array}{c}\text { für große } \alpha \\
\text { hauptsächlich über } \\
\text { Stabanteil }\end{array}$ & $\begin{array}{c}\text { hauptsächlich über } \\
\text { Membrananteil }\end{array}$ \\
\hline $\begin{array}{c}\text { Durchschlagen } \\
\text { tritt auf, wenn }\end{array}$ & $\begin{array}{c}\text { Stabanteil }> \\
\text { Drehfederanteil }\end{array}$ & $\begin{array}{c}\text { Membrananteil }> \\
\text { Biegeanteil }\end{array}$ \\
\hline$P_{\text {crit }}$ steigt & mit wachsendem $\alpha$ & $\begin{array}{c}\text { mit wachsendem } \lambda, \text { wobei } \\
\lambda \sim \cos \alpha\end{array}$ \\
\hline Fazit & $\begin{array}{c}\alpha \text { beeinflusst das } \\
\text { Verhältnis von Stab- und } \\
\text { Drehfederanteil } \\
\Rightarrow \text { Einfluss auf } P_{\text {crit }}\end{array}$ & $\begin{array}{c}R \text { beeinflusst das } \\
\text { Verhältnis von Membran- } \\
\text { und Biegeanteil, sowie } d^{*} \\
\Rightarrow \text { Einfluss auf } P_{c r i t}\end{array}$ \\
\hline
\end{tabular}

Tabelle 4.5: Analogie von Stabzweischlag und Kugelkappe

\begin{tabular}{|c||c|c|c|c|}
\hline & $R$ & $t$ & $L$ & $d_{r}$ \\
\hline & $\mathrm{mm}$ & $\mathrm{mm}$ & $\mathrm{mm}$ & $\mathrm{mm}$ \\
\hline \hline B33 & 508 & 0,98 & 101,6 & 3,175 \\
\hline B35 & 508 & 0,99 & 101,6 & 25,4 \\
\hline
\end{tabular}

Tabelle 4.6: Abmessungen der Kugelkappen B33 und B35

Die Last-Verformungskurven aus den analytischen und experimentellen Untersuchungen aus [15] sowie aus der FE-Berechnung der Kugelkappe B33 sind in Abbildung 4.12 aufgetragen. Es zeigt sich, dass die numerisch ermittelten LastVerformungskurven sehr gut sowohl mit den analytisch als auch mit den experimentell ermittelten Kurven übereinstimmen. Für die Kugelkappe B35 sind die Last-Verformungskurven in Abbildung 4.13 dargestellt. Dabei kommt es bereits im linearen Bereich der Last-Verformungskurve zu Abweichungen zwischen den analytischen und experimentellen Ergebnissen. Die numerisch ermittelte Last- 


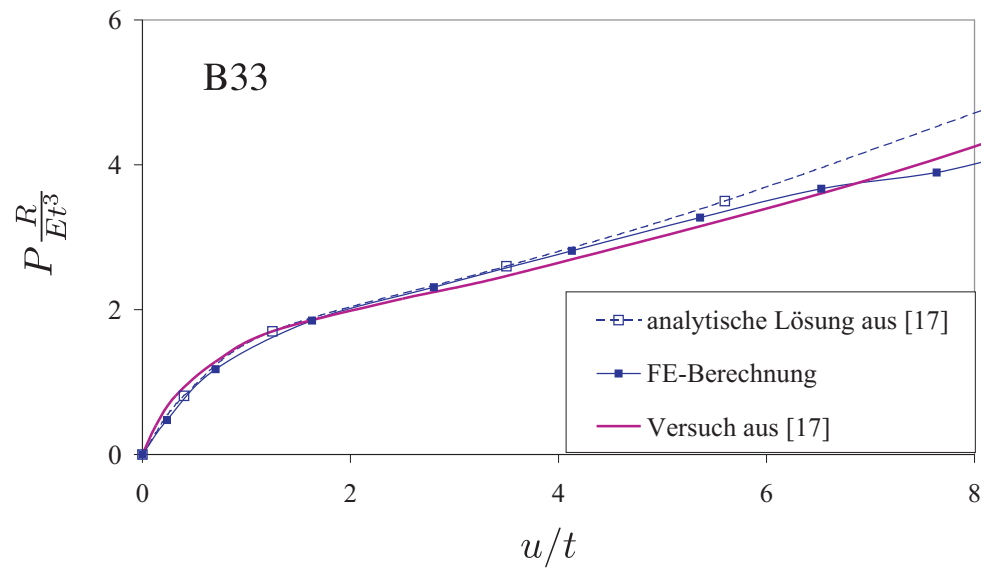

Abbildung 4.12: Last-Verformungskurven der Kugelkappe B33 aus [15] und aus der FE-Berechnung

Verformungskurve stimmt bis zu einer Last $P \frac{R}{E t^{3}}=1$ mit der im Versuch gemessen Verformungen überein. Bei größeren Lasten sind die numerisch ermittelten Verformungen jedoch größer als die gemessenen Verformungen. Die analytisch bestimmten Verformungen sind dagegen nur halb so groß, wie die numerisch ermittelten Verformungen. Die genaue Betrachtung des Versuchablaufs bei Versuchskörpern mit einem Lasteinleitungsrohr mit einem großen Durchmesser zeigt, dass sich das Rohr mit steigender Last an die Kugelkappe anschmiegt, und die Kugelkappe in diesem Bereich gewissermaßen einspannt. Diese Einspannwirkung in der Kugelkappe ist umso größer je größer der Rohrdurchmesser ist. Bei kleinen Rohrdurchmessern ensteht keine Einspannwirkung, da die Last annähernd punktuell eingeleitet wird.

Die durch das Lasteinleitungsrohr hervorgerufene Einspannwirkung wird in den numerischen Untersuchungen durch eine erhöhte Steifigkeit der Lasteinleitungsbereichs berücksichtigt. Dazu wird der Teil der Kugelkappe, in den die Flächenlast eingeleitet wird, mit einer größeren Wanddicke modelliert. In Abbildung 4.14 ist die Last-Verformungskurve der Kugelkappe B35 mit einer um 20\% größeren Wanddicke im Lasteinleitungskreis sowie die im Versuch gemessene LastVerformungskurve aufgetragen. Mit diesem Modell der Kugelkappe wird eine sehr gute Übereinstimmung der FE-Ergebnisse mit der experimentellen LastVerformungskurve erzielt. 


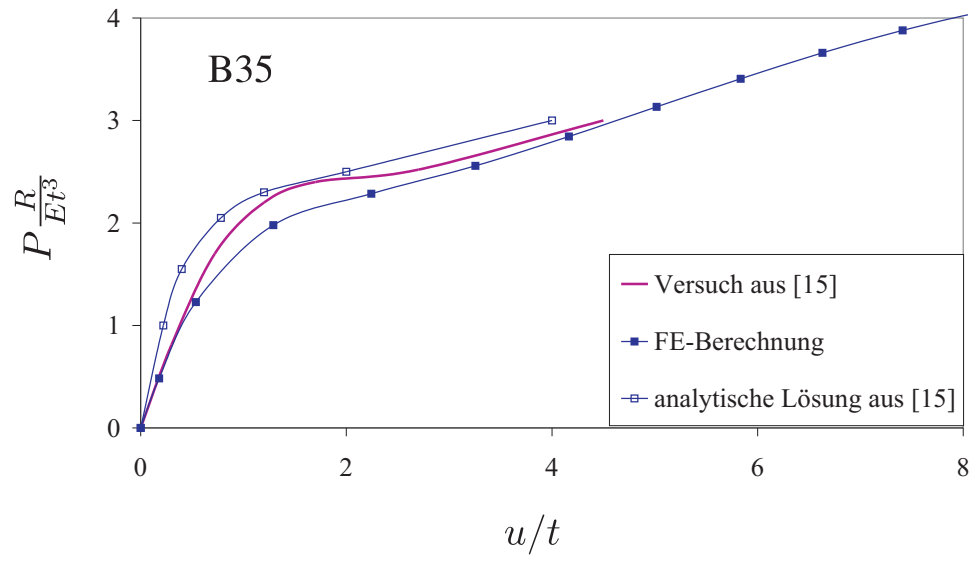

Abbildung 4.13: Last-Verschiebungskurven der Kugelkappe B35 aus [15] und aus einer FE-Berechnung

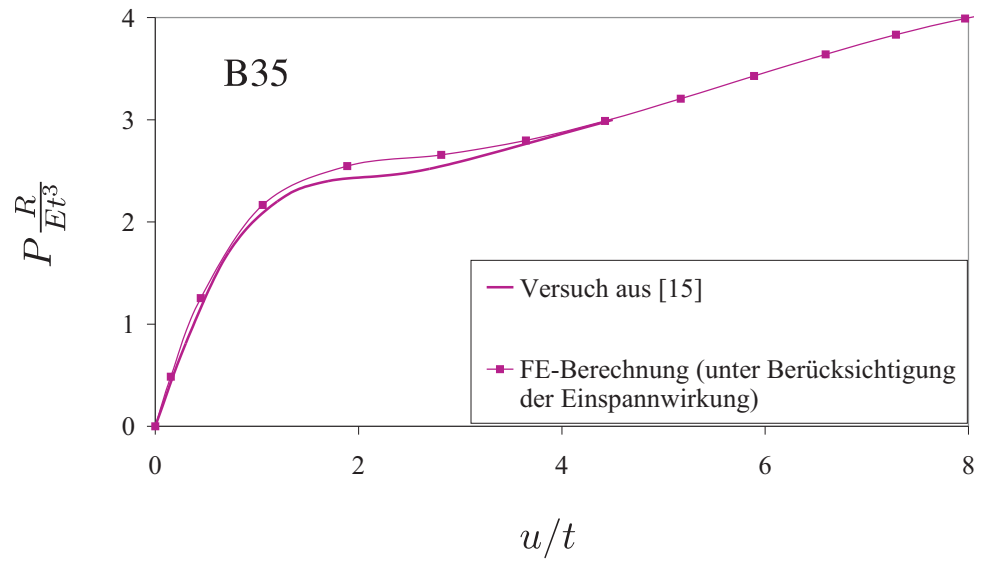

Abbildung 4.14: Versuchskurve und Last-Verschiebungskurve der Kugelkappe B35 mit einer Wanddicke von 1,2t zur Modellierung der Einspannwirkung im Lasteinleitungsbereich 


\subsection{Einfluss der Symmetrierandbedingungen auf das Last-Verformungsverhalten}

Werden in numerischen Untersuchungen Strukturen unter Ausnutzung der Symmetrieeigenschaften dargestellt, so besteht die Möglichkeit, dass asymmetrische Verformungen durch die Symmetrierandbedingungen verhindert werden. Wie sich in den experimentellen Untersuchungen von Penning und Thurston [15] an Kugelkappen aus Aluminium unter einer im Scheitel angreifenden Last gezeigt hatte, können asymmetrische Beulmuster auftreten (vgl. Abschnitt 2.2). Wird für die numerische Berechnung dieser Kugelkappen ein $90^{\circ}$-Segment der Kugelkappe verwendet, wie es in Abschnitt 3.3 beschrieben wurde, so können die Symmetriebedingungen die Ausbildung asymmetrische Beulfiguren verhindern. Durch vergleichende Berechnung der in [15] dokumentierten Versuche mit einem $90^{\circ}$ Segment sowie einer kompletten Kugelkappe $\left(360^{\circ}\right)$ wird der Frage nachgegangen, inwiefern die Symmetrierandbedingungen das Tragverhalten der Kugelkappe beeinflussen. Für die Vergleichsberechnungen wurde die Kugelkappe B43 aus [15] mit einem Verhältnis des Durchmessers der Lasteinleitung $d_{r}$ zum Grundkreisradius $L$ der Kugelkappe von 1/8 herangezogen. Die Abmessungen dieser Kugelkappe sind in Tabelle 4.7 aufgeführt.

\begin{tabular}{|c|c|c|c|c|}
\hline$R$ & $t$ & $L$ & $d_{r}$ & E-Modul \\
\hline $\mathrm{mm}$ & $\mathrm{mm}$ & $\mathrm{mm}$ & $\mathrm{mm}$ & $\mathrm{N} / \mathrm{mm}^{2}$ \\
\hline \hline 508 & 0,259 & 101,6 & 25,4 & 69000 \\
\hline
\end{tabular}

Tabelle 4.7: Abmessungen der Kugelkappe aus Versuch B43 nach [15]

Für die FE-Berechnungen wurde die am unteren Rand eingespannte Kugelkappe mit dem in Abschnitt 3.3.2 beschriebenen 90 -Modell (KFE) abgebildet. Zur Berücksichtigung der Einspannwirkung, die das Lasteinleitungsrohr auf die $\mathrm{Ku}-$ gelkappe ausübt, wurde im Lasteinleitungsbereich die Wanddicke $t$ um $10 \%$ vergrößert.

In einer zweiten Berechnung wurde die Kugelkappe als $360^{\circ}$-Modell abgebildet, das mit Ausnahme der Symmetrieränder dem in Abschnitt 3.3.2 beschriebenen $90^{\circ}$-Modell (KFE) entspricht. Die Last-Verformungskurven der numerischen Untersuchungen an einem $90^{\circ}$ - und $360^{\circ}$-Segment sowie die experimentell ermittelte Last-Verformungskurve aus [15] sind in Abbildung 4.15 aufgetragen. In den experimentellen Untersuchungen trat bei einer normierten Last $P \frac{R}{E t^{3}} \approx 2,7$ das erste Stabilitätsproblem in Form einer symmetrischen Beule auf. Die Nebenpfade der Kurve sind in Abbildung 4.15 gestrichelt eingezeichnet. Bei weiterer Laststeigerung verformte sich die Schale weiter symmetrisch, bis sich bei einer normierten 
Last von etwa 7 eine dreieckförmige Verformungsfigur ausbildete. Das zweite Beulen trat bei einer Last von etwa 8,4 auf. Dabei ging das dreieckige Beulmuster in ein viereckiges und bei weiterer Laststeigerung in ein fünfeckiges Beulmuster über.

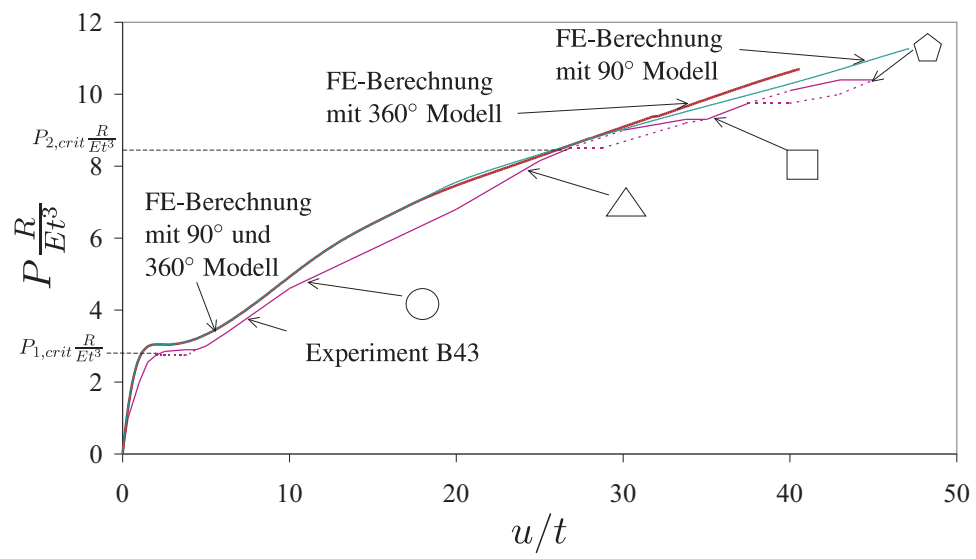

Abbildung 4.15: Vergleich der Last-Verformungskurve der Kugelkappe aus Versuch B43 $\left(d_{r}=25,4 \mathrm{~mm}\right)$ mit den Last-Verformungskurven des $90^{\circ}$ - und $360^{\circ}$-FEModells

Der Verlauf der Last-Verformungskurven aus den FE-Berechnungen mit dem $90^{\circ}$ Segment und dem $360^{\circ}$ Modell ist bis zu einer Last $P \frac{R}{E t^{3}}$ von etwa 7 vollkommen identisch. Bei $P \frac{R}{E t^{3}} \approx 3,0$ tritt ein Stabilitätsproblem auf. Die Verformungsfigur beider Modelle ist bis zu einer Last $P \frac{R}{E t^{3}}$ von etwa 7 kreisförmig, d.h. rotationssymmetrisch. Bei größeren Lasten bildet sich im $360^{\circ}$-Modell der Kugelkappe eine dreiecksförmige Verformungsfigur aus, die bis zum Ende der Berechnung $\left(P \frac{R}{E t^{3}}=11\right)$ dreieckförmig blieb. Beim $90^{\circ}$-Segment wird dagegen die Ausbildung einer dreickigen Verformungsfigur durch die Symmetrierandbedingungen verhindert. Daher ging bei diesem Modell die kreisförmige Verformungsfigur ab einer Last $P \frac{R}{E t^{3}}$ von etwa 7 in eine viereckige Verformungsfigur über. Es hat sich gezeigt, dass im Vorbeulbereich sowie über den ersten Stabilitätspunkt hinweg bis zu einer Last $P \frac{R}{E t^{3}}$ von etwa 7 nur rotationssymmetrische Verformungen in der Kugelkappe auftraten. Außerdem stimmten die Last-Verformungskurven des $90^{\circ}$-Segments und der kompletten Kugelkappe über den ersten Stabilitätspunkt hinweg vollkommen überein. Zudem ist die Übereinstimmung der numerisch ermittelten Kurven mit der experimentell ermittelten Kurve sehr gut. Da darüber hinaus die Symmetrierandbedingungen erst im Bereich sehr großer Verformun- 
gen $(u / t>15)$ das Last-Verformungsverhalten beeinflussen, kann davon ausgegangen werden, dass das $90^{\circ}$-Segment bis hin zu mittleren Verformungen die Kugelkappe ausreichend genau abbildet.

\subsection{Einfluss des Stutzens auf das Last- Verformungsverhalten der Kugelkappe}

In den vorangegangen Untersuchungen wurden Kugelkappen betrachtet, in die die Last als Flächenlast oder über ein nicht fest mit der Kugelkappe verbundenes, sehr steifes Rohr eingeleitet wurde. Wird die Last über einen fest mit der Kugelkappe verbundenen Stutzen eingeleitet, so können sich Einflüsse aus dem Stutzen und dessen Steifigkeit auf das Last-Verformungsverhalten der Kugelkappe ergeben. Der Einfluss der Stutzensteifigkeit auf das Tragverhalten wird untersucht, indem FE-Berechnungen an Kugelkappen mit unterschiedlichen Stutzenlängen $l_{r}$ und Stutzensteifigkeiten $E_{r}$ durchgeführt und mit den analytischen Untersuchungen von Bijlaard ([4]) verglichen werden. Die bezogene Stutzenlänge ist dabei

$$
l_{r}^{*}=\frac{l_{r}}{\sqrt{d_{r} t_{r} / 2}}
$$

In Bijlaards Arbeit werden die Auswirkungen lokaler Lasten auf das Verformungsverhalten und die Spannungen in Kugelkappen untersucht (vgl. Abschnitt 2.2). Dabei wurde der Stutzen als vollkommen starrer, in die Kugelkappen eingesetzter Zylinder betrachtet. Für die Vergleichsberechnungen wurden die vertikal gelagerten Kugelkappen mit dem Modell KSV nach Abschnitt 3.3.3 abgebildet. In geometrisch linearen Berechnungen - wegen des Vergleichs zu Bijlaards Lösungen - wurden die Kugelkappen mit den in Tabelle 4.8 aufgeführten Abmessungen unter einer im Scheitel angreifenden Stutzenlast $P \frac{R}{E t^{3}}=1$ untersucht. Die auf die Wanddicke $t$ normierten radialen Verschiebungen $u$ aus den numerischen Berechnungen sowie nach der Bijlaardschen Lösung entlang eines Meridians der Kugelkappe sind in Abbildung 4.16 aufgetragen. Dabei zeigt sich, dass die Ergebnisse der numerischen Berechnung einer Kugelkappe mit einem steifen Stutzen $\left(E_{r}=10 E\right.$ ) bei einer bezogenen Stutzenlänge $l_{r}^{*}=10$, sehr gut übereinstimmen. Ein nachgiebiger Stutzen, dessen E-Modul $E_{r}$ gleich dem E-Modul $E$ der Kugelkappe ist, führt zu radialen Verschiebungen, die um rund $15 \%$ größer sind als die radialen Verschiebungen der Kugelkappe mit einem vollkommen steifen Stutzen. Außerdem zeigt sich, dass bereits bei sehr kleinen bezogenen Stutzenlängen $\left(l_{r}^{*}<1,0\right)$ der Einfluss der Verformungen unverändert ist. Aus Abbildung 4.16 ist zudem ersichtlich, dass die großen Verformungen lokal begrenzt sind und vom Stutzen weg rasch abklingen. 


\begin{tabular}{|c|c|c|c|c|c|c|c|}
\hline$R$ & $t$ & $L$ & $E$ & $d_{r}$ & $t_{r}$ & $l_{r}$ & $E_{r}$ \\
\hline $\mathrm{mm}$ & $\mathrm{mm}$ & $\mathrm{mm}$ & $\mathrm{N} / \mathrm{mm}^{2}$ & $\mathrm{~mm}$ & $\mathrm{~mm}$ & $\mathrm{~mm}$ & $\mathrm{~N} / \mathrm{mm}^{2}$ \\
\hline \hline 10000 & 35 & 10000 & 210000 & 520 & 35 & 48 & 210000 \\
\hline 10000 & 35 & 10000 & 210000 & 520 & 35 & 95 & 210000 \\
\hline 10000 & 35 & 10000 & 210000 & 520 & 35 & 950 & 210000 \\
\hline 10000 & 35 & 10000 & 210000 & 520 & 35 & 950 & 2100000 \\
\hline
\end{tabular}

Tabelle 4.8: Abmessungen der Kugelkappen mit unterschiedlichen Stutzenlängen $l_{r}$ und Stutzensteifigkeiten $E_{r}$

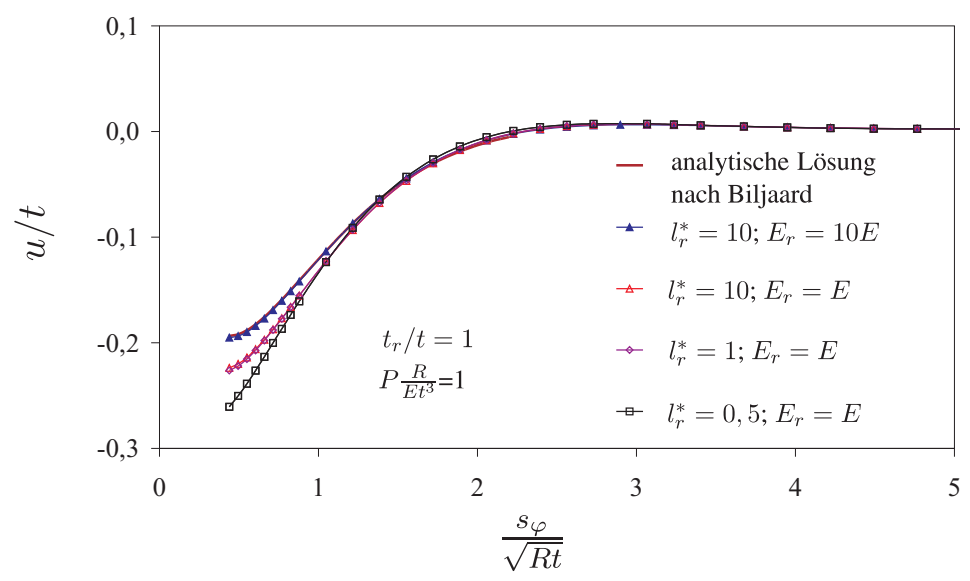

Abbildung 4.16: Radiale Verschiebungen entlang eines Meridians einer Kugelkappe unter radialer Stutzenlast

\subsection{Zusammenfassung}

Die Untersuchungen an einer Kugelkappe unter einer Einzellast haben gezeigt, dass das Tragverhalten maßgeblich vom Verhältnis der Biege- und Membrananteile beeinflust wird. Dabei besteht ein direkter Zusammenhang zwischen dem Schlankheitsgrad $\lambda$ und dem Verhältnis der Biegeanteile zu den Membrananteilen. Bei sehr schlanken Kugelkappen tritt ein Stabilitätsproblem auf, da der Membrananteil gegenüber dem Biegeanteil überwiegt. Der bezogene Stutzendurchmesser verhält sich analog dazu: Bei Kugelkappen mit großem $d^{*}$ überwiegt die Membrantragwirkung und die Last-Verformunskurven haben ein lokales Maximum. Es hat sich zudem gezeigt, dass bei Berücksichtigung nur geometrischer Nichtlinearität das $R / t$-Verhältnis keinen Einfluss auf das Tragverhalten der Ku- 
gelkappe unter radialer Einzellast hat.

Die Ausführung des Lasteinleitungsbereichs hat einen wesentlichen Einfluss auf das Last-Verformungsverhalten der Kugelkappe. Ist der Körper, mit dem die Last eingeleitet wird nicht fest mit der Kugelkappe verbunden, so spielt die Steifigkeit dieses Körpers keine Rolle. In Vergleichsberechnungen zu experimentellen und analytischen Untersuchungen hat sich allerdings gezeigt, dass ein von der Kugelkappe unabhängiges Lasteinleitungsrohr eine Einspannwirkung in der Kugelkappe erzeugt. Im FE-Modell wird diese Einspannung durch eine Erhöhung der Steifigkeit im Lasteinleitungsbereich um etwa $20 \%$ berücksichtigt. Für den Fall, dass der Stutzen fest mit der Kugelkappe verbunden ist, haben die Länge und die Steifigkeit des Stutzens einen Einfluss auf das Tragverhalten. Bei bezogenen Stutzenlängen $l_{r}^{*}>1,0$ ist dieser Einfluss jedoch bereits nicht mehr von $l_{r}^{*}$ abhängig. Die numerisch berechneten Verformungen in einer Kugelkappe mit einem eingesetzten vollkommen steifen Stutzen stimmen sehr gut mit der analytischen Lösung überein.

Die Randbedingungen, die bei Ausnutzung der Symmetrie der Kugelkappe aufgebracht werden, verhindern die Ausbildung asymmetrischer Beulformen. Da asymmetrisches Beulen erst im Bereich sehr großer Verformungen auftritt und die Auswirkungen auf das Tragverhalten zudem sehr gering sind, kann in den FE-Berechnungen bis hin zu mittleren Verformungen ein $90^{\circ}$-Segment der $\mathrm{Ku}$ gelkappe verwendet werden. 


\section{Das Tragverhalten der lokal belasteten Kreiszylinderschale}

\subsection{Hintergrund}

Der Unterschied zwischen Kugelkappe und Kreiszylinderschale besteht im wesentlichen darin, dass die Kugelkappe zwei Krümmungsrichtungen $(\varphi, z)$ aufweist, wohingegen die Kreiszylinderschale nur in eine Richtung $(\varphi)$ gekrümmt ist. Obwohl die Kreiszylinderschale unter radialer Einzellast nicht rotationssymmetrisch zur Lastachse $x$ ist, besteht eine Analogie zum Tragverhaltens der Kugelkappe. In diesem Kapitel soll gezeigt werden, dass die Faktoren, die sich auf das Tragverhalten der Kugelkappe auswirken, auch bei der Kreiszylinderschale einen Einfluss haben. Dazu werden die Einflussfaktoren, die sich auf das Tragverhalten der Kugelkappe unter Einzellast nach Abschnitt 4.7 auswirken, am Beispiel der Kreiszylinderschale unter radialer Einzellast untersucht. Zudem werden die Faktoren, die sich aus der fehlenden Krümmungsrichtung und Rotationssymmetrie ergeben, an der Kreiszylinderschale untersucht. Den numerischen Untersuchungen in diesem Kapitel liegt das Modell der elastischen Kreiszylinderschale unter radialer Einzellast $P_{x}$ und den Randbedingungen RB2 im Endquerschnitt der Kreiszylinderschale nach Abschnitt 3.4 zugrunde. Sofern nicht anders erwähnt, handelt es sich dabei um geometrisch nichtlineare Untersuchungen.

\subsection{Einfluss der Geometrieparameter $L^{*}$ und $R / t$ auf das Last-Verformungsverhalten}

Wie in Abschnitt 4.3 gezeigt wurde, hat der Schlankheitsgrad $\lambda$ einen elementaren Einfluss auf das Tragverhalten der Kugelkappe unter radialer Einzellast. Für die Kreiszylinderschale ist der bezogener Randabstand

$$
L^{*}=\frac{L}{\sqrt{R t}}
$$

eine äuqivalente Größe zum Schlankheitsgrad $\lambda$ der Kugelkappe. An Kreiszylinderschalen mit konstantem Radius $R$, konstanter Wanddicke $t$ und konstantem 
Stutzendurchmesser $d_{r}$ wurden numerische Berechnungen durchgeführt. Die Abmessungen der untersuchten Kreiszylinderschalen sind in Tabelle 5.1 aufgeführt. Abbildung 5.1 zeigt die Last-Verformungskurven der untersuchten Kreiszylinderschalen. Dort wird bestätigt, dass auch das Tragverhalten der Kreiszylinderschale deutlich vom Schlankheitsgrad $L^{*}$ beeinflusst wird.

\begin{tabular}{|c|c|c|c|c|c|c|}
\hline$R$ & $t$ & $L$ & $d_{r}$ & $R / t$ & $L^{*}$ & $d^{*}$ \\
\hline $\mathrm{mm}$ & $\mathrm{mm}$ & $\mathrm{mm}$ & $\mathrm{mm}$ & - & - & - \\
\hline \hline 10000 & 10 & 3160 & 790 & 1000 & 10 & 2,5 \\
\hline 10000 & 10 & 9487 & 790 & 1000 & 30 & 2,5 \\
\hline 10000 & 10 & 18974 & 790 & 1000 & 60 & 2,5 \\
\hline 10000 & 10 & 25300 & 790 & 1000 & 80 & 2,5 \\
\hline
\end{tabular}

Tabelle 5.1: Variationsparameter der Kreiszylinderschale für unterschiedliche $L^{*}$

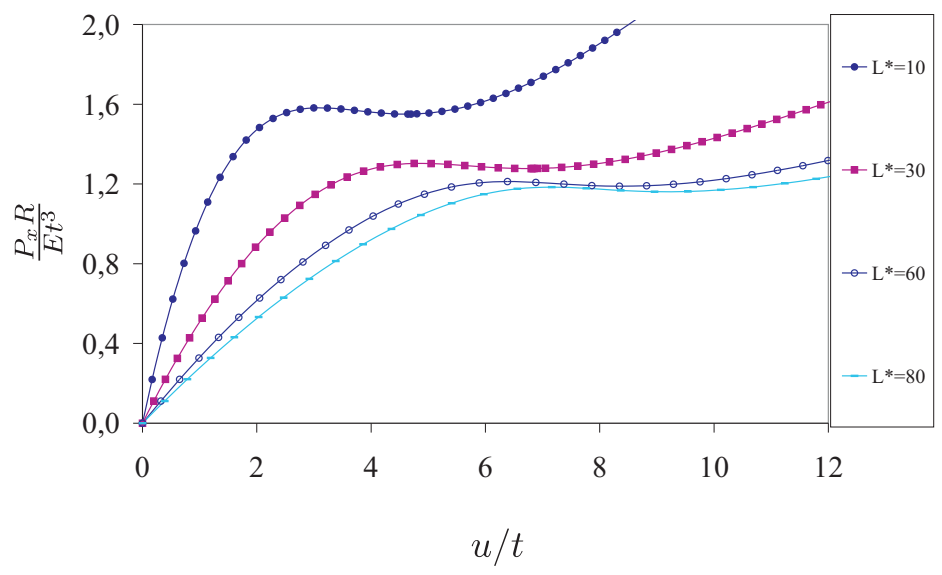

Abbildung 5.1: Last-Verformungskurven von Kreiszylinderschalen unter radialer Einzellast mit $d^{*}=2,5, R / t=1000$ und $10 \leq L^{*} \leq 80$

Im nächsten Schritt wurde der Einfluss des $R / t$-Verhältnisses auf das Tragverhalten der Kreiszylinderschale unter radialer Einzellast untersucht. Dazu wurden Kreiszylinderschalen mit konstantem $L^{*}$ betrachtet. Da sich bei der Kugelkappe gezeigt hat, dass der dimensionslose Stutzendurchmesser $d^{*}$ einen wesentlichen Einfluss auf das Tragverhalten hat, wurde in diesen Untersuchungen auch $d^{*}$ konstant gehalten. Die Abmessungen der Kreiszylinderschalen sind in Tabelle 5.2 aufgeführt. Die Last-Verformungskurven der untersuchten Kreiszylinderschalen sind in Abbildung 5.2 dargestellt. 


\begin{tabular}{|c|c|c|c|c|c|c|}
\hline$R$ & $t$ & $L$ & $d_{r}$ & $R / t$ & $L^{*}$ & $d^{*}$ \\
\hline $\mathrm{mm}$ & $\mathrm{mm}$ & $\mathrm{mm}$ & $\mathrm{mm}$ & - & - & - \\
\hline \hline 10000 & 25 & 10000 & 1250 & 400 & 20 & 2,5 \\
\hline 10000 & 10 & 6325 & 790 & 1000 & 20 & 2,5 \\
\hline 10000 & 2 & 2828 & 354 & 5000 & 20 & 2,5 \\
\hline
\end{tabular}

Tabelle 5.2: Variationsparameter der Kreiszylinderschale für unterschiedliche $R / t$-Verhältnisse

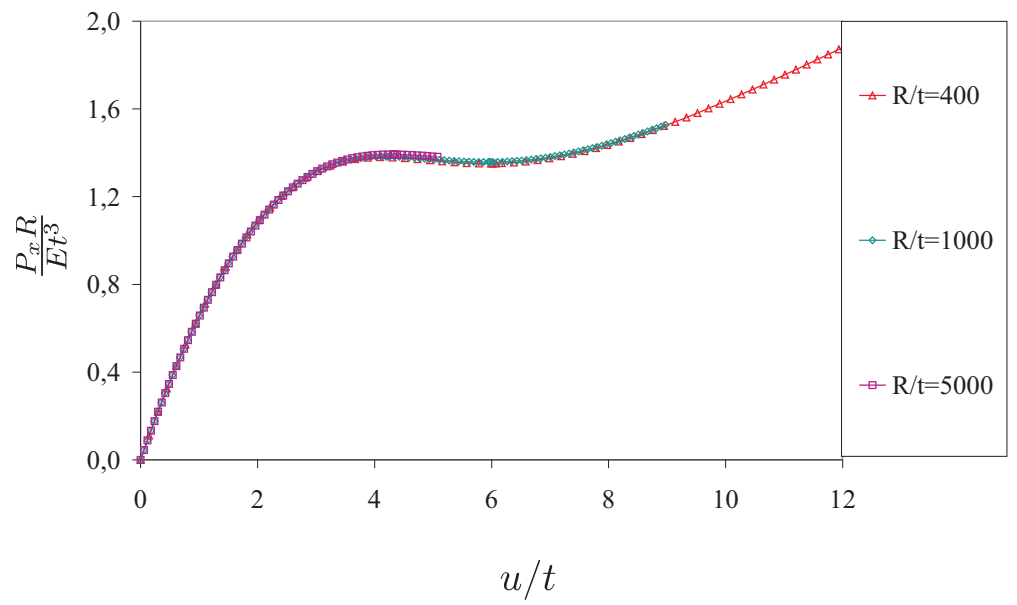

Abbildung 5.2: Last-Verformungskurven von Kreiszylinderschalen unter radialer Einzellast mit $d^{*}=2,5, L^{*}=20$ und $400 \leq R / t \leq 5000$

Die Last-Verformungskurven der untersuchten Kreiszylinderschalen bestätigen die Analogie zur Kugelkappe unter radialer Einzellast insofern als das $R / t$ Verhältnis keinen Einfluss auf das Tragverhalten der Kreiszylinderschale unter radialer Einzellast hat.

\subsection{Einfluss des Stutzens auf das Last- Verformungsverhalten}

Bei Kugelkappen unter radialer Einzellast wurde in Abschnitt 4.3 und in Abschnitt 4.4 gezeigt, dass die Ausführung des Stutzens einen deutlichen Einfluss auf das Last-Verformungsverhalten ausübt. Dieser Einfluss ist auch bei der Kreiszylinderschale zu erwarten. Die relevanten Parameter sind dabei der dimensionslose 
Stutzendurchmesser $d^{*}$, die bezogene Stutzenlänge $l_{r}^{*}$ sowie die Steifigkeit des Stutzens, die durch den E-Modul $E_{r}$ des Werksoffes und die Wanddicke $t_{r}$ gekennzeichnet ist. Zunächst wurde in geometrisch linearen Untersuchungen der Einfluss der Stutzenlänge $l_{r}$ untersucht. Tabelle 5.3 stellt die Abmessungen der Kugelkappe dar. Dabei zeigt sich, dass die Umfangsspannungen der untersuchten Kreiszylinderschalen, die in Abbildung 5.3 über dem Umfang der Kreiszylinderschale aufgetragen sind, von der bezogene Länge $l_{r}^{*}$ des Stutzens abhängen. Je größer $l_{r}^{*}$ ist, desto größer sind auch die Umfangsspannungen am Stutzenanschluss. Die Ursache für diesen Effekt sind die Längsspannungen im Stutzen, die in Abbildung 5.4 entlang des Stutzens aufgetragen sind.

\begin{tabular}{|c|c|c|c|c|c|c|c|}
\hline$R$ & $t$ & $L$ & $d_{r}$ & $t_{r}$ & $l_{r}$ & $d^{*}$ & $l_{r}^{*}$ \\
\hline $\mathrm{mm}$ & $\mathrm{mm}$ & $\mathrm{mm}$ & $\mathrm{mm}$ & $\mathrm{mm}$ & $\mathrm{mm}$ & - & - \\
\hline \hline 10000 & 25 & 10000 & 875 & 25 & 209 & 3,5 & 2 \\
\hline 10000 & 25 & 10000 & 875 & 25 & 1045 & 3,5 & 10 \\
\hline 10000 & 25 & 10000 & 875 & 25 & 5230 & 3,5 & 50 \\
\hline
\end{tabular}

Tabelle 5.3: Variationsparameter der Kreiszylinderschale für unterschiedliche $l_{r}$

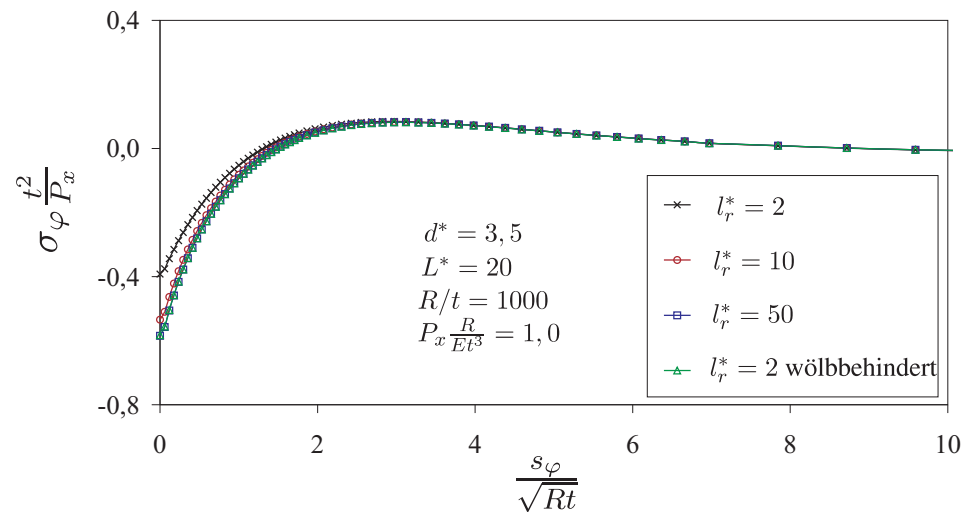

Abbildung 5.3: Umfangsspannungen entlang des Umfangs einer Kreiszylinderschale mit unterschiedlichen bezogenen Stutzenlängen $l_{r}^{*}$

Da sich das freie Ende des Stutzens verwölbt, wirken sich sehr kleine bezogene Stutzenlängen $l_{r}^{*}$ auf die Längsspannungen im Stutzen aus. Dieser Effekt kann eliminiert werden, indem die Verwölbung des freien Randes des Stutzens behindert wird und somit ein unendlich langer Stutzen simuliert wird. Wie die Abbildungen 


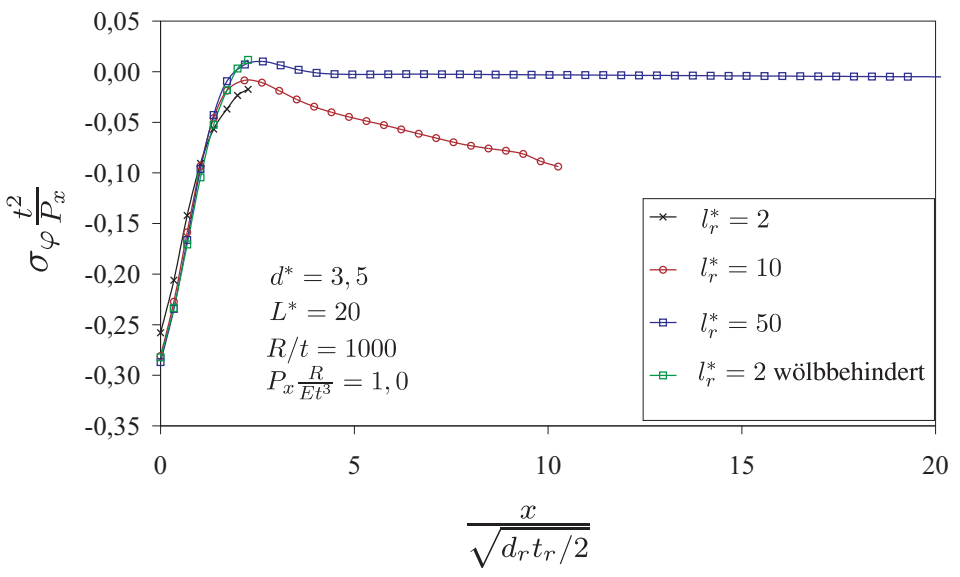

Abbildung 5.4: Längsspannungen entlang des Stutzens einer Kreiszylinderschale mit unterschiedlichen bezogenen Stutzenlängen $l_{r}^{*}$

5.3 und 5.4 zeigen, ist der Spannungsverlauf einer Kreiszylinderschale mit einem sehr kurzen wölbbehinderten Stutzen dem Spannungsverlauf einer Kreiszylinderschale mit einem sehr langen Stutzen identisch.

Im nächsten Schritt wurden Kreiszylinderschalen mit konstantem $L^{*}$ und $R / t$ Verhältnis bei variablem bezogenem Stutzendurchmesser $d^{*}$ und unterschiedlichen Steifigkeiten untersucht. In den numerischen Berechnungen wurde die geometrische Nichtlinearität berücksichtigt. Die Verwölbung der Stutzen am freien Ende wurde dabei behindert. In Abbildung 5.5 sind die Last-Verformungskurven der Kreiszylinderschalen mit den Abmessungen gemäß Tabelle 5.4 aufgetragen. Zudem wurden die Last-Verformungskurven von Kreiszylinderschalen mit einem vollkommen steifen Stutzen $\left(E_{r}=10 E\right)$ und einem sehr nachgiebigen Stutzen $\left(E_{r}=0,1 E\right)$ in Abbildung 5.5 ergänzt. Dabei bestätigt sich die Analogie zum Tragverhalten der Kugelkappe unter radialer Einzellast: Das Tragverhalten der Kreiszylinderschale wird ebenfalls stark vom Stutzendurchmesser und dessen Steifigkeit beeinflusst. Es wurde außerdem eine Kreiszylinderschale untersucht, deren Stutzenende sich frei verwölben konnte und zudem eine kleine bezogene Stutzenlänge von $l_{r}^{*}=5$ hatte. Hier zeigt sich wiederum, dass die Stutzenlänge gerade im nichtlinearen Bereich Einfluss auf das Last-Verformungsverhalten der Kreiszylinderschale hat. 


\begin{tabular}{|c|c|c|c|c|c|c|c|}
\hline$R$ & $t$ & $L$ & $d_{r}$ & $t_{r}$ & $R / t$ & $L^{*}$ & $d^{*}$ \\
\hline $\mathrm{mm}$ & $\mathrm{mm}$ & $\mathrm{mm}$ & $\mathrm{mm}$ & $\mathrm{mm}$ & - & - & - \\
\hline \hline 10000 & 25 & 10000 & 375 & 25 & 400 & 20 & 1,5 \\
\hline 10000 & 25 & 10000 & 625 & 25 & 400 & 20 & 2,5 \\
\hline 10000 & 25 & 10000 & 875 & 25 & 400 & 20 & 3,5 \\
\hline
\end{tabular}

Tabelle 5.4: Variationsparameter der Kreiszylinderschale für unterschiedliche $d^{*}$

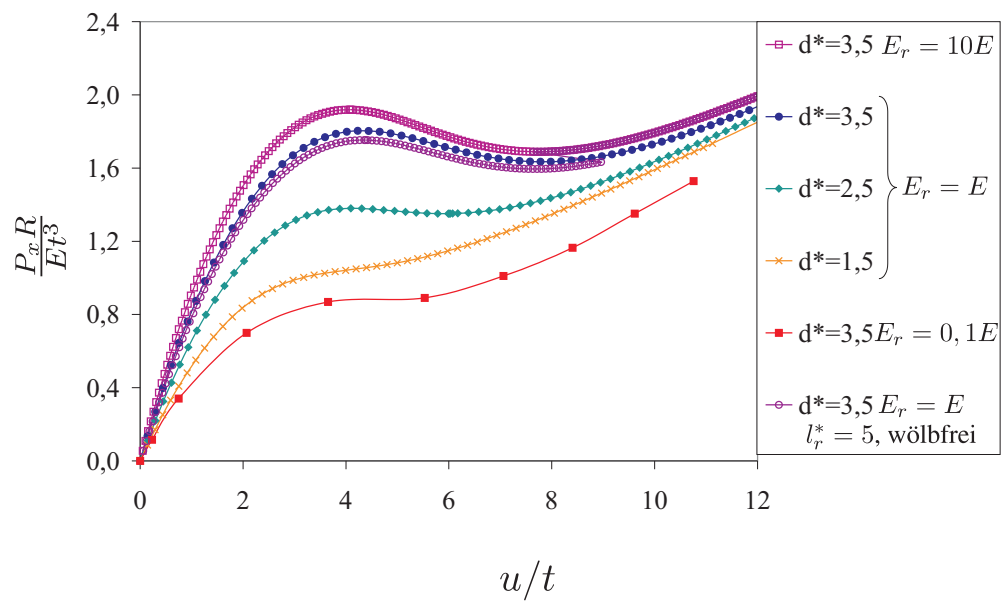

Abbildung 5.5: Last-Verformungskurven einer Kreiszylinderschale unter radialer Einzellast mit $R / t=400, L^{*}=20$ und $1,5 \leq d^{*} \leq 3,5$ mit unterschiedlichen Stutzensteifigkeiten

\subsection{Einfluss des Abstandes der Lastangriffs- punkte in Umfangsrichtung auf das Last- Verformungsverhalten}

Um FE-Modelle mit möglichst geringer Elementanzahl zu erzeugen, ist die Ausnutzung der Symmetriebedingungen ein äußerst effizientes Mittel. Im Fall der Kreiszylinderschale unter radialer Einzellast hängt die Anzahl der Symmetrieebenen und folglich die Größe der FE-Modelle von der Anzahl der Lastangriffspunkte ab. Da keine gegenseitige Beeinflussung zwischen den Verformungen und Spannungen jedes Lastangriffspunktes bestehen soll, ergibt sich die maximale Anzahl der Lastangriffspunkte aus dem Abstand der Punkte zueinander. 
Es wurden numerische Berechnungen ohne Berücksichtigung der geometrischen Nichtlinearität an Kreiszylinderschalen mit zwei Lastangriffpunkten mit einem Abstand von $180^{\circ}$ zueinander durchgeführt. Dabei wurde das $R / t$-Verhältnis und der bezogene Randabstand $L^{*}$ variiert. Die Abmessungen der untersuchten Kreiszylinderschalen sind in Tabelle 5.5 aufgeführt.

\begin{tabular}{|c|c|c|c|c|c|c|}
\hline$R$ & $t$ & $R / t$ & $L$ & $L^{*}$ & $d_{r}$ & $t_{r}$ \\
\hline $\mathrm{mm}$ & $\mathrm{mm}$ & & $\mathrm{mm}$ & - & $\mathrm{mm}$ & $\mathrm{mm}$ \\
\hline \hline 10000 & 25 & 400 & 10000 & 20 & 500 & 25 \\
\hline 10000 & 10 & 1000 & 10000 & 31,6 & 500 & 25 \\
\hline 10000 & 2 & 5000 & 10000 & 70,7 & 500 & 25 \\
\hline
\end{tabular}

Tabelle 5.5: Abmessungen der Kreiszylinderschalen mit zwei Lastangriffspunkten bei unterschiedlichen $R / t$-Verhältnissen

Die mit der Wanddicke $t$ normierten radialen Verschiebungen $u$ sind in Abbildung 5.6 für diese Kreiszylinderschalen unter einer Einheitslast von $P_{x} \frac{R}{E t^{3}}=1$ entlang der Umfangs aufgetragen. Dabei zeigt sich, dass die Verschiebungen entlang des Umfangs schnell abklingen. Im ungünstigsten Fall $(R / t=400)$ sind die Verschiebungen bei etwa $70^{\circ}$ nahezu abgeklungen. Die Verformung entlang des

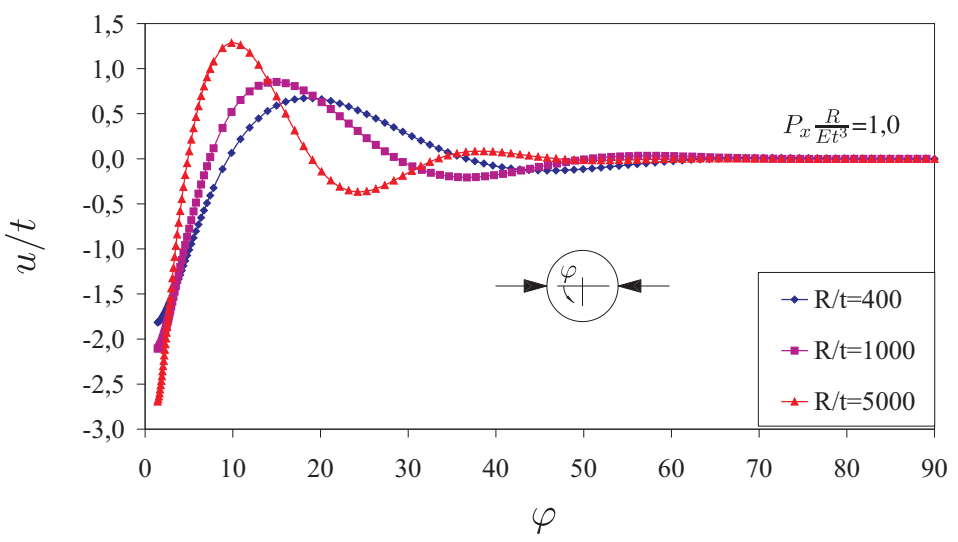

Abbildung 5.6: Radiale Verschiebung $u$ entlang des Umfangs einer Kreiszylinderschale mit unterschiedlichen $R / t$-Verhältnissen

Umfangs einer Kreiszylinderschale mit vier äquidistant entlang des Umfangs angeordneten Lastangriffspunkten ist in Abbildung 5.6 den Verformungen der Kreis- 
zylinderschale mit zwei Lastangriffspunkten gegenübergestellt. Das $R / t$-Verhältnis der Kreiszylinderschalen beträgt 400. Es zeigt sich, dass die Verschiebungen bei $45^{\circ}$ noch nicht vollkommen abgeklungen sind, so dass es zu einer gegenseitigen Beeinflussung der Verschiebungen kommt. Im dargestellten ungünstigsten Fall sind die Verschiebungen der Kreiszylinderschalen mit vier Lasteinleitungspunkten im Abstand von etwa $20^{\circ}$ von der Lasteinleitungsstelle geringfügig größer als die Verschiebungen der Kreiszylinderschalen mit zwei Lasteinleitungspunkten. Da die Verformungen bereits bei $90^{\circ}$ vollständig abgeklungen sind, sind für $0 \leq \varphi \leq 90^{\circ}$ die Verformungen einer Kreiszylinderschale mit nur einem Lastangriffpunkt und mit zwei Lastangriffspunkten identisch.

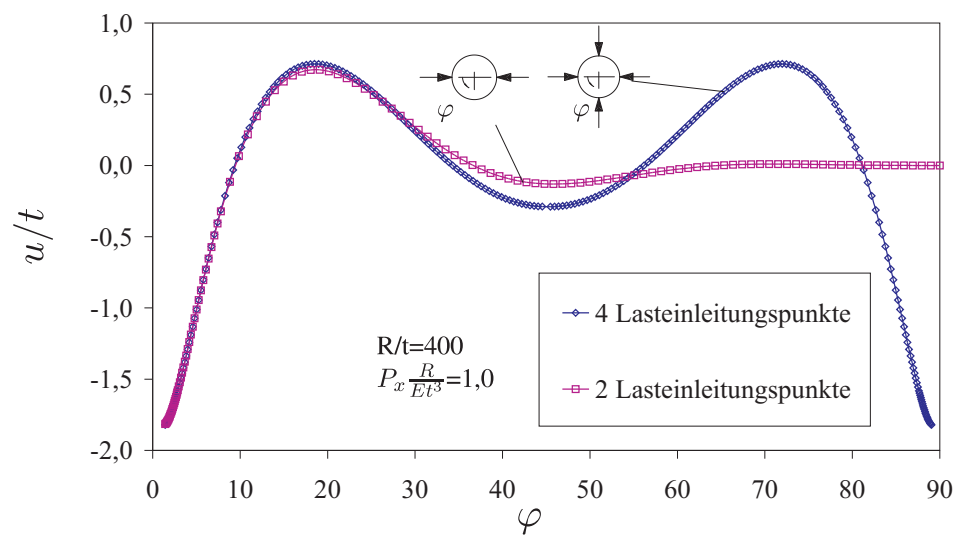

Abbildung 5.7: Radiale Verschiebung $u$ entlang des Umfangs von Kreiszylinderschalen mit zwei und vier Lastangriffspunkten

\subsection{Abklinglänge der Biegestörung}

Die Kreiszylinderschale erfährt durch die radiale Einzellast eine Biegestörung, die zu sehr großen Spannungen und Verformungen am Lastangriffpunkt führt. Diese Biegestörung kling jedoch in Umfangs- und Längsrichtung schnell ab. Aufgrund der Spannungsspitzen am Stutzenanschluss muss dieser Bereich im Modell der Kreiszylinderschale und des Stutzens sehr fein vernetzt werden. Da der hohe Vernetzungsgrad im Stutzenanschlussbereich nicht für die gesamte Kreiszylinderschale beibehalten werden muss, wird im folgenden die Abklinglänge dieser Bie- 
gestörung untersucht, um somit den fein vernetzten vom grob vernetzten Bereich abzugrenzen. Dazu wurden die Krümmungen einer lokal beanspruchten Kreiszylinderschale betrachtet. In numerischen Berechnungen ohne Berücksichtigung geometrisch nichtlinearer Effekte wurden Kreiszylinderschalen mit unterschiedlichen bezogenen Randabständen $L^{*}$ der Lasteinleitungsstelle zum Schalenrand und unterschiedlichen $R / t$-Verhältnissen mit einem konstanten bezogenen Stutzendurchmesser $d^{*}$ von 3,5 untersucht. Die Abmessungen dieser Kreiszylinderschalen sind in Tabelle 5.7 aufgeführt. Die Krümmungen $\kappa$ der verformten Kreiszylinderschalen sind in Abbildung 5.8 entlang des Umfangs und in Abbildung 5.9 entlang des Meridians aufgetragen. Die Umfangs- und Längsachse wurde dabei mit $\sqrt{R t}$ normiert. Die Abmessungen der betrachteten Kreiszylinderschalen sind in Tabelle 5.6 aufgeführt.

\begin{tabular}{|c|c|c|c|c|c|}
\hline$R$ & $t$ & $R / t$ & $L$ & $L^{*}$ & $d^{*}$ \\
\hline $\mathrm{mm}$ & $\mathrm{mm}$ & & $\mathrm{mm}$ & & \\
\hline \hline 10000 & 10 & 1000 & 6325 & 20 & 3,5 \\
\hline 10000 & 10 & 1000 & 12649 & 40 & 3,5 \\
\hline 10000 & 10 & 1000 & 25300 & 80 & 3,5 \\
\hline 10000 & 2,5 & 5000 & 18675 & 40 & 3,5 \\
\hline
\end{tabular}

Tabelle 5.6: Abmessungen der Kreiszylinderschalen mit unterschiedlichen $L^{*}$

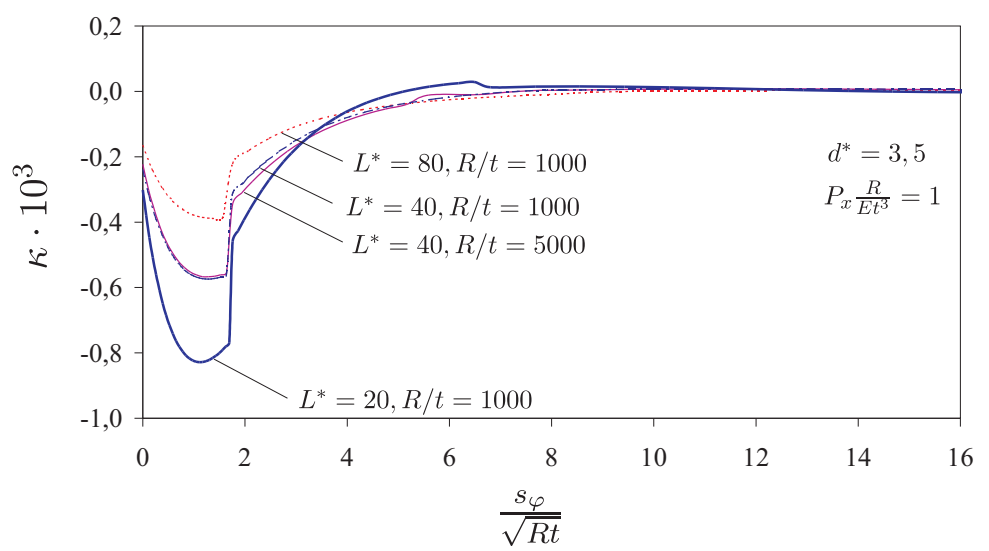

Abbildung 5.8: Krümmung entlang des Umfangs der verformten Kreiszylinderschalen mit unterschiedlichen $L^{*}$ und $R / t$ 


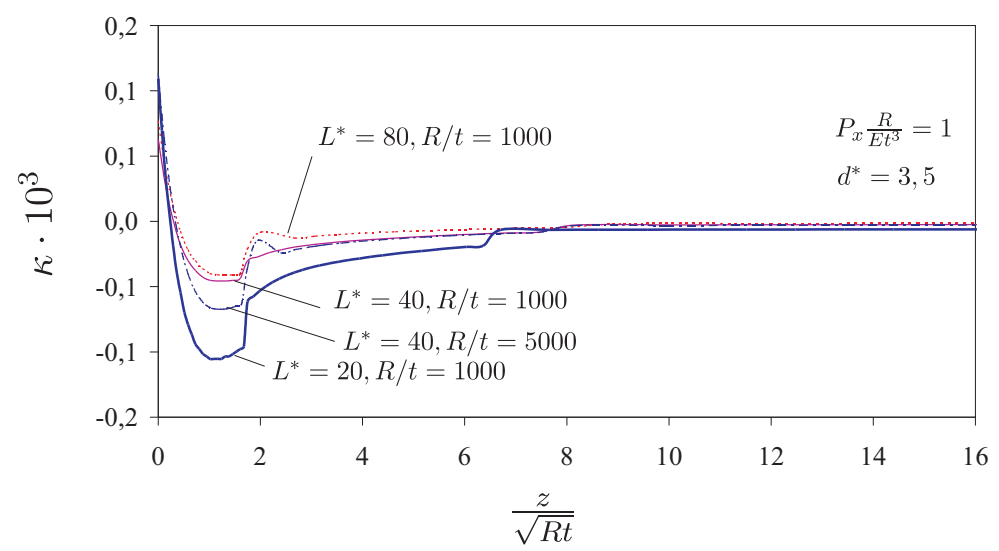

Abbildung 5.9: Krümmung entlang des Meridians der verformten Kreiszylinderschalen mit unterschiedlichen $L^{*}$ und $R / t$

Wie diese beiden Darstellungen zeigen, sind die großen Krümmungen infolge der radialen Einzellast $P_{x}$ (Biegestörung) auf einen kleinen Bereich beschränkt. Dabei ist die Länge dieses Bereiches unabhängig von $L^{*}$ und $R / t$. Sowohl in Umfangs- als auch in Längsrichtung sind die Krümmungen nach einer Länge von etwa $5 \sqrt{R t}$ bereits stark abgeklungen. Dies geht einher mit einer Periode einer gedämpften Schwingung, die bei Kreiszylinderschalen aus Stahl mit einem EModul von $210000 \mathrm{~N} / \mathrm{mm}^{2}$ und einer Querkontraktionszahl von $\mu=0,3$ bei $4,89 \sqrt{R t}$ liegt (vgl. z.B. [56]).

\subsection{Einfluss der Randbedingungen auf das Last- Verformungsverhalten}

Der Einfluss der Lagerung der Schalenränder auf das Tragverhalten der Kreiszylinderschale wurde an Kreiszylinderschalen mit unterschiedlichen bezogenen Randabständen $L^{*}$ für unterschiedliche Randbedingungen untersucht. Dabei wurden eingespannte (RB1), gelenkige (RB2) und freie Ränder (RB3) (vgl. Abschnitt 3.4) betrachtet. In den numerischen Berechnungen wurden keine geometrischen Nichtlinearitäten berücksichtigt. Die Abmessungen der Kreiszylinderschalen sind in Tabelle 5.7 aufgeführt. 


\begin{tabular}{|c|c|c|c|c|c|}
\hline$R$ & $t$ & $R / t$ & $L$ & $L^{*}$ & $d^{*}$ \\
\hline $\mathrm{mm}$ & $\mathrm{mm}$ & & $\mathrm{mm}$ & & \\
\hline \hline 10000 & 10 & 1000 & 1581 & 5 & 3,5 \\
\hline 10000 & 10 & 1000 & 6325 & 20 & 3,5 \\
\hline 10000 & 10 & 1000 & 12649 & 40 & 3,5 \\
\hline 10000 & 10 & 1000 & 25300 & 80 & 3,5 \\
\hline
\end{tabular}

Tabelle 5.7: Abmessungen der Kreiszylinderschalen mit unterschiedlichen $L^{*}$

In Abbildung 5.10 wurden die mit der Wanddicke $t$ normierten radialen Verschiebungen $u$ über der mit $\sqrt{R t}$ normierten Koordinate $z$ unter einer Einheitslast von $P \frac{R}{E t^{3}}=1$ aufgetragen. Trotz unterschiedlicher Randbedingungen unterscheiden sich die Verschiebungen aller untersuchten Kreiszylinderschalen nur geringfügig. Die maximale Abweichung der Maximalwerte liegt für eine Kreiszylinderschale mit $L^{*}=80$ bei etwa $3 \%$.

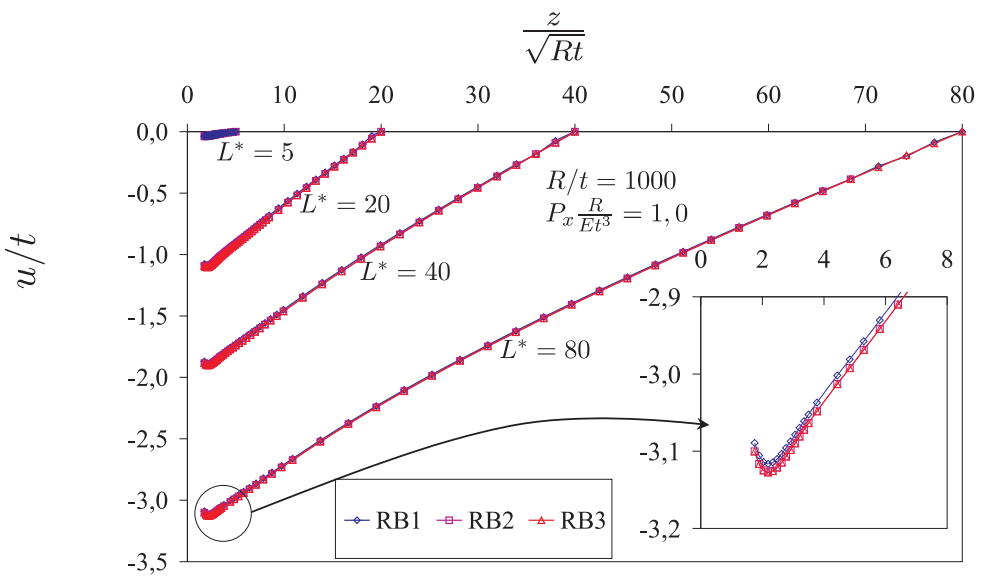

Abbildung 5.10: Radiale Verschiebung entlang des Meridians der Kreiszylinderschale mit unterschiedlichen $L^{*}$ für unterschiedliche Randbedingungen

Die in Tabelle 5.7 aufgeführte Kreiszylinderschale mit $R / t=1000, L^{*}=20$ und $d^{*}=3,5$ wurde in FE-Berechnungen unter Berücksichtigung der geometrischen Nichtlinearitäten untersucht. Die Last-Verformungskurven dieser Kreiszylinderschalen sind in Abbildung 5.11 dargestellt. Dabei zeigt sich, dass auch bei Berück- 
sichtigung großer Verformungen unterschiedliche Randbedingungen keinen wesentlichen Einfluss auf das Lastverformungsverhalten der Kreiszylinderschalen haben.

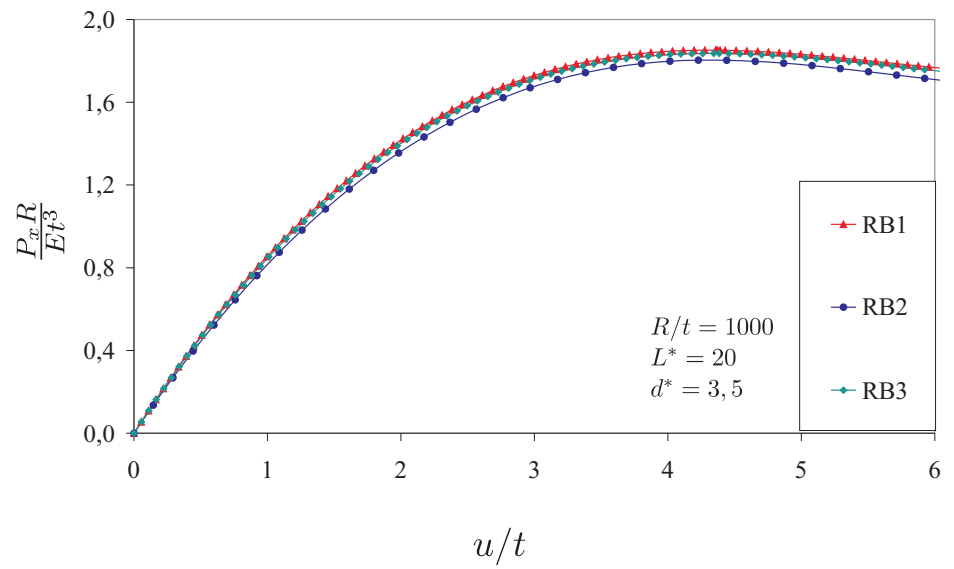

Abbildung 5.11: Last-Verformungskurve einer Kreiszylinderschale mit $R / t=1000, L^{*}=80$ und $d^{*}=3,5$ für unterschiedliche Randbedingungen

\subsection{Interaktion von Innendruck und lokaler Bela- stung}

Die betrachteten Tankbauwerke sind in der Regel mit einem flüssigen oder gasförmigen Medium gefüllt. Der aus dem Füllmedium resultierende Radialdruck erzeugt Membranzugspannungen in der Wand der Kreiszylinderschale, die sich bei zusätzlicher lokaler Belastung mit den daraus entstehenden Spannungen überlagern. An der in Tabelle 5.8 aufgeführten Kreiszylinderschale wurde der Einfluss des Innendrucks auf das Tragverhalten exemplarisch untersucht. Zunächst wurde die Kreiszylinderschale mit einem Innendruck $p_{i}$ von $0,01 \mathrm{~N} / \mathrm{mm}^{2}$ belastet. Anschließend wurden die radialen Einzellasten $P_{x}$ aufgebracht. In Abbildung 5.12 ist die Last-Verformungskurve dieser Kreiszylinderschale dargestellt. Außerdem wurde dort die Last-Verformungskurve aufgetragen, wenn die Kreiszylinderschale nur mit radialen Einzellasten $P_{x}$ belastet wird.

Es zeigt sich, dass sich der Innendruck stabilisierend auf das Tragverhalten der Kreiszylinderschalen auswirkt. Die infolge der lokalen Belastung enstehenden 


\begin{tabular}{|c|c|c|c|}
\hline$R / t$ & $L^{*}$ & $d^{*}$ & $p_{i}$ \\
\hline \hline 400 & 20 & 2,5 & $0,01 \mathrm{~N} / \mathrm{mm}^{2}$ \\
\hline 400 & 20 & 2,5 & - \\
\hline
\end{tabular}

Tabelle 5.8: Abmessungen der Kreiszylinderschale unter radialer Einzellast $P_{x}$ und Innendruck $p_{i}$

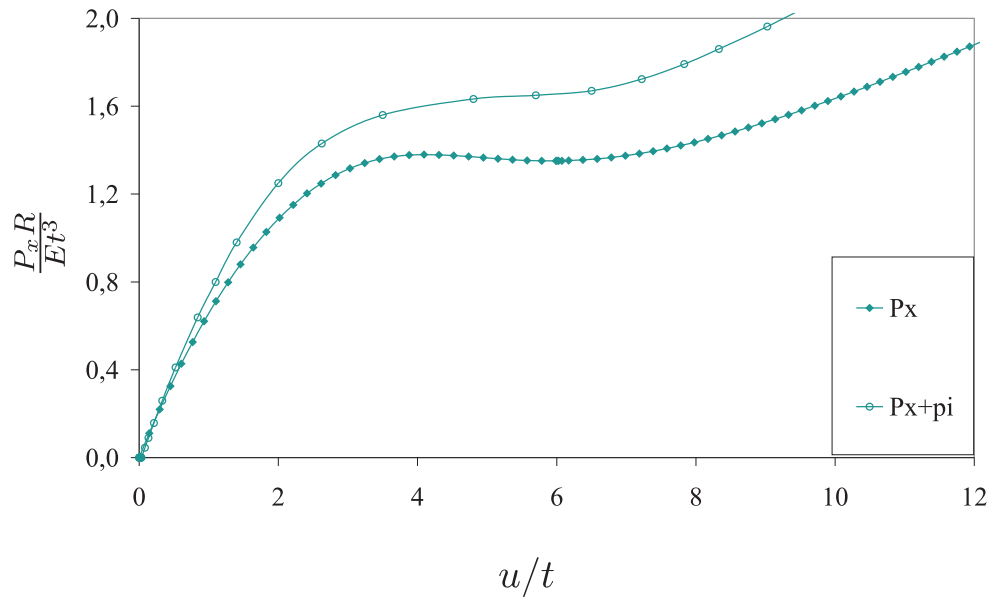

Abbildung 5.12: Last-Verformungskurve einer Kreiszylinderschale mit $R / t=400, L^{*}=20$ und $d^{*}=1,5$ unter Innendruck $p_{i}=0,01 \mathrm{~N} / \mathrm{mm}^{2}$ und radialer Einzellast $P_{x}$ sowie nur unter radialer Einzellast $P_{x}$

Membrandruckspannungen, die zu einem Stabilitätsproblem führen können, werden durch die Membranzugspannungen aus dem Radialdruck reduziert.

\subsection{Vergleich der numerischen Ergebnisse mit ana- lytischen und numerischen Ergebnissen aus WRCB 297}

Im „Welding research council bulletin (WRCB) 297“ [29] wurden Kreiszylinderschalen unter radialer Einzellast und Momentenbelastung in Umfangs- und Meridianrichtung mit der FE-Methode untersucht und mit Ergebnissen verglichen, die sich mit der in [29] aufgeführten analytischen Methode ergeben. In diesem Abschnitt werden die Ergebnisse der Kreiszylinderschalen R500 und R2000 aus 
[29] mit eigenen numerischen Ergebnissen verglichen. Die Vergleichsberechnungen beschränken sich auf den Lastfall radiale Einzellast $P_{x}$. Die Abmessungen der untersuchten Kreiszylinderschalen sowie die dimensionslosen Parameter sind in Tabelle 5.9 und Tabelle 5.10 zusammengestellt.

\begin{tabular}{|c||c|c|c|c|c|}
\hline & $R$ & $t$ & $d_{r}$ & $t_{r}$ & $L$ \\
\hline & $\mathrm{mm}$ & $\mathrm{mm}$ & $\mathrm{mm}$ & $\mathrm{mm}$ & $\mathrm{mm}$ \\
\hline \hline 500R & 12700 & 10,16 & 254 & 5,08 & 36957 \\
\hline 1000R & 25400 & 5,08 & 254 & 5,08 & 36957 \\
\hline
\end{tabular}

Tabelle 5.9: Abmessungen der Kreiszylinderschalen aus [29] und [36]

\begin{tabular}{|c||c|c|c|}
\hline & $\mathrm{R} / \mathrm{t}$ & $d^{*}$ & $L^{*}$ \\
\hline \hline 500R & 1250 & 0,71 & 103 \\
\hline $1000 \mathrm{R}$ & 5000 & 0,71 & 103 \\
\hline
\end{tabular}

Tabelle 5.10: Dimensionslose Parameter der Kreiszylinderschalen aus [29] und [36]

Alle untersuchten Kreiszylinderschalen waren an den oberen und unteren Rändern gelenkig gelagert (RB2). In den eigenen numerischen Berechnungen wurde das in Abschnitt 3.4 beschriebene FE-Modell verwendet. Dabei wurden geometrische und materielle Nichtlinearitäten nicht berücksichtigt. Die maximale Elementlänge im Stutzenanschlussbereich und im Stutzen beträgt in den eigenen FEBerechnungen sowie in den Untersuchungen in [29] maximal 0, 25 $\sqrt{R t}$.

Die Ergebnisse der eigenen FE-Berechnungen und der in [29] dokumentierten FEBerechnungen unter einer Einheitslast von $P_{x} \frac{R}{E t^{3}}=1$ sind in den Tabellen 5.11 bis 5.15 den analytischen Ergebnissen nach WRCB 297 gegenübergestellt. Die dimensionslosen Spannungen in Längs- und Umfangrichtung wurden im Schnittpunkt des Stutzens mit der Längsachse (Stelle A) und dem Umfang (Stelle B) (vgl. Abbildung 3.6) jeweils an der Außen- und Innenseite der Kreiszylinderschale ermittelt. 


\begin{tabular}{|c|c||c|c|c|}
\hline & & $\begin{array}{c}\text { Eigene FE- } \\
\text { Berechnung }\end{array}$ & $\begin{array}{c}\text { FE-Berechnung } \\
\text { in [29] }\end{array}$ & $\begin{array}{c}\text { Analytische } \\
\text { Lösung in [29] }\end{array}$ \\
\hline \hline 500R & außen & $-0,69$ & $-0,68$ & $-1,43$ \\
\hline 500R & innen & 0,56 & 0,52 & 1,20 \\
\hline 1000R & außen & $-0,69$ & $-0,70$ & $-1,91$ \\
\hline 1000R & innen & 0,58 & 0,58 & 1,73 \\
\hline
\end{tabular}

Tabelle 5.11: Dimensionslose Längsspannungen $\sigma_{z} \frac{t^{2}}{P_{x}}$ an der Stelle A

\begin{tabular}{|c|c||c|c|c|}
\hline & & $\begin{array}{c}\text { Eigene FE- } \\
\text { Berechnung }\end{array}$ & $\begin{array}{c}\text { FE-Berechnung } \\
\text { in [29] }\end{array}$ & $\begin{array}{c}\text { Analytische } \\
\text { Lösung in [29] }\end{array}$ \\
\hline \hline 500R & außen & $-1,16$ & $-1,14$ & $-1,08$ \\
\hline 500R & innen & 0,71 & 0,69 & 0,53 \\
\hline 1000R & außen & $-0,89$ & $-0,87$ & $-0,88$ \\
\hline 1000R & innen & 0,46 & 0,45 & 0,37 \\
\hline
\end{tabular}

Tabelle 5.12: Dimensionslose Längsspannungen $\sigma_{z} \frac{t^{2}}{P_{x}}$ an der Stelle B

Es zeigt sich, dass die Ergebnisse aus den eigenen FE-Berechnungen sehr gut mit den in WRCB 297 dokumentierten FE-Berechnungen übereinstimmen. Die numerisch ermittelten Spannungen an der Außenseite sind stets größer als an der Innenseite der Kreiszylinderschale. Dabei sind Längs- und Umfangsspannungen an der Stelle B größer als an der Stelle A, wobei die maximalen Spannungen stets die Umfangsspannungen sind. Bei der Spannungungsermittlung mit der analytischen Methode nach WRCB 297 wird Rotationssymmetrie zur Stutzenlängsachse angenommen. Dies bedeutet, dass an der Stelle A die in WRCB 297 mit $\sigma_{r}$ bezeichneten Spannungen die Längsspannungen $\sigma_{z}$ und die mit $\sigma_{\theta}$ bezeichneten Spannungen die Umfangsspannungen $\sigma_{\varphi}$ sind. An der Stelle B sind dagegen $\sigma_{r}$ die Umfangsspannungen und $\sigma_{\theta}$ die Längsspannungen. Aus diesem Grund werden die numerisch ermittelten Längs- und Umfangsspannungen durch die analytisch ermittelten Werte an der Stelle A weit überschätzt. Wie die Gegenüberstellung der Längsspannungen an der Stelle B in Tabelle 5.12 zeigt, stimmen die Ergebnisse recht gut überein, wobei die analytisch bestimmten Spannungen der Kreiszylinderschale R500 leicht auf der unsicheren Seite liegen. Die Umfangsspannungen im Punkt B werden mit der analytischen Methode dagegen um bis zu $20 \%$ überschätzt.

Die analytisch ermittelten dimensionslosen Verschiebung $u / t$ unter dem Stutzen stimmen gut mit den numerisch ermittelten Verschiebungen überein. 


\begin{tabular}{|c|c||c|c|c|}
\hline & & $\begin{array}{c}\text { Eigene FE- } \\
\text { Berechnung }\end{array}$ & $\begin{array}{c}\text { FE-Berechnung } \\
\text { in [29] }\end{array}$ & $\begin{array}{c}\text { Analytische } \\
\text { Lösung in [29] }\end{array}$ \\
\hline \hline 500R & außen & $-0,79$ & $-0,79$ & $-1,08$ \\
\hline 500R & innen & 0,22 & 0,26 & 0,53 \\
\hline 1000R & außen & $-0,45$ & $-0,48$ & $-0,88$ \\
\hline 1000R & innen & 0,01 & 0,01 & 0,37 \\
\hline
\end{tabular}

Tabelle 5.13: Dimensionslose Umfangsspannungen $\sigma_{\varphi} \frac{t^{2}}{P_{x}}$ an der Stelle A

\begin{tabular}{|c|c||c|c|c|}
\hline & & $\begin{array}{c}\text { Eigene FE- } \\
\text { Berechnung }\end{array}$ & $\begin{array}{c}\text { FE-Berechnung } \\
\text { in [29] }\end{array}$ & $\begin{array}{c}\text { Analytische } \\
\text { Lösung in [29] }\end{array}$ \\
\hline \hline 500R & außen & $-1,22$ & $-1,19$ & $-1,43$ \\
\hline 500R & innen & 1,02 & 0,98 & 1,20 \\
\hline 1000R & außen & $-1,76$ & $-1,77$ & $-1,91$ \\
\hline 1000R & innen & 1,58 & 1,59 & 1,73 \\
\hline
\end{tabular}

Tabelle 5.14: Dimensionslose Umfangsspannungen $\sigma_{\varphi} \frac{t^{2}}{P_{x}}$ an der Stelle B

\begin{tabular}{|c|c||c|c|}
\hline & $\begin{array}{c}\text { Eigene FE- } \\
\text { Berechnung }\end{array}$ & $\begin{array}{c}\text { FE-Berechnung } \\
\text { in [29] }\end{array}$ & $\begin{array}{c}\text { Analytische } \\
\text { Lösung in [29] }\end{array}$ \\
\hline \hline 500R & $-4,73$ & $-4,68$ & $-4,68$ \\
\hline 1000R & $-4,53$ & $-4,47$ & $-4,69$ \\
\hline
\end{tabular}

Tabelle 5.15: Dimensionslose Durchiegung $u / t$ 


\subsection{Vergleich der numerischen Ergebnisse mit Ver- suchsergebnissen der ,Chicago bridge and iron company" und analytischen Ergebnissen aus WRCB 297}

Die Versuchen an Kreiszylinderschalen aus Stahl unter radialer Einzellast sowie Momentenbelastung in Umfangs- und Meridianrichtung der „Chicago bridge and iron company (CBI)“ in [36] sind in [31] ausführlich beschrieben und werden dort auch den mit WRCB 297 analytisch ermittelten Ergebnissen gegenübergestellt. Für die Vergleichsberechnungen mit der FE-Methode wurden die Versuchskörper CBI-1 und CBI-2 mit einem $R / t$-Verhältnis von 524 herangezogen. An den oberen und unteren Rändern waren die Kreiszylinder gelenkig gelagert (RB2). Es waren zwei einander gegenüberliegende Stutzen an die Kreiszylinder angeschweißt. Alle im Versuch gemessenen Spannungen lagen im elastischen Bereich. Die Abmessungen der untersuchten Kreiszylinder sind in Tabelle 5.16 und Tabelle 5.17 aufgeführt.

\begin{tabular}{|c||c|c|c|c|c|}
\hline & $R$ & $t$ & $d_{r}$ & $d_{r}$ & $L$ \\
\hline & $\mathrm{mm}$ & $\mathrm{mm}$ & $\mathrm{mm}$ & $\mathrm{mm}$ & $\mathrm{mm}$ \\
\hline \hline CBI-1 & 3943 & 7,52 & 84 & 4,75 & 1707 \\
\hline CBI-2 & 3943 & 7,52 & 211 & 8,18 & 1707 \\
\hline
\end{tabular}

Tabelle 5.16: Abmessungen der Prüfkörper CBI-1 und CBI-2 [36]

\begin{tabular}{|c||c|c|c|}
\hline & $R / t$ & $d^{*}$ & $L^{*}$ \\
\hline \hline CBI-1 & 524 & 0,49 & 10 \\
\hline CBI-2 & 524 & 1,23 & 10 \\
\hline
\end{tabular}

Tabelle 5.17: Dimensionslose Parameter Prüfkörper CBI-1 und CBI-2 [36]

Für die numerischen Vergleichsberechnungen wurde das in Abschnitt 3.4 beschriebene Modell unter einer Einzellast $P_{x}$ verwendet. Da die Versuchergebnisse im linearen Bereich lagen, traf das angesetzte elastische Werkstoffverhalten zu. Geometrisch nichtlineare Effekte wurden dabei nicht berücksichtigt. In [31] sind die Umfangsspannungen der Kreiszylinderschale CBI-2 unter einer radialen Einzellast in acht Messpunkte entlang des Umfangs dokumentiert. Dort sind auch die Umfangsspannungen aufgeführt, die mit einem Programm zur iterativen Lösung nichtlinearer Gleichungssystem (FAST, vgl. [32]) berechnet wurden und 
dabei von einem vollkommen steifen Stutzen ausgehen. Die Spannungsverläufe aus [31] sowie die Umfangsspannungen aus den FE-Berechnungen sind in Abbildung 5.13 unter einer radialen Stutzenlast $P_{x}$ von $9080 \mathrm{~N}$ entlang des Umfangs aufgetragen.

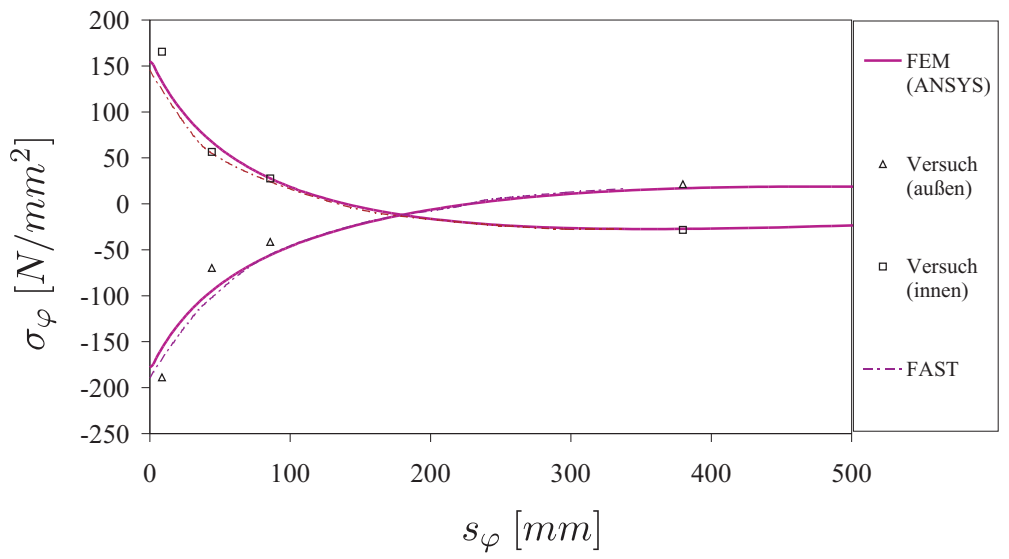

Abbildung 5.13: Umfangsspannungen entlang des Umfangs des Kreiszylinders CBI-2 aus [31] unter einer Last von $9080 \mathrm{~N}$

Wie Abbildung 5.13 deutlich zeigt, stimmen die mit der FE-Methode ermittelten Ergebnisse sehr gut mit den mit Hilfe des Programms FAST ermittelten Ergebnissen überein. Die Spannungen, die am Prüfkörper CBI-2 mehr als etwa $50 \mathrm{~mm}$ vom Stutzenanschluss entfernt gemessen wurden, zeigen ebenfalls eine sehr gute Übereinstimmung mit den numerisch ermittelten Ergebnissen. An den Messpunkten unmittelbar am Stutzenanschluss liegen die gemessenen Spannungen dagegen über den numerisch ermittelten Spannungen. Mit einer Abweichung von maximal 25\% ist die Übereinstimmung der Ergebnisse dennoch zufriedenstellend.

Die Ergebnisse der FE-Berechnungen sowie der Versuche CBI-1 und CBI-2 aus [36] für den Lastfall $P_{x}$ sind in den Tabellen 5.18 bis 5.22 als dimensionslose Größen aufgeführt. Zudem wurden die Längs- und Umfangsspannungen sowie die Verschiebungen mit WRCB 297 [29] für diese beiden Kreiszylinderschalen ermittelt. Da die Dehnmessstreifen, die zur Messung der Spannungen verwendet wurden, nicht unmittelbar am Schnittpunkt des Kreiszylinders mit dem Stutzen apliziert werden konnten, sind in den folgenden Tabellen die mit der FEMethode ermittelten Spannungen auch an den Stellen angegeben, an denen sich 
die Messpunkte befanden. Gemäß den äußerst ungenauen Angaben zu den Positionen der Dehnmesstreifen wurde der Abstand $s_{i}$ des Messpunktes zum Schnittpunkt in Versuch CBI-1 mit $1,4 t_{r}+0,5 t_{r}=9 \mathrm{~mm}$ und in Versuch CBI-2 mit $t_{r}+0,5 t_{r}=12 \mathrm{~mm}$ abgeschätzt.

\begin{tabular}{|c|c||c|c|c|c|}
\hline & \multicolumn{1}{|c||}{} & \multicolumn{2}{c|}{ A+s $s_{i}$} & \multicolumn{2}{c|}{ A } \\
\hline & & FEM & Versuch [36] & FEM & Analyt. [29] \\
\hline \hline CBI-1 & außen & $-0,86$ & $-0,71$ & $-0,99$ & $-1,78$ \\
\hline CBI-1 & innen & 0,59 & 0,61 & 0,75 & 1,46 \\
\hline CBI-2 & außen & $-0,45$ & $-0,66$ & $-0,49$ & $-1,40$ \\
\hline CBI-2 & innen & 0,33 & 0,42 & 0,40 & 1,24 \\
\hline
\end{tabular}

Tabelle 5.18: Dimensionslose Längsspannungen $\sigma_{z} \frac{t^{2}}{P_{x}}$ im Punkt A oder $\mathrm{A}+s_{i}$

\begin{tabular}{|c|c||c|c|c|c|}
\hline & \multicolumn{1}{|c||}{} & \multicolumn{2}{c|}{$\mathrm{B}+s_{i}$} & \multicolumn{2}{c|}{ B } \\
\hline & & FEM & Versuch [36] & FEM & Analyt. [29] \\
\hline \hline CBI-1 & außen & $-0,99$ & $-0,75$ & $-1,04$ & $-1,09$ \\
\hline CBI-1 & innen & 0,67 & 0,45 & 0,75 & 0,59 \\
\hline CBI-2 & außen & 0,52 & $-0,55$ & $-0,53$ & $-0,63$ \\
\hline CBI-2 & innen & 0,23 & 0,41 & 0,23 & 0,27 \\
\hline
\end{tabular}

Tabelle 5.19: Dimensionslose Längsspannungen $\sigma_{z} \frac{t^{2}}{P_{x}}$ im Punkt B oder $\mathrm{B}+s_{i}$

\begin{tabular}{|c|c||c|c|c|c|}
\hline & \multicolumn{1}{|c||}{} & \multicolumn{2}{|c|}{$\mathrm{A}+s_{i}$} & \multicolumn{2}{c|}{ A } \\
\hline & & FEM & Versuch [36] & FEM & Analyt. [29] \\
\hline \hline CBI-1 & außen & $-0,92$ & $-0,43$ & $-0,94$ & $-1,09$ \\
\hline CBI-1 & innen & 0,52 & 0,17 & 0,46 & 0,59 \\
\hline CBI-2 & außen & $-0,32$ & $-0,37$ & $-0,32$ & $-0,63$ \\
\hline CBI-2 & innen & 0,03 & 0,11 & 0,03 & 0,27 \\
\hline
\end{tabular}

Tabelle 5.20: Dimensionslose Umfangsspannungen $\sigma_{\varphi} \frac{t^{2}}{P_{x}}$ im Punkt A oder $\mathrm{A}+s_{i}$

Die numerisch ermittelten Längsspannungen des Kreiszylinders CBI-1 liegen rund 25\% über den gemessenen Längsspannungen, wohingegen die numerisch ermittelten Längsspannungen im Kreiszylinder CBI-2 um bis zu $45 \%$ unterhalb der gemessenen Längsspannungen liegen. Die mit WRCB 297 [29] ermittelten Spannungen werden mit den numerisch ermittelten Spannungen unmittelbar im Schnittpunkt des Stutzens mit der Kreiszylinderschale verglichen. Da bei der analytischen Methode nach WRCB 297 Rotationssymmetrie bezüglich der 


\begin{tabular}{|c|c||c|c|c|c|}
\hline & \multicolumn{1}{|c||}{} & \multicolumn{2}{|c|}{$\mathrm{B}+s_{i}$} & \multicolumn{2}{c|}{ B } \\
\hline & & FEM & Versuch [36] & FEM & Analyt. [29] \\
\hline \hline CBI-1 & außen & $-1,13$ & $-1,30$ & $-1,36$ & $-1,78$ \\
\hline CBI-1 & innen & 0,84 & 1,10 & 1,07 & 1,46 \\
\hline CBI-2 & außen & $-0,93$ & $-1,24$ & $-1,11$ & $-1,40$ \\
\hline CBI-2 & innen & 0,78 & 1,16 & 0,97 & 1,24 \\
\hline
\end{tabular}

Tabelle 5.21: Dimensionslose Umfangsspannungen $\sigma_{\varphi} \frac{t^{2}}{P_{x}}$ im Punkt B oder B $+s_{i}$

\begin{tabular}{|c||c|c|c|}
\hline & FEM & Versuch [36] & Analyt. [29] \\
\hline \hline CBI-1 & $-4,73$ & $-4,68$ & $-4,68$ \\
\hline CBI-2 & $-4,53$ & $-4,47$ & $-4,69$ \\
\hline
\end{tabular}

Tabelle 5.22: Dimensionslose Durchbiegung $u / t$

Stutzenlängsachse angenommen wird, entsprechen die Längsspannungen an der Stelle A den Umfangsspannungen an der Stelle B, sowie die Umfangsspannungen in A den Längsspannungen in B. Aus diesem Grund werden für den Vergleich nur die Spannungen an der Stelle B herangezogen, da an dieser Stelle die FEBerechnung die maximalen Spannungen liefert. Mit der analytischen Methode werden die Längsspannungen in den Kreiszylindern zufriedenstellend und zur sicheren Seite hin abgeschätzt.

Bei den Umfangsspannungen an der Stelle $\mathrm{A}+s_{i}$ des Prüfkörpers CBI-1 zeigen sich große Abweichungen zwischen den numerischen Ergebnissen und den Versuchsergebnissen. Fraglich sind dabei wiederum die Angaben zu den Messpunkten. Dagegen stimmen numerische und experimentell bestimmte Ergebnisse bei der Kreiszylinderschale CBI-2 gut überein. An der Stelle $\mathrm{B}+s_{i}$, wo die Umfangsspannungen stets größer sind als an der Stelle $\mathrm{A}+s_{i}$ liegen die gemessenen Umfangsspannungen zwischen $15 \%$ und $30 \%$ über den numerisch bestimmten Spannungen. Die Spannungen, die sich nach der analytischen Lösung ergeben, überschätzen die Spannungen aus Versuch und FE-Berechnung um rund $50 \%$. Wie Tabelle 5.22 zeigt, stimmen die gemessenen Verschiebungen bei beiden Kreiszylindern sehr gut mit den numerisch und analytisch ermittelten Ergebnissen überein.

Da die Spannungen unmittelbar am Stutzenanschluss sehr groß sind und dann sehr schnell abklingen, führen kleine Abweichungen bei den Positionsangaben der Messpunkte zu sehr großen Abweichungen bei den Spannungen. Zudem ist die Lage der Messpunkte in [36] nur sehr ungenau dokumentiert, so dass die Abweichungen der Spannungen aus den FE-Berechnungen gegenüber den im Versuch gemessenen Spannungen von bis zu $40 \%$ als gute Übereinstimmung zu werten sind. 


\subsection{Zusammenfassung}

Die Untersuchungen an Kreiszylinderschalen unter radialer Einzellast $P_{x}$ haben gezeigt, dass das Tragverhalten der Kreiszylinderschale in vielen Punkten analog zu dem der Kugelkappe ist. Für den Lastfall $P_{x}$ wird das Tragverhalten der Kreiszylinderschale maßgeblich vom bezogenen Randabstand $L^{*}$ und vom bezogenen Stutzenabstand $d^{*}$ beeinflusst. Dabei bestimmt das Verhältnis der Biegeanteile zu den Membrananteilen die Charakteristik der Last-Verformungskurven, wie in Abschnitt 4.3 für die Kugelkappe ausführlich gezeigt und durch Untersuchungen an der Kreiszylinderschale bestätigt wurde. Das $R / t$-Verhältnis wirkt sich dagegen nicht auf den Verlauf der Last-Verformungskurven dieser Strukturen aus. Ein weiterer Einflussfaktor ist die Steifigkeit des Stutzens. Der Stutzen ist annähernd steif, wenn der E-Modul $E_{r}$ des Stutzens gleich dem E-Modul der Kreiszylinderschale ist und die Wanddicke $t_{r}$ des Stutzens mindestens so groß wie die Wanddicke $t$ des Mantelbleches ist. Dabei spielt die Länge des Stutzens keine Rolle, wenn die Verwölbung des freie Endes verhindert wird. Im FE-Modell wird dies erreicht, indem der Stutzen sehr lang ausgeführt $\left(l_{r}^{*}>50\right)$ oder die Verwölbung am freien Ende des Stutzens behindert wird.

Der lokale Charakter der Verformungen und Spannungen der Kugelkappe unter einer radialen Einzellast ist auch bei der Kreiszylinderschale vorhanden. Dies zeigt sich darin, dass die Biegestörung schnell abgeklungen ist.

Der Radialdruck aus dem Füllmedium wirkt sich stabilisierend auf das Tragverhalten der lokal beanspruchten Kreiszylinderschale aus, so dass die Kreiszylinderschale ohne Lagermedium den ungünstigsten Fall abdeckt.

Der Vergleich der Ergebnisse aus experimentellen, analytischen und numerischen Untersuchungen mit eigenen FE-Ergebnissen zeigt, dass die Kreiszylinderschale unter lokaler Belastung mit dem verwendeten FE-Modell zuverlässig abgebildet wird. Die in diesem Kapitel gewonnenen Erkenntnisse führen für weitere numerische Untersuchungen zu folgenden Schlussfolgerungen:

1. Die Lagerung der Schalenränder hat nur einen sehr geringen Einfluss auf das Tragverhalten der Kreiszylinderschale. Vereinfachend und auf der sicheren Seite liegend kann eine gelenkige Lagerung (RB2) der Schalenränder angenommen werden.

2. Aufgrund des Abklingverhaltens der Biegstörung werden Kreiszylinderschalen mit zwei Stutzen, die in Umfangsrichtung einen Abstand von $180^{\circ}$ haben, betrachtet. Dabei wird die Symmetrie ausgenutzt und nur ein Achtel der Kreiszylinderschale abgebildet.

3. Im Lasteinleitungsbereich wird die Kreiszylinderschale sehr fein vernetzt. 
Aufgrund der Spannungsspitzen wird dort die maximale Elementlänge auf $0,25 \sqrt{R t}$ begrenzt. In den Bereichen der Kreiszylinderschale die einen Abstand $\left(d_{n} / 2\right)$ von mindestens $10 \sqrt{R t}$ zur Stutzenachse haben, können die Elemente eine Länge von bis zu $\sqrt{R t}$ haben.

Die Untersuchungen in diesem Abschnitt beschränkten sich auf den Lastfall radiale Einzellast $P_{x}$. Kreiszylinderschalen, die durch Momente in Umfangsrichtung $\left(M_{\varphi}\right)$ oder Meridianrichtung $\left(M_{z}\right)$ beansprucht werden, erfahren eine Biegestörung, deren Abklingverhalten identisch ist mit dem der Kreiszylinderschale unter radialer Einzellast. In Anbetracht dieses Sachverhaltes können die unter 1. bis 3. getroffenen Schlussfolgerungen auch auf Kreiszylinderschalen unter Einwirkung eines Moments in Umfang- oder Meridianrichtung angewandt werden. Die Bedingungen, die bei Erstellung des FE-Modells der Kreiszylinderschale eingehalten werden müssen, sind in Tabelle 5.23 zusammengefasst.

Die Einflüsse der Parameter $L^{*}, d^{*}$ und $R / t$ auf das Tragverhalten treffen dagegen nicht zwangsläufig auf die Lastfälle $M_{z}$ und $M_{\varphi}$ zu und müssen für den jeweiligen Lastfall gesondert untersucht werden.

\begin{tabular}{|c|c|}
\hline Parameter & Bedingung \\
\hline \hline$t_{r} / t$ & $\geq 1,0$ \\
\hline$E_{r} / E$ & $\geq 1,0$ \\
\hline$l_{r}$ & $\geq 50 \sqrt{d_{r} t_{r} / 2}$ \\
\hline$d_{n}$ & $\geq 20 \sqrt{R t}$ \\
\hline Elementlänge & $\leq 0,25 \sqrt{R t}$ \\
\hline Randbedingungen & $u=0 ; v=0$ \\
\hline Anzahl der Stutzen & 2 \\
\hline
\end{tabular}

Tabelle 5.23: Bedingungen für das FE-Modell einer Kreiszylinderschale unter Einwirkung lokaler Lasten 


\section{Numerische Serienberechnungen von Kreiszylinderschalen unter lokaler Belastung}

\subsection{Umfang der Untersuchungen}

Der Umfang der untersuchten Abmessungen orientiert sich an ausgeführten Tankbauwerken und den Mindestwanddicken der Mantelbleche in den gängigen Normen und Regelwerken. Es werden dünnwandige Kreiszylinderschalen im Bereich von $400 \leq R / t \leq 5000$ untersucht. Der bezogene Abstand $L^{*}$ der Lasteinleitungsstelle zum Rand der Kreiszylinderschale resultiert aus den obengenannten $R / t$-Verhältnissen und in der Baupraxis relevanten Schalenlängen $H$. Daraus ergibt sich für $L^{*}$ ein Parameterumfang zwischen 10 und 80. Aus den Abmessungsbereichen für Radius und Wanddicke ergibt sich für den Stutzen ein Parameterbereich von $0,5 \leq d^{*} \leq 3,5$. Es werden Baustähle mit linearelastischidealplastischem Werkstoffgesetz nach Abschnitt 3.5 untersucht, deren Streckgrenzen $f_{y}$ zwischen $240 \mathrm{~N} / \mathrm{mm}^{2}$ und $690 \mathrm{~N} / \mathrm{mm}^{2}$ liegen. Im Rahmen der Serienberechnungen werden die Lastfälle radiale Einzellast $P_{x}$, Momente in Umfangsrichtung $M_{\varphi}$ und Moment in Meridianrichtung $M_{z}$ untersucht.

\subsection{Berechnungskonzepte}

Die numerischen Untersuchungen werden in Abhängigkeit von den berücksichtigten Nichtlinearitäten nach den Berechnungskonzepten in DIN V ENV 1993-16:2005-05 benannt. Diese sind:

- Lineare elastische Berechnung (LA): Den Berechungen liegt die Annahme eines linear elastischen Werkstoffverhaltens und kleiner Verformungen zugrunde.

- Geometrisch nichtlineare elastische Berechnung (GNA): In den Berechnungen wird von einem elastischen Wekstoffverhalten ausgegangen. Es werden 
große Verformungen berücksichtigt, und das Gleichgewicht wird am verformten System gebildet.

- Materiell nichtlineare Berechnung (MNA): In den Berechnungen wird von kleinen Verformungen und einem elasto-plastischen Wekstoffgesetz ausgegangen.

- Geometrisch und materiell nichtlineare Berechung (GMNA): Den Berechnungen liegt die Annahme eines elasto-plastischen Wekstoffgesetzes und großer Verformungen zugrunde.

Laut DIN V ENV 1993-1-6:2005-05 sind zusätzlich geometrische Imperfektionen in GNIA und GMNIA Berechnungen zu berücksichtigen. Diese geometrische Imperfektion wird in den Berechnungen als Formabweichung von der idealen Schalenwand angesetzt, deren Größenordnung im Bereich der Schalenwanddicke $t$ liegt. Die Verformungen, die durch die lokale Belastung senkrecht zur Schalenwand hervorgerufen werden, können dagegen selbst als Imperfektion betrachtet werden und sind zudem ein Vielfaches größer als die gemäß Regelwerk anzusetzenden Imperfektionen. Daher spielen diese Imperfektionen für die betrachteten Lastfälle eine untergeordnete Rolle und es werden in den folgenden Berechnungen keine zusätzlichen geometrischen Imperfektionen angesetzt.

\subsection{Definition der kritischen Last bei geometrisch nichtlinearen Berechnungen}

Die Charakteristik der Last-Verformungskurven kann anschaulich am Beispiel der radialen Einzellast in Abhängigkeit vom bezogenen Stutzendurchmesser $d^{*}$ anhand der Last-Verformungskurven in Abbildung 6.1 erläutert werden. Für große bezogene Stutzendurchmesser $d^{*}$, die oberhalb eines Grenzwertes $d_{g r}^{*}$ liegen, hat die Last-Verformungskurve einen linearen Anfangsbereich mit einer Anfangssteifigkeit $c_{i}^{*}$, die umso größer ist, je größer $d^{*}$ ist. Unter großer Zunahme der Verformungen wird die Kurve nichtlinear, bis schließlich ein lokales Maximum auftritt. Nach Erreichen des Maximums fällt die Last ab und steigt schließlich wieder an. In diesem Fall liegt ein Stabilitätsproblem vor, wobei das lokale Maximum die kritische Last $S_{i, c r i t, k}^{*}$ im Sinne einer Durchschlagslast nach Abschnitt 2.1 ist. Der Index $i$ steht in diesem Fall für den jeweiligen Lastfall, der Index $k$ gibt die Berechnungsart an.

Für kleine bezogene Stutzendurchmesser, d.h. wenn $d^{*}<d_{g r}^{*}$ ist, verläuft die Last-Verformungskurve zunächst linear. Die Steigung $c_{i}^{*}$ im linearen Bereich ist dabei ebenfalls abhängig von $d^{*}$. Unter großer Verformungszunahme 
wird die Kurve flacher und steigt nach einem Krümmungswechsel erneut steil an. Diese Kurven sind stets monton steigend, was darauf hindeutet, dass kein Stabilitätsproblem auftritt und die Bestimmung einer Grenzlast ein Spannungsproblem ist. In diesen Fällen erfolgt die Bestimmung der Grenzlasten indirekt über die normierten Last-Verformungskurven. Dazu werden zunächst die LastVerformungskurven mit einem eindeutigen lokalen Maximum $\left(d^{*}>d_{g r}^{*}\right)$ mit der kritischen Last $S_{i, c r i t, k}$ normiert. Dadurch fallen die Last-Verformungskurven im Vorbeulbereich annähernd zusammen (Abbildung 6.1(b)). Die Last, mit der eine Last-Verformungskurve ohne lokales Maximum $\left(d^{*}<d_{g r}^{*}\right)$ in diese Einheitskurve überführt werden kann, ist die gesuchte Grenzlast. Unabhängig davon, ob ein Stabilitäts- oder Spannungsproblem vorliegt, wird im Folgenden diese Grenzlast als kritische Last $S_{i, c r i t, k}$ bezeichnet. Diese Bestimmung der kritischen Lastgröße wurde gewählt, da sie auf der Charakterisitik der Lastverformungskurve basiert und daher einen Zusammenhang zwischen dem verformungskritischen Zustand beim Auftreten eines Stabilitätsproblems und dem entprechenden Zustand nicht stabilitätsgefährdeter Struktutren herstellt. Das Abgrenzungskriterium ist eine frei gewählte verformungskritische Lastgröße, die die kritische Last zur sicheren Seite hin abschätzt.

Bereits beim Auftreten des lokalen Maximums betragen die maximalen Verformungen ein Vielfaches der Wanddicke. Aufgrund dieser großen Verformungen kommt dem Nachbeulbereich eine untergeordnete Bedeutung zu, er wird im Rahmen dieser Arbeit nicht betrachtet. Da die Bezeichnung Vorbeulbereich nur im Zusammenhang mit einem Stabilitätsproblem zutreffend ist, wird der betrachtete Bereich im folgenden einheitlich als Anfangsbereich bezeichnet. 
6 Numerische Serienberechnungen von Kreiszylinderschalen unter lokaler Belastung

$(a)$

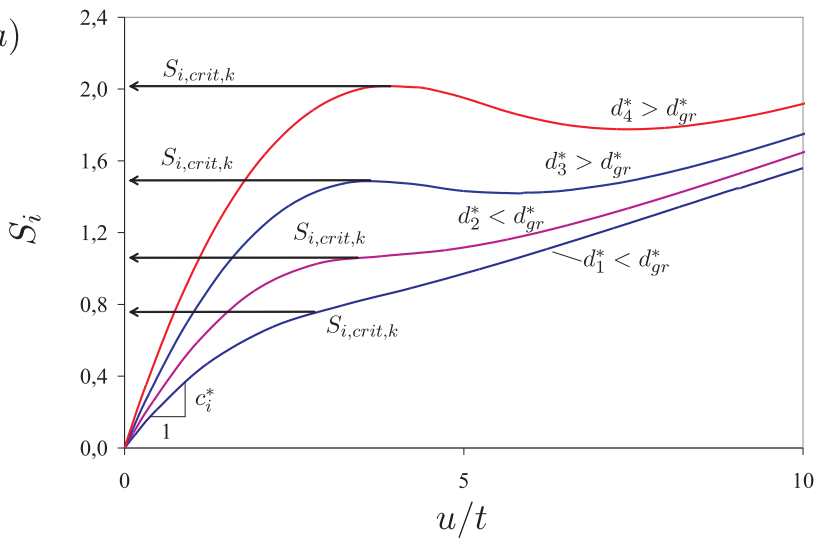

(b)

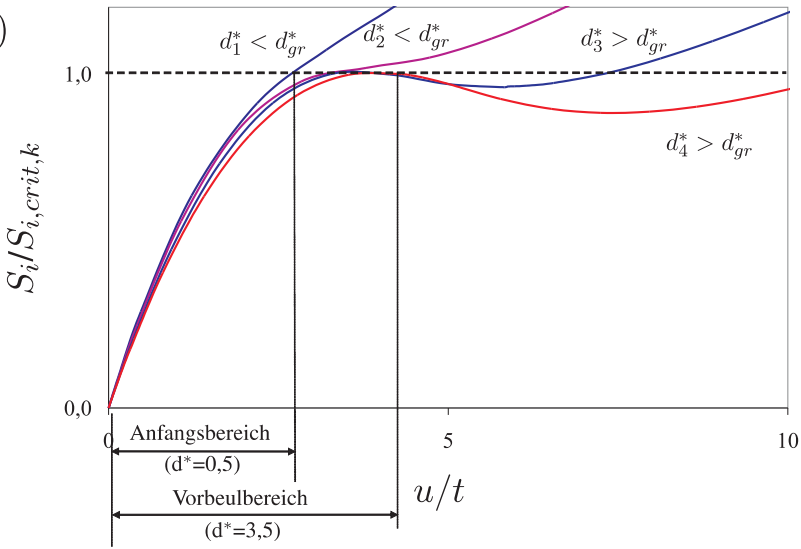

Abbildung 6.1: (a) Last-Verformungskurven; (b) Normierte LastVerformungskurven

\subsection{Kreiszylinderschalen unter radialen Einzella- sten $P_{x}$}

\subsubsection{Allgemeines}

Für die Kreiszylinderschale, die durch radial gerichtete Einzellasten beansprucht wird, werden numerische Berechnungen gemäß den in Abschnitt 6.2 aufgeführten Berechnungskonzepten durchgeführt. Für die numerischen Berechnungen wird 
das in Abschnitt 3.4 beschriebene FE-Modell verwendet. Die in Abschnitt 5.10 begründeten Vereinfachungen wurden dabei berücksichtigt. Die Ergebnisse der geometrisch linearen Berechnungen mit elastischem Werkstoffverhalten $\left(P_{x} \mathrm{LA}\right)$ werden als Spannungsverläufe im Querschnitt der Kreiszylinderschale mit der Stutzenachse entlang des Meridians und des Umfangs dargestellt. Dabei werden die Bogenlänge $s_{\varphi}$ und die Längsachse $z$ durch $\sqrt{R t}$ dividiert. Die Spannungen werden in dimensionsloser Form mit

$$
\sigma_{P_{x}}^{*}=\sigma \frac{t^{2}}{P_{x}}=\frac{\sigma}{E} \frac{R}{t} \frac{1}{P_{x}^{*}}
$$

dargestellt.

Die Ergebnisse der geometrisch nichtlinearen Berechnung ohne und mit Berücksichtigung des plastischen Werkstoffverhaltens ( $P_{x}$ GNA und $P_{x}$ GMNA) werden als Last-Verformungskurven dargestellt. Dabei wird die dimensionslose Last

$$
P_{x}^{*}=\frac{P R}{E t^{3}}
$$

über der dimensionslosen Verschiebung

$$
u^{*}=\frac{u}{t}
$$

aufgetragen. Die Steigung der Last-Verformungskurven im linearen Bereich ist die dimensionslose Anfangssteifigkeit

$$
c_{P_{x}}^{*}=\frac{P_{x}^{*}}{u^{*}}=\frac{P_{x}}{u} \frac{R}{E t^{2}}
$$

Die Ergebnisse der geometrisch linearen Berechnungen bei Berücksichtigung plastischen Werkstoffverhaltens werden als Last-Verformungskurven dargestellt, wobei die dimensionslose plastische Last

$$
\bar{P}_{x}=\frac{P_{x}}{f_{y} t^{2}}
$$

über $u^{*}$ aufgetragen wird.

\subsubsection{Geometrisch lineare Berechnungen mit elastischem Werkstoffgesetz $\left(P_{x} \mathbf{L A}\right)$}

In Abbildung 6.2 sind am Beispiel einer Kreiszylinderschale mit $d^{*}=2,5, L^{*}=30$ und $R / t=400$ die dimensionslosen Umfangs- $\sigma_{\varphi}^{*}$ und Längsspannungen $\sigma_{z}^{*}$ entlang des normierten Umfangs $s_{\varphi} / \sqrt{R t}$ (a), (b) und entlang des normierten Meridians $z / \sqrt{R t}$ (c), (d) aufgetragen. Diese Spannungsverläufe sind typisch für den 
in Abschnitt 6.1 definierten Paramterbereich. Die größten Spannungen sind dabei stets die Umfangsspannungen $\sigma_{\varphi}^{*}$, deren Maxima an der Außenseite der Kreiszylinderschale im Punkt B nach Abbildung 3.6 auftreten. Die Umfangsspannungen sind bedeutend größer als die Längsspannungen, wobei bei den Umfangsspannungen die Biegeanteile gegenüber den Membrananteilen dominieren. Entlang des Umfangs und in Längsrichung klingen die Spannungen schnell ab. Da zudem die Längs- und Umfangsspannungen an der selben Stelle das gleiche Vorzeichen haben, was dazu führt, dass die Vergleichsspannungen an dieser Stelle kleiner als die Umfangsspannungen sind, werden im Folgenden vereinfachend und auf der sicheren Seite liegend nur die Umfangsspannungen und das Spannungsmaximum $\sigma_{\varphi, \max , P_{x}}^{*}$ an der Stelle B betrachtet. Das Maximum ist dabei stets eine Druckspannung. Der Betrag kann aber auf der sicheren Seite liegend auch für die Zugspannungen verwendet werden.

Die Einflüsse der Geometrieparameter $R / t, L^{*}$ und $d^{*}$ auf den Verlauf der Spannungen und das Spannungsmaximum $\sigma_{\varphi, \max , P_{x}}^{*}$ wurden getrennt voneinander untersucht. Dabei wurden jeweils zwei Parameter konstant gehalten, während der dritte Parameter variiert wurde (vgl. Tabelle 6.1).

\begin{tabular}{|c|c|c|}
\hline$R / t$ & $L^{*}$ & $d^{*}$ \\
\hline \hline $400-5000$ & 80 & 2,5 \\
\hline 400 & $10-80$ & 2,5 \\
\hline 400 & 80 & $0,5-3,5$ \\
\hline
\end{tabular}

Tabelle 6.1: Variationsparameter $\left(P_{x} \mathrm{LA}\right)$

Wie sich in den Voruntersuchungen in Abschnitt 5.2 bereits gezeigt hat, beeinflusst das $R / t$-Verhältnis das Last-Verformungsverhalten der Kreiszylinderschale nur wenig. Der ungünstigste Fall wird dabei mit einer Kreiszylinderschale erfasst, deren $R / t$-Verhältnis bei 400 liegt. Dieser Sachverhalt wird durch die bezogenen Umfangsspannung $\sigma_{\varphi}^{*}$, die in Abbildung 6.3 entlang des Umfangs der Kreiszylinderschale mit $L^{*}=80$ und $d^{*}=2,5$ aufgetragen sind, verdeutlicht. Da die Unterschiede sehr klein sind, kann im Folgenden darauf verzichtet werden, unterschiedliche $R / t$-Verhältnisse zu berücksichtigen.

Im nächsten Schritt wurde der Einfluss der bezogenen Länge $L^{*}$ auf den Spannungsverlauf entlang des Umfangs untersucht. Am Beispiel des Spannungsverlaufs einer Kreiszylinderschale mit $R / t=400$ und $d^{*}=2,5$ in Abbildung 6.4 wird ersichtlich, dass sich unterschiedliche bezogene Randabstände $L^{*}$ auf den Spannungsverlauf auswirken. Für $L^{*}=10$ liegt das Spannungsmaximum rund $30 \%$ unterhalb des Spannungsmaximums einer Kreiszylinderschale, deren bezogener Randabstand 80 beträgt. Für $L^{*} \geq 30$ sind die Unterschiede gegenüber dem 


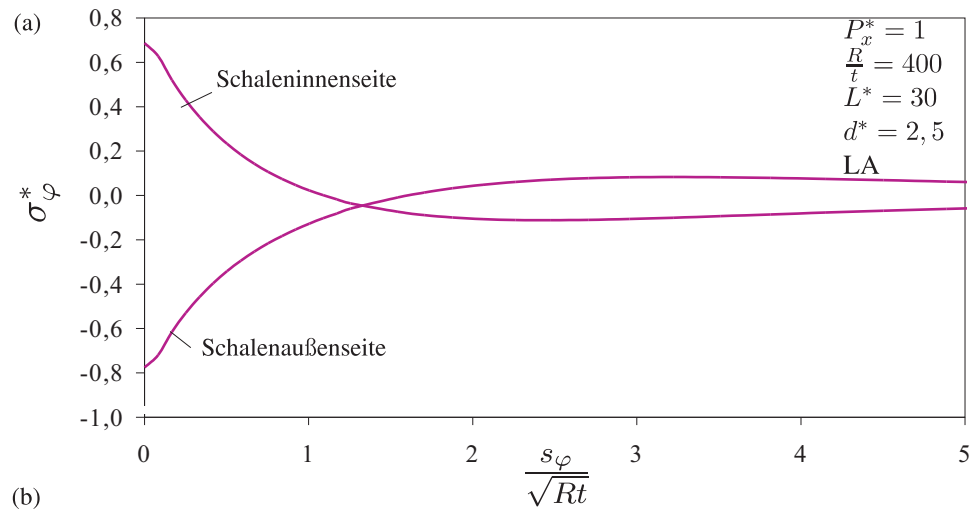

(b)
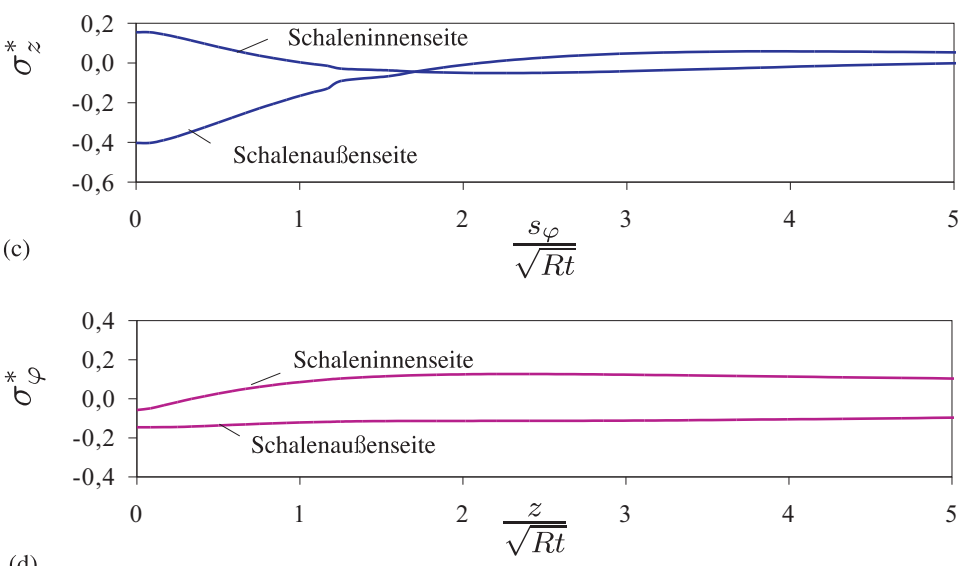

(d)

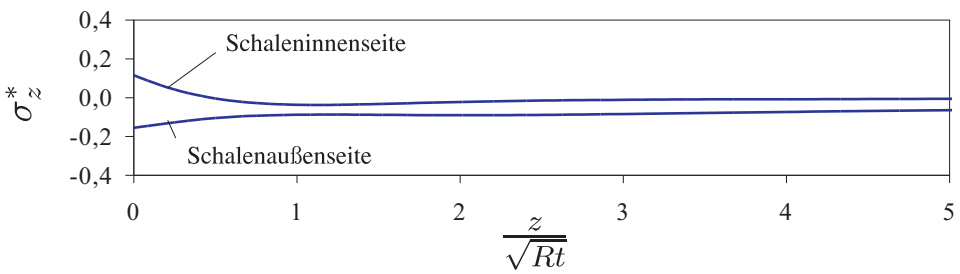

Abbildung 6.2: Verlauf von $\sigma_{\varphi}^{*}$ (a) und $\sigma_{z}^{*}$ (b) entlang des Umfangs und $\sigma_{\varphi}^{*}$ (c) und $\sigma_{z}^{*}$ (d) entlang des Meridians einer Kreiszylinderschale mit $L^{*}=30$, $R / t=400$ und $d^{*}=2,5$ unter einer Last $P_{x}^{*}=1$ 


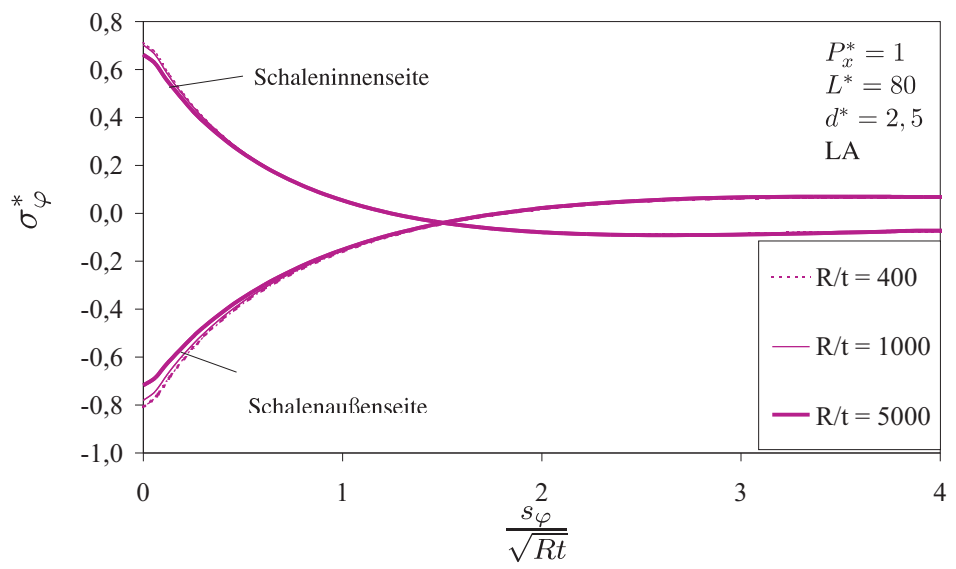

Abbildung 6.3: Verlauf von $\sigma_{\varphi}^{*}$ entlang des Umfangs einer Kreiszylinderschale mit unterschiedlichen $R / t$-Verhältnissen

Spannnungsmaximum $\sigma_{\varphi, \max , P_{x}}^{*}$ einer Kreiszylinderschale mit $L^{*}=80$ so gering, dass sie vernachlässigt werden können.

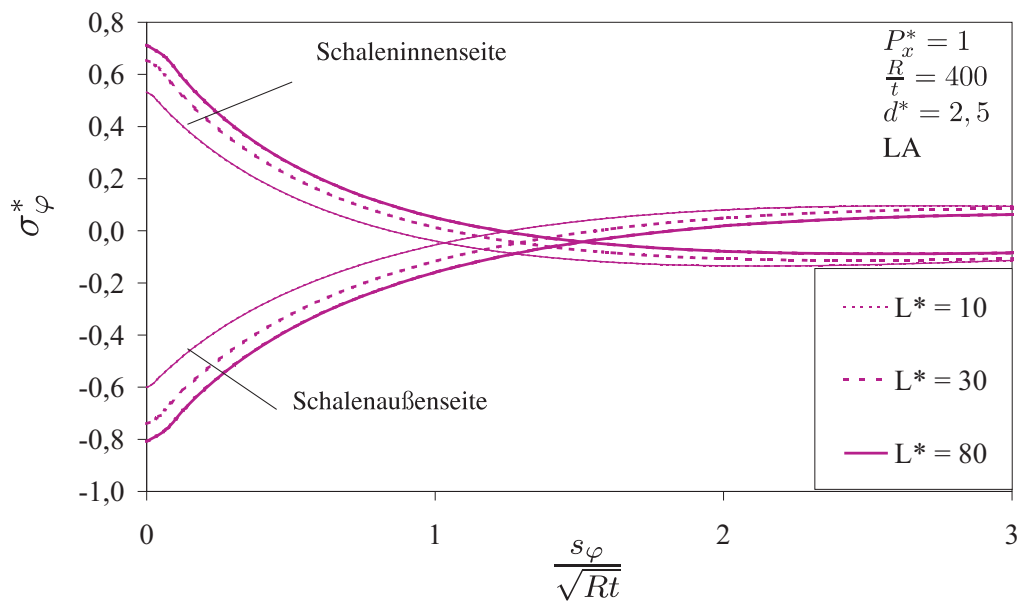

Abbildung 6.4: Verlauf von $\sigma_{\varphi}^{*}$ entlang des Umfangs einer Kreiszylinderschale mit unterschiedlichen bezogenen Randabständen $L^{*}$ 
Für unterschiedliche bezogene Stutzendurchmesser $d^{*}$ sind die Auswirkungen auf den Spannungsverlauf und die Spannungsmaxima am Stutzenanschluss deutlich vorhanden, wie aus Abbildung 6.5 ersichtlich ist. Dort sind für unterschiedliche $d^{*}$ bei konstantem $L^{*}=80$ und $R / t=400$ die bezogenen Umfangsspannungen $\sigma_{\varphi}^{*}$ entlang des Umfangs aufgetragen.

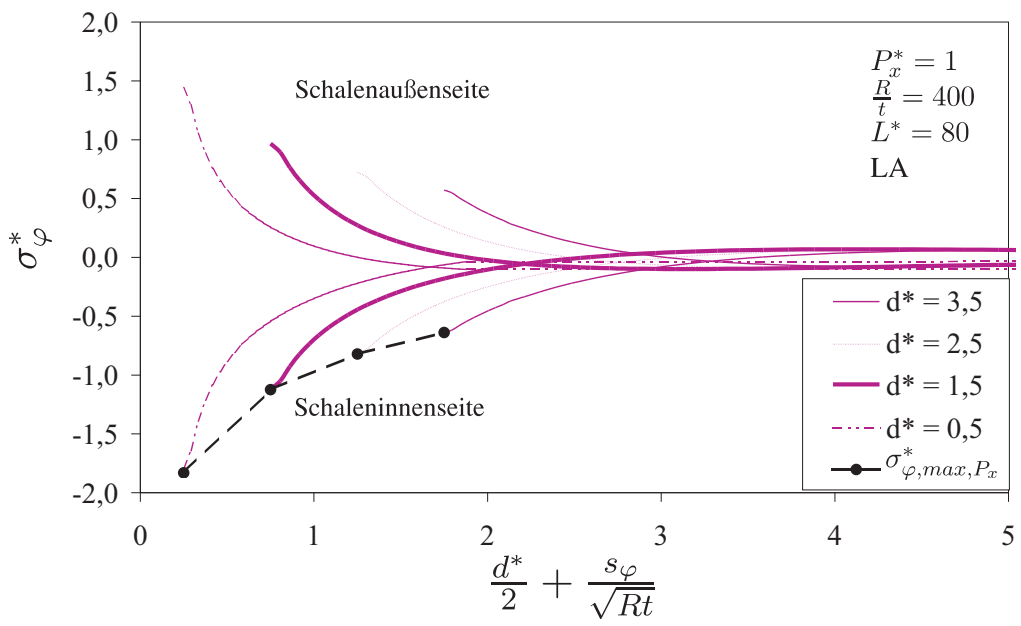

Abbildung 6.5: Verlauf von $\sigma_{\varphi}^{*}$ entlang des Umfangs einer Kreiszylinderschale mit unterschiedlichen bezogenen Stutzendurchmessern $d^{*}$

Wird nun jeweils nur der ungünstigste Fall $\left(L^{*}=80, R / t=400\right)$ betrachtet und werden die Unterschiede, die sich für Kreiszylinderschalen mit $L^{*}<80$ und $R / t>400$ ergeben, vernachlässigt, so kann das Spannungsmaximum $\sigma_{\varphi, \max , P_{x}}^{*}$ in Abhängigkeit von $d^{*}$ mit

$$
\sigma_{\varphi, \max , P_{x}}^{*}=-0,5 d^{*}+2,3 \sqrt{d^{*}}-3,2
$$

abgeschätzt werden.

Die maximalen Umfangsspannungen $\sigma_{\varphi, \max , P_{x}}^{*}$ an der Stelle B, die sich mit Gleichung 6.6 ergeben, sowie die mit Hilfe des WRCB 297 [29] ermittelten Spannungen $\sigma_{\varphi, \max , P_{x}}^{*}$ wurden in Abbildung 6.6 über $d^{*}$ aufgetragen. Für die Spannungsermittlung nach WRCB 297 wurde von einem Stutzen ausgegangen, dessen Wanddicke $t_{r}$ mindestens so dick ist wie das Mantelblech der Kreiszylinderschale. Zusätzlich ist in Abbildung 6.6 die maximale Umfangsspannung $\sigma_{\varphi, \max , P_{x}}^{*}$ einer in [29] dokumentierten FE-Berechnung aufgeführt. Die Spannungsmaxima, die sich nach WRCB 297 ergeben, liegen um 10\% bis 20\% über den Spannungen, die mit Gleichung 6.6 ermittelt wurden. Das Ergebniss der FE-Berechnung 
in [29] liegt dabei zwischen den numerisch ermittelten Spannungen und den mit Hilfe des WRCB 297 ermittelten Spannungen.

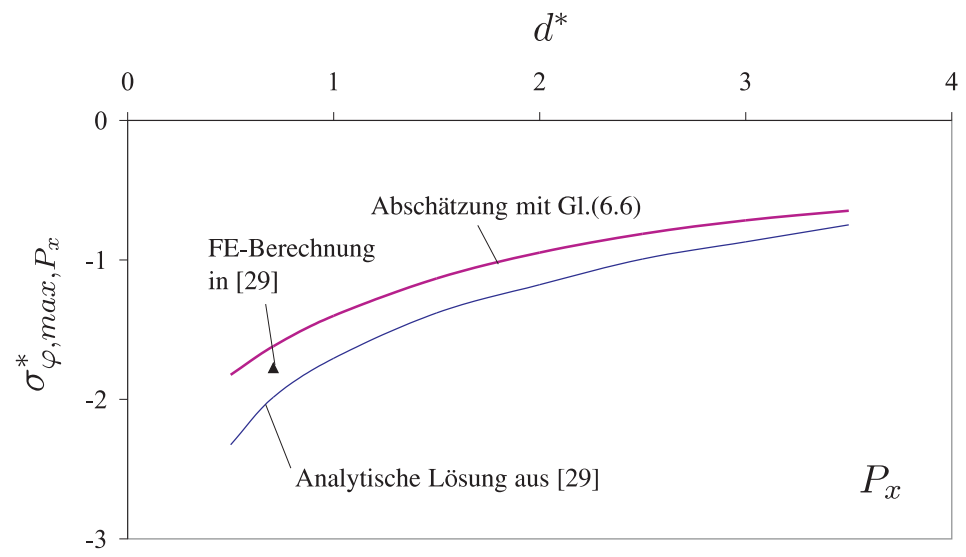

Abbildung 6.6: Vergleich der maximalen Umfangsspannungen nach Gleichung 6.6 und WRCB 297 [29]

Bei der Spannungsermittlung nach WRCB 297 wird nicht zwischen unterschiedlichen bezogene Randabstände $L^{*}$ und $R / t$-Verhältnissen differenziert. Da Gleichung 6.6 bereits vom ungünstigsten Fall $\left(L^{*}=80\right.$ und $\left.R / t>400\right)$ ausgeht, werden mit beiden Verfahren die Spannungen zur sicheren Seite hin abgeschätzt.

\subsubsection{Geometrisch nichtlineare Berechnungen mit elastischem Werkstoffgesetz $\left(P_{x}\right.$ GNA)}

Für die Berechnungen unter Berücksichtigung der geometrischen Nichtlinearität bei elastischem Werkstoffverhalten sind die Variationsparameter in Tabelle 6.2 aufgeführt.

\begin{tabular}{|c|c|c|}
\hline$R / t$ & $L^{*}$ & $d^{*}$ \\
\hline \hline $400-5000$ & 20 & 2,5 \\
\hline 400 & $10-80$ & 2,5 \\
\hline 400 & 20 & $0,5-3,5$ \\
\hline
\end{tabular}

Tabelle 6.2: Variationsparameter $\left(P_{x} \mathrm{GNA}\right)$ 
Da der Einfluss des $R / t$-Verhältnisses auf die Tragfähigkeit sehr gering ist, wie bereits in Abschnitt 5.2 gezeigt wurde, wird dieser Parameter an dieser Stelle nicht untersucht und kann in den folgenden Untersuchungen einen beliebigen Wert zwischen 400 und 5000 annehmen.

Der bezogene Randabstand $L^{*}$ wurde bei konstantem $d^{*}=2,5$ und $R / t=400$ zwischen 10 und 80 variiert. Im nächsten Schritt wurde bei konstantem $L^{*}=20$ und $R / t=400$ der bezogene Stutzendurchmesser für $0,5 \leq d^{*} \leq 3,5$ untersucht. Die Last-Verformungskurven dieser Berechnungen sind in Abbildung 6.7 und Abbildung 6.8 dargestellt. Die Steigung $c_{P_{x}}^{*}$ der Last-Verformungskurven im linearen Bereich nimmt mit zunehmendem $L^{*}$ ab. Je größer $d^{*}$ ist, umso größer ist auch die Steifigkeit der Last-Verformungskurven. Mit steigendem $d^{*}$ stellt sich ein ausgeprägtes lokales Maximum im Kurvenverlauf ein. Dieses Maximum ist die kritische Last $P_{x, c r i t, G N A}^{*}$, die mit steigendem $d^{*}$ zunimmt. Das gleiche Phänomen ist für kleiner werdende $L^{*} \mathrm{zu}$ beobachten.

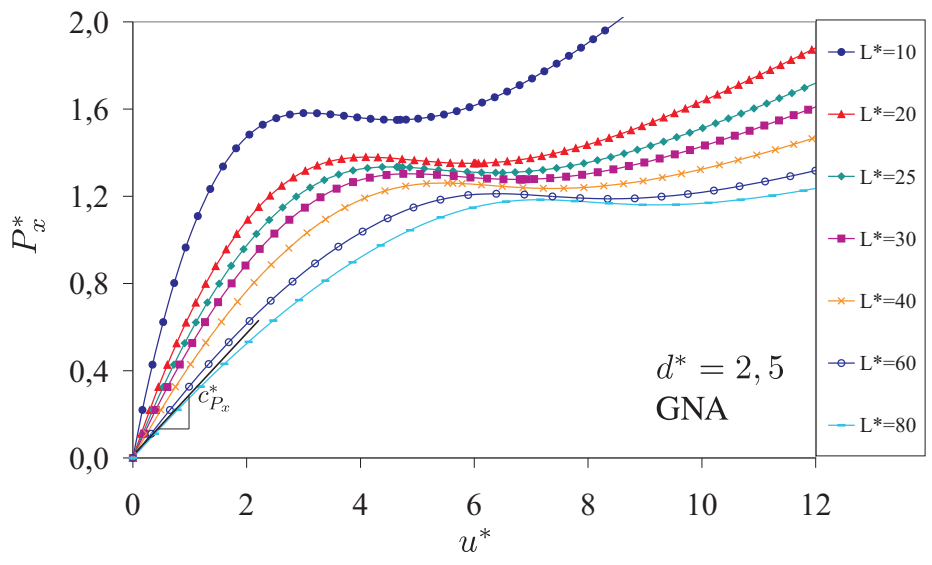

Abbildung 6.7: Last-Verformungskurven von Kreiszylinderschalen unter radialer Einzellast $P_{x}$ mit $d^{*}=2,5$ und $10 \leq L^{*} \leq 80$

Da in Abhängigkeit von $L^{*}$ und $d^{*}$ sich auch die Charakteristik der Last-Verformungskurve ändert, erfolgt die Bestimmung der kritischen Last $P_{x, c r i t, G N A}^{*}$ nach dem in Abschnitt 6.3 beschriebenen Verfahren. Da die Last-Verformungskurven von Kreiszylinderschalen mit $d^{*}<2,5$ kein lokales Maximum besitzen, müssen diese Kurven zur Bestimmung der kritischen Last $P_{x, c r i t, G N A}^{*}$ zunächst normiert werden. In Abbildung 6.9 sind die mit $P_{x, c r i t, G N A}^{*}$ normierten Last-Verformungskurven beispielhaft für $L^{*}=20$ und 
6 Numerische Serienberechnungen von Kreiszylinderschalen unter lokaler

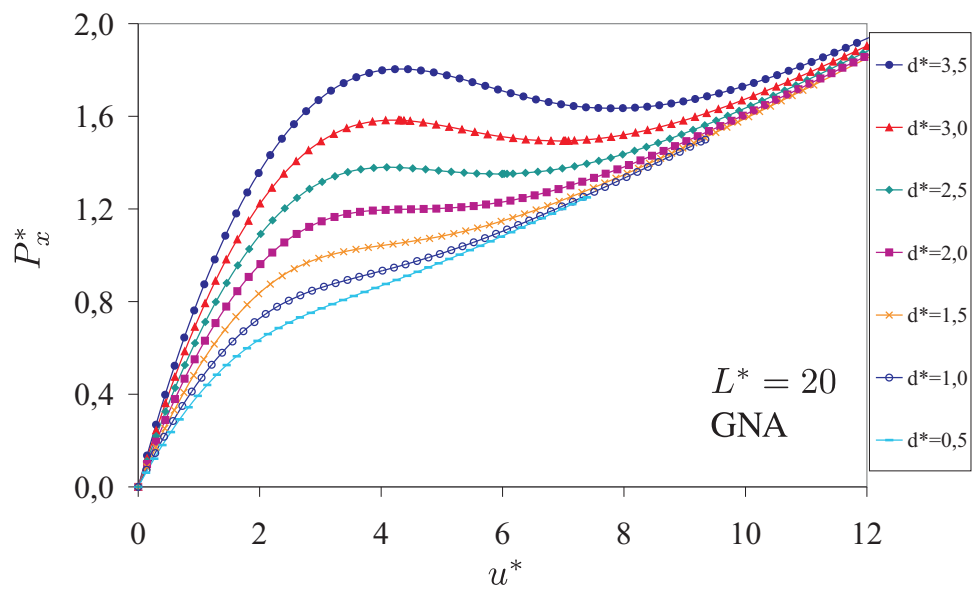

Abbildung 6.8: Last-Verformungskurven von Kreiszylinderschalen unter radialer Einzellast $P_{x}$ mit $L^{*}=20$ und $0,5 \leq d^{*} \leq 3,5$

$0,5 \leq d^{*} \leq 3,5$ und in Abbildung 6.10 für $d^{*}=2,5$ und $10 \leq L^{*} \leq 80$ aufgetragen. Da kein Einfluss aus dem $R / t$-Verhältnis auf das Tragverhalten besteht, kann dieses beliebig innerhalb des untersuchten Bereichs gewählt werden.

Die kritischen Lasten $P_{x, c r i t, G N A}^{*}$ sind in Abbildung 6.11 und die Steigungen $c_{P_{x}}^{*}$ der Last-Verformungskurven im linearen Bereich sind in Abbildung 6.12 für den gesamten untersuchten Parameterbereich über $L^{*}$ aufgetragen. 
6 Numerische Serienberechnungen von Kreiszylinderschalen unter lokaler

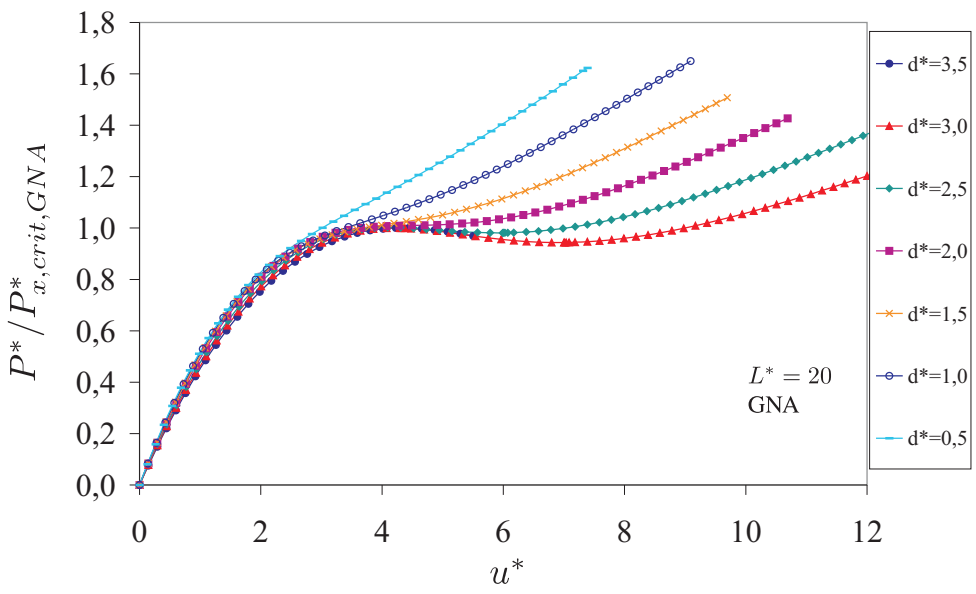

Abbildung 6.9: Normierte Last-Verformungskurven von Kreiszylinderschalen unter radialer Einzellast $P_{x}$ mit $L^{*}=20$ und $0,5 \leq d^{*} \leq 3,5$

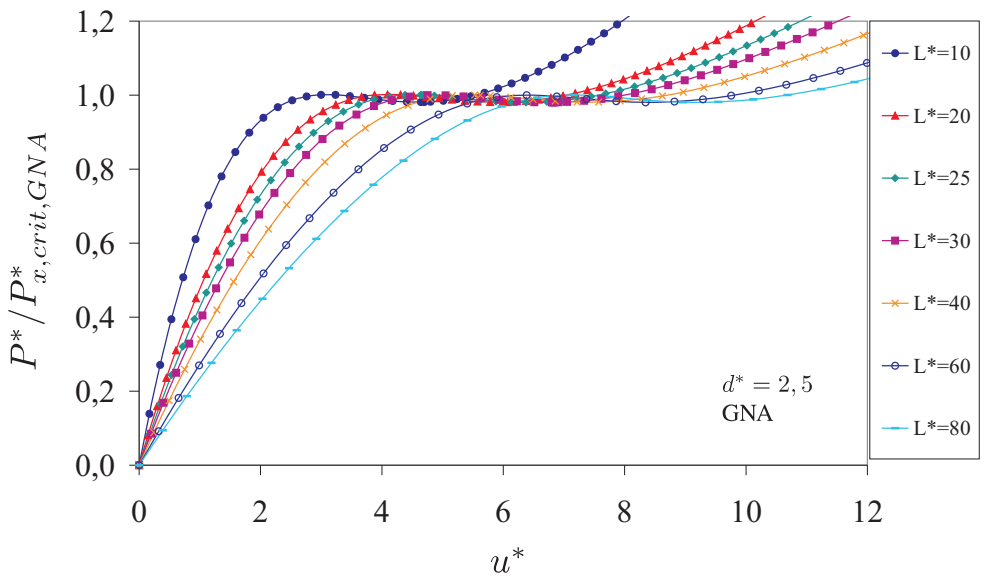

Abbildung 6.10: Normierte Last-Verformungskurven von Kreiszylinderschalen unter radialer Einzellast $P_{x}$ mit $d^{*}=2,5$ und $10 \leq L^{*} \leq 80$ 
6 Numerische Serienberechnungen von Kreiszylinderschalen unter lokaler Belastung

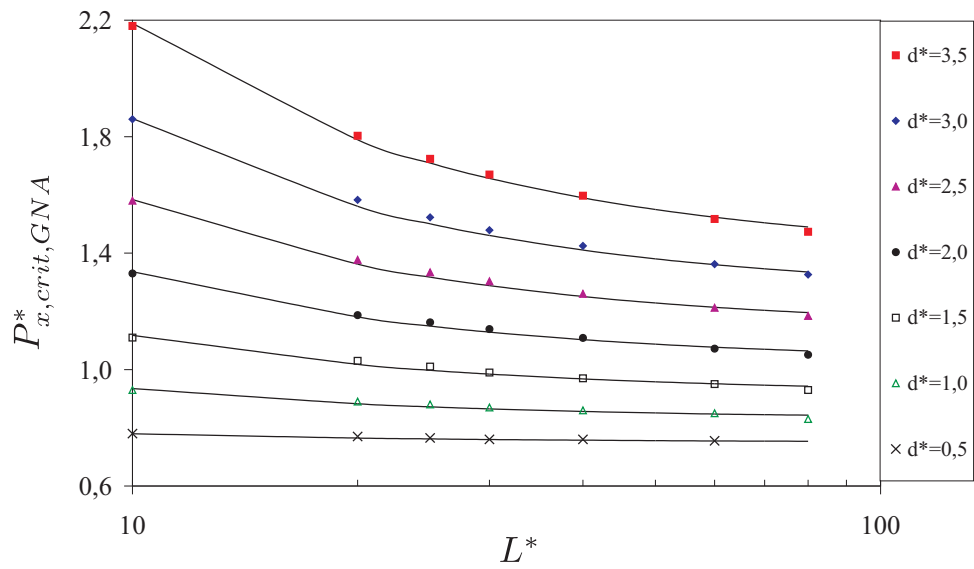

Abbildung 6.11: $P_{x, c r i t, G N A}^{*}$ in Abhängigkeit von $d^{*}$ und $L^{*}$

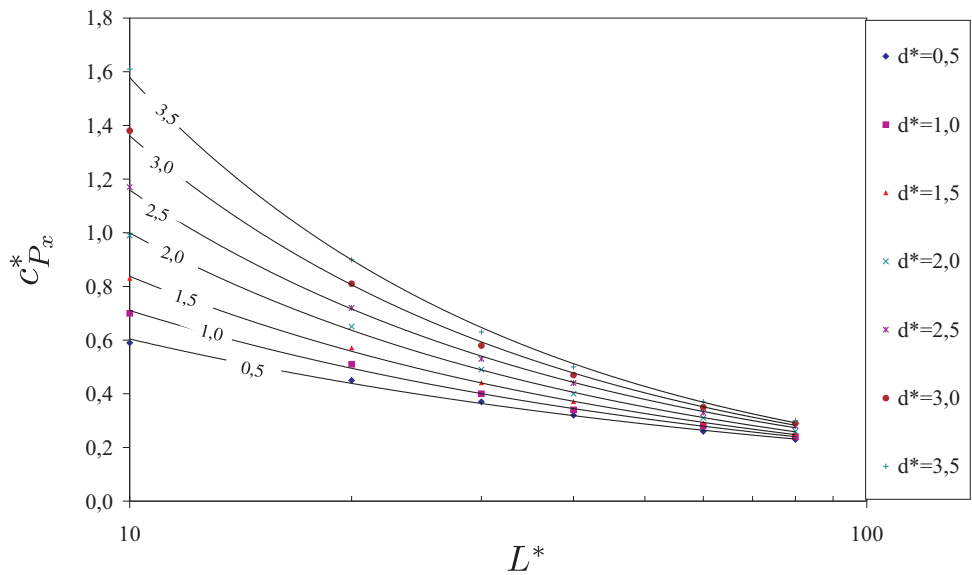

Abbildung 6.12: Anfangssteifigkeit $c_{P_{x}}^{*}$ in Abhängigkeit von $d^{*}$ und $L^{*}$ 


\subsubsection{Geometrisch lineare Berechnungen unter Berücksichti- gung des plastischen Werkstoffgesetzes $\left(P_{x}\right.$ MNA)}

Bei Kreiszylinderschalen mit kleinem $R / t$-Verhältnis und gleichzeitig geringer Streckgrenze $f_{y}$ kann nicht mehr von rein elastischem Werkstoffverhalten im Bereich des Stutzenanschlusses ausgegangen werden. Unter Berücksichtigung des plastischen Werkstoffgesetzes nach Abschnitt 3.5 wurden geometrisch lineare Berechnungen durchgeführt. Dabei wurden die in Tabelle 6.3 aufgeführten Parameter variiert.

\begin{tabular}{|c|c|c|c|}
\hline$R / t$ & $L^{*}$ & $d^{*}$ & $f_{y}$ \\
\hline \hline $400-5000$ & 20 & 2,5 & 240 \\
\hline 400 & $20-80$ & 2,5 & 240 \\
\hline 400 & 20 & $0,5-3,5$ & 240 \\
\hline 1000 & 20 & 2,5 & $240-690$ \\
\hline
\end{tabular}

Tabelle 6.3: Variationsparameter $\left(P_{x} \mathrm{MNA}\right)$

Die Schalenwand plastiziert im Stutzenanschlussbereich. Diese hat zur Folge, dass die Last bei sehr großen Verformungennicht nicht weiter gesteigert werden kann und sich asymptotisch einem Grenzwert nähert. Ein Fließlinienmodell zur Abschätzund der Grenzlast kann in diesem Fall aufgrund der Schalenkrümmung und der Randbedingungen nicht herangezogen werden. Die LastVerformungskurven mit der bezogenen Last $\bar{P}_{x}=P_{x} /\left(f_{y} t^{2}\right)$, die sich aus diesen Berechnungen ergeben, nähern sich asymptotisch einem Grenzwert $\bar{P}_{x, \max , M N A}$. Dabei haben das $R / t$-Verhältnis, der bezogene Randabstand $L^{*}$ und die Streckgrenze $f_{y}$ des Werkstoffes nur einen sehr geringen Einfluss auf die Grenzlast $\bar{P}_{x, \max , M N A}$. Das Beispiel der in Abbildung 6.13 aufgetragenen LastVerformungskurven der Kreiszylinderschalen mit $R / t=400, d^{*}=2,5$ und $20 \leq$ $L^{*} \leq 80$ verdeutlich zudem, dass die Grenzlast $\bar{P}_{x, \text { max }, M N A}$ nur näherungsweise bestimmt werden kann. Aufgrund dieser Unsicherheiten wird auf eine weitere Differenzierung zwischen unterschiedlichen $L^{*}, R / t$ und $f_{y}$ bei der Abschätzung von $\bar{P}_{x, \max , M N A}$ verzichtet.

Durch Variation der bezogenen Stutzendurchmesser $d^{*}$ ändert sich die Grenzlast $\bar{P}_{x, \max , M N A}$, wie Abbildung 6.14 am Beispiel einer Kreiszylinderschale mit $R / t=400, L^{*}=20, f_{y}=240 \mathrm{~N} / \mathrm{mm}^{2}$ und unterschiedlichen $d^{*}$ verdeutlicht. Dabei steigt die Grenzlast $\bar{P}_{x, \max , M N A}$ mit steigendem $d^{*}$ deutlich an. Die Grenzlast $\bar{P}_{x, \max , M N A}$ kann mit

$$
\bar{P}_{x, \max , M N A}=2+4 d^{*}
$$

zur sicheren Seite abgeschätzt werden. 
6 Numerische Serienberechnungen von Kreiszylinderschalen unter lokaler

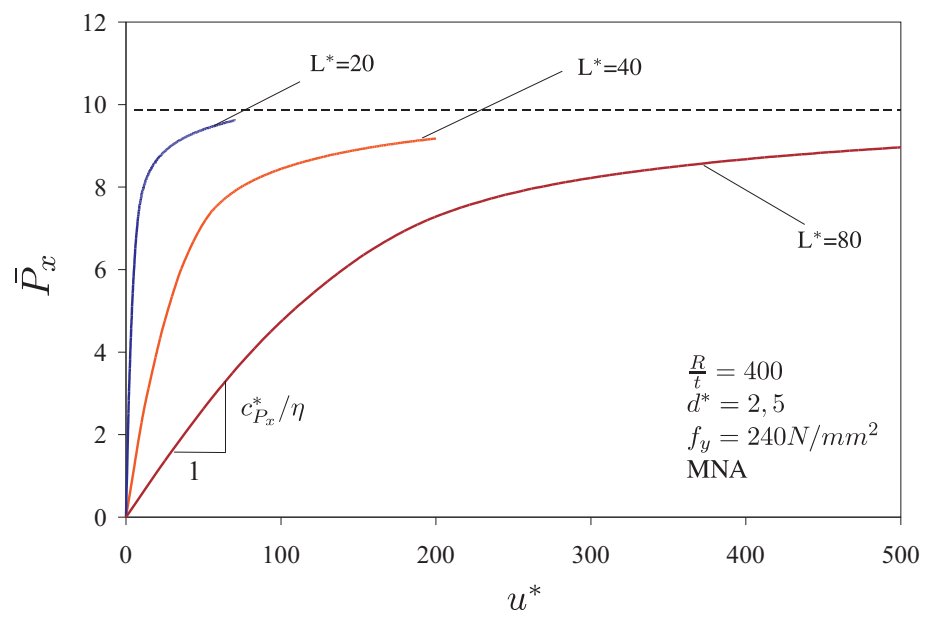

Abbildung 6.13: Last-Verformungskurven von Kreiszylinderschalen unter radialer Einzellast $\bar{P}_{x}$ mit $R / t=400$ und $d^{*}=2,5$ für unterschiedliche $L^{*}$

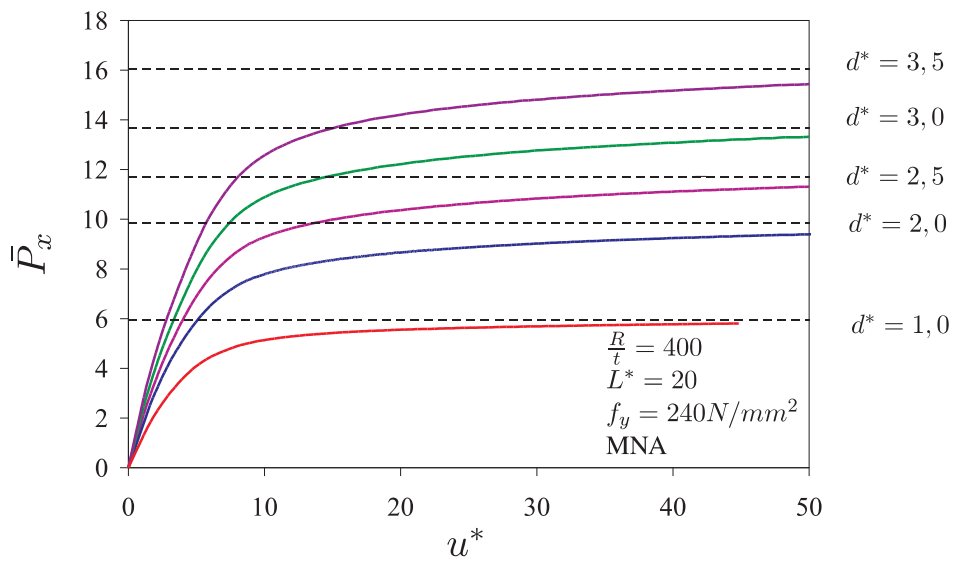

Abbildung 6.14: Last-Verformungskurven von Kreiszylinderschalen unter radialer Einzellast $\bar{P}_{x}$ mit $R / t=400, L^{*}=20, f_{y}=240 \mathrm{~N} / \mathrm{mm}^{2}$ für unterschiedliche bezogene Stutzendurchmesser $d^{*}$ 
Die Anfangssteifigkeit $c_{P_{x}}^{*}$ im linearen Bereich ist unabhängig von der Streckgrenze $f_{y}$. Aufgrund der Normierung der Last $P_{x}$ mit $f_{y} t^{2}$ ergibt sich die Steigung dieser Last-Verformungskurven durch Multiplikation mit $\frac{R}{t} \frac{f_{y}}{E}$. Im Folgenden wird der Zusammenhang zwischen Streckgrenze $f_{y}$, dem E-Modul und dem $R / t$-Verhältnis der Kreiszylinderschale als Plastizitätsfaktor

$$
\eta=\frac{R}{t} \frac{f_{y}}{E}
$$

bezeichnet.

Die mit der Grenzlast $\bar{P}_{x, \max , M N A}$ normierten Kurven, die in Abbildung 6.15 aufgetragen sind, werden umso flacher, je größer $\eta$ oder $L^{*}$ ist. Die erste Abhängigkeit ergibt sich aus

$$
\begin{aligned}
\frac{\bar{P}_{x}}{P_{x}, \max , M N A \cdot u^{*}} & =\frac{P_{x} \cdot t}{f_{y} t^{2} \cdot\left(2+4 d^{*}\right) \cdot u} \\
& =\frac{P_{x} R}{E t^{2} \cdot u} \cdot \frac{E t}{f_{y} R \cdot\left(2+4 d^{*}\right)} \\
& =c_{P_{x}}^{*} \cdot \frac{1}{\eta \cdot\left(2+4 d^{*}\right)}
\end{aligned}
$$

und die zweite aus der Abnahme von $c_{P_{x}}^{*}$ mit $L^{*}$ (vgl. Abbildung 6.12)

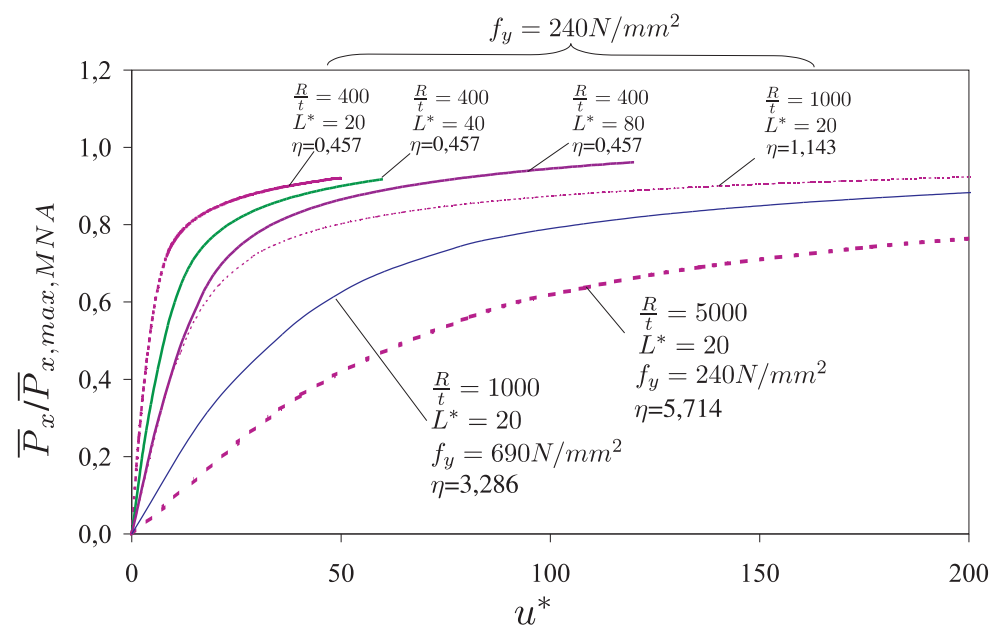

Abbildung 6.16: Normierte Last-Verformungskurven für $d^{*}=2,5$ und unterschiedliche $L^{*}, R / t$ und $f_{y}$ 
6 Numerische Serienberechnungen von Kreiszylinderschalen unter lokaler

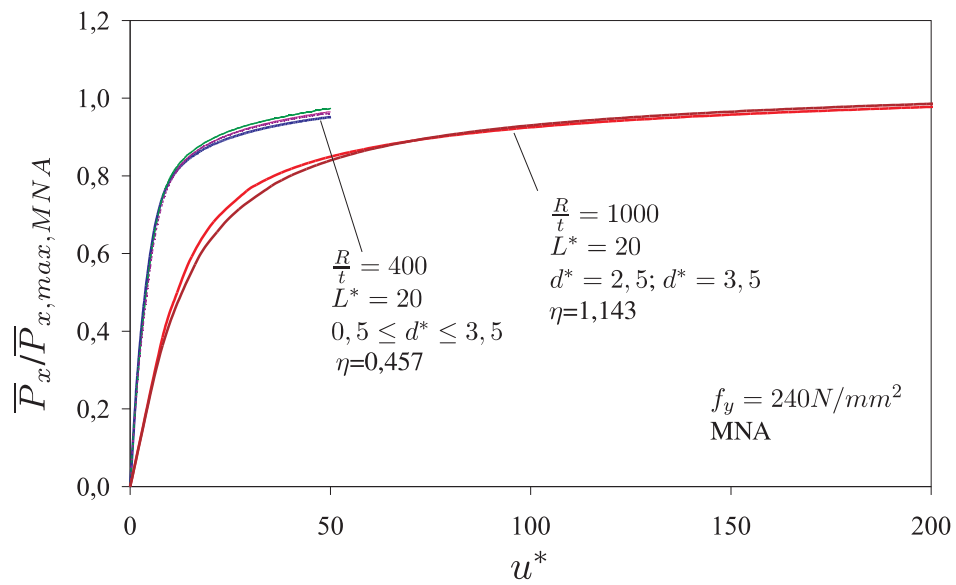

Abbildung 6.15: Normierte Last-Verformungskurven für $d^{*}=2,5, L^{*}=20$, $f_{y}=240 \mathrm{~N} / \mathrm{mm}^{2}$ und unterschiedliche $R / t$

\subsubsection{Geometrisch nichtlineare Berechnungen unter Berück- sichtigung des plastischen Werkstoffgesetzes ( $P_{x}$ GMNA)}

In vielen Fällen beeinflusst die geometrische und materielle Nichtlinearität gleichzeitig das Tragverhalten der Kreiszylinderschale. Dies bedeutet, dass neben dem Einfluss der Geometrieparameter $L^{*}, R / t$ und $d^{*}$ auch der Einfluss der Streckgrenze $f_{y}$ des Stahls eine Rolle spielen kann. Dazu wurden Parameterstudien gemäß Tabelle 6.4 durchgeführt.

\begin{tabular}{|c|c|c|c|}
\hline$R / t$ & $L^{*}$ & $d^{*}$ & $f_{y}$ \\
\hline \hline 400 & $20-80$ & 2,5 & 240 \\
\hline $400-800$ & 20 & 2,5 & 240 \\
\hline 400 & 20 & 2,5 & $240-690$ \\
\hline 400 & 20 & $0,5-3,5$ & 240 \\
\hline
\end{tabular}

Tabelle 6.4: Variationsparameter $\left(P_{x} \mathrm{GMNA}\right)$

Zunächst wurde für eine Kreiszylinderschale mit $R / t=400$ und $d^{*}=2,5$ mit einer Streckgrenze von $f_{y}=240 \mathrm{~N} / \mathrm{mm}^{2}$ der bezogene Randabstand $L^{*}$ variiert. Wie Abbildung 6.17 deutlich zeigt, wird das Tragverhalten der Kreiszylinder- 
schale deutlich vom bezogenen Randabstand $L^{*}$ beeinflusst. Da diese Abhängigkeit auch für die Last-Verformungskurven aus Berechnungen ohne Berücksichtigung des plastischen Werkstoffverhaltens (GNA) besteht, wurden die Streckgrenzen soweit erhöht, dass die maximalen Spannungen weit unterhalb der Streckgrenze $f_{y}$ bleiben. Die Last-Verformungskurven von Kreiszylinderschalen mit $f_{y}=690 \mathrm{~N} / \mathrm{mm}^{2}$ sind daher ebenfalls in Abbildung 6.17 dargestellt. Dabei ist zu erkennen, dass $f_{y}$ das Tragverhalten beeinflusst wenn ein bestimmtes Lastniveau überschritten ist. In diesem Fall plastizieren die Kreiszylinderschale im Stutzenanschlussbereich und die Steifigkeit der Last-Verformungskurven nimmt ab. Dies hat zur Folge, dass die kritische Lasten $P_{x, c r i t, G M N A}^{*}$ im vorliegenden Fall bei Kreiszylinderschalen mit einer Streckgrenze von $f_{y}=240 \mathrm{~N} / \mathrm{mm}^{2}$ um rund $25 \%$ geringer sind, als die kritischen Lasten $P_{x, c r i t, G M N A}^{*}$ der Kreiszylinderschalen mit $f_{y}=690 \mathrm{~N} / \mathrm{mm}^{2}$.

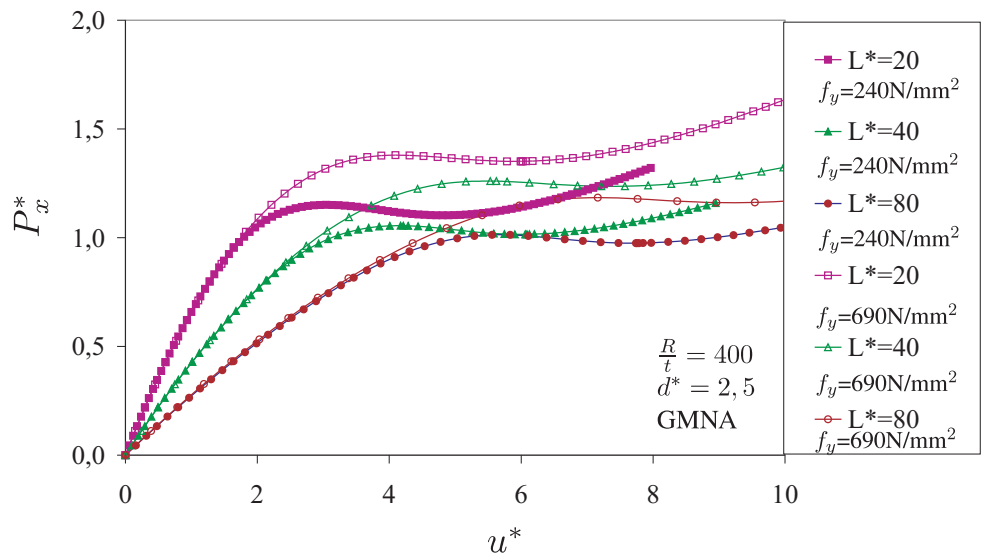

Abbildung 6.17: Last-Verformungskurven von Kreiszylinderschalen mit $R / t=400, d^{*}=2,5$ mit $L^{*}$ zwischen 20 und 80 und Streckgrenzen $f_{y}$ zwischen $240 \mathrm{~N} / \mathrm{mm}^{2}$ und $690 \mathrm{~N} / \mathrm{mm}^{2}$

Im nächsten Schritt wurde eine Kreiszylinderschale mit konstantem $L^{*}=20$ und $d^{*}=2,5$ für unterschiedliche $R / t$ und $f_{y}$ untersucht. Die daraus resultierenden Last-Verformungskurven sind in Abbildung 6.18 dargestellt. Zusätzlich wurde die Tangente im lineraren Anfangsbereich getrichelt eingezeichnet. Im dargestellten Fall zeigen sich die Effekte aus geometrischer Nichtlinearität ab einer Last von etwa $0,6 P_{x}^{*}$. Unterhalb dieser Last sind die Kurven vollkommen linear. Für $P_{x}^{*}>0,6$ weichen die Last-Verformungskurven von diesem linearen Verlauf ab 
und sind für gleiche $L^{*}$ und gleiche $d^{*}$ bis zu einer Last von etwa $1,0 P_{x}^{*}$ vollkommen identisch. Dies bedeutet, dass für Lasten $P_{x}^{*}>0,6$ die geometrische Nichtinearität eine Rolle spielt, die materielle Nichtlinearität dagegen erst ab einem Lastniveau von $1,0 P_{x}^{*}$ einen Einfluss hat. Im geometrisch und materiell linearen Bereich der Last-Verformungskurven haben diese die Anfangssteifigkeit $c_{P_{x}}^{*}$. Der Zusammenhang zwischen $R / t$, dem E-Modul und der Streckgrenze $f_{y}$ wird dabei mit $\eta$ bezeichnet (vgl. Gleichung 6.8).

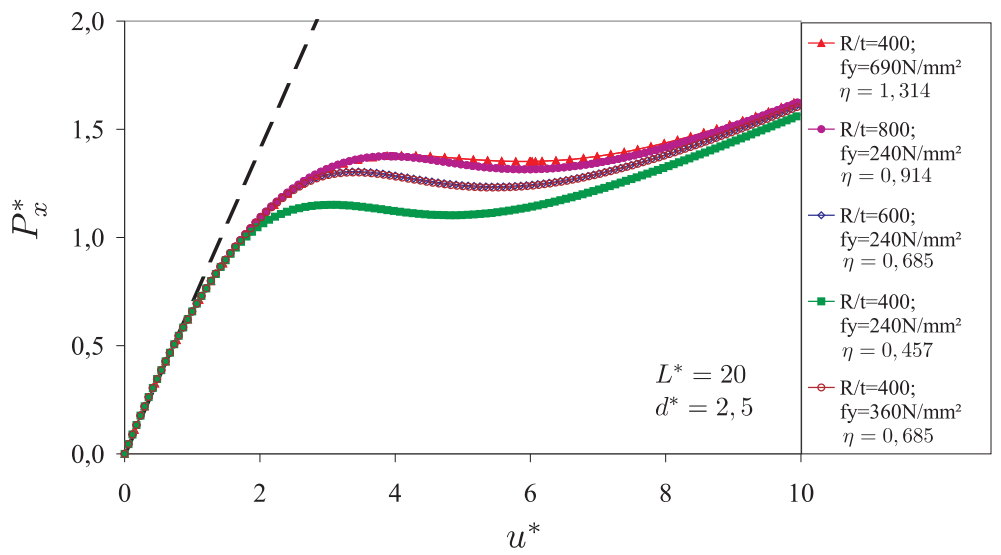

Abbildung 6.18: Last-Verformungskurven von Kreiszylinderschalen mit $L^{*}=20$, $d^{*}=2,5$ für unterschiedliche $R / t$-Verhältnisse und Streckgrenzen $f_{y}$

Die Last-Verformungskurven in Abbildung 6.18 bestätigen den Zusammenhang zwischen kritischer Last und $\eta$. Bei konstantem $d^{*}$ und $L^{*}$ hängt $P_{x, c r i t, G M N A}^{*}$ nur von $\eta$ ab. Schließlich wurde der Einfluss von unterschiedlichen bezogenen Stutzendurchmessern $d^{*}$ auf das Tragverhalten untersucht. Die Last-Verformungskurven von Kreiszylinderschalen mit $L^{*}=20, R / t=400$, $f_{y}=240 \mathrm{~N} / \mathrm{mm}^{2}$ und unterschiedlichen $d^{*}$ sind in Abbildung 6.19 aufgetragen. Es zeigt sich, dass die kritischen Lasten $P_{x, c r i t, G M N A}^{*}$ und die Charakteristik der Last-Verformungskurven von $d^{*}$ beeinflusst werden.

In der normierten Darstellung wurden die Last-Verformungskurven mit der kritischen Last $P_{x, c r i t, G M N A}^{*}$ dividiert und sind in Abbildung 6.20 für unterschiedliche $R / t, L^{*}$ und Streckgrenzen $f_{y}$ aufgetragen. 
6 Numerische Serienberechnungen von Kreiszylinderschalen unter lokaler

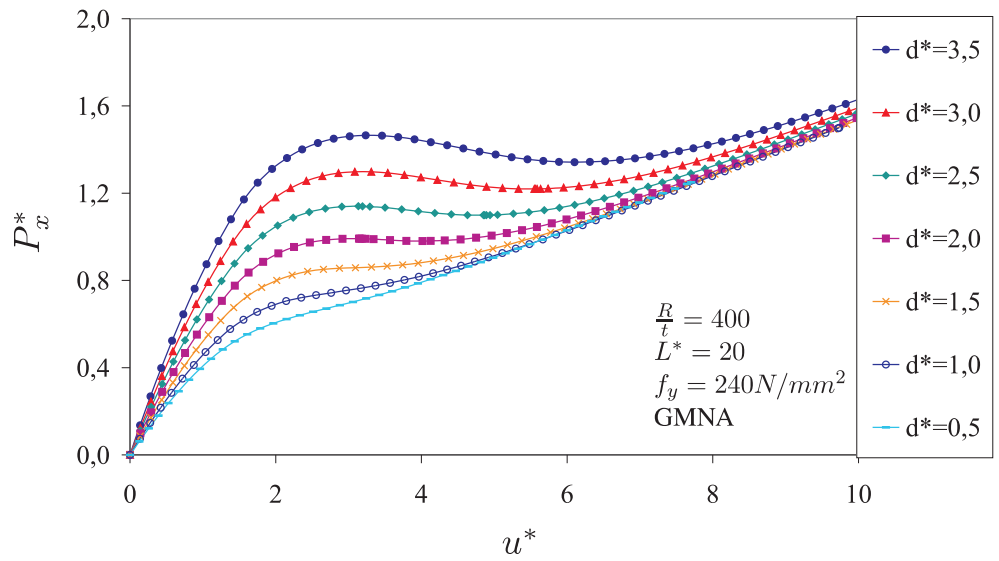

Abbildung 6.19: Last-Verformungskurven von Kreiszylinderschalen mit $L^{*}=20$, $R / t=400, f_{y}=240 \mathrm{~N} / \mathrm{mm}^{2}$ für unterschiedliche bezogenen Stutzendurchmesser $d^{*}$

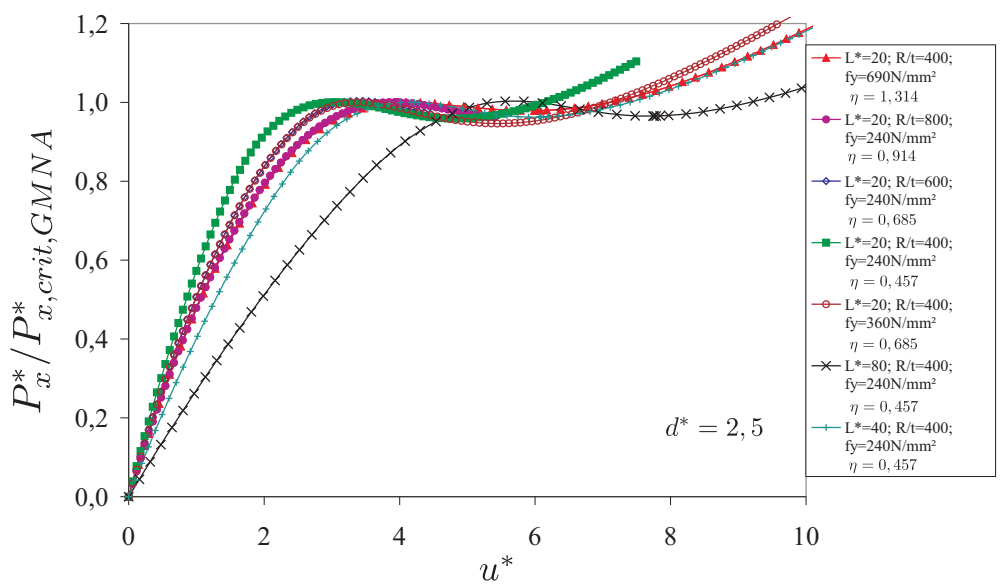

Abbildung 6.20: Normierte Last-Verformungskurven von Kreiszylinderschalen mit $d^{*}=2,5$ für unterschiedliche $R / t, L^{*}$ und $f_{y}$ 


\subsubsection{Interaktion von geometrischer und materieller Nichtli- nearität}

Die normierten Last-Verformungskurven in den Abbildungen 6.9, 6.10, 6.15 6.16 und 6.20 zeigen, dass sich der geometrisch und/oder materiell nichtlineare Bereich deutlich vom linearen Bereich abgegrenzt. Für bezogene Lastgrößen kleiner als 0,4 verlaufen die normierten Last-Verformungskurven linear. Oberhalb dieser Last beginnt der nichtlineare Bereich der Kurven. Dies bedeutet, dass bei bezogenen Lasten $P^{*}$, die größer als $0,4 P_{x, c r i t, G N A}^{*}$ sind, geometrisch nichtlineare Effekte und bei bezogenen Lasten $\bar{P}_{x}$, die größer sind als $0,4 \bar{P}_{x, \max , M N A}$ materiell nichtlineare Effekte eine Rolle spielen.

Die Interaktion der geometrischen und materiellen Nichtlinearität im $\mathrm{Zu}$ sammenhang mit den bezogenen geometrischen Größen $L^{*}$ und $d^{*}$, dem $R / t$-Verhältnis sowie der Streckgrenze $f_{y}$ des Werksoffes verdeutlicht das in Abbildung 6.21 dargestellte Interaktionsdiagramm. Darin ist das Verhältnis der kritischen Lasten $P_{x, c r i t, G M N A}^{*}$ zu $P_{x, c r i t, G N A}^{*}$ über dem Quotienten aus $P_{x, c r i t, G M N A}^{*}$ und $\bar{P}_{x, \max , M N A}$ normiert mit $\eta$ aufgetragen. Bei Kreiszylinderschalen mit großen $R / t$-Verhältnissen und hohen Streckgrenzen ist $P_{x, c r i t, G M N A}^{*} / P_{x, c r i t, G N A}^{*}=1,0$. Das bedeutet, dass die kritischen Lasten im elastischen Bereich liegen. Mit abnehmendem $R / t$-Verhältnis und bei niedrigeren Streckgrenzen wird der Einfluss der materiellen Nichtlinearität auf die kritische Last größer $\left(P_{x, c r i t, G M N A}^{*} / P_{x, c r i t, G N A}^{*}<1,0\right)$, wobei gleichzeitig der Einfluss der geometrischen Nichtlinearität auf die kritischen Lasten abnimmt. Dabei liegen die Verhältniswerte für gleiche bezogene Stutzendurchmesser $d^{*}$ entlang der gestrichelten Geraden in Abbildung 6.21.

Die Verhältniswerte $P_{x, c r i t, G M N A}^{*} / P_{x, \text { crit,GNA }}^{*}$ sind in Abbildung 6.22 über $\eta$ aufgetragen. Dabei stellt die in Abbildung 6.22 an die aufgetragenen Werte angenäherte Kurve eine sichere Abschätzung der Verhältniswerte $P_{x, c r i t, G M N A}^{*} / P_{x, c r i t, G N A}^{*}$ dar. Für $\eta>1,2$ besteht kein Einfluss aus dem plastischen Werkstoffverhalten auf das Tragverhalten der Kreiszylinderschalen.

Die Abminderung der kritischen Lasten $P_{x, c r i t, G M N A}^{*}$ infolge des plastischen Werkstoffverhaltens kann mit

$$
P_{x, c r i t, G M N A}^{*} / P_{x, c r i t, G N A}^{*}=-0,245 \eta^{2}+0,66 \eta+0,56
$$

abgeschätzt werden. 


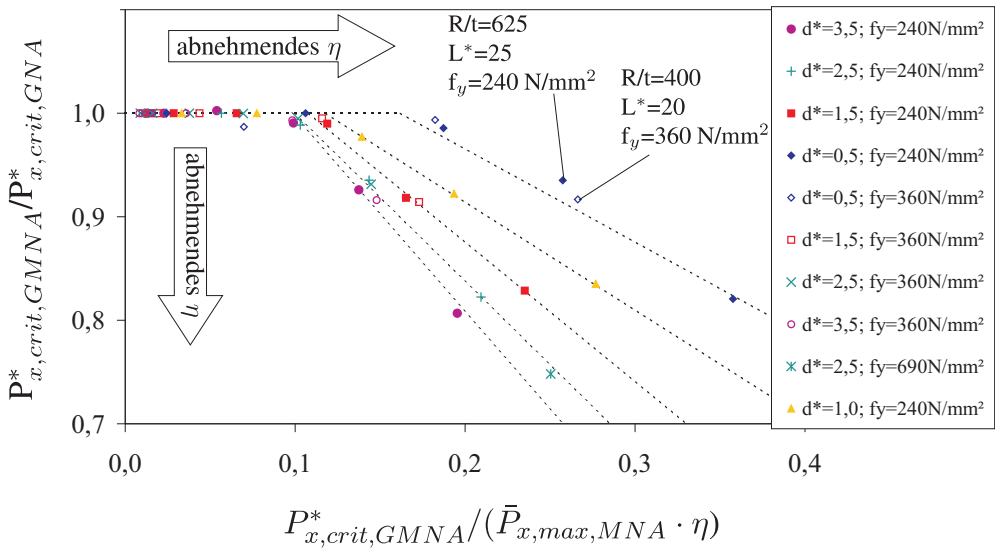

Abbildung 6.21: Interaktion der Grenzlasten $P_{x, c r i t, G M N A}^{*}, \bar{P}_{x, \max , M N A}$ und $P_{x, \text { crit }, G N A}^{*}$

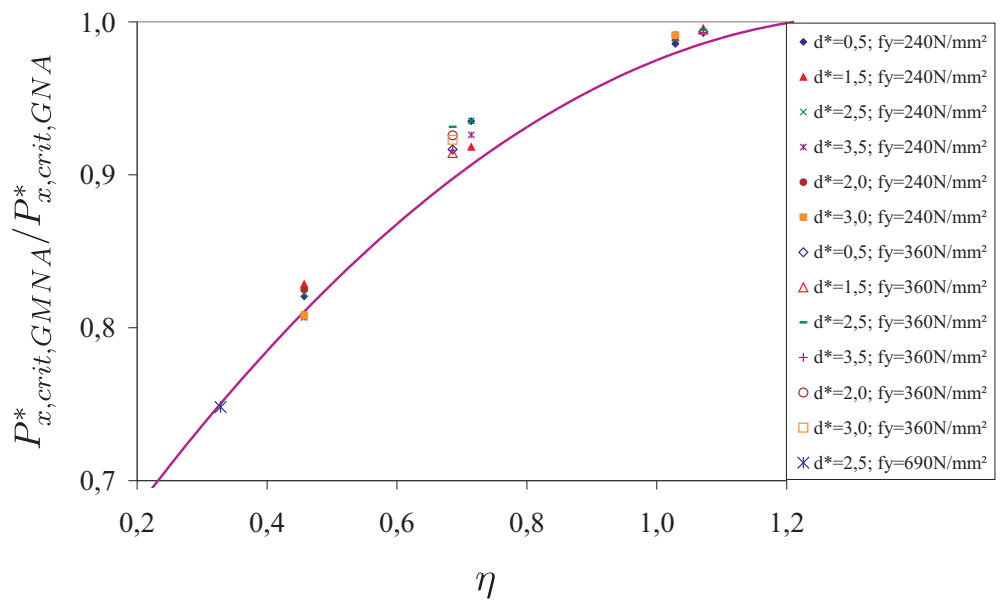

Abbildung 6.22: Einfluss von $R / t$ und $f_{y}$ auf die kritische Last $P_{x, c r i t, G M N A}^{*}$ 


\subsection{Kreiszylinderschalen unter Momentenbela- stung in Umfangsrichtung $M_{\varphi}$}

\subsubsection{Allgemeines}

In diesem Abschnitt werden Kreiszylinderschalen untersucht, an deren Stutzenanschluss ein Moment $M_{\varphi}$ in Umfangsrichtung angreift. Den Untersuchungen liegt das FE-Modell nach Abschnitt 3.4 zugrunde. Die in Abschnitt 5.10 aufgeführten Vereinfachungen wurden bei der Erstellung des FE-Modells berücksichtigt. Das Moment $M_{\varphi}$ in Umfangsrichtung wird in dimensionsloser Form mit

$$
M_{\varphi}^{*}=P_{x}^{*} d^{*}=\frac{M_{\varphi}}{E t^{3}} \sqrt{\frac{R}{t}}
$$

dargestellt.

Die Ergebnisse der geometrisch linearen Berechnungen mit elastischem Werkstoffverhalten $\left(M_{\varphi} \mathrm{LA}\right)$ werden als Spannungsverläufe im Querschnitt der Kreiszylinderschale mit der Stutzenachse entlang des Meridians und des Umfangs dargestellt. Dabei wird die Bogenlänge $s_{\varphi}$ und die Längsachse $z$ durch $\sqrt{R t}$ dividiert. Die Spannungen werden in dimensionsloser Form mit

$$
\sigma_{M_{\varphi}}^{*}=\sigma \frac{d_{r} t^{2}}{M_{\varphi}}=\frac{\sigma}{E} \frac{d^{*}}{M_{\varphi}^{*}} \frac{R}{t}
$$

dargestellt.

Die Ergebnisse der geometrisch nichtlinearen Berechnung ohne und mit Berücksichtigung des plastischen Werkstoffverhaltens $\left(M_{\varphi}\right.$ GNA und $M_{\varphi}$ GMNA) werden als Momenten-Rotationskurven dargestellt. Dabei wird das dimensionslose Moment $M_{\varphi}^{*}$ über der dimensionslosen Verdrehung

$$
\alpha^{*}=\frac{2 u^{*}}{d^{*}}=\alpha \sqrt{\frac{R}{t}}
$$

des Stutzenanschlusses aufgetragen.

Die Steigung der Momenten-Rotationskurven im linearen Bereich wird in dimensionsloser Form als Anfangssteifigkeit

$$
c_{M_{\varphi}}^{*}=\frac{M_{\varphi}^{*}}{\alpha^{*}}=\frac{M_{\varphi}}{\alpha} \frac{1}{E t^{3}}
$$

bezeichnet.

Bei Berücksichtigung nur materieller Nichtlinearität ergibt sich das Moment 
$M_{\varphi}$ in dimensionsloser Form aus

$$
\bar{M}_{\varphi}=\bar{P}_{\varphi} d^{*}=\frac{M_{\varphi}}{f_{y} t^{2} \sqrt{R t}}
$$

\subsubsection{Geometrisch lineare Berechnungen mit elastischem Werkstoffgesetz $\left(M_{\varphi} \mathbf{L A}\right)$}

Der Verlauf der Umfangs- und Längsspannungen einer Kreiszylinderschale mit $L^{*}=30, R / t=1000$ und $d^{*}=2,5$ unter einer Momentenbelastung von $M_{\varphi}^{*}=1$ ist in den Abbildungen 6.23 (a) und (b) entlang des Umfangs und in den Abbildungen 6.23 (c) und (d) entlang des Meridians aufgetragen. Die Spannungen sind dabei als dimensionslose Umfangs- $\sigma_{\varphi}^{*}$ und Längsspannungen $\sigma_{z}^{*}$ dargestellt.

Die größten Spannungen sind dabei die Längsspannungen entlang des Meridians. Das Spannungsmaximum $\sigma_{z, \max , M_{\odot}}$ tritt an der Stelle A an der Außenseite der Kreiszylinderschale auf, wobei die Spannungen zu den Schalenrändern hin rasch abklingen. Die Biegespannungen überwiegen gegenüber den Membranspannungen. Da diese Spannungsverläufe für alle Kreiszylinderschalen im untersuchten Parameterbereich typisch sind, werden für die in Tabelle 6.5 aufgeführte Parameterstudien nur die Längsspannungen $\sigma_{z}^{*}$ entlang des Meridians als maßgebende Spannungen betrachtet.

\begin{tabular}{|c|c|c|}
\hline$R / t$ & $L^{*}$ & $d^{*}$ \\
\hline \hline $400-5000$ & 30 & 2,5 \\
\hline 1000 & $10-80$ & 2,5 \\
\hline 5000 & 80 & $0,5-3,5$ \\
\hline
\end{tabular}

Tabelle 6.5: Variationsparameter $\left(M_{\varphi} \mathrm{LA}\right)$

Durch Variation des $R / t$-Verhältnisses einer Kreiszylinderschale mit $L^{*}=30$ und $d^{*}=2,5$ ändern sich, wie Abbildung 6.24 zeigt. die Spannungsverläufe und Spannungsmaxima geringfügig in Abhängigkeit von $R / t$. Dabei ergeben sich die maximalen Spannungen $\sigma_{z, \max , M_{\varphi}}^{*}$ für das größte untersuchte $R / t$-Verhältnis von 5000. Auf der sicheren Seite liegend werden bei den folgenden Untersuchungen nur Kreiszylinderschalen mit $R / t=5000$ betrachtet und die Differenzen der dimensionslosen Spannungen, die aus unterschiedlichen $R / t$-Verhältnisse resultieren, vernachlässigt.

In Abbildung 6.25 ist der Verlauf der Längsspannungen $\sigma_{z}^{*}$ entlang des Meridians einer Kreiszylinderschale mit $d^{*}=2,5, R / t=1000$ und variablem $L^{*}$ aufgetragen. Dabei zeigt sich, dass die maximale Längsspannung $\sigma_{z, \max , M_{\varphi}}^{*}$ vom bezogenen 
(a)

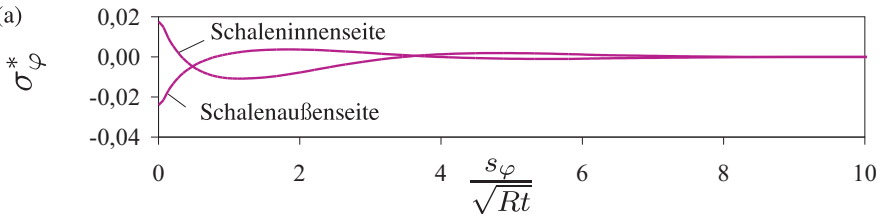

(b) $\quad 0,02 \longrightarrow$ Schaleninnenseite

${ }^{*}{ }^{N}$

Schalenaußenseite

$\begin{array}{rllllll}0 & 2 & 4 & \frac{s \varphi}{\sqrt{R t}} & 6 & 8 & 10\end{array}$

(c)

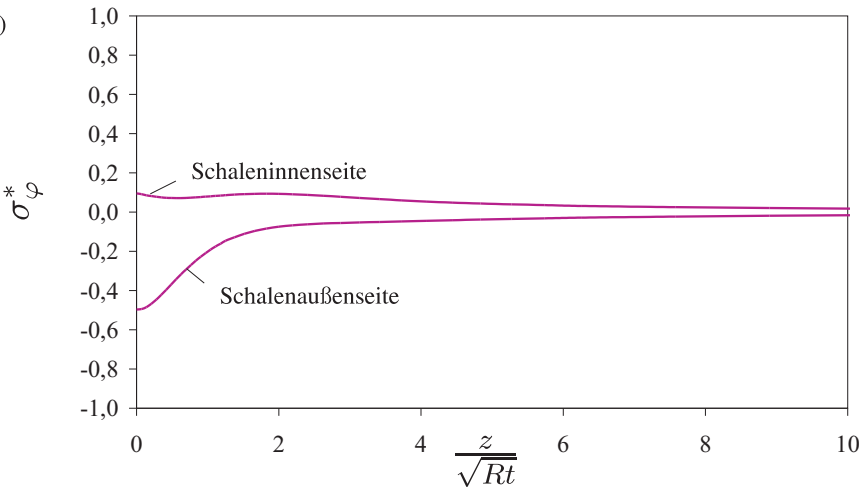

(d)

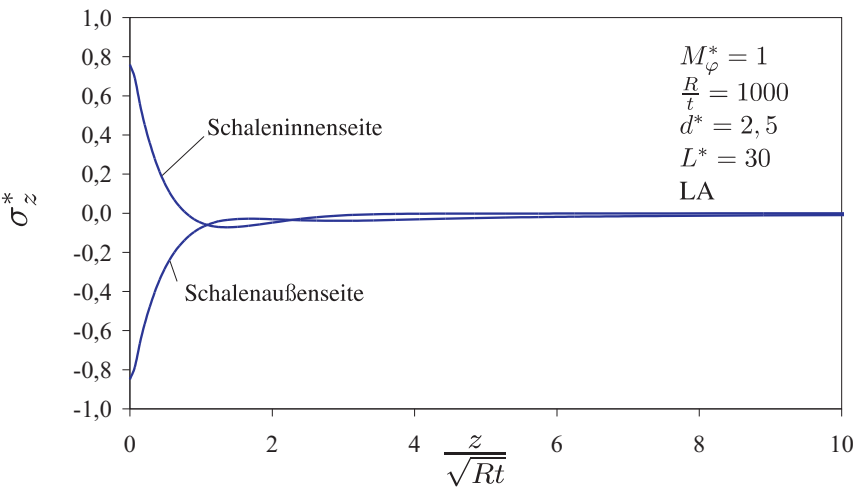

Abbildung 6.23: Verlauf von $\sigma_{\varphi}^{*}$ (a) und $\sigma_{z}^{*}$ (b) entlang des Umfangs und $\sigma_{\varphi}^{*}$ (c) und $\sigma_{z}^{*}$ (d) entlang des Meridians einer Kreiszylinderschale mit $R / t=1000$, $L^{*}=30$ und $d^{*}=2,5$ unter einem Moment von $M_{\varphi}^{*}=1$ 


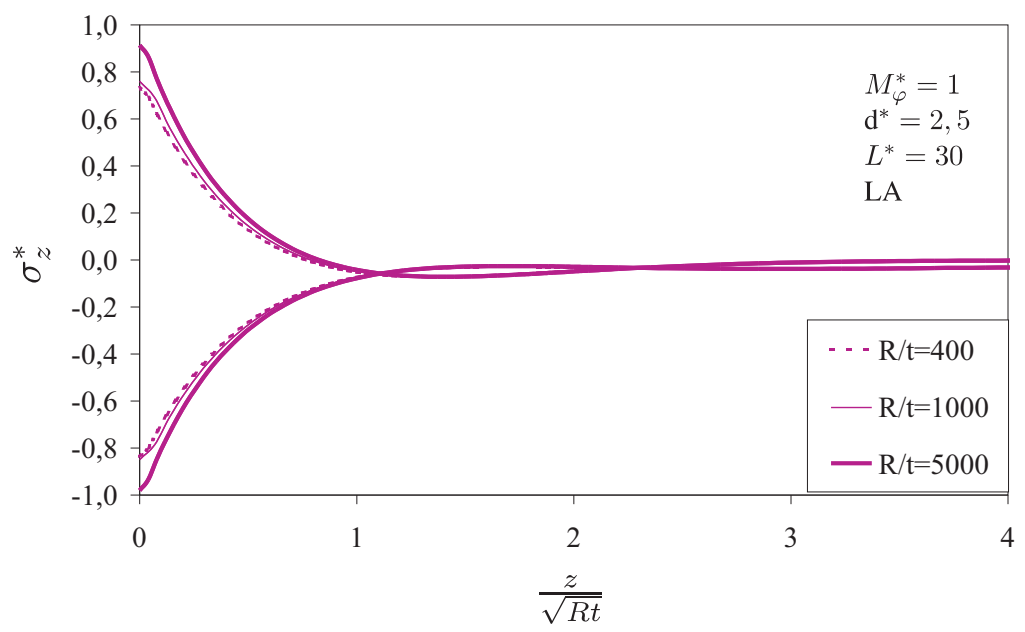

Abbildung 6.24: Verlauf von $\sigma_{z}^{*}$ entlang des Meridians einer Kreizslinderschale mit $d^{*}=2,5, L^{*}=30$ unter einem Moment von $M_{\varphi}^{*}=1$ für unterschiedliche $R / t$

Randabstand $L^{*}$ nicht beeinflusst wird, so dass $L^{*}$ in den folgenden Berechnungen einen beliebigen Wert innerhalb des Parameterbereichs annehmen kann.

Der Verlauf der Längsspannungen $\sigma_{z}^{*}$ entlang des Meridians einer Kreiszylinderschale mit $L^{*}=80, R / t=5000$, der sich für unterschiedliche $d^{*}$ ergibt, ist in Abbildung 6.26 dargestellt. Dabei zeigt sich, dass sich der bezogenen Stutzendurchmesser $d^{*}$ deutlich auf den Spannungsverlauf und die Spannungsmaxima $\sigma_{z, \max , M_{\varphi}}$ auswirkt. 


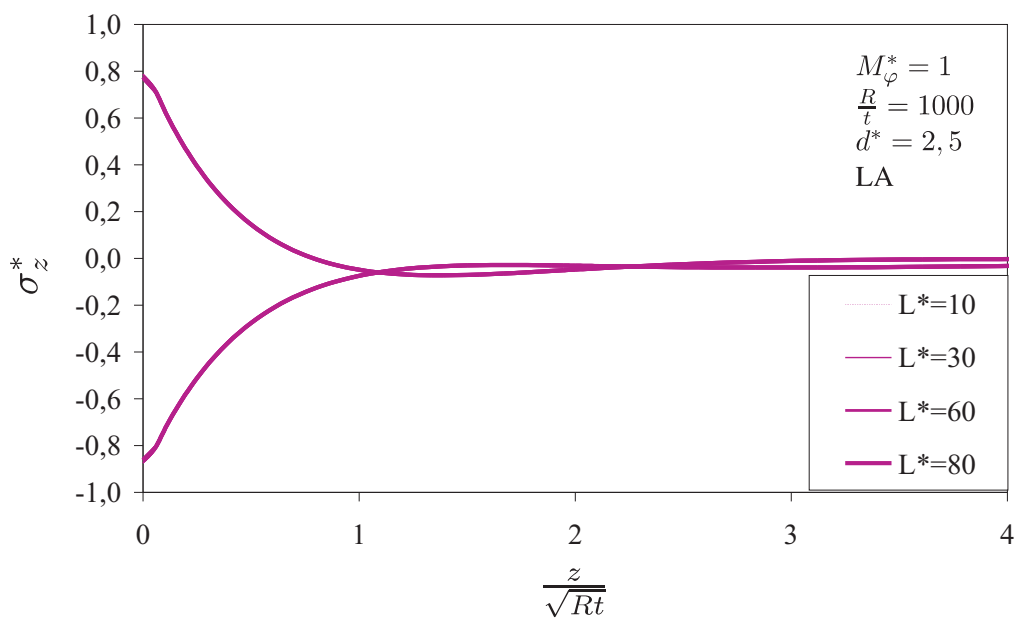

Abbildung 6.25: Verlauf von $\sigma_{z}^{*}$ entlang des Meridians einer Kreiszylinderschale mit $R / t=1000, d^{*}=2,5$ unter einem Moment $M_{\varphi}^{*}=1$ für unterschiedliche $L^{*}$

Da der Biegeanteil gegenüber dem Membrananteil der Längsspannungen im Stutzenanschlussbereich überwiegt, unterscheiden sich die Spannungen an der Innenund Außenseite der Kreiszylinderschale nur unwesentlich. Auf der sicheren Seite liegend werden daher nur die Maximalwerte der Längsspannungen $\sigma_{z, \max , M_{\varphi}}^{*}$ auf der Schalenaußenseite betrachtet. Diese Spannungsmaxima $\sigma_{z, \max , M_{\varphi}}^{*}$ sind in Abbildung 6.26 als Punkte eingetragen und können als Funktion von $d^{*}$ mit

$$
\sigma_{z, \max , M_{\varphi}}^{*}=0,69 \sqrt{d^{*}}-2,1
$$

abgeschätzt werden.

Die maximalen Spannnungen infolge eines am Stutzenanschluss angreifenden Einheitsmomentes $M_{\varphi}^{*}$ wurden nach WRCB 297 [29] an der Außenseite der Kreiszylinderschale ermittelt. Dabei wurde von einem steifen Stutzen ausgegangen, dessen Wanddicke $t_{r}$ mindestens so dick ist wie das Mantelblech der Kreiszylinderschale. In Abbildung 6.27 sind die maximalen Spannungen $\sigma_{z, \max , M_{\varphi}}^{*}$ aus [29] und aus einer in [29] dokumentierten FE-Berechnung, sowie die mit Gleichung 6.15 ermittelten Maximalspannungen über dem bezogenen Stutzendurchmesser $d^{*}$ aufgetragen. 
6 Numerische Serienberechnungen von Kreiszylinderschalen unter lokaler

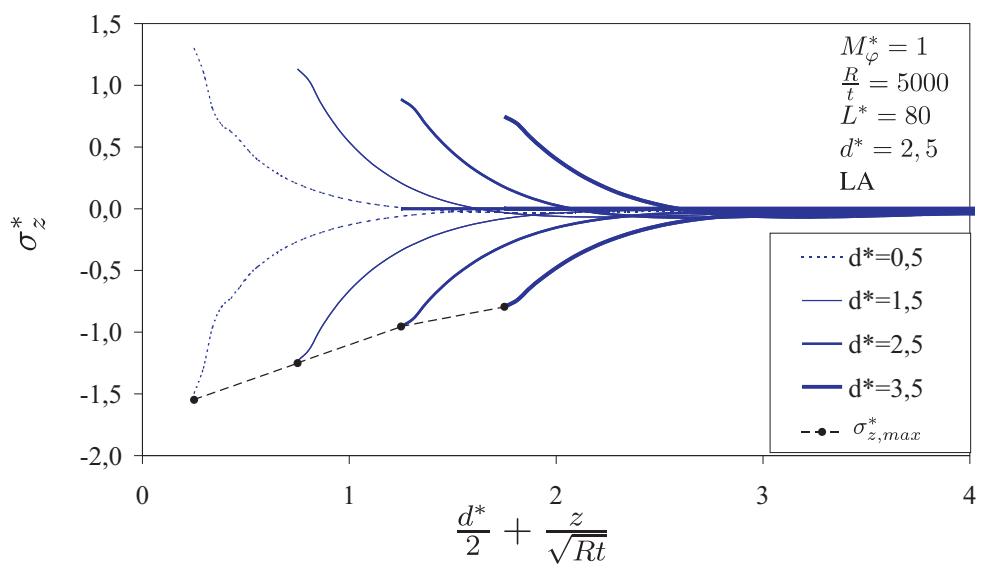

Abbildung 6.26: Verlauf von $\sigma_{z}^{*}$ entlang des Meridians einer Kreiszylinderschale mit $L^{*}=80, R / t=5000$ unter einem Moment $M_{\varphi}^{*}=1$ für unterschiedliche $d^{*}$

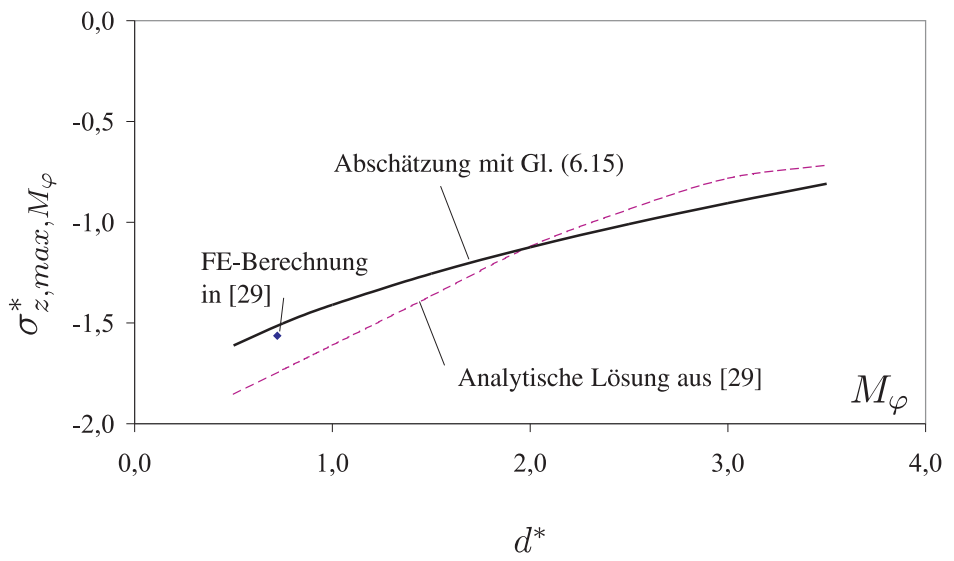

Abbildung 6.27: Maximale Spannungen $\sigma_{z, \max , M_{\varphi}}^{*}$ nach Gleichung 6.15 und WRCB 297 [29] 
Die mittels der FE-Methode ermittelten Spannungen aus [29] stimmen sehr gut mit der Abschätzung der eigenen numerischen Ergebnissen überein. Für $d^{*}>2,0$ besteht auch eine gute Übereinstimmung zwischen numerisch und analytisch ermittelten Ergebnissen. Für $d^{*}<2,0$ liegen die numerischen Ergebnisse dagegen rund $10 \%$ unterhalb der analytischen Ergebnisse aus [29].

\subsubsection{Geometrisch nichtlineare Berechnungen mit elastischem Werkstoffgesetz $\left(M_{\varphi}\right.$ GNA $)$}

Die Untersuchung der Einflüsse aus den Parametern $L^{*}, R / t$ und $d^{*}$ auf das Tragverhalten der Kreiszylinderschale erfolgte anhand des in Tabelle 6.6 aufgeführten Variationsschemas. Zunächst wurden die $R / t$-Verhältnisse der

\begin{tabular}{|c|c|c|}
\hline$R / t$ & $L^{*}$ & $d^{*}$ \\
\hline \hline $400-5000$ & 20 & 2,5 \\
\hline 1000 & $20-80$ & 2,5 \\
\hline 400 & 20 & $0,5-3,5$ \\
\hline
\end{tabular}

Tabelle 6.6: Variationsparameter $\left(M_{\varphi} \mathrm{GNA}\right)$

Kreiszylinderschale bei konstantem $L^{*}$ und $d^{*}$ variiert. Wie Abbildung 6.28 am Beispiel einer Kreiszylinderschale mit $L^{*}=20$ und $d^{*}=2,5$ zeigt, wirken sich unterschiedliche $R / t$-Verhältnisse nicht auf den Kurvenverlauf im Anfangsbereich aus. Die Anfangssteifigkeit $c_{M_{\varphi}}^{*}$ ist wegen der Normierung der Verdrehung $\alpha$ mit $\sqrt{R / t}$ unabhängig vom $R / t$-Verhältnis der Kreiszylinderschale. Bei größeren Verdrehungen des Stutzenanschlussbereiches weichen die Kurven für unterschiedliche $R / t$ vom gemeinsamen Verlauf im Anfangsbereich ab. Da für die Untersuchungen nur der Anfangsbereich mit kleinen und mittleren Verformungen relevant ist, kann daher der Einfluss des $R / t$-Verhältnisses auf das Tragverhalten vernachlässigt werden.

In Abbildung 6.29 sind die Momenten-Rotationskurven einer Kreiszylinderschale mit $R / t=1000, d^{*}=2,5$ und $20 \leq L^{*} \leq 80$ aufgetragen. Dabei zeigt sich, dass der bezogene Randabstand $L^{*}$ keinen Einfluss auf Tragverhalten der Kreiszylinderschalen hat.

Im nächsten Schritt wurde bei konstantem $L^{*}$ und $R / t$ der bezogene Stutzendurchmesser $d^{*}$ variiert. In Abbildung 6.30 sind die Momenten-Rotationskurven für unterschiedliche bezogene Stutzendurchmesser $d^{*}$ einer Kreiszylinderschale 
6 Numerische Serienberechnungen von Kreiszylinderschalen unter lokaler Belastung

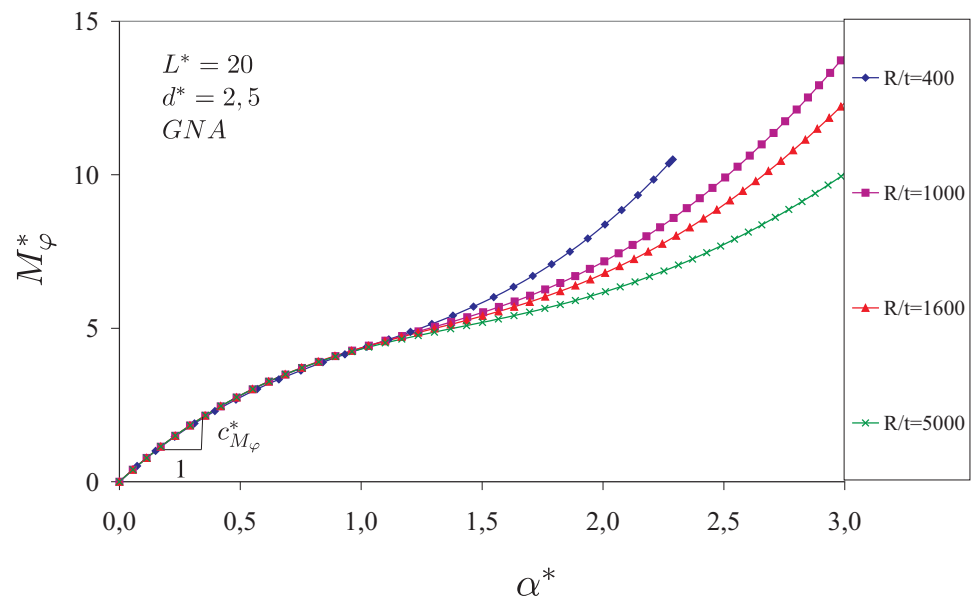

Abbildung 6.28: Momenten-Rotationskurven von Kreiszylinderschalen mit konstantem bezogenen Randabstand $L^{*}=20$ und bezogenem Stutzendurchmesser $d^{*}=2,5$ für $R / t$ zwischen 400 und 5000

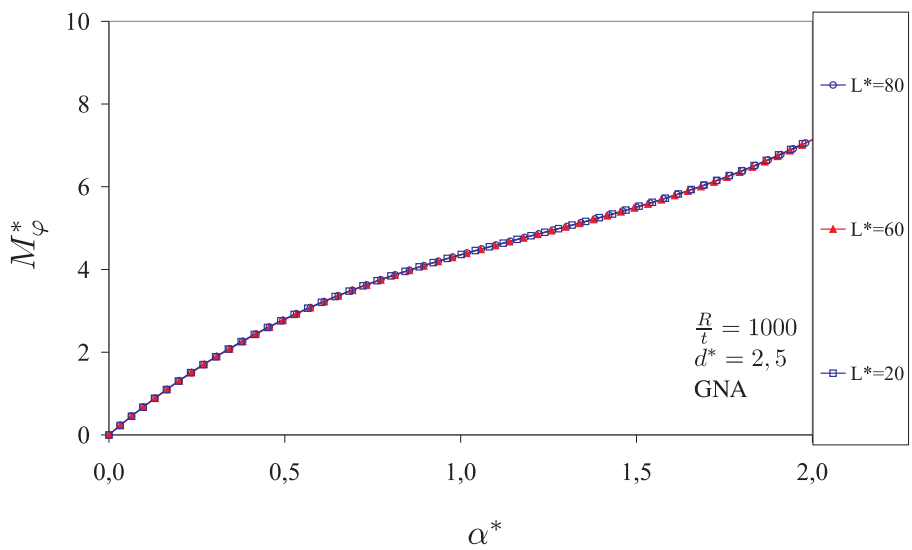

Abbildung 6.29: Momenten-Rotationskurven von Kreiszylinderschalen mit $R / t=1000, d^{*}=2,5$ und unterschiedlichen $L^{*}$ 
mit $L^{*}=20$ und $R / t=400$ dargestellt. Es zeigt sich deutlich, dass das Tragverhalten wesentlich von $d^{*}$ beeinflusst wird. Mit zunehmendem $d^{*}$ steigt auch die Steifigkeit $c_{M_{\varphi}}^{*}$ der Kreiszylinderschale im linearen Bereich. Für $0,5 \leq d^{*} \leq 3,5$ weisen die Kurven kein lokales Maximum im Sinne eines Stabilitätsproblems auf. Durch Erweiterung des Parameterumfangs von $d^{*}$ zeigte sich, dass nur Kreiszylinderschalen, deren bezogener Stutzendurchmesser $d^{*}$ größer als 5,0 ist ein lokales Maxmimum in den Momenten-Rotationskurven haben. Da zur Bestimmung eines kritischen Momentes gemäß dem in Abschnitt 6.3 beschriebenen Verfahren, mindestens eine Momenten-Rotationskurve ein lokales Maximum aufweisen muss, werden für diesen Lastfall zusätzlich zu dem in Abschnitt 6.1 definierten Parameterumfang auch Kreiszylinderschalen mit einem bezogenen Stutzendurchmesser von $d^{*}=5$ untersucht.

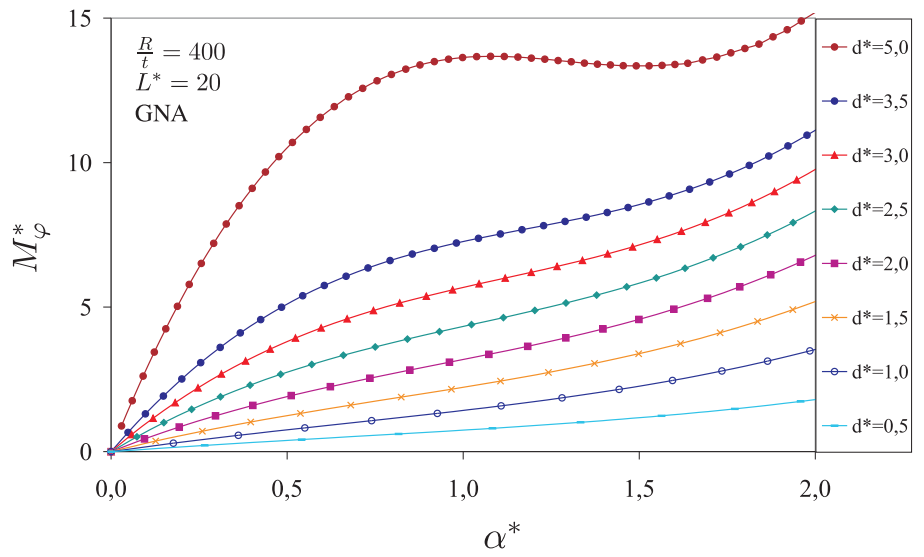

Abbildung 6.30: Momenten-Rotationskurven von Kreiszylinderschalen mit $L^{*}=20, R / t=400$ und bezogenen Stutzendurchmessern zwischen 0,5 und 5,0

Bei der Bestimmung des kritischen Moments $M_{\varphi, c r i t, G N A}^{*}$ können die Einflüsse des $R / t$-Verhältnisses auf das Tragverhalten vernachlässigt werden, da sie nicht im Anfangsbereich vorhanden sind. Dabei erfolgt die Bestimmung des kritischen Moments $M_{\varphi, c r i t, G N A}^{*}$ mit dem in Abschnitt 6.3 beschriebenen Verfahren. In Abhängigkeit von $d^{*}$ ist $M_{\varphi, c r i t, G N A}^{*}$ in Abbildung 6.31 aufgetragen und kann mit

$$
M_{\varphi, \text { crit }, \text { GNA }}^{*}=0,57\left(d^{*}\right)^{2}-0,31 d^{*}+0,47
$$

abgschätzt werden. 


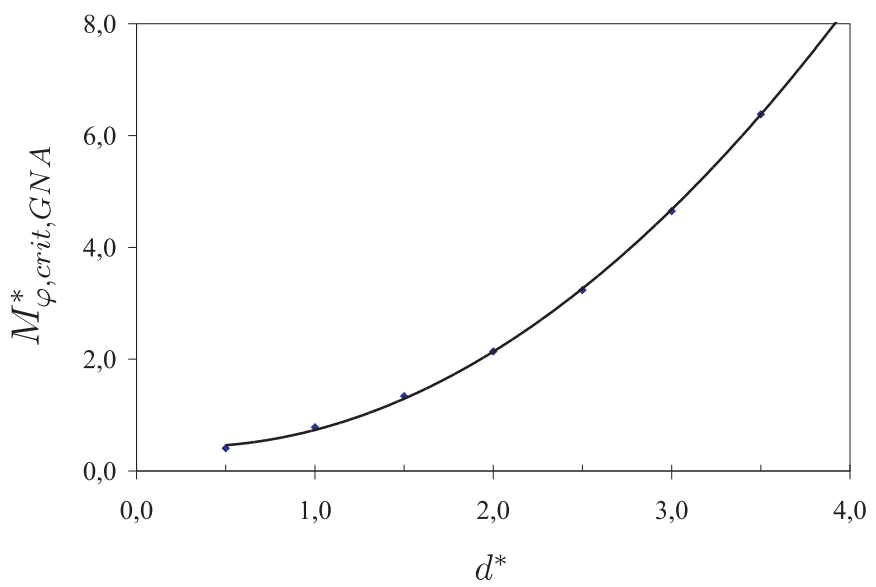

Abbildung 6.31: Kritisches Moment $M_{\varphi, c r i t, G N A}^{*}$ in Abhängigkeit von $d^{*}$

Beim Auftreten des kritischen Moments $M_{\varphi, c r i t, G N A}^{*}$ beträgt die dimensionslose Verdrehung $\alpha^{*}$ etwa 1,0. Dies entspricht einer maximalen radialen Verformung $u^{*}$ der Schalenwand im Stutzenanschlussbereich von etwa $d^{*} / 2$ und damit einer absoluten Verdrehung von etwa $1^{\circ}$ bei $R / t=5000$ bis $3^{\circ}$ bei $R / t=400$.

Die Anfangssteifigkeit $c_{M_{\varphi}}^{*}$ im linearen Bereich der Last-Verformungskurven kann in Abhängigkeit von $d^{*}$ mit

$$
c_{M_{\varphi}}^{*}=1,17\left(d^{*}\right)^{2}-0,64 d^{*}+0,96
$$

abgeschätzt werden.

Die mit dem kritischen Moment $M_{\varphi, c r i t, G N A}^{*}$ normierten MomentenRotationskurven einer Kreiszylinderschale mit $R / t=400$ und $L^{*}=20$ sowie $d^{*}$ zwischen 0,5 und 5,0 sind in Abbildung 6.32 aufgetragen. 


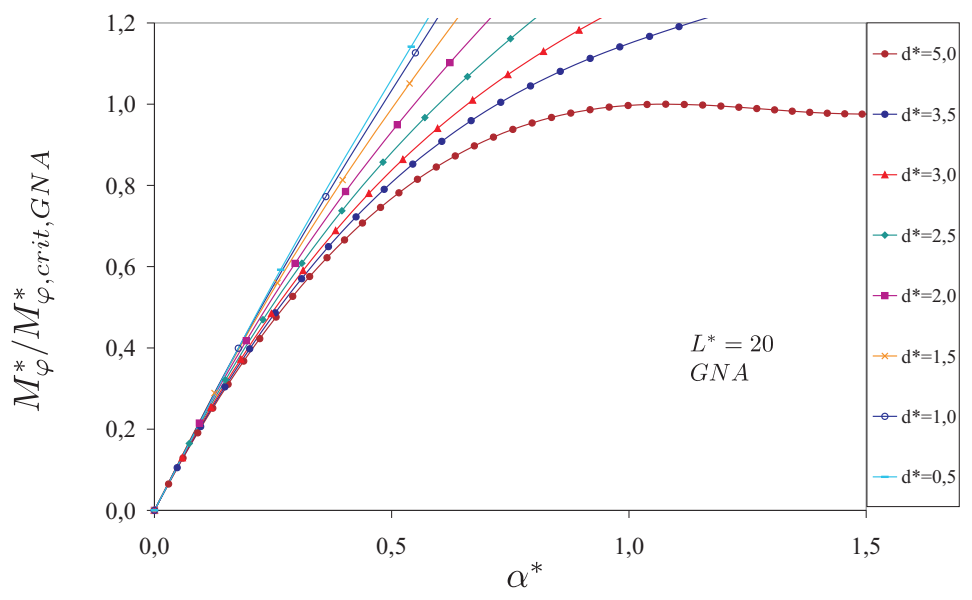

Abbildung 6.32: Normierte Momenten-Rotationskurven von Kreiszylinderschalen mit $R / t=400, L^{*}=20$ und bezogenen Stutzendurchmessern $d^{*}$ zwischen 0,5 und 5,0

\subsubsection{Geometrisch lineare Berechnungen mit plastischem Werkstoffgesetz $\left(M_{\varphi}\right.$ MNA)}

Den Berechnungen liegt das Werkstoffgesetz nach Abschnitt 3.5 zugrunde. Die Berücksichtigung der Streckgrenze $f_{y}$ bei den Berechnungen bedeutet, dass neben den Geometrieparametern auch der Werkstoff einen Einfluss auf das Tragverhalten haben kann. Deshalb wurde in der Parameterstudie neben $L^{*}, R / t$ und $d^{*}$ auch die Streckgrenze $f_{y}$ variiert (vgl. Tabelle 6.7).

\begin{tabular}{|c|c|c|c|}
\hline$R / t$ & $L^{*}$ & $d^{*}$ & $f_{y}$ \\
\hline \hline $400-5000$ & 20 & 2,5 & 240 \\
\hline 400 & 20 & 2,5 & $240-690$ \\
\hline 1150 & $20-80$ & 2,5 & 240 \\
\hline 1000 & 30 & $0,5-3,5$ & 240 \\
\hline
\end{tabular}

Tabelle 6.7: Variationsparameter $\left(M_{\varphi} \mathrm{MNA}\right)$

Zunächst wurde der Einfluss von $L^{*}, R / t$ und $f_{y}$ gemäß der Schritte 1 bis 3 nach Tabelle 6.7 untersucht. Die in Abbildung 6.33 aufgetragenen Momenten- 
Rotationskurven einer Kreiszylinderschale mit $d^{*}=2,5, R / t=1150$ und $f_{y}=240 \mathrm{~N} / \mathrm{mm}^{2}$ mit variablem $L^{*}$ verdeutlichen, dass das Tragvehalten vollkommen unabhängig vom bezogenen Randabstand $L^{*}$ ist.

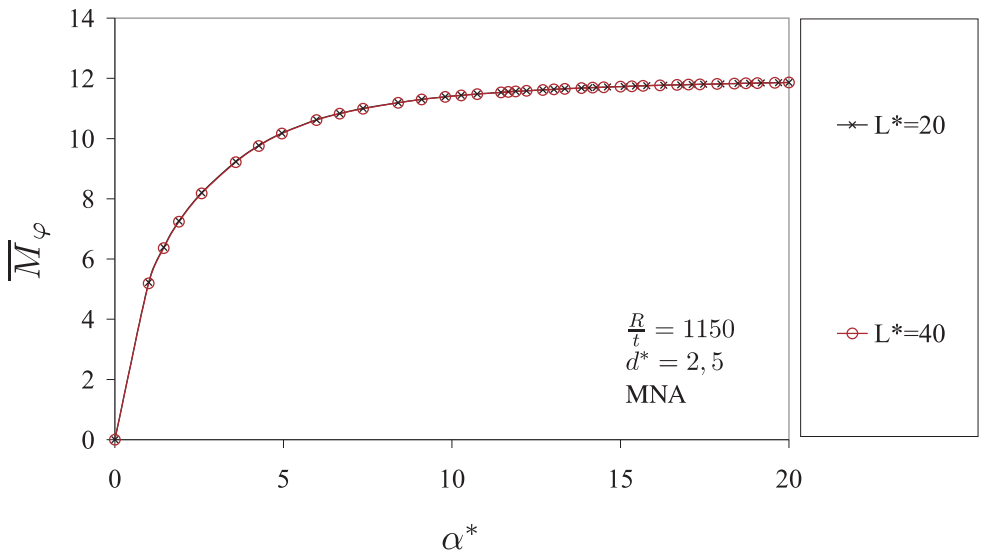

Abbildung 6.33: Momenten-Rotationskurven von Kreiszylinderschalen mit $d^{*}=2,5, R / t=1150, f_{y}=240 \mathrm{~N} / \mathrm{mm}^{2}$ und variablen $L^{*}$

Im nächsten Schritt wurde das $R / t$-Verhältnis und die Streckgrenze $f_{y}$ variiert. Wie die Momenten-Rotationskurven in Abbildung 6.34 einer Kreiszylinderschale mit $d^{*}=2,5$, beliebigem $L^{*}$ und variablen $R / t$ und $f_{y}$ verdeutlichen, ist der Grenzwert $\bar{M}_{\varphi, \max , M N A}$, dem sich die Kurven asymptotisch nähern, vollkommen unabhängig von $L^{*}, R / t$ und $f_{y}$. Wegen der Normierung der Momente mit $f_{y}$ und $t^{2}$ beträgt die Steigung der Kurven im linearen Bereich $c_{M_{\varphi}}^{*} / \eta$.

Im letzten Schritt wurde bei konstantem $R / t=1000, L^{*}=30$ und $f_{y}=240 \mathrm{~N} / \mathrm{mm}^{2}$ der bezogene Stutzendurchmesser $d^{*}$ der Kreiszylinderschale variiert. Die in Abbildung 6.35 aufgetragenen Momenten-Rotationskurven dieser Kreiszylinderschalen zeigen, dass der Grenzwert $\bar{M}_{\varphi, \max , M N A}$ umso größer ist, je größer $d^{*}$ ist.

Das Grenzmoment $\bar{M}_{\varphi, \max , M N A}$ ist unabhängig von $L^{*}$ und $\eta$ und kann in Abhängigkeit von $d^{*}$ in Abbildung 6.36 dargestellt und mit

$$
\bar{M}_{\varphi, \max , M N A}=2,1\left(d^{*}\right)^{2}-1,4 d^{*}+2,6
$$

abgeschätzt werden. 
6 Numerische Serienberechnungen von Kreiszylinderschalen unter lokaler Belastung

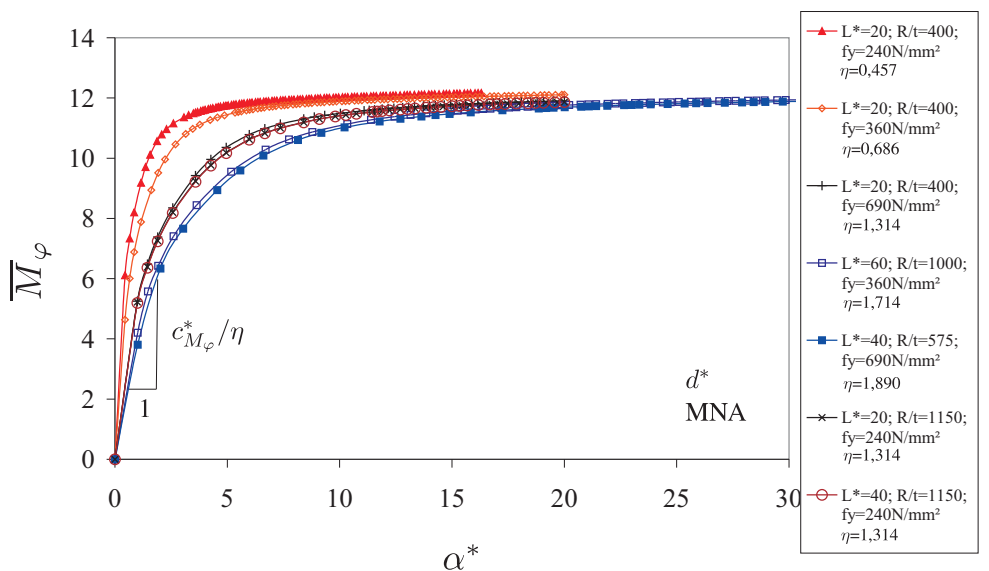

Abbildung 6.34: Momenten-Rotationskurven von Kreiszylinderschalen mit $d^{*}=2,5$ mit variablen $L^{*}, R / t$ und $f_{y}$

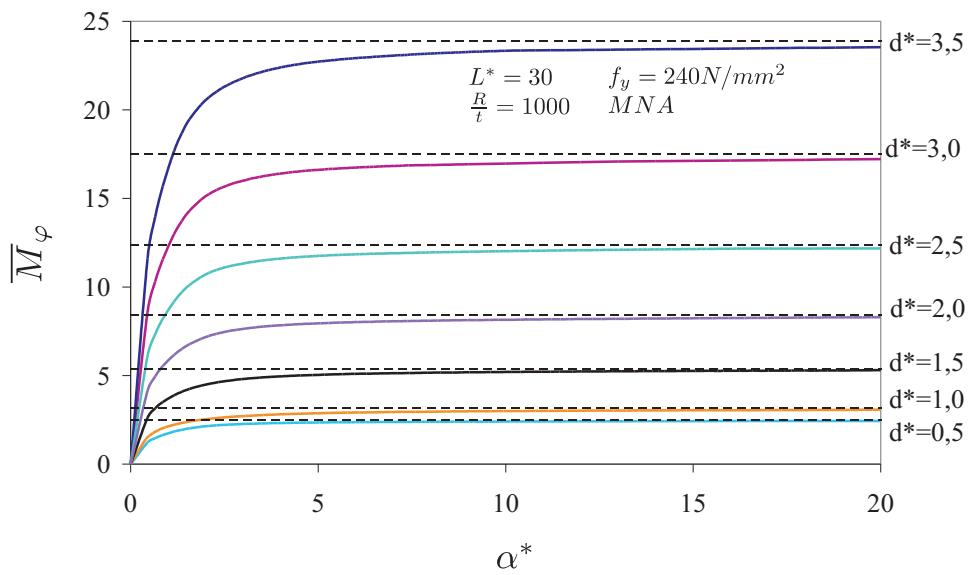

Abbildung 6.35: Momenten-Rotationskurven von Kreiszylinderschalen mit $L^{*}=20, R / t=400, f_{y}=240 \mathrm{~N} / \mathrm{mm}^{2}$ und bezogenen Stutzendurchmessern $d^{*}$ zwischen 0,5 und 3,5 
6 Numerische Serienberechnungen von Kreiszylinderschalen unter lokaler

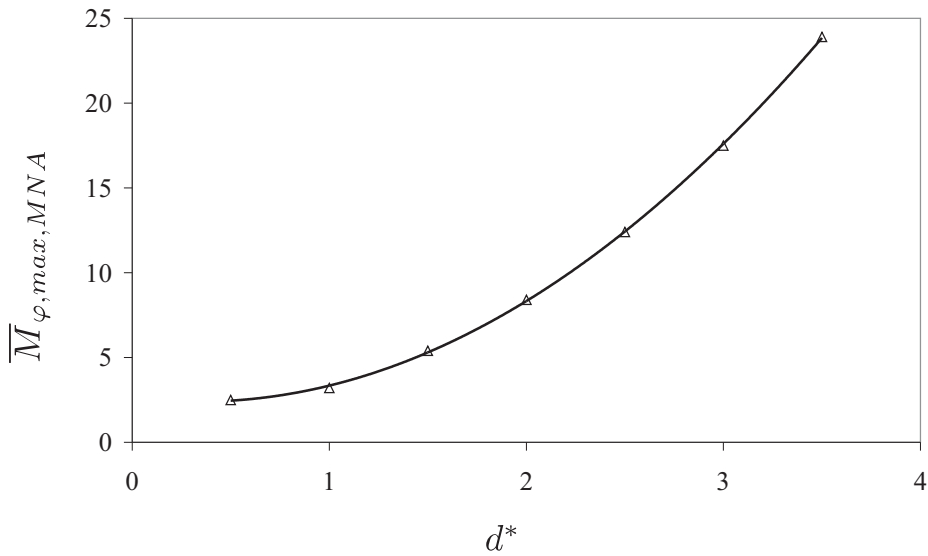

Abbildung 6.36: Maximales Moment $\bar{M}_{\varphi, \max , M N A}$ in Abhängigkeit von $d^{*}$

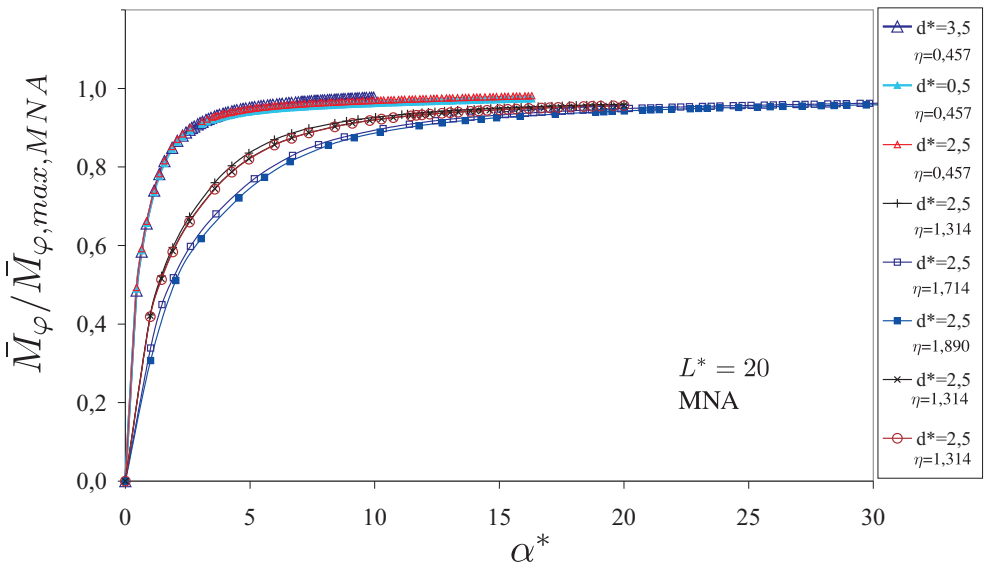

Abbildung 6.37: Normierte Momenten-Rotationskurven von Kreiszylinderschalen mit $L^{*}=20$ und unterschiedlichen $d^{*}$ und $\eta$ 
Die mit dem Grenzmoment $\bar{M}_{\varphi, \max , M N A}$ normierten MomentenRotationskurven sind für gleiche $\eta$ nahezu identisch wie Abbildung 6.37 am Beispiel der Momenten-Rotationskurven von Kreiszylinderschalen mit $L^{*}=20$ und unterschiedlichen $\eta$ sowie unterschiedlichen $d^{*}$ verdeutlicht.

\subsubsection{Geometrisch nichtlineare Berechnung mit plastischem Werkstoffgesetz $\left(M_{\varphi}\right.$ GMNA)}

Da der bezogene Randabstand $L^{*}$ im Lastfall $M_{\varphi}$ bei Berücksichtigung nur geometrischer (Abschnitt 6.5.3) oder nur materieller (Abschnitt 6.5.4) Nichtlinearität keinen Einfluss auf das Tragverhalten hat, ist bei Berücksichtigung von geometrischer und materieller Nichtlinearität ebenfalls kein Einfluss von $L^{*}$ zu erwarten. Folglich wird $L^{*}$ in diesem Abschnitt nicht gesondert untersucht. Der Umfang der Untersuchungen beschränkt sich auf die in Tabelle 6.8 aufgeführten Parameter.

\begin{tabular}{|c|c|c|}
\hline$R / t$ & $d^{*}$ & $f_{y}$ \\
\hline \hline $400-800$ & 3,0 & 240 \\
\hline 400 & 3,0 & $240-360$ \\
\hline 1000 & $0,5-5,0$ & 240 \\
\hline
\end{tabular}

Tabelle 6.8: Variationsparameter $\left(M_{\varphi} \mathrm{GMNA}\right)$

In diesem Fall wurden die Ergebnisse der Schritte 1 bis 3 nach Tabelle 6.8 in einem Diagramm in Abbildung 6.38 aufgeführt. Dabei wird in den Untersuchungsschritten 1 und 2 der Faktor $\eta$ variiert.

Die Kurven haben in Abhängigkeit von $d^{*}$ die gleiche Steigung im linearen Bereich. Die Momenten-Rotationskurven der Kreiszylinderschalen mit $d^{*}=5,0$ und unterschiedlichen $\eta$ weichen ab einer Belastung von $M_{\varphi}^{*}=7,5$ voneinander ab. Bei dieser Last beginnt die Kreiszylinderschale zu plastizieren und wird folglich weicher. Das bedeutet, das kritische Moment $M_{\varphi, c r i t, G M N A}^{*}$ wird vom plastischen Faktor $\eta$ beeinflusst. Bei Kreiszylinderschalen mit kleinen bezogenen Stutzendurchmessern $d^{*}<5$ weichen die Kurven mit kleinem $\eta$ unter großer Verformungszunahme von denen mit einem größeren $\eta$ ab. Es tritt jedoch kein lokales Maximum auf. Das kritische Moment wird in diesen Fällen indirekt über die normierten Kurven bestimmt (vgl. Abschnitt 6.3). Das bedeutet, dass neben $\eta$ auch der bezogene Stutzendurchmesser $d^{*}$ das kritische Moment $M_{\varphi, \text { crit }, \text { GMNA }}^{*}$ beeinflusst. 
6 Numerische Serienberechnungen von Kreiszylinderschalen unter lokaler

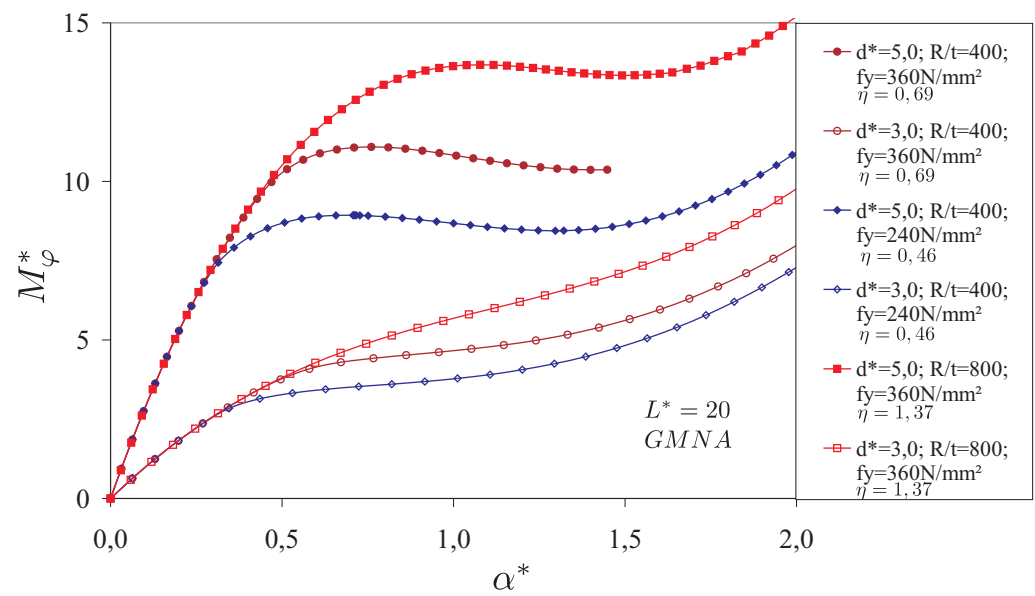

Abbildung 6.38: Momenten-Rotationskurven von Kreiszylinderschalen mit $L^{*}=20, d^{*}=3,0$ und $d^{*}=5,0$ mit unterschiedlichen $R / t$ und $f_{y}$

\subsubsection{Interaktion von geometrischer und materieller Nichtli- nearität}

Im Interaktionsdiagramm in Abbildung 6.39 ist der Quotient aus $M_{\varphi, c r i t, G M N A}^{*}$ und $M_{\varphi, c r i t, G N A}^{*}$ über dem Quotienten aus $M_{\varphi, c r i t, G M N A}^{*}$ und $\bar{M}_{\varphi, \max , M N A} \cdot \eta$ aufgetragen.

Der Einfluss der materiellen Nichtlinearität ist in dieser Darstellung auf der vertikalen Achse zu erkennen. Für $M_{\varphi, \text { crit }, G M N A}^{*} /\left(\bar{M}_{\varphi, \max , M N A} \cdot \eta\right)<0,25$ ist $M_{\varphi, c r i t, G M N A}^{*} / M_{\varphi, c r i t, G N A}^{*}=1,0$, d.h. die Kreiszylinderschalen liegen im elastischen Bereich. Für $M_{\varphi, c r i t, G M N A}^{*} /\left(\bar{M}_{\varphi, \max , M N A} \cdot \eta\right)>0,25$ macht sich der Einfluss aus materieller Nichtlinearität auf die kritischen Momente bemerkbar, und die Verhältniswerte auf der vertikalen Achse sinken. Dies ist der Fall bei Kreiszylinderschalen mit kleinen $R / t$-Verhältnissen und gleichzeitig niedrigen Streckgrenzen $f_{y}$. Mit sinkendem $\eta$ entlang der gestrichelten Geraden in Abbildung 6.39 nimmt der Einfluss der materiellen Nichtlinearität auf das kritische Moment zu. Gleichzeitig nimmt aber auch der Einfluss der geometrischen Nichtlinearität auf das kritische Moment ab, der auf der horizontalen Achse abgelesen werden kann. Der Einfluss der geometrischen Nichtlinearität ist umso kleiner, je weiter rechts sich die Verhältniswerte im Interaktionsdiagramm befinden, d.h. je 


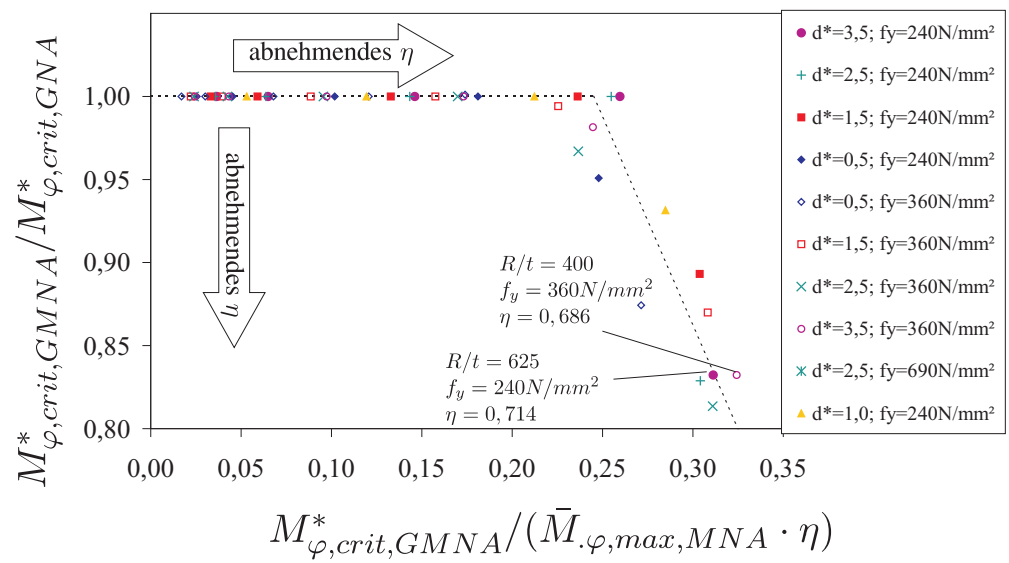

Abbildung 6.39: Interaktion von geometrischer und materieller Nichtlinearität für den Lastfall $M_{\varphi}$

kleiner das $R / t$-Verhältnis der Kreiszylinderschale bei konstantem $f_{y}$ ist.

In Abbildung 6.40 sind die Verhältniswerte $M_{\varphi, c r i t, G M N A}^{*} / M_{\varphi, c r i t, G N A}^{*}$ über $\eta$ aufgetragen. Für unterschiedliche $d^{*}$ ergeben sich nur geringe Unterschied in den Verhältniswerten. Für Kreiszylinderschalen mit $\eta>1,2$ bestehen keine Einflüsse aus plastischem Werkstoffverhalten. Die in Abbildung 6.40 eingezeichnete Kurve stellt dabei eine sichere Abschätzung der Verhältniswerte dar und kann mit

$$
M_{\varphi, c r i t, G M N A}^{*} / M_{\varphi, c r i t, G N A}^{*}=-0,56 \eta^{2}+1,45 \eta+0,07
$$

abgeschätzt werden.

Die normierten Last-Verformungskurven aus den Abbildungen 6.32 und 6.37 zeigen, dass sich Kreiszylinderschalen für Lastgrößen, die kleiner als $0,4 M_{\varphi, c r i t, G N A}^{*}$ sind, geometrisch linear verhalten. Unterhalb dieser Grenze sind die Kurven linear. Der plastische Bereich der Kreiszylinderschalen beginnt, wenn die Lastgrößen größer als $0,4 \bar{M}_{\varphi, \max , M N A}$ sind. 


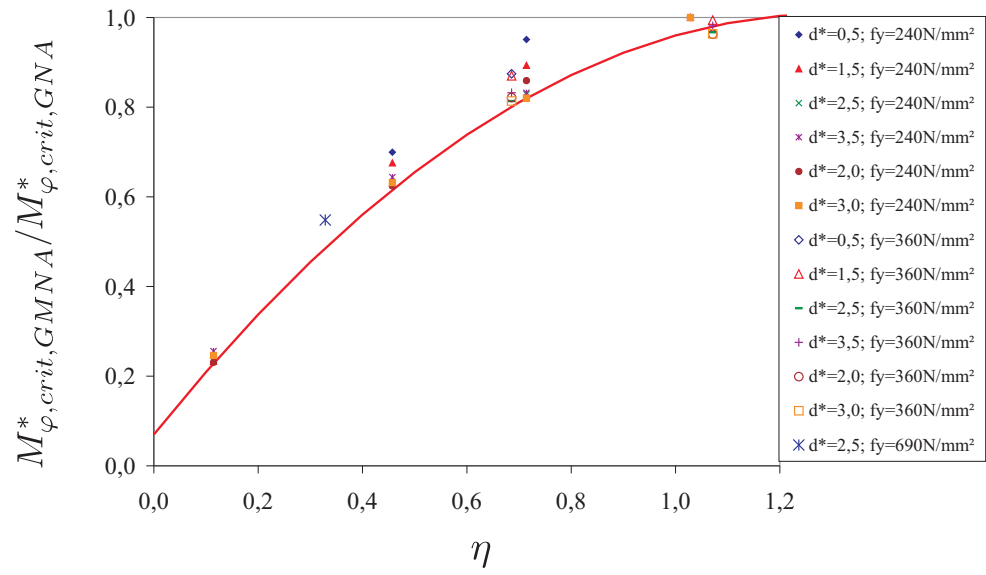

Abbildung 6.40: Einfluss der materiellen Nichtlinearität auf das kritische Moment $M_{\varphi, \text { crit }}^{*}$

\subsection{Kreiszylinderschalen unter Momentenbela- stung $M_{z}$ in Meridianrichtung}

\subsubsection{Allgemeines}

In diesem Abschnitt werden Kreiszylinderschalen untersucht, an deren Stutzenanschluss ein Moment $M_{z}$ in Meridianrichtung angreift. Das Moment kann in dimensionsloser Form mit

$$
M_{z}^{*}=P_{x}^{*} d^{*}=\frac{M_{z}}{E t^{3}} \sqrt{\frac{R}{t}}
$$

ausgedrückt werden.

Die Ergebnisse der geometrisch linearen Berechnungen mit elastischem Werkstoffverhalten $\left(M_{z} \mathrm{LA}\right)$ werden als dimensionslose Spannungsverläufe im Querschnitt der Kreiszylinderschale mit der Stutzenachse entlang des Meridians und des Umfangs dargestellt. Dabei wird die Bogenlänge $s_{\varphi}$ und die Längsachse $z$ durch $\sqrt{R t}$ dividiert. 
Die Spannungen werden in dimensionsloser Form mit

$$
\sigma_{M_{z}}^{*}=\sigma \frac{d_{r} t^{2}}{M_{z}}=\frac{\sigma}{E} \frac{d^{*}}{M_{z}^{*}} \frac{R}{t}
$$

dargestellt.

Die Steigung der Momenten-Rotationskurven im linearen Bereich wird in dimensionsloser Form als Anfangssteifigkeit

$$
c_{M_{z}}^{*}=\frac{M_{z}^{*}}{\alpha^{*}}=\frac{M_{z}}{\alpha} \frac{1}{E t^{3}}
$$

bezeichnet.

Die Ergebnisse der geometrisch nichtlinearen Berechnung ohne und mit Berücksichtigung des plastischen Werkstoffverhaltens $\left(M_{z}\right.$ GNA und $M_{z}$ GMNA) werden als Momenten-Rotationskurven dargestellt. Dabei wird das dimensionslose Moment $M_{z}^{*}$ über der dimensionslosen Verdrehung $\alpha^{*}$ aufgetragen.

Bei Berücksichtigung nur materieller Nichtlinearität ergibt sich das Moment $M_{z}$ in dimensionsloser Form aus

$$
\bar{M}_{z}=\bar{P}_{x} d^{*}=\frac{M_{z}}{f_{y} t^{2} \sqrt{R t}}
$$

\subsubsection{Geometrisch lineare Berechnungen mit elastischem Werkstoffgesetz $\left(M_{z} \mathbf{L A}\right)$}

In Abbildung 6.41 wurde der Verlauf der dimensionslosen Umfangsspannungen $\sigma_{\varphi}^{*}$ (a) und Längsspannungen $\sigma_{z}^{*}$ (b) entlang des Umfangs einer Kreiszylinderschale mit $L^{*}=20$ und $d^{*}=2,5$ aufgetragen. Die Kreiszylinderschale wurde dabei mit einem Einheitsmoment $M_{z}^{*}=1,0$ belastet. Da die Längs- und Umfangsspannungen entlang des Meridians um ein Vielfaches kleiner sind als die entsprechenden Spannungen entlang des Umfangs wurde auf eine Darstellung der Spannungen entlang des Meridians verzichtet. Es zeigt sich, dass die maximalen Spannungen $\sigma_{\varphi, \max , M_{z}}^{*}$ die Umfangsspannungen an der Außenseite der Kreiszylinderschalen sind. Da die Biegespannungen gegenüber den Membranspannungen dominieren, klingen die Spannungen entlang des Umfangs schnell ab. Der in Abbildung 6.41 dargestellte Verlauf der Spannungen ist typisch für Kreiszylinderschalen innerhalb des untersuchten Parameterbereichs. In den folgenden Untersuchungen werden nur die Umfangsspannungen $\sigma_{\varphi}^{*}$ und das Maxi$\operatorname{mum} \sigma_{\varphi, \max , M_{z}}^{*}$ betrachtet.

Zur Untersuchung der Einflüsse der Geometrieparameter $L^{*}, R / t$ und $d^{*}$ auf 

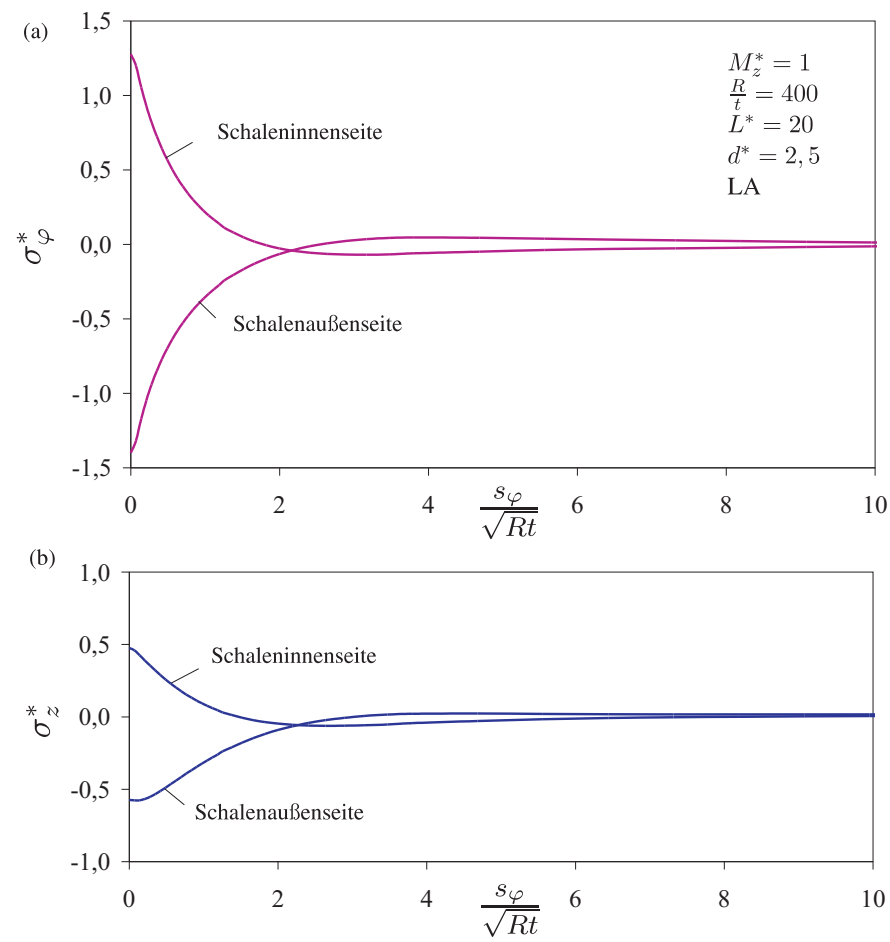

Abbildung 6.41: Verlauf von $\sigma_{\varphi}^{*}$ (a) und $\sigma_{z}^{*}(\mathrm{~b})$ entlang des Umfangs einer Kreiszylinderschale mit $R / t=400, L^{*}=20, d^{*}=2,5$ unter einer Belastung von $M_{z}^{*}=1,0$

\begin{tabular}{|c|c|c|}
\hline$R / t$ & $L^{*}$ & $d^{*}$ \\
\hline \hline 1000 & $10-80$ & 2,5 \\
\hline $400-5000$ & 20 & 2,5 \\
\hline 5000 & 80 & $0,5-3,5$ \\
\hline
\end{tabular}

Tabelle 6.9: Variationsparameter $\left(M_{z} \mathrm{LA}\right)$

den Spannungsverlauf der Kreiszylinderschale wird nach den in Tabelle 6.9 aufgeführten Schritten vorgegangen.

Zunächst wurde für eine Kreiszylinderschale mit $R / t=1000$ und $d^{*}=2,5$ die bezogenen Länge $L^{*}$ variiert. In Abbildung 6.42 ist der Verlauf der aus diesen 
Berechnungen resultierenden Umfangsspannungen entlang des Umfangs dargestellt. Dabei zeigte sich, dass $L^{*}$ keinen Einfluss auf den Spannungsverlauf hat und $L^{*}$ in den folgenden Untersuchungen beliebig innerhalb des Parameterbereichs gewählt werden kann.

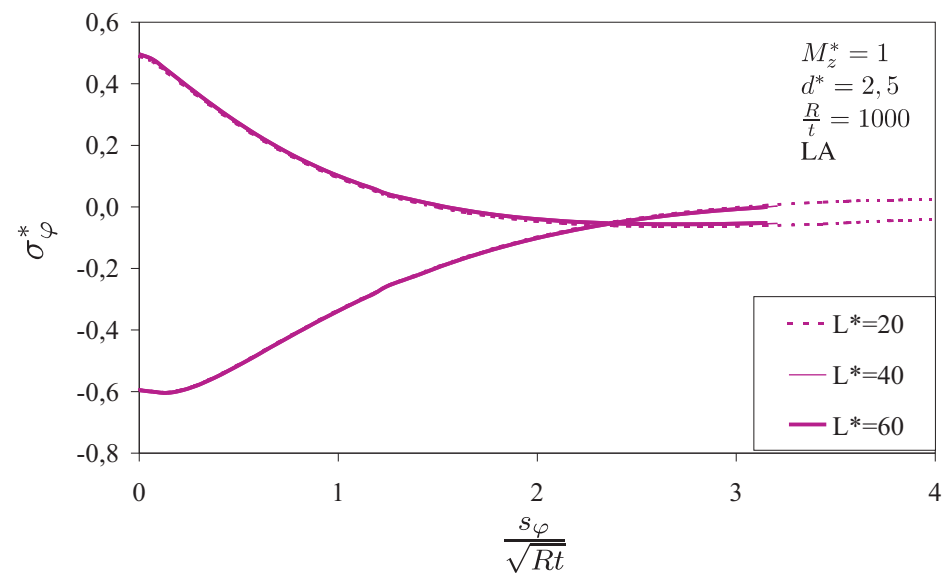

Abbildung 6.42: Verlauf von $\sigma_{\varphi}^{*}$ entlang des Umfangs einer Kreiszylinderschale mit $R / t=1000, d^{*}=2,5$ unter einer Belastung von $M_{z}^{*}=1,0$ für unterschiedliche $L^{*}$

Das $R / t$-Verhältnis wirkt sich dagegen geringfügig auf den Spannungsverlauf aus, wie Abbildung 6.43 am Beispiel einer Kreiszylinderschale mit $L^{*}=20$ und $d^{*}=2,5$ für unterschiedliche $R / t$-Verhältnisse verdeutlicht. Da die Auswirkungen unterschiedlicher $R / t$-Verhältnisse auf den Spannungsverlauf sehr klein sind, wird bei den weiteren Berechnungen auf eine Diffenzierung unterschiedlicher $R / t$-Verhältnisse verzichtet. Auf der sicheren Seite liegend werden Kreiszylinderschalen mit $R / t=5000$ untersucht, die auch den Bereich kleinerer $R / t$-Verhältnisse abdecken.

Im nächsten Schritt wurde der Einfluss der bezogenen Stutzendurchmesser $d^{*}$ auf den Spannungsverlauf untersucht. Dabei zeigte sich, dass $d^{*}$ einen kleinen aber nicht zu vernachlässigenden Einfluss auf die Spannungsmaxima $\sigma_{\varphi, \max , M_{z}}^{*}$ hat, wie aus Abbildung 6.44 hervorgeht. Dabei wurde die Kreiszylinderschale mit dem ungünstigsten $R / t$-Verhältnis $(R / t=5000)$ und $L^{*}=20$ betrachtet. 
6 Numerische Serienberechnungen von Kreiszylinderschalen unter lokaler

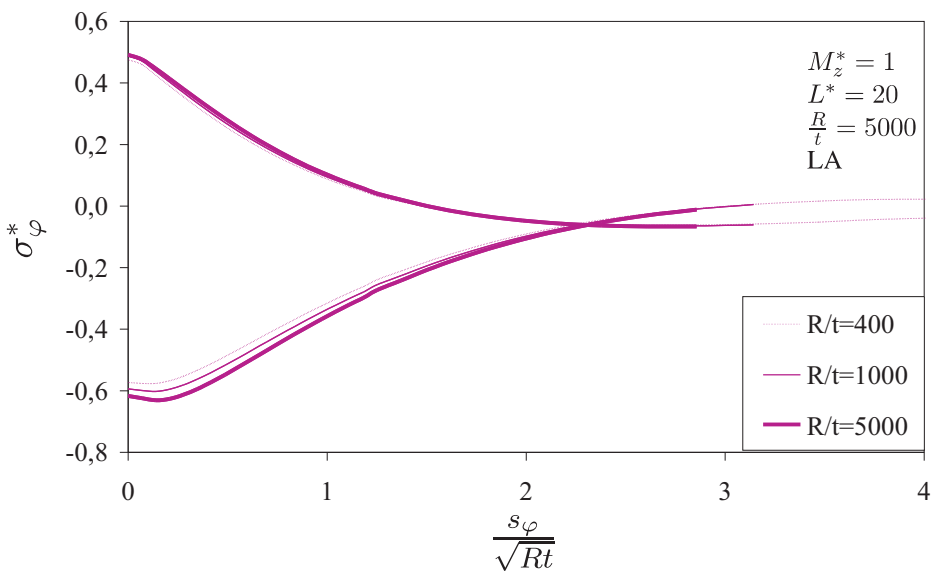

Abbildung 6.43: Verlauf von $\sigma_{\varphi}^{*}$ entlang des Umfangs einer Kreiszylinderschale mit $L^{*}=20, d^{*}=2,5$ unter einer Belastung von $M_{z}^{*}=1,0$ für unterschiedliche $R / t$

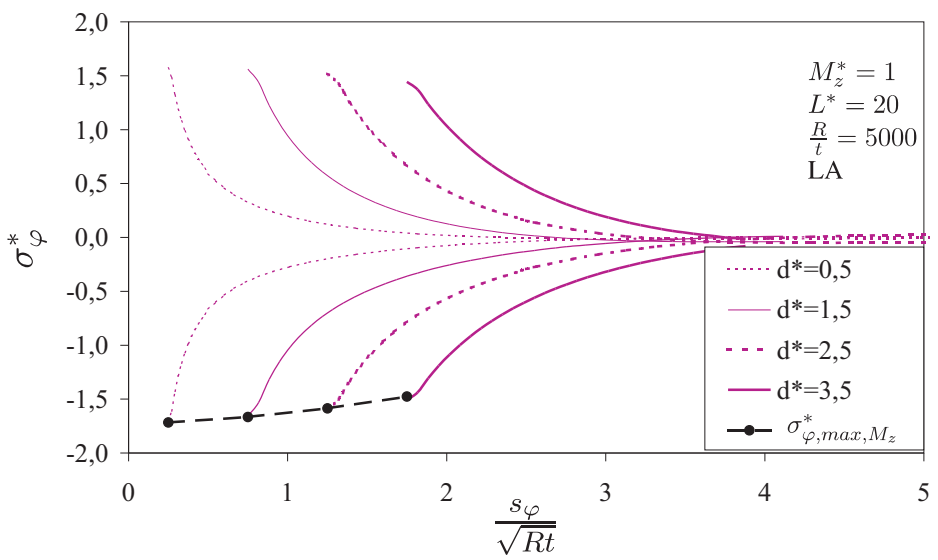

Abbildung 6.44: Verlauf von $\sigma_{\varphi}^{*}$ entlang des Umfangs einer Kreiszylinderschale mit $R / t=5000, L^{*}=20$ unter einer Belastung von $M_{z}^{*}=1,0$ für unterschiedliche $L^{*}$ 
Die maximale Spannung $\sigma_{\varphi, \max , M_{z}}^{*}$ wird mit zunehmendem $d^{*}$ geringer und kann in Abhängigkeit von $d^{*}$ mit

$$
\sigma_{\varphi, \max , M_{z}}^{*}=0,015\left(d^{*}\right)^{2}+0,02 d^{*}-1,73
$$

abgeschätzt werden.

Die mit WRCB 297 [29] ermittelten maximalen Spannungen am Stutzenanschluss unter einem Einheitsmoment $M_{z}^{*}=1$ wurden in Abbildung 6.45 über $d^{*}$ aufgetragen. Bei der Spannungsermittlung mit [29] wurde von einem steifen Stutzen mit einer Wanddicke $t_{r} \geq t$ ausgegangen. Dabei ist in Abbildung 6.45 auch das in [29] dokumentiert Ergebniss einer FE-Berechnung aufgeführt. Der Vergleich der numerisch ermittelten Spannungen, die mit Gleichung 6.24 abgeschätzt wurden, zeigt, dass diese Spannungen mit dem FE-Ergebnis aus [29] sehr gut übereinstimmen. Die Spannungen, die sich nach der analytischen Lösung in [29] ergeben, liegen dagegen rund $10 \%$ über den numerischen Ergebnissen.

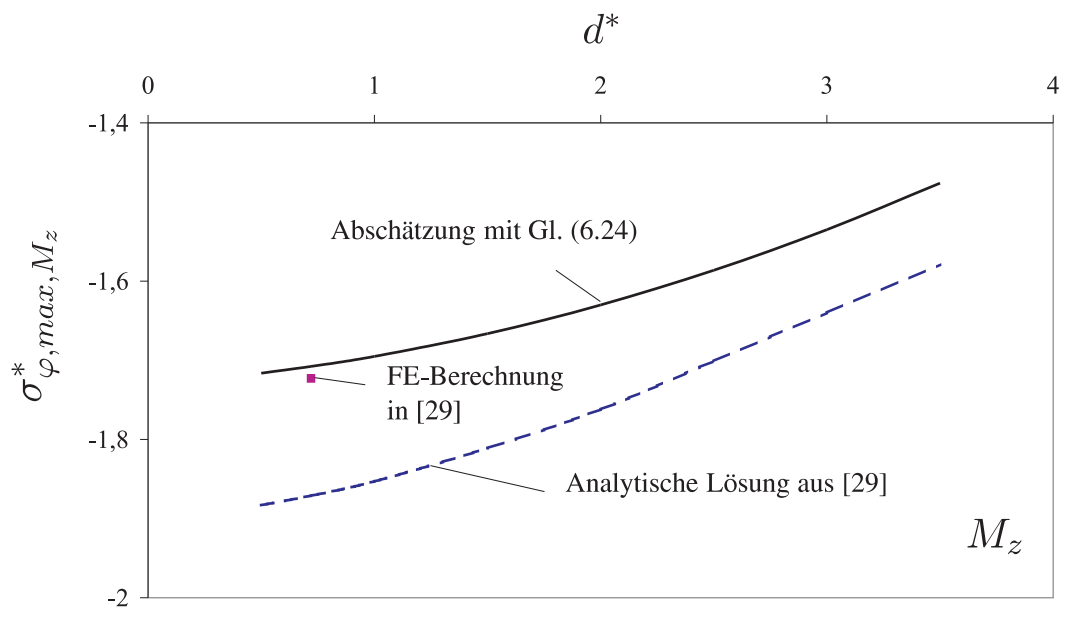

Abbildung 6.45: Maximale Umfangsspannungen nach Gleichung 6.24 und WRCB 297 [29] 


\subsubsection{Geometrisch nichtlineare Berechnungen mit elastischem Werkstoffgesetz $\left(M_{z}\right.$ GNA)}

Die Untersuchung der Einflüsse der Parameter $L^{*}, R / t$ und $d^{*}$ auf das Tragverhalten der Kreiszylinderschale erfolgte anhand des in Tabelle 6.10 aufgeführten Variationsschemas.

\begin{tabular}{|c|c|c|}
\hline$R / t$ & $L^{*}$ & $d^{*}$ \\
\hline \hline $400-5000$ & 40 & 2,5 \\
\hline 1000 & $10-80$ & 2,5 \\
\hline 400 & 20 & $0,5-3,5$ \\
\hline
\end{tabular}

Tabelle 6.10: Variationsparameter $\left(M_{z} \mathrm{GNA}\right)$

Zunächst wurde der Einfluss des $R / t$-Verhältnisses auf das Tragverhalten untersucht. Wie in Abbildung 6.46 am Beispiel einer Kreiszylinderschale mit $L^{*}=40$ und $d^{*}=2,5$ gezeigt wird, haben unterschiedliche $R / t$-Verhältnisse keine Auswirkungen auf den Verlauf der Momenten-Rotationskurve im Anfangsbereich. Mit zunehmender Verdrehung $\alpha^{*}$ weichen die Kurven vom gemeinsamen Verlauf ab.

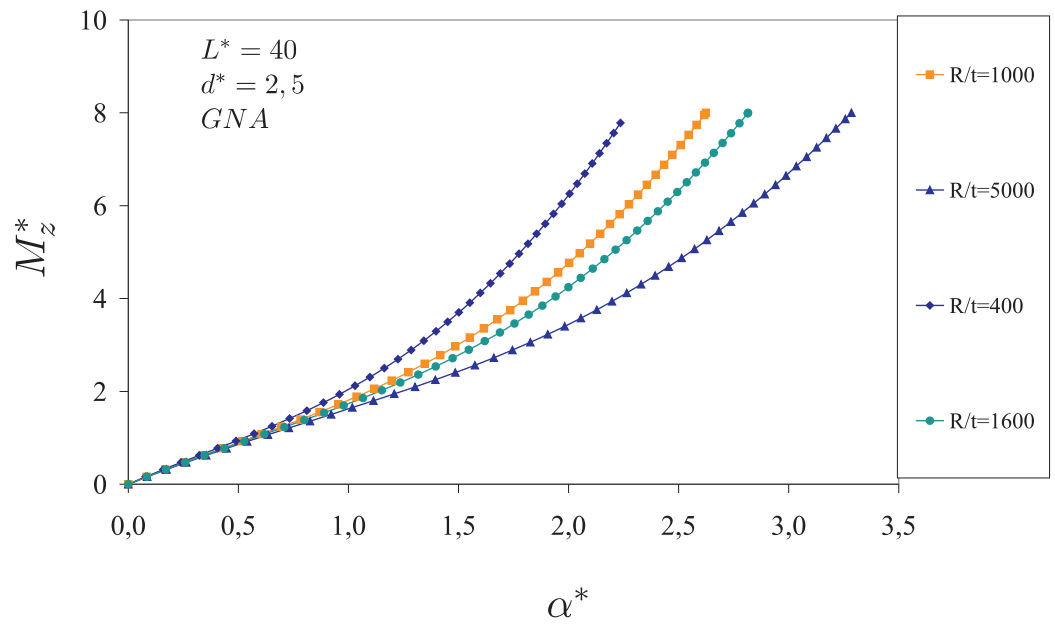

Abbildung 6.46: Momenten-Rotationskurven von Kreiszylinderschalen mit $L^{*}=40, d^{*}=2,5$ und $400 \leq R / t \leq 5000$ 
Im nächsten Schritt wurde der Einfluss des bezogenen Randabstandes $L^{*}$ auf das Tragverhalten der Kreiszylinderschale untersucht. Wie in Abbildung 6.47 am Beispiel einer Kreiszylinderschale mit $R / t=1000$ und $d^{*}=2,5$ gezeigt wird, haben unterschiedliche bezogene Randabstände $L^{*}$ durchaus einen Einfluss auf das Tragverhalten im Anfangsbereich. Die Steigung $c_{M_{z}}^{*}$ der Kurven im linearen Bereich ist abhängig von $L^{*}$.

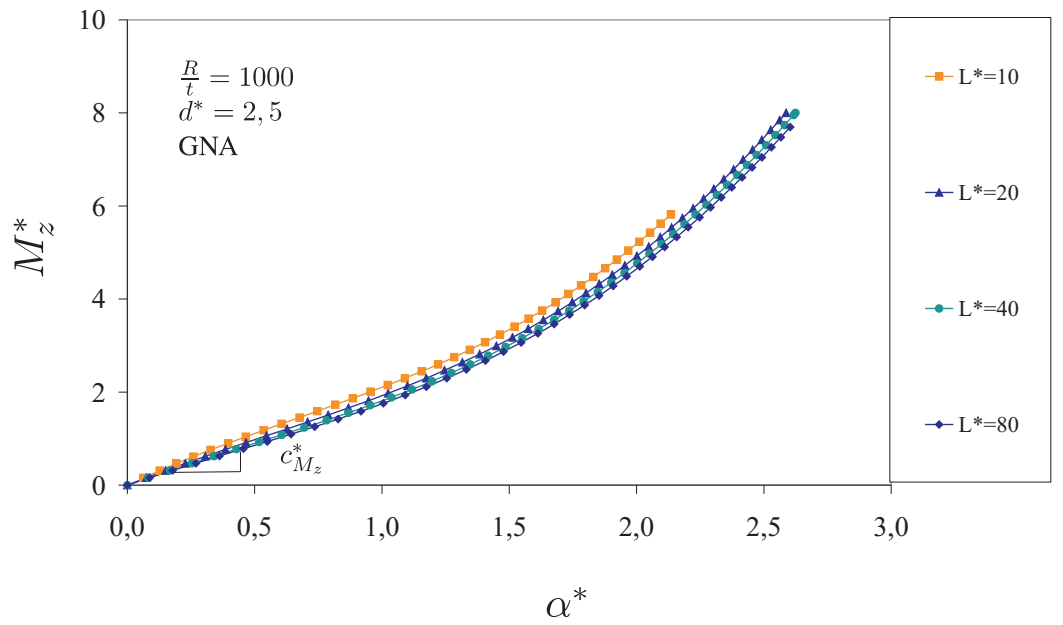

Abbildung 6.47: Momenten-Rotationskurven von Kreiszylinderschalen mit $R / t=1000, d^{*}=25$ und $20 \leq L^{*} \leq 60$

Im letzten Schritt wurde bei konstantem $L^{*}$ und $R / t$ der bezogene Stutzendurchmesser $d^{*}$ variiert. Wie in Abbildung 6.48 am Beispiel einer Kreiszylinderschale mit $L^{*}=20$ und $R / t=400$ mit $d^{*}$ zwischen 0,5 und 3,5 deutlich zu erkennen ist, nimmt die Steigung der Momenten-Rotationskurven mit zunehmendem $d^{*} \mathrm{zu}$.

Bei diesem Lastfall sind die Membrananteile gegenüber den Biegeanteilen äußerst klein, so dass auch bei sehr großen bezogenen Stutzendurchmessern $d^{*}$ kein Stabilitätsproblem auftritt. Daher gilt es, ein Abgrenzungskriterium zu finden, das nicht vollkommen willkürlich gewählt ist. In Anlehnung an Abschnitt 6.5.3 und Abschnitt 6.3 wird daher als dimensionslose Grenzverdrehung $\alpha^{*}$ ein Wert von 1,0 gewählt. Damit ist der Zusammnenhang zwischen dem LastVerformungsverhalten der Kreiszylinderschalen im Lastfall $M_{z}$ und dem verformungskritischen Zustand beim Auftreten eines Stabilitätsproblems bei anderen Lastfällen gegeben. Die kritischen Momente werden in diesen Fällen stets zur sicheren Seite hin abgeschätzt. Fallen die normierten Momenten-Rotationskurven 


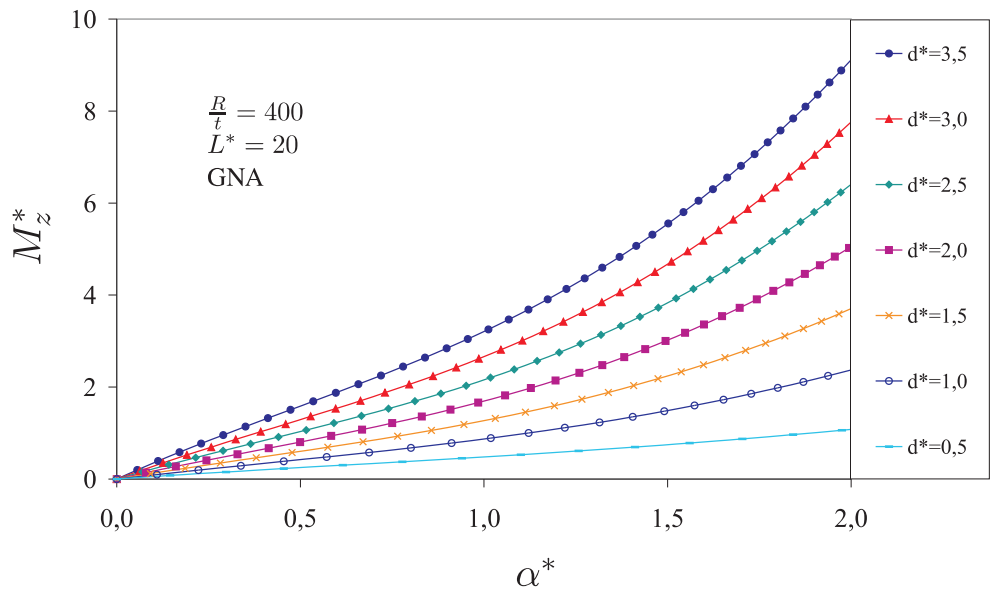

Abbildung 6.48: Momenten-Rotationskurven von Kreiszylinderschalen mit $L^{*}=20, R / t=400$ und $0,5 \leq d^{*} \leq 3,5$

des Lastfalls $M_{z}$ mit den Kurven des Lastfalls $M_{\varphi}$ zusammen, so entspricht die Normierungsgröße dem kritischen Moment bei einer Verdrehung $\alpha^{*}$ von etwa 1,0 (vgl. Abschnitt 6.3).

In Abbildung 6.49 sind die normierten Momenten-Rotationskurven einer Kreiszylinderschale mit $R / t=400, L^{*}=20$ für $0,5 \leq d^{*} \leq 3,5$ für den Lastfall $M_{z}$ und für $d^{*}=5,0$ für den Lastfall $M_{\varphi}$ dargestellt. Da die Bestimmung des kritischen Momentes ausschließlich über die Anfangssteifigkeit erfolgt, hat das $R / t$-Verhältnis keinen Einfluss auf $M_{z, c r i t, G N A}^{*}$. Wie bereits gezeigt wurde, wirkt sich $R / t$ nur auf den Bereich sehr großer Verfomungen aus. Der bezogene Randabstand $L^{*}$ beeinflusst dagegen das kritische Moment. $M_{z, c r i t, G N A}^{*}$ ist in Abhängigkeit von $L^{*}$ und $d^{*}$ in Abbildung 6.50 dargestellt. Dabei wird deutlich, dass mit zunehmendem $d^{*}$ auch der Einfluss von $L^{*}$ auf das kritische Moment $M_{z, c r i t, G N A}^{*}$ zunimmt. Die Anfangssteifigkeit $c_{M_{z}}^{*}$ der MomentenRotationskurven ist in Abbildung 6.51 über $L^{*}$ für unterschiedliche $d^{*}$ aufgetragen. 
6 Numerische Serienberechnungen von Kreiszylinderschalen unter lokaler

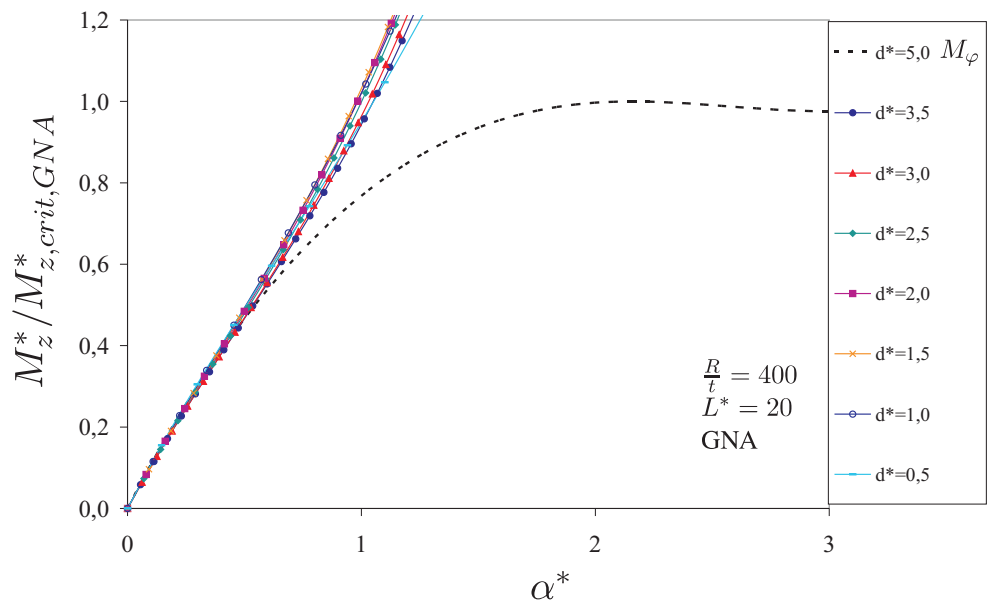

Abbildung 6.49: Normierte Momenten-Rotationskurven für den Lastfall $M_{z}$ mit $0,5 \leq d^{*} \leq 3,5$ und den Lastfall $M_{\varphi}$ für $d^{*}=5,0$

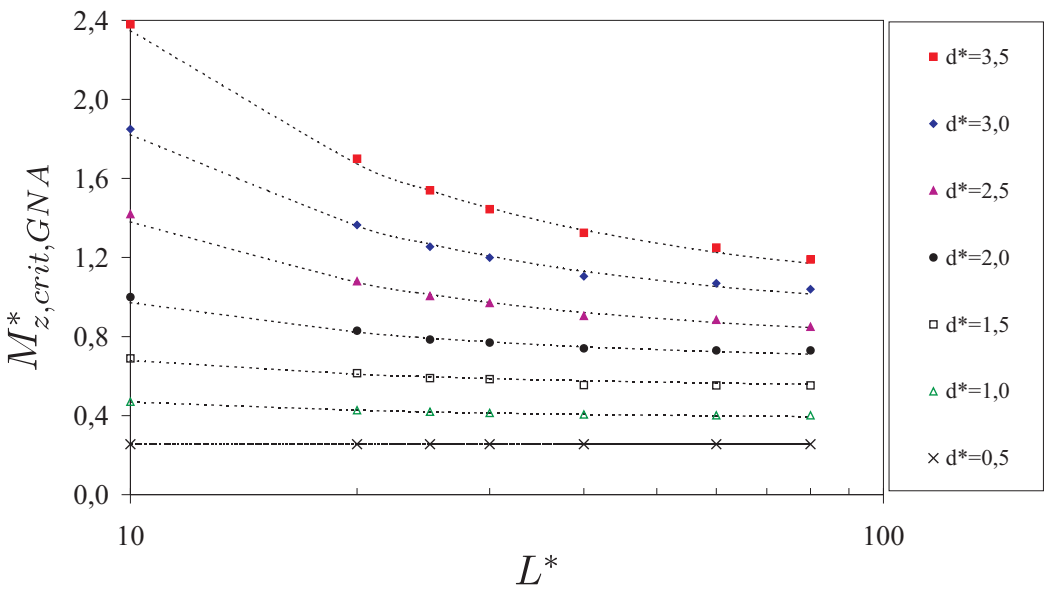

Abbildung 6.50: $M_{z, c r i t, G N A}^{*}$ in Abhängigkeit von $L^{*}$ und $d^{*}$ 


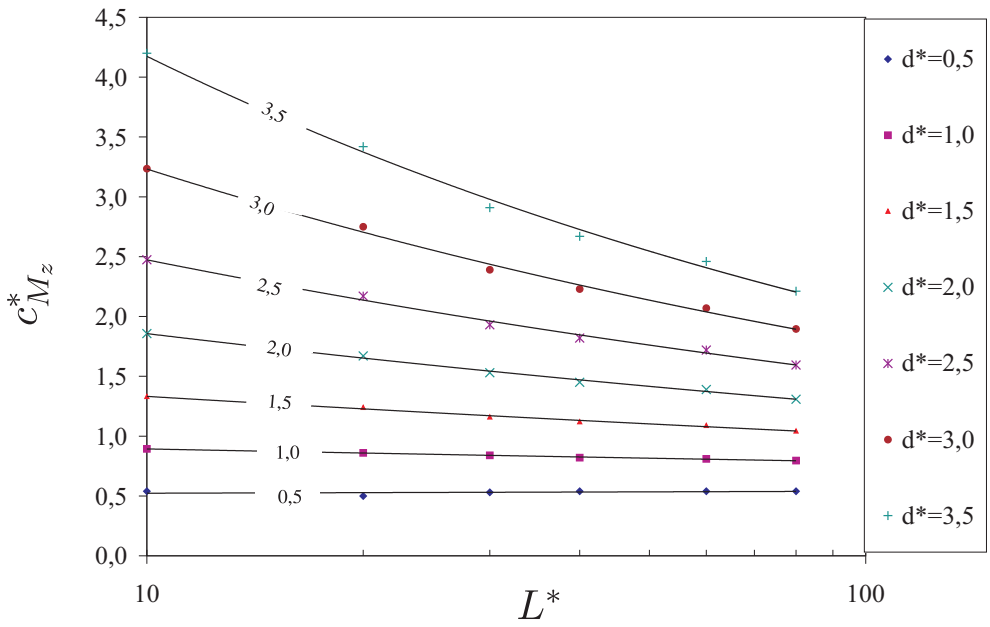

Abbildung 6.51: Anfangssteifigkeit $c_{M_{z}}^{*}$ in Abhängigkeit von $L^{*}$ und $d^{*}$

\subsubsection{Geometrisch lineare Berechnung mit plastischem Werk- stoffgesetz $\left(M_{z}\right.$ MNA)}

Den Berechnungen liegt das Werkstoffgesetz nach Abschnitt 3.5 zugrunde. In den Parameterstudien wurden die in Tabelle 6.11 aufgeführten Werte variiert.

\begin{tabular}{|c|c|c|c|}
\hline$R / t$ & $L^{*}$ & $d^{*}$ & $f_{y}$ \\
\hline \hline 1000 & $20-80$ & 2,5 & 240 \\
\hline 400 & 20 & 2,5 & $240-690$ \\
\hline $400-5000$ & 20 & 2,5 & 360 \\
\hline 1600 & 40 & $0,5-3,5$ & 240 \\
\hline
\end{tabular}

Tabelle 6.11: Variationsparameter $\left(M_{z} \mathrm{MNA}\right)$

Zunächst wurde der bezogene Randabstand $L^{*}$ bei konstantem $d^{*}, R / t$ und $f_{y}$ varriert. Wie Abbildung 6.52 am Beispiel einer Kreiszylinderschale mit $R / t=400, d^{*}=2,5, f_{y}=360 \mathrm{~N} / \mathrm{mm}^{2}$ und unterschiedlichen $L^{*}$ zeigt, nähern sich die Kurven asymptotisch einem Grenzwert $\bar{M}_{z, \max , M N A}$, der umso größer ist, je größer $L^{*}$ ist. Da dieser Grenzwert nur näherungsweise bestimmt werden 
kann und die Unterschiede bei den Grenzmomenten, die sich für unterschiedliche $L^{*}$ ergeben, sehr gering sind, wird der Einfluss von $L^{*}$ auf das Grenzmoment vernachlässigt. Die Steigungen der Momenten-Rotationskurven sind für gleiche $R / t, f_{y}$ und $d^{*}$ vollkommen unabhängig von $L^{*}$. Da das Moment $M_{z}$ mit $f_{y}$ und $t^{2}$ normiert wurde, ergibt sich die Steigung der Momenten-Rotationskurven zu $c_{M_{z}}^{*} / \eta$.

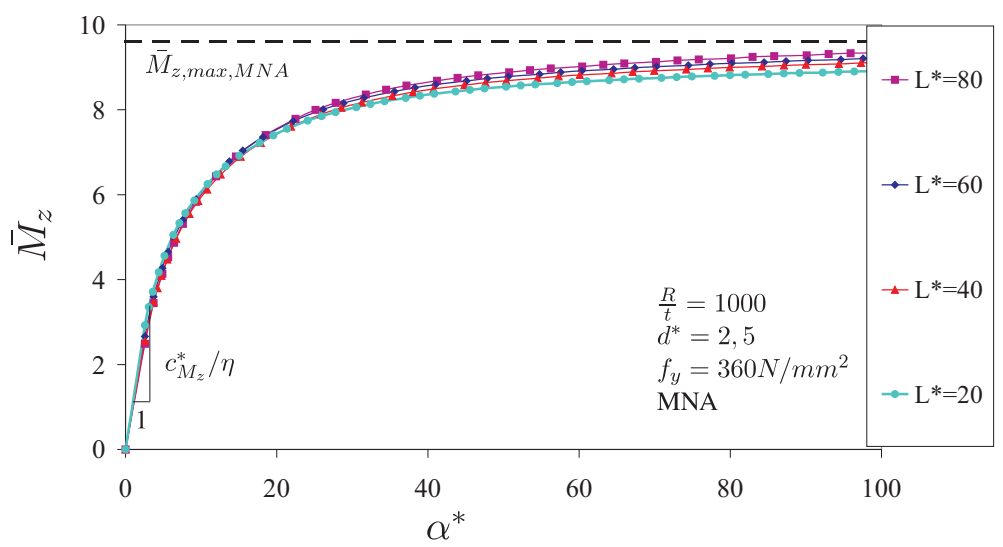

Abbildung 6.52: Momenten-Rotationskurven von Kreiszylinderschalen mit $R / t=400, d^{*}=2,5, f_{y}=360 \mathrm{~N} / \mathrm{mm}^{2}$ und variablen bezogenen Randabständen $L^{*}$

In Abbildung 6.53 sind die Momenten-Rotationskurven einer Kreiszylinderschale mit $L^{*}=20, d^{*}=2,5$ und veränderlichem $R / t$-Verhältnis sowie veränderlicher Steckgrenze $f_{y}$ aufgetragen. Dabei wird deutlich, dass sich diese Kurven vollkommen unabhängig von $f_{y}$ und $R / t$ dem Grenzwert $\bar{M}_{z, \max , M N A}$ nähern. Die Steigungen der Kurven, d.h. die Steifigkeit der Kreiszylinderschale wird dagegen von $R / t$ und folglich $\eta$ beinflusst.

Im letzten Schritt wurden die bezogenen Stutzendurchmesser $d^{*}$ variiert. Die Momenten-Rotationskurven, die sich aus diesen Berechnungen ergeben, sind in Abbildung 6.54 für eine Kreiszylinderschale mit $L^{*}=40, R / t=1600$ und einer Streckgrenze $f_{y}=240 \mathrm{~N} / \mathrm{mm}^{2}$ für unterschiedliche $d^{*}$ dargestellt. Dabei zeigt sich, dass sich die Kurven in Abhängigkeit von $d^{*}$ asymptotisch den Grenzwerten $\bar{M}_{z, \max , M N A}$ nähern. Dieser Grenzwert ist umso größer, je größer $d^{*}$ ist. Das Grenzmoment $\bar{M}_{z, \max , M N A}$ in Abhängigkeit von $d^{*}$ ist in Abbildung 6.55 dar- 


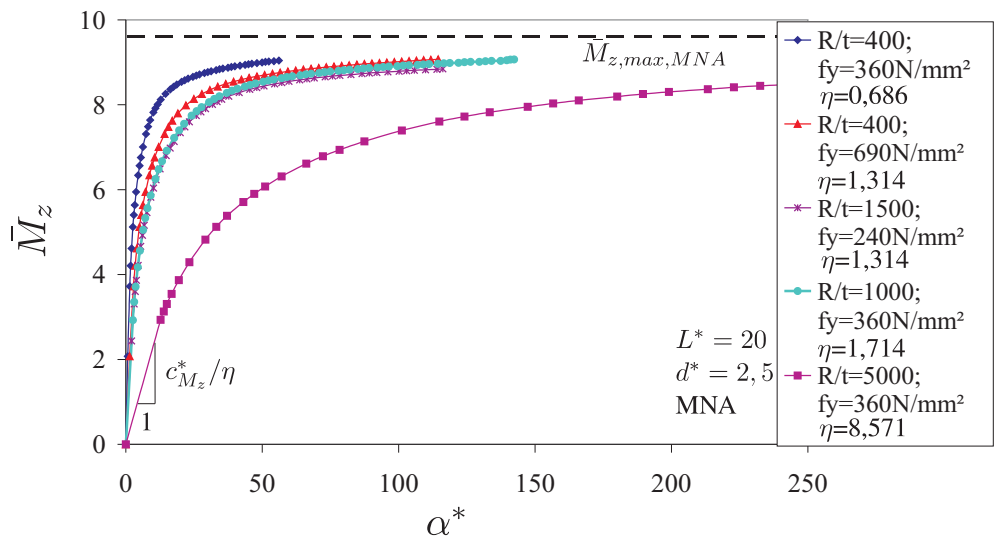

Abbildung 6.53: Momenten-Rotationskurven von Kreiszylinderschalen mit $L^{*}=20, d^{*}=2,5$ und variablen $R / t$ und $f_{y}$

gestellt und kann mit

$$
\bar{M}_{z, \max , M N A}=0,71\left(d^{*}\right)^{2}+1,94 d^{*}+0,5
$$

abgeschätzt werden.

Die mit dem Grenzmoment $\bar{M}_{z, \max , M N A}$ normierten MomentenRotationskurven sind in Abbildung 6.56 dargestellt. 
6 Numerische Serienberechnungen von Kreiszylinderschalen unter lokaler

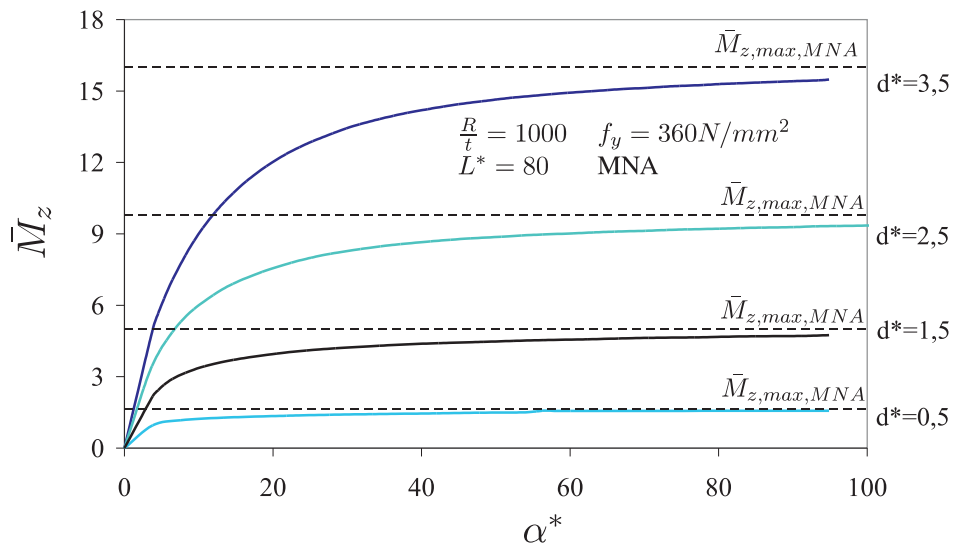

Abbildung 6.54: Momenten-Rotationskurven von Kreiszylinderschalen mit $L^{*}=20, R / t=400, f_{y}=240 \mathrm{~N} / \mathrm{mm}^{2}$ und bezogenen Stutzendurchmessern $d^{*}$ zwischen 0,5 und 3,5.

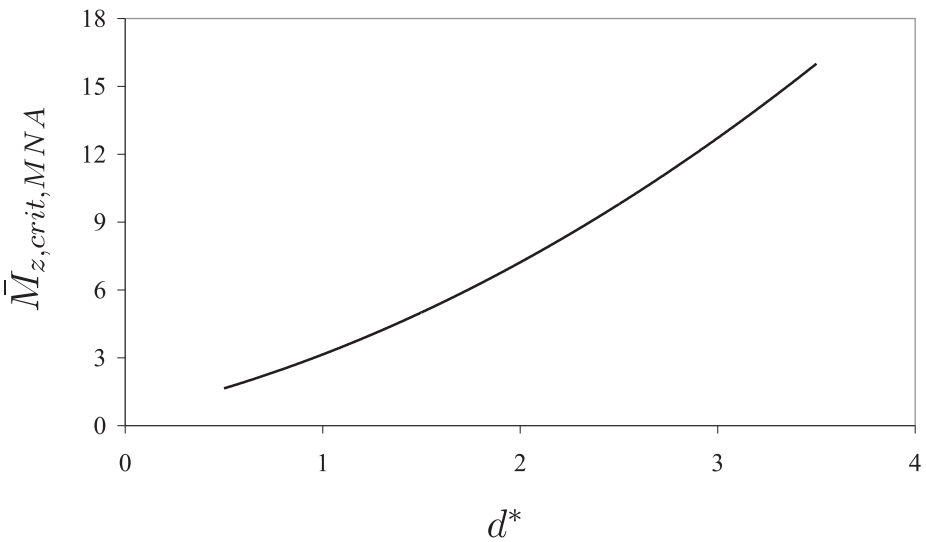

Abbildung 6.55: Kritisches Moment $\bar{M}_{z, \max , M N A}$ in Abhängigkeit von $d^{*}$ 
6 Numerische Serienberechnungen von Kreiszylinderschalen unter lokaler

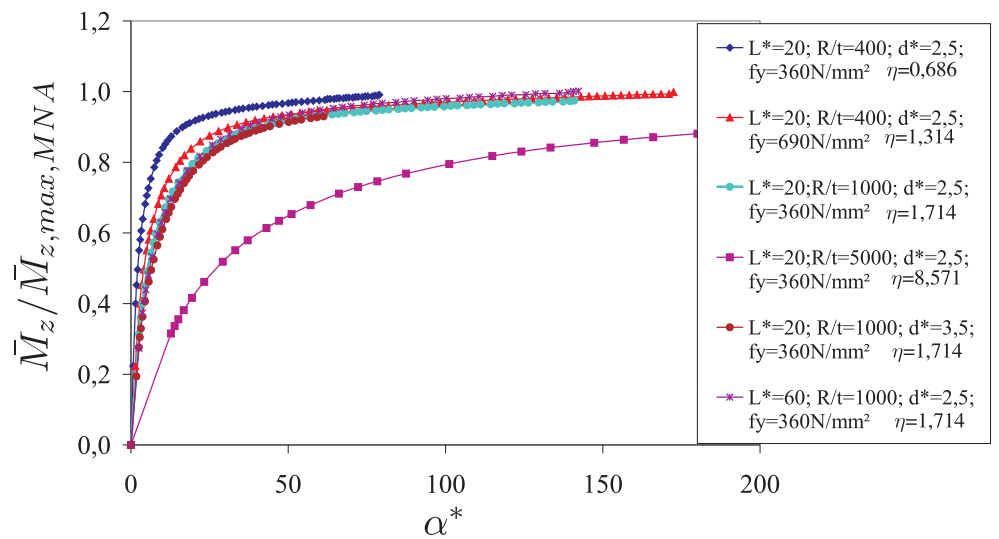

Abbildung 6.56: Normierte Momenten-Rotationskurven von Kreiszylinderschalen mit $L^{*}=20, R / t=400, f_{y}=240 \mathrm{~N} / \mathrm{mm}^{2}$ und bezogenen Stutzendurchmessern $d^{*}$ zwischen 0,5 und 3,5.

\subsubsection{Geometrisch nichtlineare Berechnung mit plastischem Werkstoffgesetz $\left(M_{z}\right.$ GMNA)}

Bei Berücksichtigung der geometrischen und materiellen Nichtlinearität kann das Tragverhalten der Kreiszylinderschale von $L^{*}, R / t, d^{*}$ und von der Streckgrenze $f_{y}$ beeinflusst werden. Der Einfluss dieser Parameter wird durch Variation der in Tabelle 6.12 aufgeführten Werte untersucht. Für eine Kreiszylinderschale mit

\begin{tabular}{|c|c|c|c|}
\hline$R / t$ & $L^{*}$ & $d^{*}$ & $f_{y}$ \\
\hline \hline 400 & $20-80$ & 2,5 & 240 \\
\hline 400 & 20 & 2,5 & $240-690$ \\
\hline $400-5000$ & 20 & 2,5 & 240 \\
\hline
\end{tabular}

Tabelle 6.12: Variationsparameter $\left(M_{z} \mathrm{GMNA}\right)$

veränderlichem $L^{*}$ bei konstantem $d^{*}=2,5, R / t=400$ und $f_{y}=240 \mathrm{~N} / \mathrm{mm}^{2}$ ergeben sich die in Abbildung 6.57 dargestellten Momenten-Rotationskurven. Dabei wird deutlich, dass das Tragverhalten der Kreiszylinderschale in sehr geringem Maße von $L^{*}$ beeinflusst wird. 
6 Numerische Serienberechnungen von Kreiszylinderschalen unter lokaler

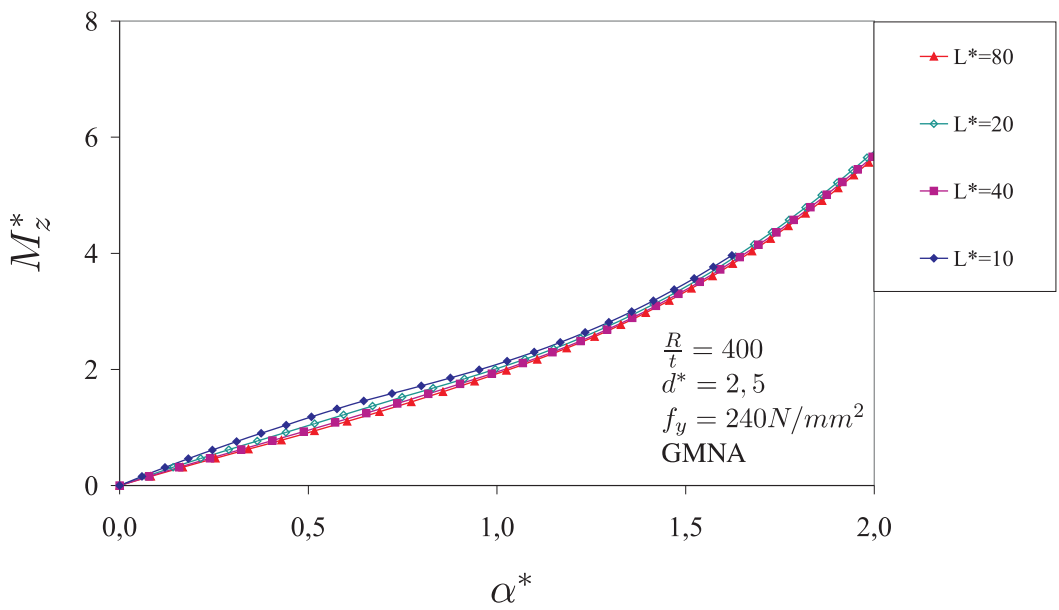

Abbildung 6.57: Momenten-Rotationskurven von Kreiszylinderschalen mit $R / t=400, d^{*}=2,5, f_{y}=240 \mathrm{~N} / \mathrm{mm}^{2}$ mit unterschiedlichen $L^{*}$

Im nächsten Schritt wurden bei konstantem $L^{*}=20$ und $d^{*}=2,5$ die $R / t$-Verhältnisse und die Streckgrenze $f_{y}$ der Kreiszylinderschalen variiert. Wie Abbildung 6.58 zeigt, unterscheiden sich die Kurven bei konstantem $L^{*}$ und konstantem $d^{*}$ nur im Bereich sehr großer Verformungen. Im linearen Anfangsbereich sind die Kurvenverläufe und damit die Steigungen identisch.

\subsubsection{Interaktion von geometrischer und materieller Nichtli- nearität}

Analog zu den Lastfällen $P_{x}$ und $M_{\varphi}$ kann aus den normierten LastVerformungskurven aus den Abbildungen 6.49 und 6.56 die Grenze zwischen linearem und nichtlinearem Bereich abgelesen werden. Der geometrisch lineare Bereich ist folglich auf Lastgrößen, die kleiner als $0,4 M_{z, c r i t, G N A}^{*}$ sind, beschränkt. Der elastische Bereich endet bei $0,4 \bar{M}_{z, \max , M N A}$. 
6 Numerische Serienberechnungen von Kreiszylinderschalen unter lokaler

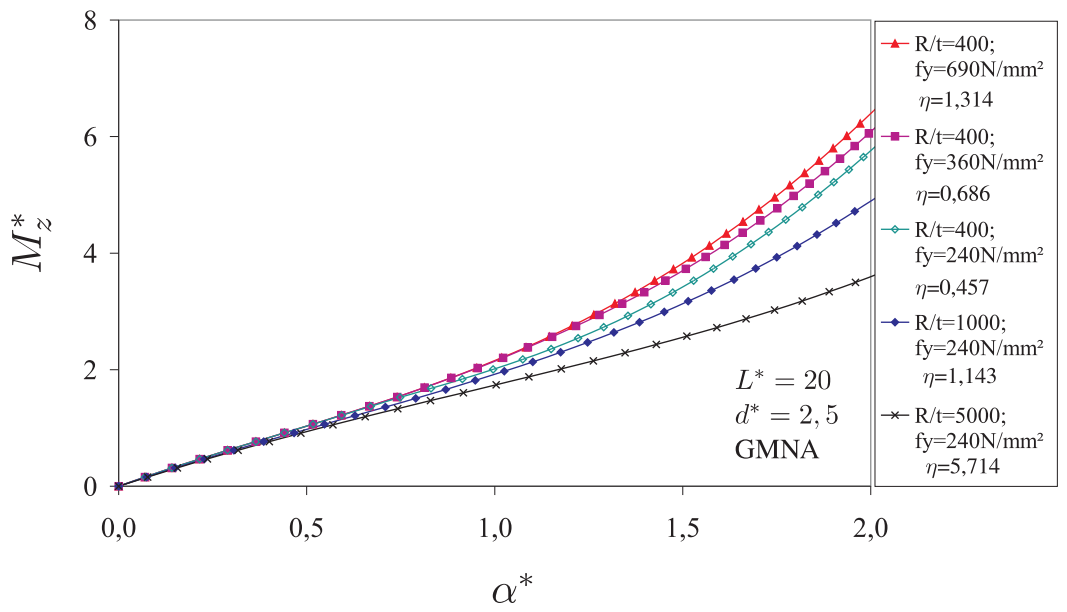

Abbildung 6.58: Momenten-Rotationskurven von Kreiszylinderschalen mit $L^{*}=20, d^{*}=2,5$ mit unterschiedlichen $R / t$ und $f_{y}$

\subsection{Zusammenfassung der Ergebnisse}

In den Untersuchungen an Kreiszylinderschalen, die durch ein radiale Einzellasten oder durch Momente in Umfangs- oder Meridianrichtung beansprucht werden, wurde der Einfluss des $R / t$-Verhältnisses, des bezogenen Randabstandes $L^{*}$, des bezogenen Stutzendurchmessers $d^{*}$ sowie der Streckgrenze $f_{y}$ auf das Tragverhalten analysiert. Das Tragverhalten von Kreiszylinderschalen, die durch eine radiale Einzellast $P_{x}$ beansprucht werden, wird vom bezogenen Randabstand $L^{*}$ und vom bezogenen Stutzendurchmesser $d^{*}$ beeinflusst. Bei einer Belastung durch ein Moment $M_{\varphi}$ in Umfangsrichtung beeinflusst im wesentlichen der bezogene Stutzendurchmesser $d^{*}$ das Tragverhalten der Kreiszylinderschale. Bei beiden Lastfällen nimmt die Tragfähigkeit der Kreiszylinderschale in Abhängigkeit vom $R / t$-Verhältnis der Kreiszylinderschale und der Streckgrenze des Werkstoffes - ausgedrückt durch den Plastizitätsfaktor $\eta$ - ab. Das Tragverhalten von Kreiszylinderschalen, die durch ein Moment $M_{z}$ in Längsrichtung beansprucht werden, wird maßgeblich von $L^{*}$ und $d^{*}$ beeinflusst. Die Abminderung der Tragfähigkeit infolge des plastischen Werkstoffverhaltens zeigt sich jedoch erst im Bereich großer Verformungen. 


\section{Verstärkung der Kreiszylinderschale im Stutzenbereich}

\subsection{Varianten der Verstärkungsmaßnahmen}

\subsubsection{Hintergrund}

Lokale Lasten, die durch den Stutzen in die Kreiszylinderschale eingeleitet werden, führen im Stutzenanschlussbereich zu großen Spannungen im Mantelblech. Wie die Untersuchungen zum Spannungsverlauf der unverstärkten Kreiszylinderschale für verschiedene Lastfälle in den Abschnitten 6.4.2, 6.5.2 und 6.6.2 gezeigt haben, sind die großen Spannungen lokal begrenzt, da die Biegespannungen gegenüber den Membranspannungen überwiegen und schnell abklingen. Zur Reduktion der großen Spannungen kann das Mantelblech der Kreiszylinderschale im Bereich des Stutzenanschlusses verstärkt werden. Für die Untersuchungen des Einflusses dieser Verstärkung des Mantelbleches auf das Tragverhalten der Kreiszylinderschale werden im Rahmen dieser Arbeit nur kreisrunde scheibenförmige Verstärkungen nach Abbildung 7.1 betrachtet, da für die in der Praxis üblicherweise ausgeführten rechteckigen Bleche der Rechenaufwand erheblich größer und das Verhalten nicht grundsätzlich anders ist. Dabei wird unterschieden zwischen auf das Mantelblech aufgesetzten und in das Mantelblech eingesetzten Verstärkungsblechen.

\subsubsection{Aufgesetzte Verstärkungsbleche}

Als scheibenförmige Verstärkung des Mantelbleches werden häufig Bleche in der Nähe des Stutzens auf das Mantelblech aufgesetzt. Der Schnitt durch den Stutzenanschluss einer Kreiszylinderschale, die durch ein aufgesetztes Blech mit der Dicke $t_{v}$ und einem Durchmesser $d_{v}$ verstärkt wird, ist beispielhaft in Abbildung 7.2 (a) dargestellt. Dabei wird das Verstärkungsblech am inneren Rand an den Stutzen und am äußeren Rand auf das Mantelblech geschweißt. Bei geometrisch 


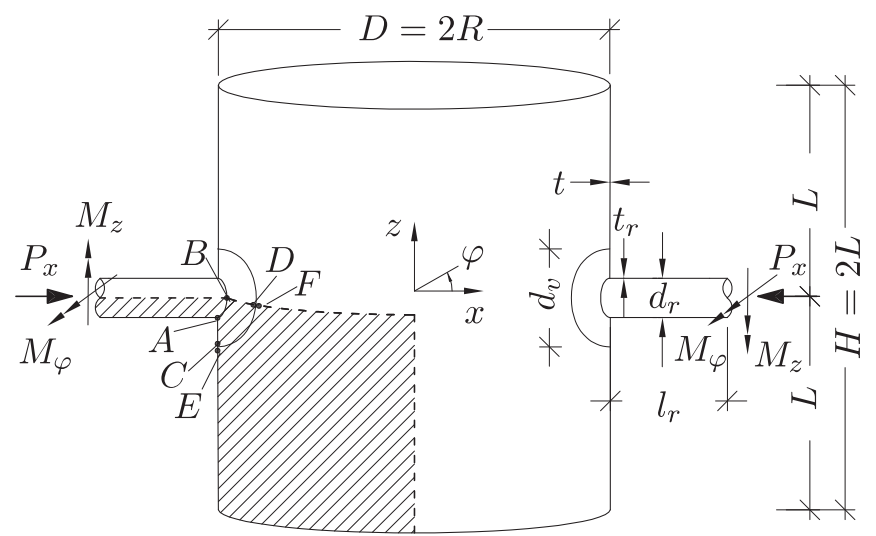

Abbildung 7.1: Kreiszylinderschale mit kreisrunden, scheibenförmigen Verstärkungsblechen im Bereich des Stutzenanschlusses

perfekten Strukturen liegt das aufgesetzte Bleche eben auf dem Mantelblech. Dabei können im verformten Zustand Druck- und Reibkräfte zwischen beiden Blechen übertragen werden.

Für die numerischen Untersuchungen an unverstärkten Kreiszylinderschalen wird das in den Abschnitten 3.4 und 5.10 beschriebene FE-Modell verwendet. Zusätzlich wird ein Verstärkungsblech, das ebenfalls mit Schalenelementen modelliert wird, auf die Kreiszylinderschale gelegt. Der Kontakt zwischen Verstärkungs- und Mantelblech wird durch ein Kontakt-Ziel-Elementenpaar simuliert, auf dessen Eigenschaften in Abschnitt 3.2 eingegangen wurde. Zur Modellierung der Kreiszylinderschalen und der Verstärkungsbleche werden Schalenelemente verwendet, deren Knoten in der Schalenmittelfläche liegen. Aus diesem Grund muss bei der Kontatkdetektion die Dicke der Schalenelemente berücksichtigt werden. Die Kontaktelemente werden auf die virtuelle Unterseite des Verstärkungsbleches gelegt. Auf die virtuelle Oberseite des Mantelbleches der Kreiszylinderschale werden die Zielelemente gelegt. Bei Kontakt zwischen diesen beiden Oberflächen werden Druckkräfte normal zur Kontaktfläche und Reibkräfte tangential zur Kontaktfläche in Abhängigkeit vom Reibkoeffizient $\mu$ übertragen. Die Schweißnähte an den Rändern der Platte werden berücksichtigt, indem alle translatorischen und rotatorischen Freiheitsgrade der Knoten am Plattenrand mit denen der Zylinderwand gekoppelt werden. Das Prinzip dieses FE-Modells ist in Abbildung 7.2 (b) dargestellt. 
(a)

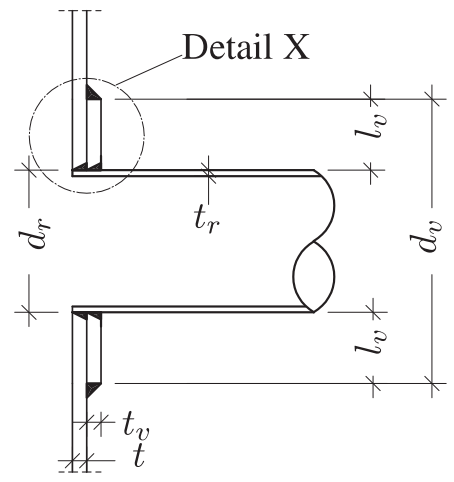

(b) Detail X

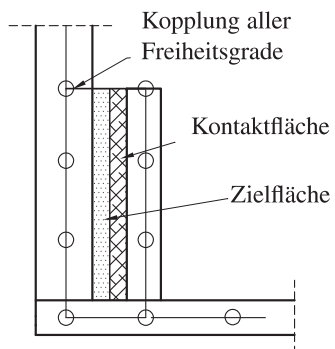

Abbildung 7.2: (a) Schnitt durch den Stutzenanschluss der Kreiszylinderschale mit einem aufgesetzten Verstärkungsblech und (b) FE-Modell von Detail A

\subsubsection{Eingesetzte Verstärkungsbleche}

Eine andere Verstärkungsvariante besteht darin, ein Verstärkungsblech mit der Dicke $t_{v}$ direkt in das Mantelblech einzusetzen. Dabei kann das Verstärkungsblech symmetrisch zur Schalenmittelfäche (Abbildung 7.3 (a)) des Mantelbleches eingesetzt werden. Aus Funktionsgründen wird dieses aber in der Regel exzentrisch (Abbildung 7.4 (a)) eingesetzt.

Da für die Kreiszylinderschalen Schalenelemente verwendet werden, deren Knoten auf der Schalenmittelfläche liegen und Schalenelemente mit exzentrischer Schalenmittelfläche nicht zur Verfügung stehen, wird bei exzentrisch eingesetzten Verstärkungsblechen eine zusätzliche Schicht Schalenelemente modelliert. Die Mittellinie dieser Schalenelemente hat einen Abstand von

$$
e_{v}=\frac{t_{v}}{2}
$$

zur Mittellinie der Kreiszylinderschale. Der Verbund zwischen den Schalenelementen wird durch das in Abschnitt 7.1.2 beschriebene Kontakt-ZielElementenpaar hergestellt, wobei die Kontaktfläche Normal- und Schubkräfte voll übertragen muss. Die angeschrägten Kanten werden im FE-Modell nicht abgebildet. Auf der sicheren Seite liegend werden die abgeschrägten Anteile vernachlässigt und der in den Berechnungen angenommene Durchmesser $d_{v}$ des Verstärkungsbleches durch das Ende der vollen Querschnittssverstärkung begrenzt. 
(a)

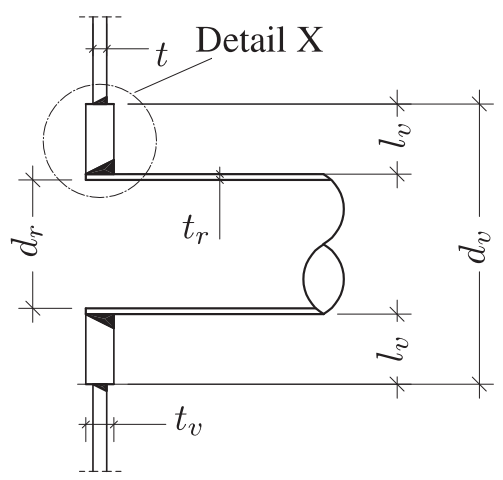

(b) Detail X

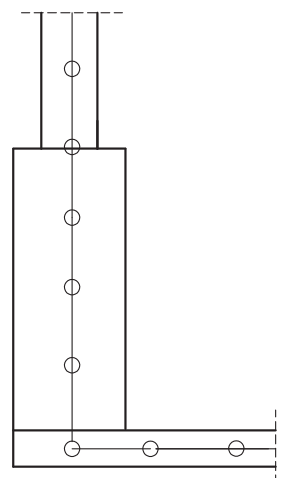

Abbildung 7.3: (a) Schnitt durch den Stutzenanschluss der Kreiszylinderschale mit einem symmetrisch zur Schalenmittelfläche des Mantelbleches eingesetzten Verstärkungsblech und (b) FE-Modell von Detail A

(a)

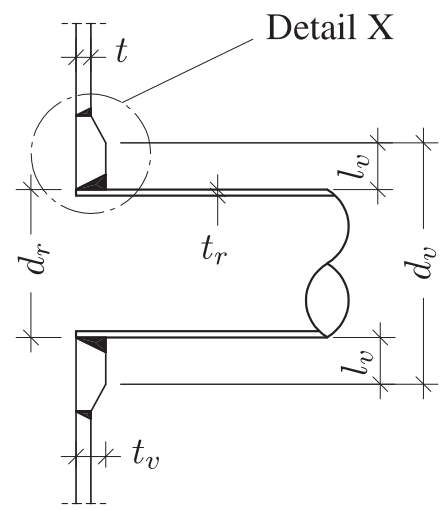

(b) Detail X

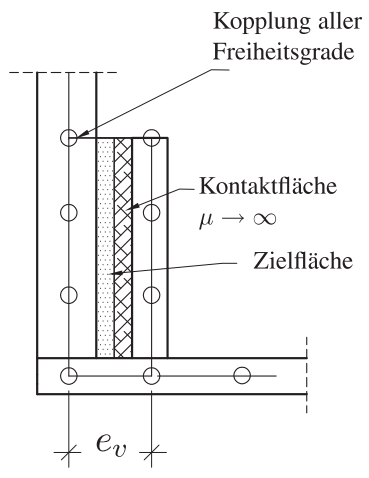

Abbildung 7.4: (a) Schnitt durch den Stutzenanschluss der Kreiszylinderschale mit einem exzentrisch zur Schalenmittelfläche eingesetzten Verstärkungsblech und (b) FE-Modell von Detail A 


\subsubsection{Einfluss unterschiedlicher Ausführungen von Ver- stärkungsblechen auf den Spannungsverlauf}

Bei aufgesetzten Verstärkungsblechen werden in der Kontaktfuge zwischen Mantel- und Verstärkungsblech Druck- und Reibungskräfte übertragen, wenn beide Bleche eben aufeinander liegen. In der Realität ist allerdings oft ein Spalt zwischen den beiden Blechen vorhanden, so dass im ungünstigsten Fall die Kräfte nur über die Schweißverbindung übertragen werden. Die Reibung zwischen zwei Stahlblechen wird mit dem Reibbeiwert $\mu$ erfasst, der im Schrifttum mit Werten zwischen 0,3 und 0,5 angegeben ist. Aufgrund von Verschmutzungen oder Oberflächenbehandlungen kann der Reibbeiwert in der Kontaktfuge weitaus geringer sein, so dass kaum Reibkräfte übertragen werden und im ungünstigsten Fall eine schubweiche Verbindung zwischen Verstärkungsblech und Schalenwand besteht. Auf der sicheren Seite liegend wird in den folgenden Untersuchungen bei aufgesetzten Verstärkungsblechen von einer vollkommen schubweichen Verbindung ausgegangen. Dies wird durch Ansatz einer effektiven Verstärkungsblechdicke $t_{v, \text { eff }}$ berücksichtigt, die bei aufgesetzten Verstärkungsblechen auf die Addition der Trägheitsmomente basiert und sich aus der Dicke $t_{v}$ mit

$$
t_{v, \text { eff }}=t \sqrt[3]{\left(\frac{t_{v}}{t}\right)^{3}+1}
$$

und bei eingesetzten Verstärkungsblechen mit

$$
t_{v, e f f}=t_{v}
$$

ergibt. Der Einfluss der unterschiedlichen Ausführungen von Verstärkungsblechen auf den Spannungsverlauf im Stutzenanschlussbereich wird zunächst an einer Kreiszylinderschale unter einer Einzellast $P_{x}$ untersucht. Dabei wurden folgende Ausführungsvarianten von Verstärkungsblechen betrachtet:

(V1) Kreiszylinderschale mit symmetrisch zur Mittellinie des Mantelbleches eingesetztem Verstärkungsblech,

(V2) Kreiszylinderschale mit exzentrisch zur Mittellinie des Mantelbleches eingesetztem Verstärkungsblech,

(V3) Kreiszylinderschale mit aufgesetztem Verstärkungsblech mit schubweichem Verbund zwischen Verstärkungs- und Mantelblech,

(V4) Kreiszylinderschale mit aufgesetztem Verstärkungsblech mit schubweichem Verbund und einem Spalt von $t / 10$ zwischen Verstärkungsblech und Mantelblech. 
Die Verstärkungsbleche haben bei allen Varianten eine effektive Wanddicke von $t_{v, e f f}=2,0 t$. Die Verläufe der Umfangsspannungen, die sich für die Ausführungsvarianten (V1) bis (V4) ergeben, sind in Abbildung 7.5 entlang des Umfangs der Kreiszylinderschale aufgetragen. Dabei wurden die Umfangsspannungen $\sigma_{\varphi, v e r}^{*}$ der verstärkten Kreiszylinderschale mit der maximalen Spannung $\sigma_{\varphi, \max , P_{x}}^{*}$ am Stutzenanschluss der unverstärkten Kreiszylinderschale normiert. Es sind die Spannungen an der Außennenseite einer Kreiszylinderschale mit $R / t=1000, L^{*}=30, d^{*}=2,5$ und einem Duchmesserverhältnis des Verstärkungsbleches zum Stutzen $d_{v} / d_{r}=2,0$ mit einer effektiven Wanddicke $t_{v, \text { eff }}=2,0 t$ unter einer radialen Einzellast von $P_{x}^{*}=1,0$ dargestellt.

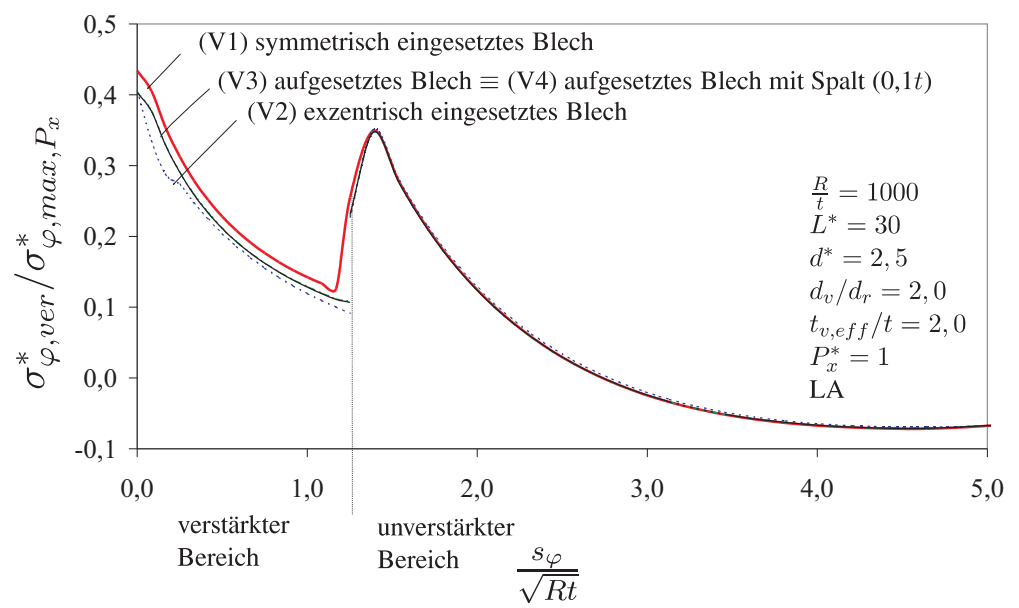

Abbildung 7.5: Verlauf der Spannungen $\sigma_{\varphi, v e r}^{*} / \sigma_{\varphi, \max , P_{x}}^{*}$ für unterschiedliche Verstärkungsvarianten

Der Spannungsverlauf der Variante (V3) (aufgesetztes Blech mit schubweichem Verbund) ist vollkommen identisch mit dem der Variante (V4) (aufgesetztes Blech mit einem Spalt zwischen Verstärkungsblech und Schalenwand). Das bedeutet, dass die Kräfte nur über die Schweißnähte ins Mantelblech eingeleitet werden. Die maximalen Spannungen am Stutzenanschluss sind bei Variante (V1) um rund $7 \%$ größer als die Spannungen anderer Verstärkungsvarianten. Am Übergang zum unverstärkten Manteblech steigen die Spannungen erneut an. Dabei sind keine wesentlichen Einflüsse der unterschiedlichen Verstärkungsvarianten auf die Spannungen im unverstärkten Mantelblech zu beobachten. Es zeigt sich, dass die Auswirkungen der unterschiedlichen Verstärkungsvarianten auf den Spannung- 
verlauf gering sind. Aus diesem Grund werden in den folgenden Untersuchungen vereinfachend und auf der sicheren Seite liegend nur Kreiszylinderschalen mit symmetrisch eingesetzten Verstärkungsblechen betrachtet. Da in der Nähe des Stutzenschlusses die Lastabtragung hauptsächlich über Biegung erfolgt und das Verhältnis der Membranspannungen zu den Biegespannungen bei maximal 1:10 liegt, werden in den folgenden Untersuchungen nur die Randspannungen an der Außenseite der Kreiszylinderschale betrachtet.

\subsection{Kreiszylinderschalen mit Verstärkungsblechen unter radialen Einzellasten $P_{x}$}

\subsubsection{Geometrisch lineare Berechnungen mit elastischem Werkstoffgesetz ( $P_{x}$ ver LA)}

Wie in Abschnitt 6.4.2 für die unverstärkte Kreiszylinderschale gezeigt wurde, treten die größten Spannungen in Umfangsrichtungen entlang des Umfangs der Kreiszylinderschale auf. Da für die verstärkte Kreiszylinderschale ein grundsätzlich anderes Verhalten ausgeschlossen werden kann, werden in diesem Abschnitt ebenfalls nur Umfangsspannungen in Umfangsrichtung betrachtet. Diese Umfangsspannungen $\sigma_{\varphi, v e r}^{*}$ werden mit dem Maximalwert der Spannungen $\sigma_{\varphi, \max , P_{x}}$ in der entsprechenden unverstärkten Kreiszylinderschale ins Verhältnis gesetzt.

Der Spannungsverlauf der verstärkten Kreiszylinderschale kann sowohl von den Geometrieparametern $R / t, L^{*}$ und $d^{*}$ als auch vom Verhältnis der effektiven Dicke $t_{v, \text { eff }}$ des Verstärkungsbleches zur Mantelblechdicke $t$, sowie vom Verhältnis des Verstärkungsblechdurchmessers $d_{v}$ zum Stutzendurchmesser $d_{r}$ beeinflusst werden. Anstelle des Verstärkungsblechdurchmessers $d_{v}$ wird zunächst der Abstand $l_{v}$ zwischen der Außenkante des Stutzens und dem Verstärkungsblechrand nach Abbildung 7.3 betrachtet. In dimensionsloser Form ist

$$
l_{v}^{*}=\frac{l_{v}}{\sqrt{R t_{v, e f f}}}
$$

Da sich bei der unverstärkten Kreiszylinderschale gezeigt hat, dass das $R / t$ Verhältnis und der bezogene Randabstand $L^{*}$ den Spannungsverlauf nur unwesentlich beeinflussen, wird bei den verstärkten Kreiszylinderschalen auf die Variation dieser beiden Größen verzichtet, wobei stets von den ungünstigsten Werten 
$\left(R / t=5000\right.$ und $\left.L^{*}=80\right)$ ausgegangen wird. Die Einflüsse von $d^{*}, t_{v, \text { eff }} / t$ und $l_{v}^{*}$ werden jeweils durch Variation einer Größe nach dem in Tabelle 7.1 angegebenen Schema untersucht.

\begin{tabular}{|c|c|c|c|c|c|}
\hline & $R / t$ & $L^{*}$ & $d^{*}$ & $t_{v, \text { eff }} / t$ & $l_{v}^{*}$ \\
\hline \hline 1 & 5000 & 80 & 3,5 & 2,0 & $0-20$ \\
\hline 2 & 5000 & 80 & $0,5-3,5$ & 2,0 & 4,89 \\
\hline 3 & 5000 & 80 & 3,5 & $1,0-2,0$ & 4,89 \\
\hline
\end{tabular}

Tabelle 7.1: Variationsparameter $\left(P_{x}\right.$ ver LA)

Durch Variation der bezogenen Länge $l_{v}^{*}$ bei konstantem $R / t=5000, L^{*}=80$, $d^{*}=3,5$ und $t_{v, \text { eff }} / t=2,0$ ergeben sich die Spannungsverläufe nach Abbildung 7.6.

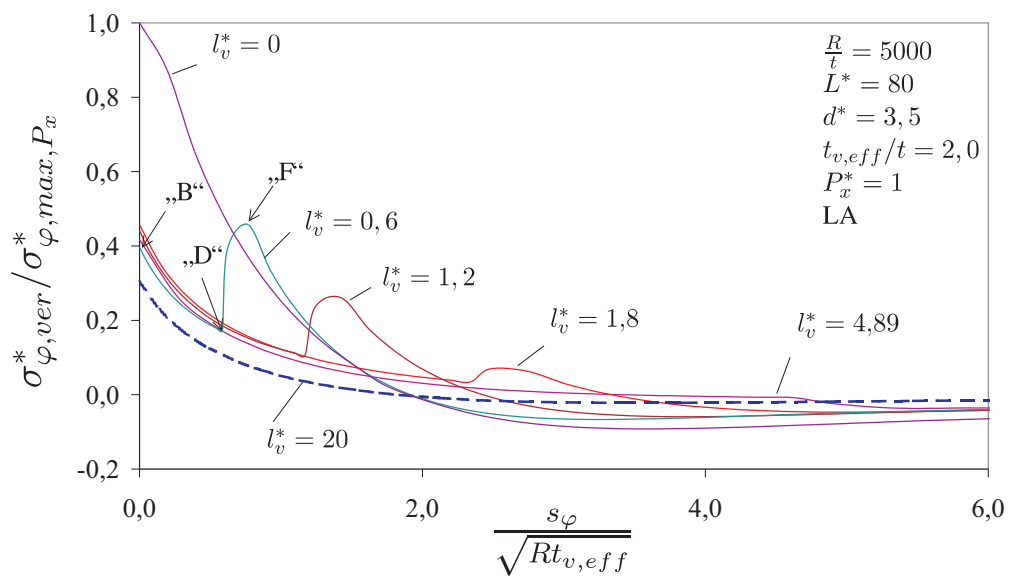

Abbildung 7.6: Verlauf von $\sigma_{\varphi, v e r}^{*} / \sigma_{\varphi, \max , P_{x}}^{*}$ entlang des Umfangs einer Kreiszylinderschale mit $R / t=5000, L^{*}=80, d^{*}=3,5, t_{v, \text { eff }} / t=2,0$ mit unterschiedlichen $l_{v}^{*}$

Am Stutzenanschluss (Stelle B) treten sehr hohe Spannungen auf, die entlang des Verstärkungsbleches schnell abklingen und am Rand des Verstärkungsbleches (Stelle D) sehr viel kleiner sind, als am Stutzenanschluss. Aufgrund der geringeren Blechdicke des unverstärkten Mantelbleches steigen die Spannungen dort erneut an (Stelle F). Mit zunehmendem $l_{v}^{*}$ werden die Kreiszylinderschalen steifer, was zur Folge hat, dass die Spannungen am Stutzenanschluss sinken. 
Gleichzeitig gehen auch die Spannungen, die am Übergang zum unverstärkten Bereich auftreten zurück. Da die Biegespannungen in diesem Bereich um einen Faktor 10 größer sind als die Membranspannungen, entspricht der erneute Spannungsanstieg ungefähr dem Verhältnis der Widerstandsmomente $\left(t_{v, \text { eff }} / t\right)^{2}$ des Verstärkungsblechs zum Mantelblech.

Ziel einer sinnvollen Spannungsreduktion ist es, die Abmessungen der Verstärkungsbleche so zu wählen, dass die Spannungen am äußeren Rand (Stelle D) des Verstärkungsbleches soweit abgeklungen sind, dass die Spannungen im unverstärkten Mantelblech (Stelle F) kleiner sind als die Spannungen am Stutzenanschluss. Um diese Forderung zu erfüllen, müssen die Verstärkungsbleche mit einer Mindestlänge $l_{v, \text { min }}^{*}$ ausgeführt werden. Auf der sicheren Seite liegend ist $l_{v, \text { min }}^{*}$ erreicht, wenn die Spannungen am äußeren Rand des Verstärkungsbleches um den Faktor $\left(t_{v, \text { eff }} / t\right)^{2}$ gegenüber den Spannungen am Stutzenanschluss reduziert wurden.

Wie Abbildung 7.6 zudem zeigt, ist bei einer bezogenen Verstärkungsblechlänge $l_{v}^{*}$ von 4,89 der erneute Anstieg der Spannungen im unverstärkten Mantelblech sehr gering. Dies geht einher mit der Abklinglänge der Biegestörung einer rotationssymmetrisch belasteten Kreiszylinderschale aus Stahl (vgl. Abschnitt $5.5)$.

Im nächsten Schritt wurde der Einfluss des bezogenen Stutzendurchmessers $d^{*}$ auf den Spannungsverlauf untersucht. Dabei hatte das Verstärkungsblech der Kreiszylinderschale ein konstantes $t_{v, \text { eff }} / t$-Verhältnis von 2,0 und eine bezogene Länge von $l_{v}^{*}=4,89$.

Wie anhand der Spannungsverläufe in Abbildung 7.7 deutlich wird, beeinflusst der bezogene Stutzendurchmesser $d^{*}$ den Spannungsverlauf der Kreiszylinderschale. Da der Spannungsunterschied für unterschiedliche $d^{*}$ klein sind, ist es für eine überschlägige Dimensionierung der Verstärkungsbleche jedoch ausreichend, den ungünstigsten Fall, eine Kreiszylinderschale mit $L^{*}=80, R / t=5000$ und $d^{*}=3,5 \mathrm{zu}$ betrachten. Ausgehend von dieser Kreiszylinderschale wurden im nächsten Schritt die Auswirkungen unterschiedlicher Blechdickenverhältnisse $t_{v, e f f} / t$ auf den Spannungsverlauf untersucht. Praktikable und wirtschaftliche Verstärkungsblechdicken ergeben Blechdickenverhältnisse zwischen 1, 2 und 2, 0. Die Spannungsverläufe, die sich durch Variation der Verstärkungsblechdicken ergeben, sind in Abbildung 7.8 entlang des Umfangs der Kreizsylinderschalen aufgetragen.

Dabei wird deutlich, dass die Reduktion der Spannungen umso größer ist, je größer das $t_{v, \text { eff }} / t$-Verhältnis ist. Die Spannungen am Rand des Verstärkungsbleches („D“) können durch Abminderung der Spannungen am Stutzenan- 


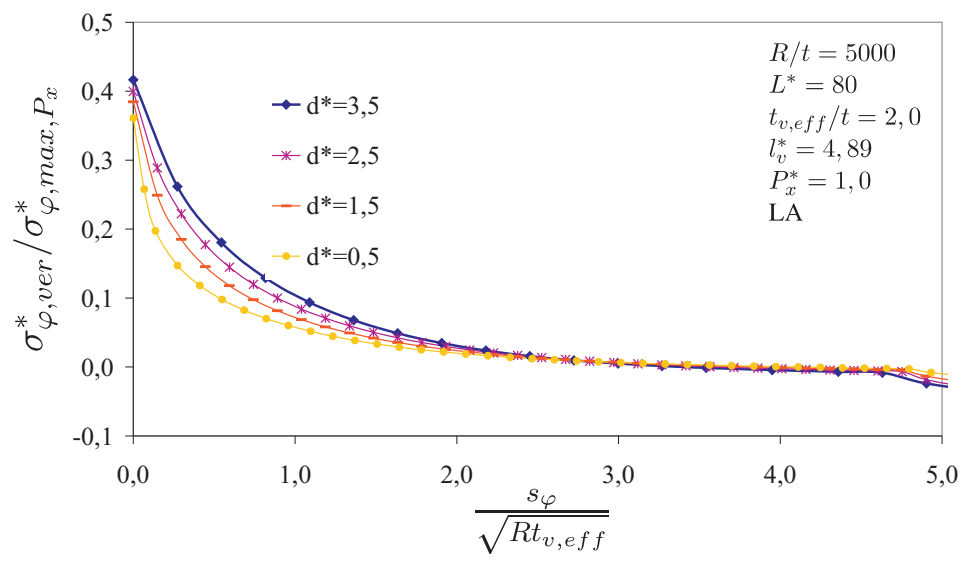

Abbildung 7.7: Spannungsverlauf $\sigma_{\varphi, v e r}^{*} / \sigma_{\varphi, \max , P_{x}}^{*}$ entlang des Umfangs einer Kreiszylinderschale mit $R / t=5000, L^{*}=80, t_{v, \text { eff }} / t=2,0, l_{v}^{*}=4,89$ und $0,5 \leq d^{*} \leq 3,5$

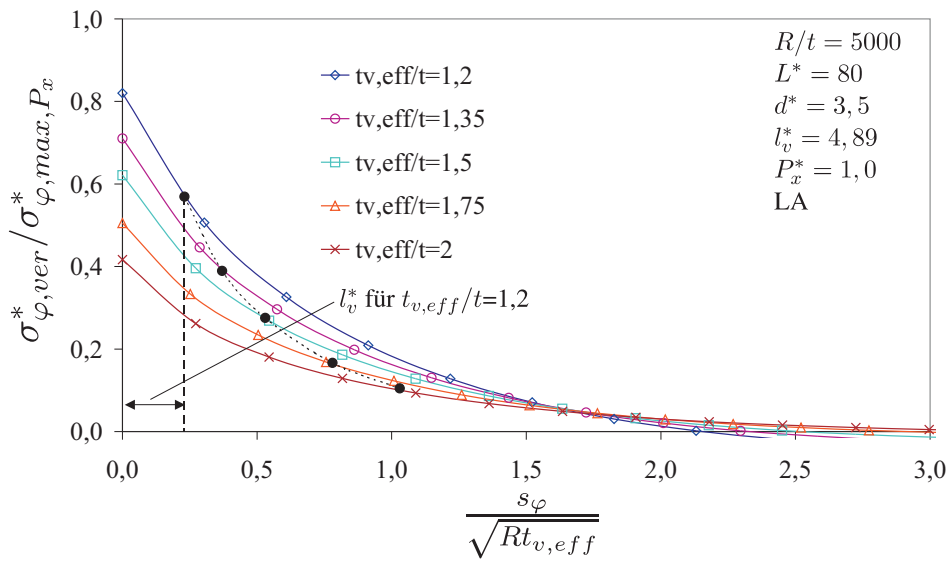

Abbildung 7.8: Spannungsverlauf $\sigma_{\varphi, v e r}^{*} / \sigma_{\varphi, \max , P_{x}}^{*}$ entlang des Umfangs einer Kreiszylinderschale mit $R / t=5000, L^{*}=80, d^{*}=3,4, l_{v}^{*}=4,89$ und $1,2 \leq t_{v, \text { eff }} / t \leq 2,0$ 
schluss (,B“) mit $\left(t / t_{v, e f f}\right)^{2}$ ermittelt werden. Diese Spannungsverhältnisse $\sigma_{\varphi, v e r}^{*} / \sigma_{\varphi, \text { max }, P_{x}}^{*} \cdot\left(t / t_{v, \text { eff }}\right)^{2}$ sind in Abbildung 7.8 durch Punkte markiert. Die entsprechenden Stützstellen auf der horizontalen Achse des Diagramms sind dabei die minimalen Blechlängen $l_{v, \text { min }}^{*}$, die in Abhängigkeit von $t_{v, \text { eff }} / t$ mit

$$
l_{v, \min }^{*}=\left(\frac{t_{v, e f f}}{t}\right)-1
$$

abgeschätzt werden können. Das Verhältniss der Spannungen $\sigma_{\varphi, v e r}^{*} / \sigma_{\varphi, \max , P_{x}}^{*}$ am Stutzenanschluss und am Übergang zum unverstärkten Mantelblech kann überschlägig mit

$$
\frac{\sigma_{\varphi, v e r}^{*}}{\sigma_{\varphi, \max , P_{x}}^{*}}=1,16\left(\frac{t}{t_{v, \text { eff }}}\right)-0,16
$$

bestimmt werden.

Der Zusammenhang zwischen dem Verhältnis des Durchmessers $d_{v}$ des Verstärkungsbleches zum Durchmesser $d_{r}$ des Stutzens und der Länge $l_{v}$ des Verstärkungsbleches ist mit

$$
\frac{d_{v}}{d_{r}}=\frac{2 l_{v}+d_{r}}{d_{r}}=\frac{2 l_{v}^{*}}{d^{*}} \sqrt{\frac{t_{v, e f f}}{t}}+1
$$

gegeben.

Aus konstruktiven Gesichtspunkten liegen die Durchmesserverhältnisse $d_{v} / d_{r}$ in der Regel bei maximal 2,0. Das minimale Durchmesserverhältnisse $\left(d_{v} / d_{r}\right)_{\min }$ ist mit $l_{v, \text { min }}^{*}$ nach Gleichung 7.5 und dem entsprechenden $d^{*}$ vorgegeben. $\left(d_{v} / d_{r}\right)_{\min }$ ist in Abhängigkeit vom bezogenen Stutzendurchmesser $d^{*}$ und den Blechdickenverhältnissen $t_{v, \text { eff }} / t$ in Abbildung 7.9 aufgetragen. Die maximalen Spannungsverhältnisse $\sigma_{\varphi, v e r}^{*} / \sigma_{\varphi, \text { max }, P_{x}}^{*}$, die im Stutzenanschlussbereich auftreten, sind in Abbildung 7.10 über $t_{v, \text { eff }} / t$ aufgetragen. 


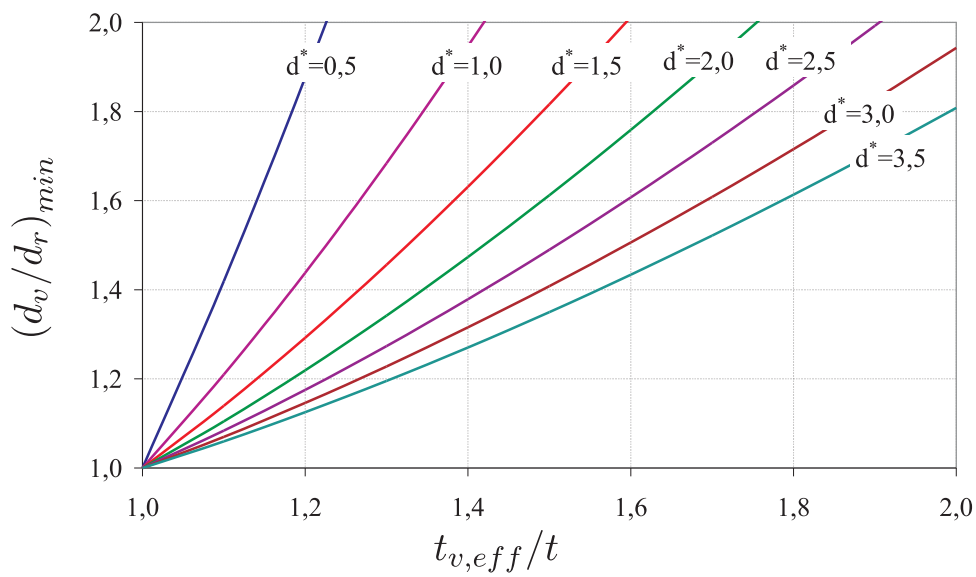

Abbildung 7.9: Mindestdurchmesserverhältnisse $\left(d_{v} / d_{r}\right)_{\text {min }}$ in Abhängigkeit von $d^{*}$ und $t_{v, e f f} / t$

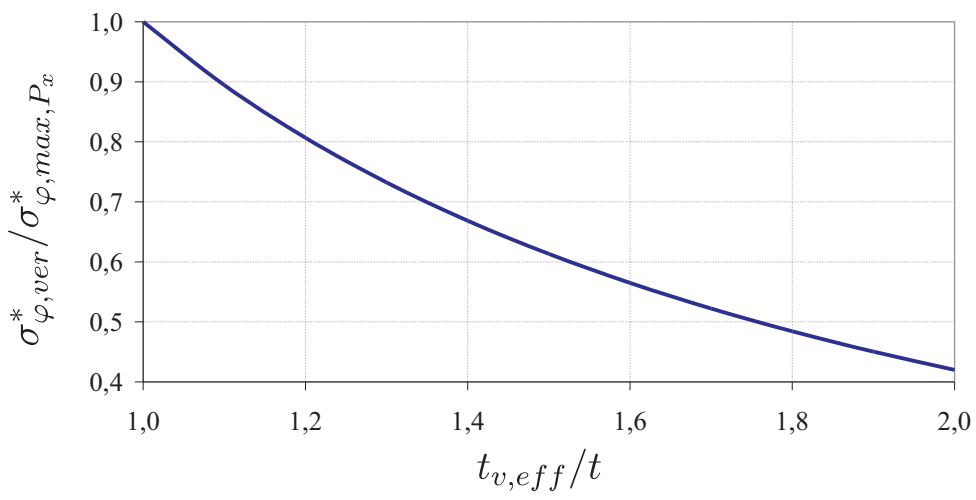

Abbildung 7.10: Spannungsverhältnis $\sigma_{\varphi, v e r}^{*} / \sigma_{\varphi, \max , P_{x}}^{*}$ in Anhängigkeit von $t_{v, e f f} / t$ 


\subsubsection{Geometrisch nichtlineare Berechnungen mit elastischem Werkstoffgesetz ( $P_{x}$ ver GNA)}

In diesem Abschnitt werden Kreiszylinderschalen mit Durchmesserverhältnissen $d_{v} / d_{r}$ zwischen 1,0 und 2,0 und Blechdickenverhältnisse $t_{v, \text { eff }} / t$ bis 2,0 untersucht. Die Parameter $R / t, L^{*}$ und $d^{*}$ wirken sich in gleicher Weise, wie für die unverstärkten Kreiszylinderschale in Abschnitt 6.4.3 gezeigt wurde, auch auf das Tragverhalten der verstärkten Kreiszylinderschale aus. Der Einfluss der Parameter $d_{v} / d_{r}$ und $t_{v, \text { eff }} / t$ wurden nach dem in Tabelle 7.2 aufgeführten Variationsschema untersucht.

\begin{tabular}{|c|c|c|c|c|}
\hline$R / t$ & $L^{*}$ & $d^{*}$ & $d_{v} / d_{r}$ & $t_{v, \text { eff }} / t$ \\
\hline \hline 400 & 20 & 1,5 & 2,0 & $1,0-2,0$ \\
\hline 400 & 20 & 1,5 & $1,0-2,0$ & 2,0 \\
\hline
\end{tabular}

Tabelle 7.2: Variationsparameter $\left(P_{x}\right.$ ver GNA)

In Abbildung 7.11 sind die Last-Verformungskurven einer Kreiszylinderschale mit $L^{*}=20, R / t=400$ und $d^{*}=1,5$ mit einem Verstärkungsblech mit $d_{v} / d_{r}=2,0$ und $t_{v, \text { eff }} / t$ zwischen 1,0 und 2,0 aufgetragen. Je größer $t_{v, e f f} / t$ ist, umso deutlicher ist das lokale Maximum in den Last-Verformungskurven ausgeprägt. Durch die Verstärkungsbleche im Stutzenanschlussbereich nimmt die Steifigkeit der Kreiszylinderschale zu. Das hat zur Folge, dass die Lastabtragung über Biegung gegenüber der Membrantragwirkung abnimmt. Dieses Verhältnis wirkt sich auf das Tragverhalten der Kreiszylinderschale aus, das in Abschnitt 4.3 ausführlich erörtert wurde. Je größer das Blechdickenverhältnis $t_{v, \text { eff }} / t$ ist, umso weniger verformt sich der verstärkte Bereich der Kreiszylinderschale unter der Last $P_{x}$.

Für den Grenzfall, wenn das Verstärkungsblech unendlich steif ist, entspricht die Last-Verformungskurve der verstärkten Kreiszylinderschale der einer unverstärkten Kreiszylinderschale mit einem bezogenen Stutzendurchmesser

$$
d^{*}=d_{v e r}^{*} \cdot \frac{d_{v}}{d_{r}}=1,5 \cdot 2,0=3,0
$$

In Abbildung 7.12 sind die Last-Verformungskurven einer Kreiszylinderschale dargestellt, deren Verstärkungsblechdurchmesser $d_{v} / d_{r}$ zwischen 1,0 und 2,0 bei einem konstanten Blechdickenverhältnis $t_{v, \text { eff }} / t$ von 2,0 variiert wurden. Dabei zeigt sich, dass die kritischen Lasten von $d_{v} / d_{r}$ abhängig sind und mit steigendem $d_{v} / d_{r}$ ebenfalls ansteigen. Die Steigung $c_{P_{x}, v e r}^{*}$ der Kurven im linearen Bereich ist ebenfalls umso größer, je größer $t_{v, e f f} / t$ und $d_{v} / d_{r}$ ist. 


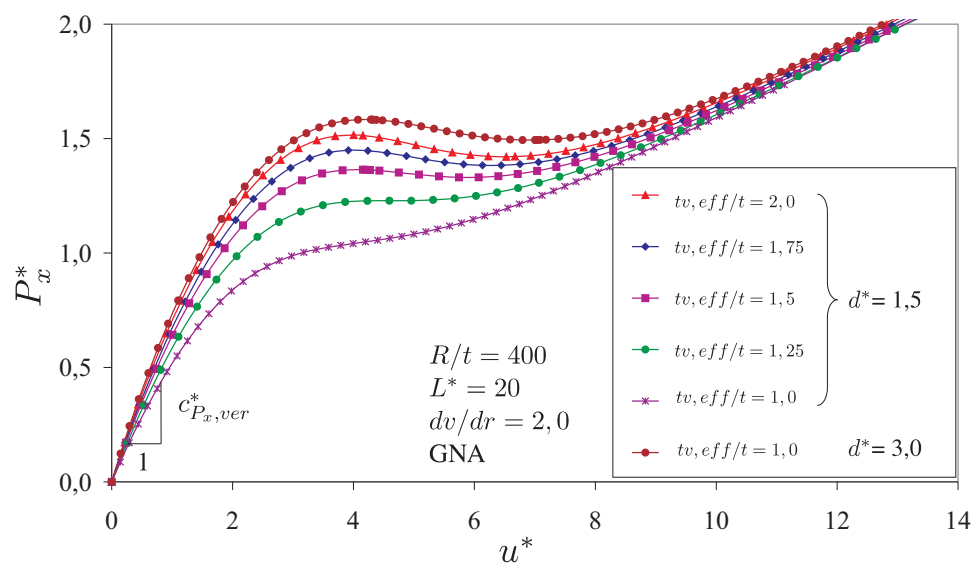

Abbildung 7.11: Last-Verformungskurve einer Kreiszylinderschale mit $L^{*}=20$, $R / t=400, d^{*}=1,5$ und einem Verstärkungsblech mit $d_{v} / d_{r}=2,0$ und $1,0 \leq t_{v, \text { eff }} / t \leq 2,0$, sowie einer unverstärkten Kreiszylinderschale mit $d^{*}=3,0$

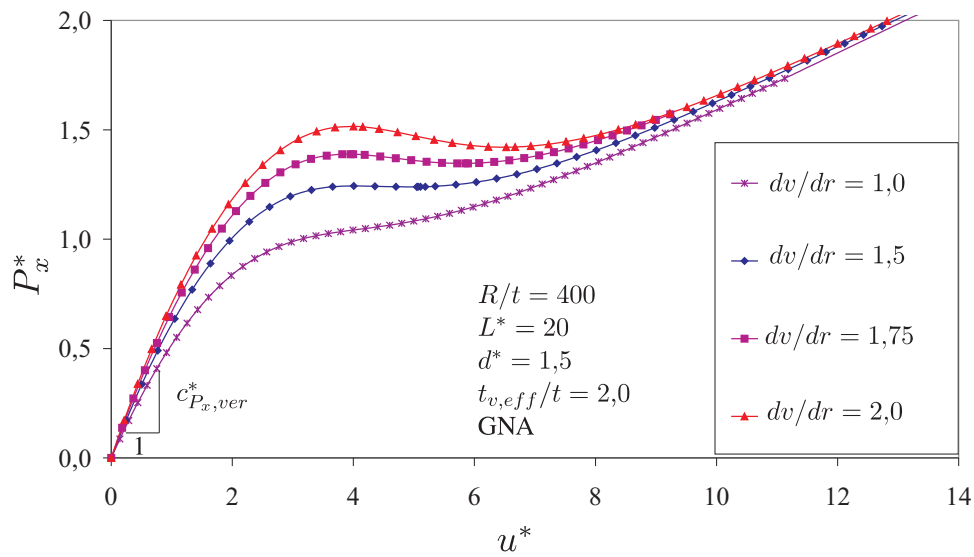

Abbildung 7.12: Last-Verformungskurve einer Kreiszylinderschale mit $L^{*}=20$, $R / t=400, d^{*}=1,5$ mit einem Verstärkungsblech mit $t_{v, \text { eff }} / t=2,0$ und $1,0 \leq d_{v} / d_{r} \leq 2,0$ 
In umfangreichen Serienberechnungen wurden die Parameter $L^{*}, R / t, d^{*}$ sowie $d_{v} / d_{r}$ und $t_{v, \text { eff }} / t$ variiert und die kritischen Lasten $P_{c r i t, G N A, v e r}^{*}$ und die Anfangssteifigkeiten $c_{P_{x}, v e r}^{*}$ ermittelt. Die kritischen Lasten $P_{c r i t, G N A, v e r}^{*}$ und die Anfangssteifigkeiten $c_{P_{x}}^{*}$,ver der verstärkten Kreiszylinderschale steigen, wie in Abbildung 7.13 und Abbildung 7.14 für Verstärkungsbleche mit $d_{v} / d_{r}=2,0$ und $t_{v, \text { eff }} / t=2,0$ gezeigt wird, mit steigendem $d^{*}$ und abnehmendem $L^{*}$ an.

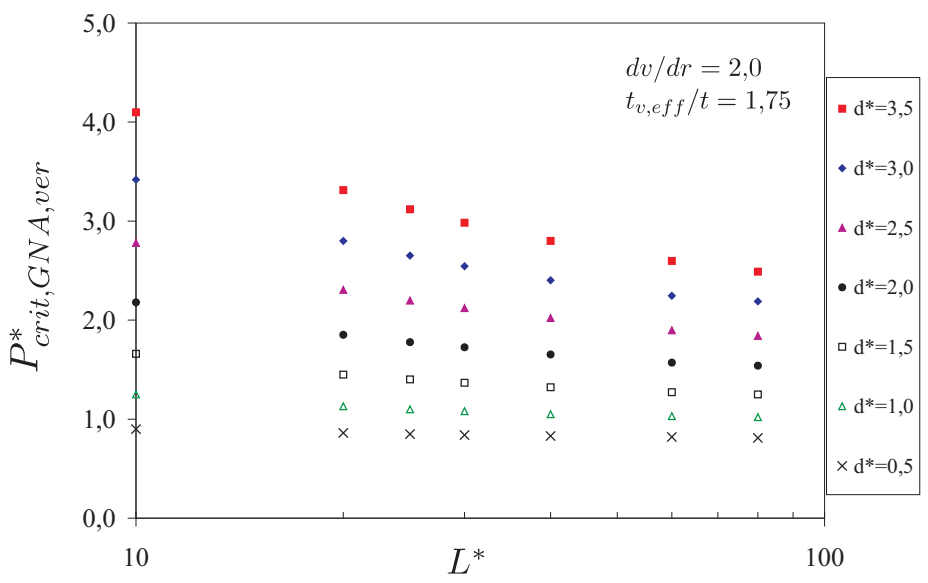

Abbildung 7.13: Kritische Lasten $P_{x, \text { crit }, G N A \text {,ver }}$ von Kreiszylinderschalen mit $10 \leq L^{*} \leq 80,0,5 \leq d^{*} \leq 3,5$ und Verstärkungsblechen mit $t_{v, \text { eff }} / t=1,75$ und $d_{v} / d_{r}=2,0$

Die kritischen Lasten $P_{x, \text { crit, GNA,ver }}^{*}$ in Abhängigkeit von den untersuchten Parametern $L^{*}, R / t, d^{*}, d_{v} / d_{r}$ und $t_{v, \text { eff }} / t$ können mit

$$
P_{x, \text { crit }, \text { GNA }, \text { ver }}^{*}=P_{0}^{*}+\frac{\psi}{L^{*}}
$$

bestimmt werden. $P_{0}^{*}$ ist in Abbildung 7.15 und $\psi$ in Abbildung 7.16 für Verstärkungsbleche mit $d_{v} / d_{r}=1,5$ aufgetragen. Für Verstärkungsbleche mit $d_{v} / d_{r}=2,0$ sind diese Parameter in Abbildung 7.17 und Abbildung 7.18 angegeben. Liegen die Abmessungen der Kreiszylinderschalen zwischen den in Abbildung 7.15 bis 7.18 angegebenen Werten, so kann linear interpoliert werden.

Die Anfangssteifigkeiten $c_{P_{x}, v e r}^{*}$ in Abhängigkeit von den untersuchten Parametern $L^{*}, R / t, d^{*}, d_{v} / d_{r}$ und $t_{v, e f f} / t$ können mit

$$
c_{P_{x}, \text { ver }}^{*}=c_{0, P_{x}}^{*} \cdot\left(L^{*}\right)^{\chi}
$$




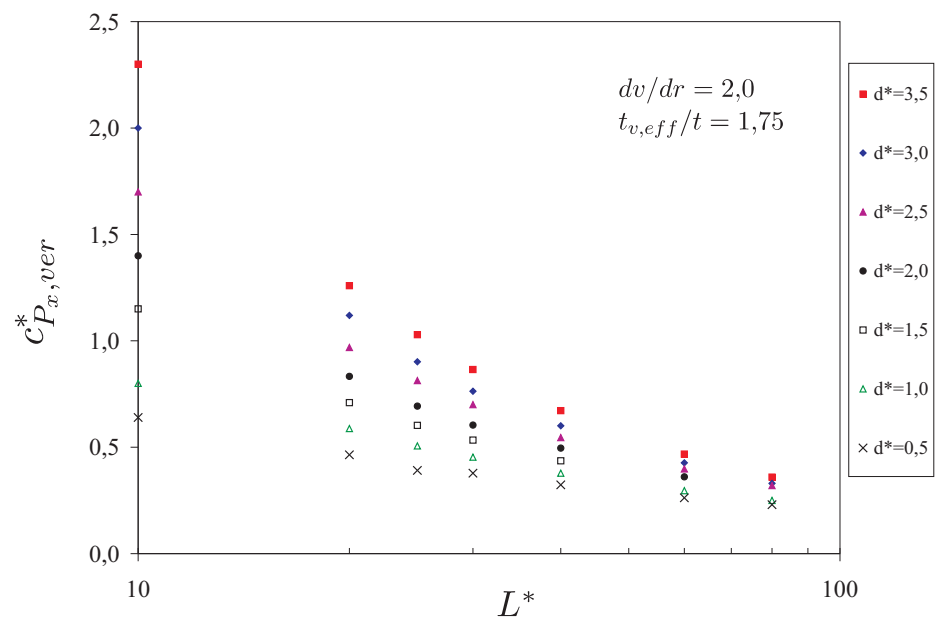

Abbildung 7.14: Anfangssteifigkeiten $c_{P_{x}, v e r}^{*}$ von Kreiszylinderschalen mit $10 \leq L^{*} \leq 80,0,5 \leq d^{*} \leq 3,5$ und Verstärkungsblechen mit $t_{v, \text { eff }} / t=1,75$ und $d_{v} / d_{r}=2,0$

bestimmt werden. $c_{0, P_{x}}^{*}$ ist in Abbildung 7.19 und $\chi$ in Abbildung 7.20 für Verstärkungsbleche mit $d_{v} / d_{r}=1,5$ aufgetragen. Für Verstärkungsbleche mit $d_{v} / d_{r}=2,0$ sind diese Parameter in Abbildung 7.21 und Abbildung 7.22 angegeben. Liegen die Abmessungen der Kreiszylinderschalen zwischen den in Abbildung 7.19 bis 7.22 angegebenen Werten, so kann linear interpoliert werden. 


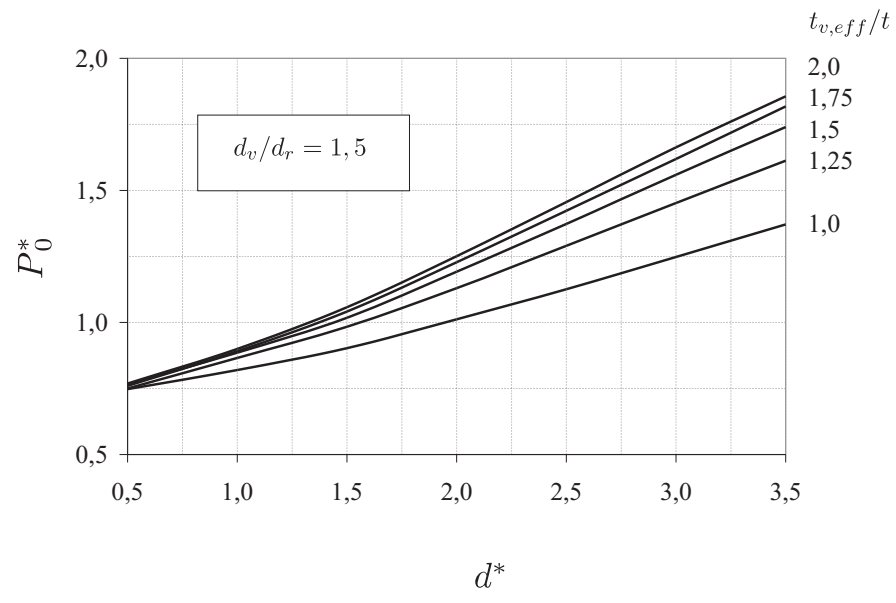

Abbildung 7.15: $P_{0}^{*}$ zur Bestimmung der kritischen Lasten $P_{x, \text { crit }, G N A, v e r}^{*}$ von Kreiszylinderschalen mit $10 \leq L^{*} \leq 80,0,5 \leq d^{*} \leq 3,5$ und Verstärkungsblechen mit $1,0 \leq t_{v, \text { eff }} / t \leq 2,0$ für $d_{v} / d_{r}=1,5$

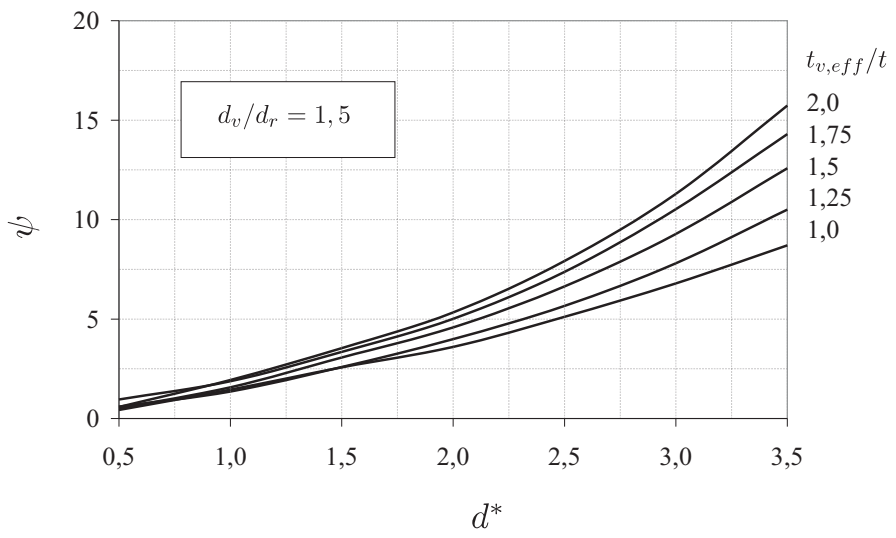

Abbildung 7.16: $\psi$ zur Bestimmung der kritischen Lasten $P_{x, \text { crit, GNA,ver von }}^{*}$ Kreiszylinderschalen mit $10 \leq L^{*} \leq 80,0,5 \leq d^{*} \leq 3,5$ und Verstärkungsblechen mit $1,0 \leq t_{v, \text { eff }} / t \leq 2,0$ für $d_{v} / d_{r}=1,5$ 


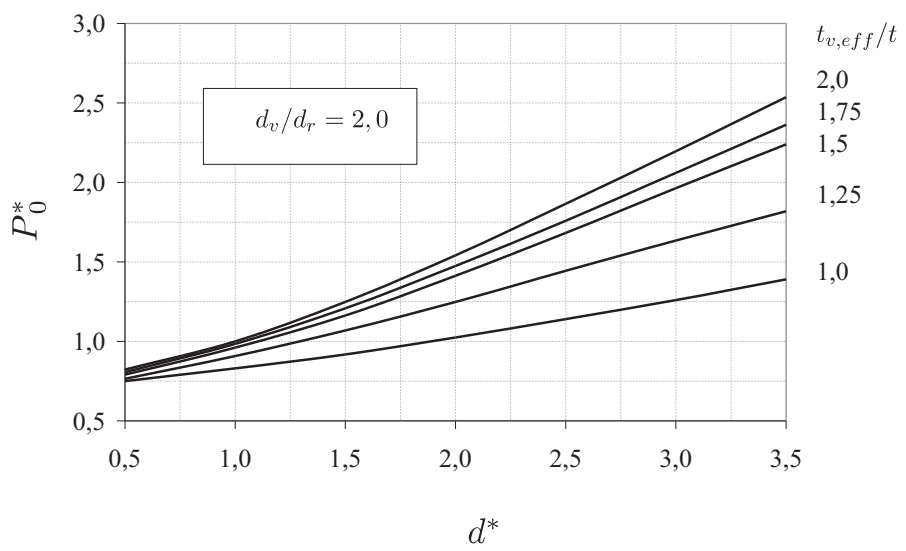

Abbildung 7.17: $P_{0}^{*}$ zur Bestimmung der kritischen Lasten $P_{x, \text { crit }, G N A, v e r}^{*}$ von Kreiszylinderschalen mit $10 \leq L^{*} \leq 80,0,5 \leq d^{*} \leq 3,5$ und Verstärkungsblechen mit $1,0 \leq t_{v, \text { eff }} / t \leq 2,0$ für $d_{v} / d_{r}=2,0$

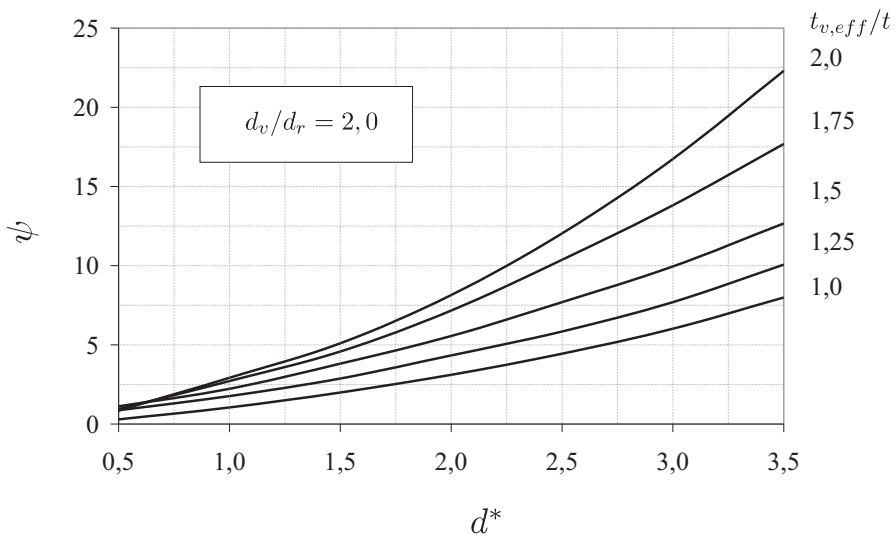

Abbildung 7.18: $\psi$ zur Bestimmung der kritischen Lasten $P_{x, \text { crit }, G N A, v e r}^{*}$ von Kreiszylinderschalen mit $10 \leq L^{*} \leq 80,0,5 \leq d^{*} \leq 3,5$ und Verstärkungsblechen mit $1,0 \leq t_{v, \text { eff }} / t \leq 2,0$ für $d_{v} / d_{r}=2,0$ 


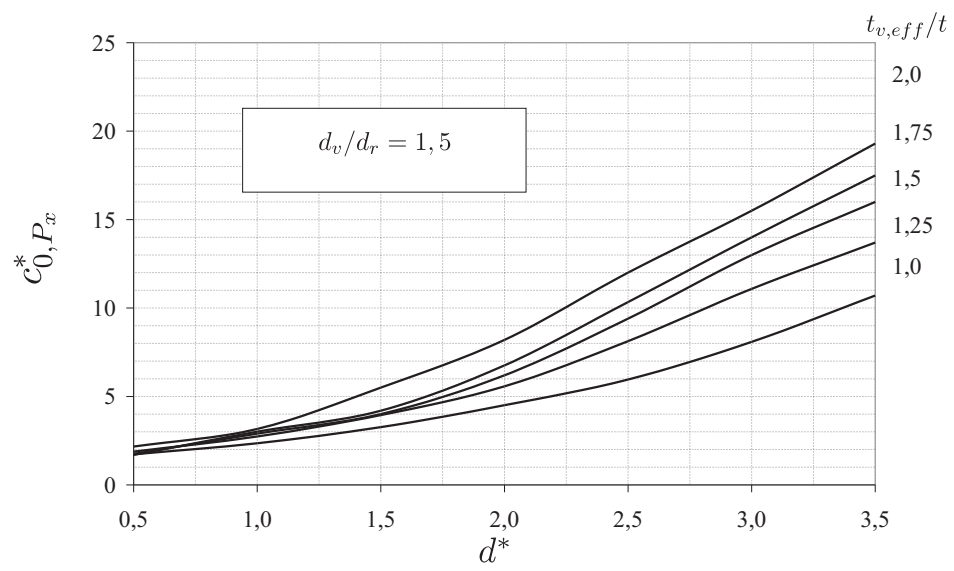

Abbildung 7.19: $c_{0, P_{x}}^{*}$ zur Bestimmung der Anfangssteifigkeit $c_{P_{x}, v e r}^{*}$ von Kreiszylinderschalen mit $10 \leq L^{*} \leq 80,0,5 \leq d^{*} \leq 3,5$ und Verstärkungsblechen mit $1,0 \leq t_{v, \text { eff }} / t \leq 2,0$ für $d_{v} / d_{r}=1,5$

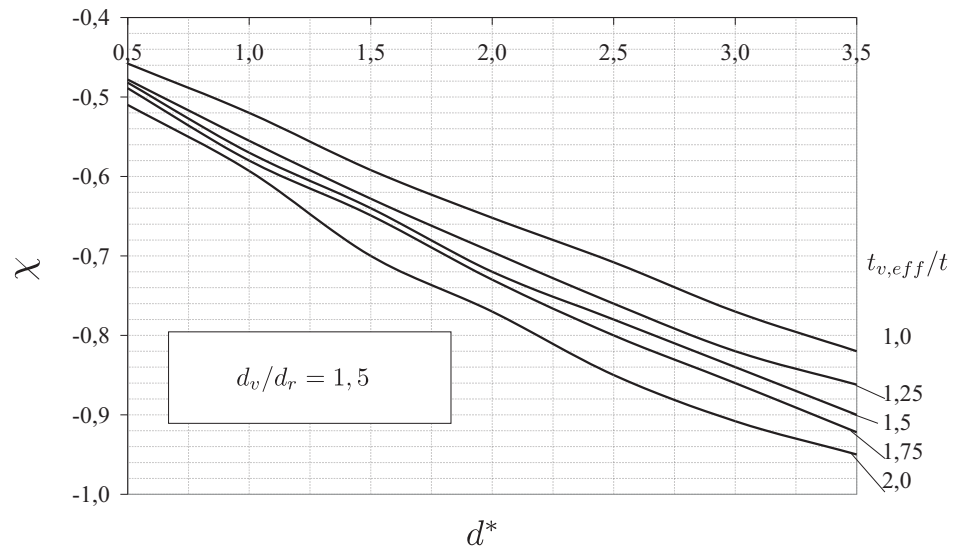

Abbildung 7.20: $\chi$ zur Bestimmung der Anfangssteifigkeit $c_{P_{x}, v e r}^{*}$ von Kreiszylinderschalen mit $10 \leq L^{*} \leq 80,0,5 \leq d^{*} \leq 3,5$ und Verstärkungsblechen mit $1,0 \leq t_{v, \text { eff }} / t \leq 2,0$ für $d_{v} / d_{r}=1,5$ 


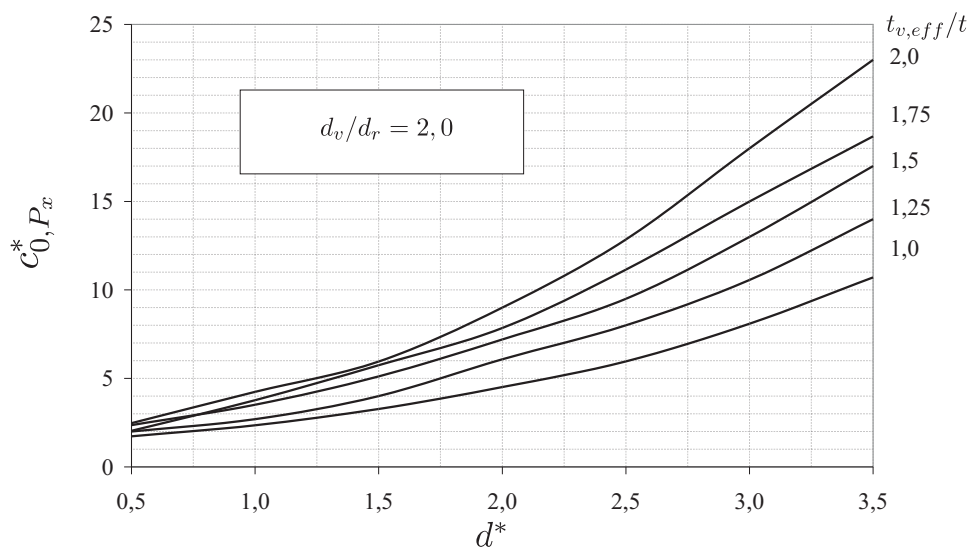

Abbildung 7.21: $c_{0, P_{x}}^{*}$ zur Bestimmung der Anfangssteifigkeit $c_{P_{x}, v e r}^{*}$ von Kreiszylinderschalen mit $10 \leq L^{*} \leq 80,0,5 \leq d^{*} \leq 3,5$ und Verstärkungsblechen mit $1,0 \leq t_{v, \text { eff }} / t \leq 2,0$ für $d_{v} / d_{r}=2,0$

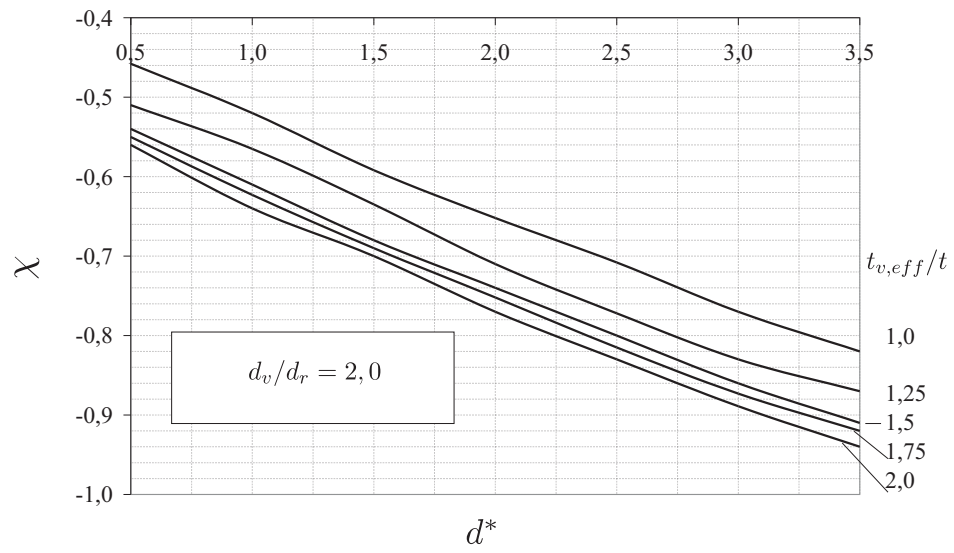

Abbildung 7.22: $\chi$ zur Bestimmung der Anfangssteifigkeit $c_{P_{x}, v e r}^{*}$ von Kreiszylinderschalen mit $10 \leq L^{*} \leq 80,0,5 \leq d^{*} \leq 3,5$ und Verstärkungsblechen mit $1,0 \leq t_{v, \text { eff }} / t \leq 2,0$ für $d_{v} / d_{r}=2,0$ 


\subsubsection{Einfluss materieller Nichtlinearität auf das Tragverhal- ten verstärkter Kreiszylinderschalen}

Bei Berücksichtigung des plastischen Werkstoffverhaltens in den numerischen Berechnungen ist ein Einfluss der Streckgrenze des Werkstoffes zu erwarten. Wie sich bei den Untersuchungen zur unverstärkten Kreiszylinderschale in Abschnitt 6.4.5 gezeigt hatte, kann der Zusammenhang zwischen Streckgrenze und $R / t$ Verhältnis mit dem Faktor $\eta$ erfasst werden. Der Einfluss der Parameter wurde nach dem in Tabelle 7.3 dargestellten Schema untersucht.

\begin{tabular}{|c|c|c|c|c|c|}
\hline$R / t$ & $L^{*}$ & $d^{*}$ & $f_{y}$ & $d_{v} / d_{r}$ & $t_{v, \text { eff }} / t$ \\
\hline \hline $400-800$ & 20 & 1,5 & 360 & 2,0 & 2,0 \\
\hline 400 & 20 & 1,5 & $240-360$ & 2,0 & 2,0 \\
\hline 400 & 20 & 1,5 & 240 & 2,0 & $1,25-2,0$ \\
\hline 400 & 20 & $1,5-3,0$ & 240 & $1,0-2,0$ & 2,0 \\
\hline
\end{tabular}

Tabelle 7.3: Variationsparameter $\left(P_{x}\right.$ ver GMNA)

In den Schritten 1 und 2 wurde das $R / t$-Verhältnis und die Streckgrenze $f_{y}$, und damit der Faktor $\eta$ variiert. Im dritten Schritt wurde das Blechdickenverhältnis variiert. Die Ergebnisse dieser Untersuchungsschritte sind in Abbildung 7.23 dargestellt.

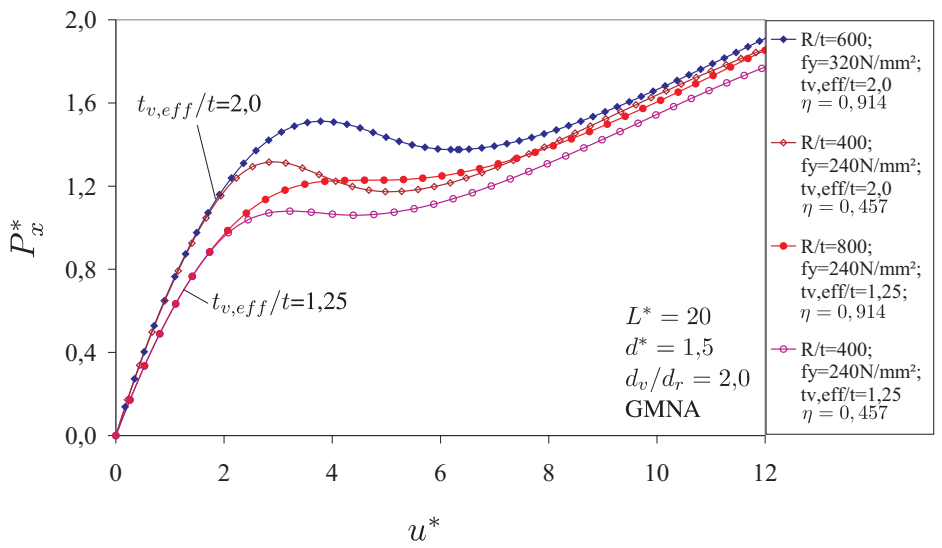

Abbildung 7.23: Last-Verformungskurven von verstärkten Kreiszylinderschalen mit $\left.L^{*}=20, d^{*}=1,5, d_{v} / d_{r}=2,0\right)$ und unterschiedlichen $R / t, f_{y}$ und $t_{v, \text { eff }} / t$ 
Es zeigt sich, dass die kritische Last $P_{x, c r i t, G M N A, v e r}^{*}$ vom Blechdickenverhältnis $t_{v, \text { eff }} / t$ und von $\eta$ beeinflusst wird. Je kleiner $\eta$ oder $t_{v, \text { eff }} / t$ ist, desto geringer ist auch die kritische Last $P_{x, c r i t, G M N A, v e r}^{*}$.

In Abbildung 7.24 sind die Last-Verformungskurve von Kreiszylinderschalen mit $L^{*}=20, d^{*}=1,5$ und $t_{v, \text { eff }} / t=2,0$ bei unterschiedlichen $R / t$-Verhältnissen, Streckgrenzen und Durchmesserverhältnissen dargestellt. Es zeigt sich, dass die kritischen Lasten von $d_{v} / d_{r}$ und von $\eta$ beeinflusst werden. Je kleiner $d_{v} / d_{r}$ oder $\eta$ sind, desto geringer sind die kritischen Lasten.

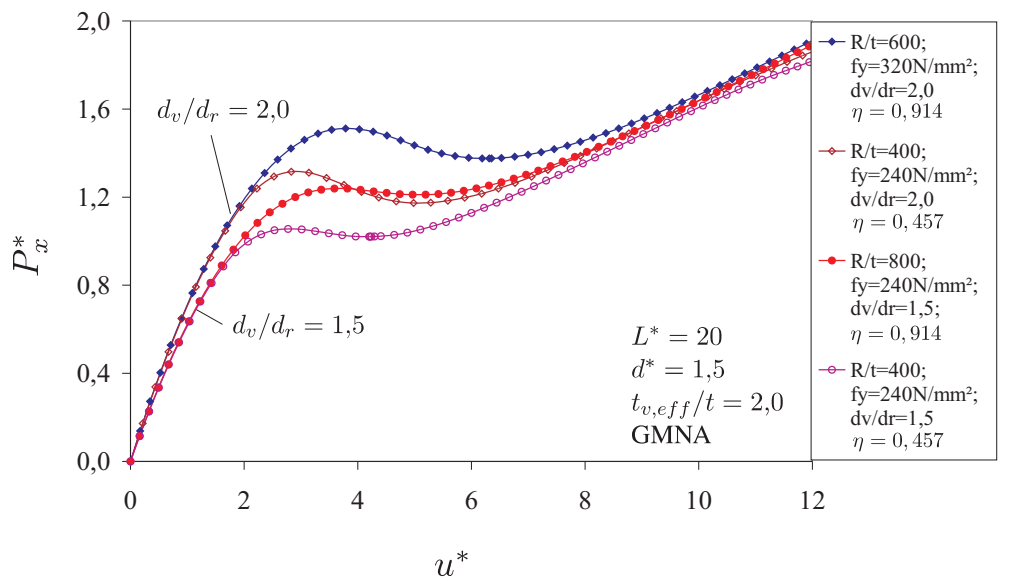

Abbildung 7.24: Last-Verformungskurven von verstärkten Kreiszylinderschalen mit $L^{*}=20, d^{*}=1,5, t_{v, \text { eff }} / t=2,0$ und variablen $R / t, f_{y}$ und $d_{v} / d_{r}$

Die kritischen Lasten $P_{x, c r i t, G M N A, v e r}^{*}$ der verstärkten Kreiszylinderschalen hängen auch vom bezogenen Randabstand $L^{*}$ und dem bezogenen Stutzendurchmesser $d^{*}$ ab. Da sich das Tragverhalten verstärkter Kreiszylinderschalen in Abhängigkeit von diesen Parametern gegenüber unverstärkten Kreiszylinderschalen nicht grundsätzlich ändert, werden diese Last-Verformungskurven nicht gesondert dargestellt.

In Abbildung 7.25 wurde das Verhältnis der kritischen Lasten $P_{x, \text { crit }, G M N A, v e r}^{*}$ zu $P_{x, \text { crit,GNA,ver }}^{*}$ der verstärkten Kreiszylinderschalen über $\eta$ aufgetragen. Es zeigt sich, dass sich alle untersuchten Parameter auf das Tragverhalten auswirken. Es wird zudem deutlich, dass im untersuchten Bereich die Abminderung infolge plastischen Werkstoffverhaltens bei maximal $80 \%$ liegt. Vereinfachend und auf der sicheren Seite liegend können die plastischen Abminderungskurven der un- 
verstärkten Kreiszylinderschalen aus Abbildung 7.25 und die Abminderung nach Gleichung 6.9 auch für die verstärkten Kreiszylinderschalen angewandt werden.

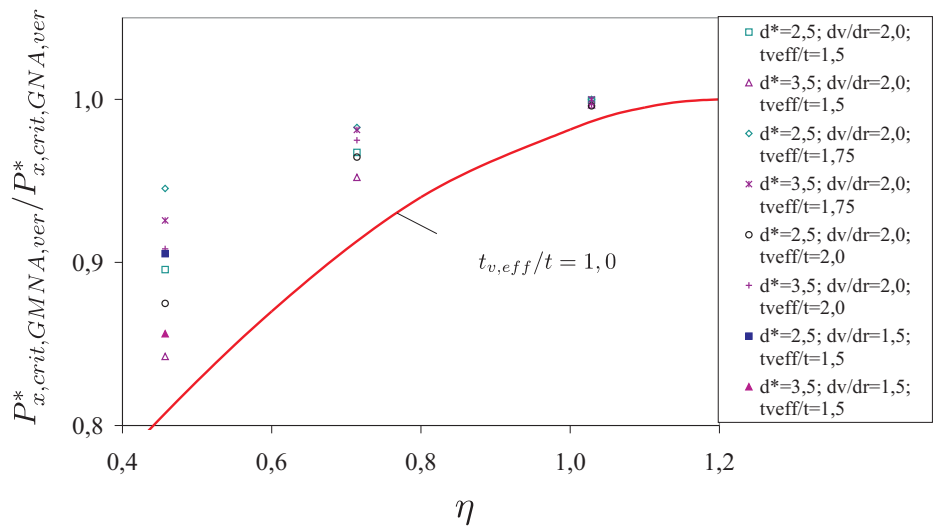

Abbildung 7.25: Verhältniswerte $P_{x, c r i t, G M N A, v e r} / P_{x, c r i t, G N A, v e r}$ der kritischen Lasten von verstärkten Kreiszylinderschalen

\subsection{Kreiszylinderschalen mit Verstärkungsblechen unter Momentenbelastung $M_{\varphi}$ in Umfangsrich- tung}

\subsubsection{Geometrisch lineare Berechnungen mit elastischem Werkstoffgesetz $\left(M_{\varphi}\right.$ ver LA)}

Bei den geometrisch und materiell linearen Untersuchungen der unverstärkten Kreiszylinderschale unter einer Momentenbelastung in Umfangrichtung in Abschnitt 6.5.2 hat sich gezeigt, dass der bezogene Randabstand $L^{*}$ keinen Einfluss auf den Verlauf der Spannungen hat und der Einfluss von $R / t$ vernachlässigt werden kann. Da ein wesentlicher Einfluss dieser Paramter auf den Spannungsverlauf einer verstärkten Kreiszylinderschale ausgeschlossen werden kann, wird bei allen Untersuchungen von einer Kreiszylinderschale mit $R / t=5000$ und $L^{*}=80$ ausgegangen. Desweitern hatte sich in Abschnitt 6.5.2 gezeigt, dass bei Einwirkung eines Moments $M_{\varphi}$ in Umfangsrichtung die Längsspannungen an der Außenseite der Kreiszylinderschale entlang des Meridians die maßgebenden Spannungen 
sind, so dass im Folgenden nur diese Spannungen betrachtet werden. Die Auswirkungen der Verstärkungsblechabmessungen $l_{v}^{*}$ und $t_{v, \text { eff }}$ auf den Spannungsverlauf wurden gemäß dem in Tabelle 7.4 aufgeführten Variationschema untersucht.

\begin{tabular}{|c|c|c|c|c|}
\hline$R / t$ & $L^{*}$ & $d^{*}$ & $t_{v, \text { eff }} / t$ & $l_{v}^{*}$ \\
\hline \hline 5000 & 80 & 3,5 & 2,0 & $0-1,25$ \\
\hline 5000 & 80 & $0,5-3,5$ & 2,0 & 1,25 \\
\hline 5000 & 80 & 3,5 & $1,0-2,0$ & 1,25 \\
\hline
\end{tabular}

Tabelle 7.4: Variationsparameter $\left(M_{\varphi}\right.$ ver LA)

In Abbildung 7.26 sind für eine Kreiszylinderschale mit $R / t=5000, L^{*}=80$ und $d^{*}=3,5$ die normierten Längsspannungen $\sigma_{z, v e r}^{*} / \sigma_{z, \max , M_{\varphi}}^{*}$ an der SchalenauBenseite entlang des Meridians aufgetragen. Dabei wurde die bezogene Länge des Verstärkungsbleches $l_{v}^{*}$ zwischen 1,0 und 1,25 varriiert. Die Blechdickenverhältnisse $t_{v, \text { eff }} / t$ lagen konstant bei 2,0. Es zeigte sich, dass im Übergangsbereiche vom Verstärkungsblech zum unverstärkten Mantelblech (Stelle C und E) die Spannungen in Abhängigkeit von der Länge des Verstärkungsbleches ansteigen. Da in diesem Bereich die Biegespannungen gegenüber den Normalspannungen überwiegen, beträgt die Differenz zwischen den Spannungen am Rande des Verstärkungsbleches (,,B“) und den Spannungen im unverstärkten Mantelblech $\left(, \mathrm{E}^{\star \prime}\right)$ etwa $\left(t_{v, \text { eff }} / t\right)^{2}$. Im dargestellten Fall sind für $l_{v}^{*}<0,3125$ die Spannungen im unverstärkten Mantelblech („,E“) größer als die Spannungen am Stutzenanschluss. Mit zunehmendem $l_{v}^{*}$ nehmen die Spannungen an der Stelle E ab. Für $l_{v}^{*}>1,25$ sind die Spannungen am äußeren Rand des Verstärkungsbleches (Stelle C) soweit abgeklungen, dass der erneute Spannungsanstieg im unverstärkten Mantelblech (Stelle E) sehr gering ist.

Im nächsten Schritt wurde der Einfluss des bezogenen Stutzendurchmessers $d^{*}$ auf das Spannungsverhältnis $\sigma_{z, v e r}^{*} / \sigma_{z, \max , M_{\varphi}}^{*}$ untersucht. Dazu wurde eine Kreiszylinderschale mit $L^{*}=80, R / t=5000, l_{v}^{*}=1,25, t_{v, \text { eff }} / t=2,0$ und veränderlichem $d^{*}$ untersucht. Der Spannungsverlauf, der sich aus diesen Berechnungen ergibt, ist in Abbildung 7.27 aufgetragen. Dabei zeigt sich, dass der Einfluss von $d^{*}$ auf das Spannungsverhältnis $\sigma_{z, v e r}^{*} / \sigma_{z, \max , M_{\varphi}}^{*}$ gering ist und vereinfachend vernachlässigt werden kann. Bei den folgenden Untersuchungen wird daher stets von einer Kreiszylinderschale mit $d^{*}=3,5$ ausgegangen, die den ungünstigsten Fall berücksichtigt. 


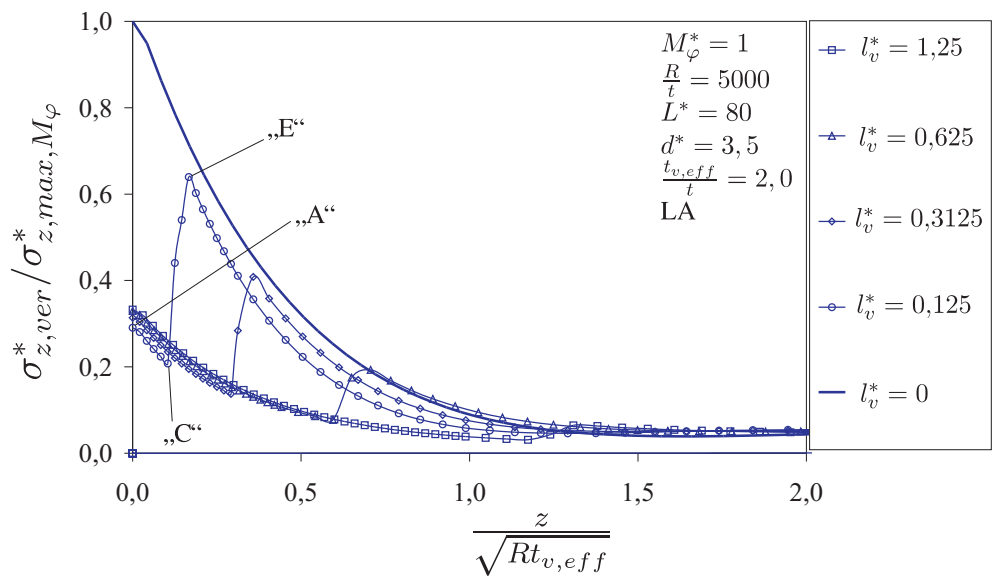

Abbildung 7.26: Spannungsverhältnis $\sigma_{z, v e r}^{*} / \sigma_{z, \max , M_{\varphi}}^{*}$ entlang des Meridians einer Kreiszylinderschale mit $L^{*}=80, d^{*}=3,5$ und einem Verstärkungsblech mit $t_{v, \text { eff }} / t=2,0$ und $0 \leq l_{v}^{*} \leq 1,25$

Bei Variation der Dickenverhältnisse $t_{v, \text { eff }} / t$ des Verstärkungsbleches zeigt sich, dass die Spannungen am Anschluss des Stutzens an das Verstärkungsblech von $t_{v, \text { eff }} / t$ abhängig sind. Dies wird in Abbildung 7.28 am Beispiel einer Kreiszylinderschale mit $L^{*}=80, R / t=5000, d^{*}=3,5$ und einer bezogenen Länge $l_{v}^{*}$ der Verstärkungsbleche von 1,25 gezeigt. 


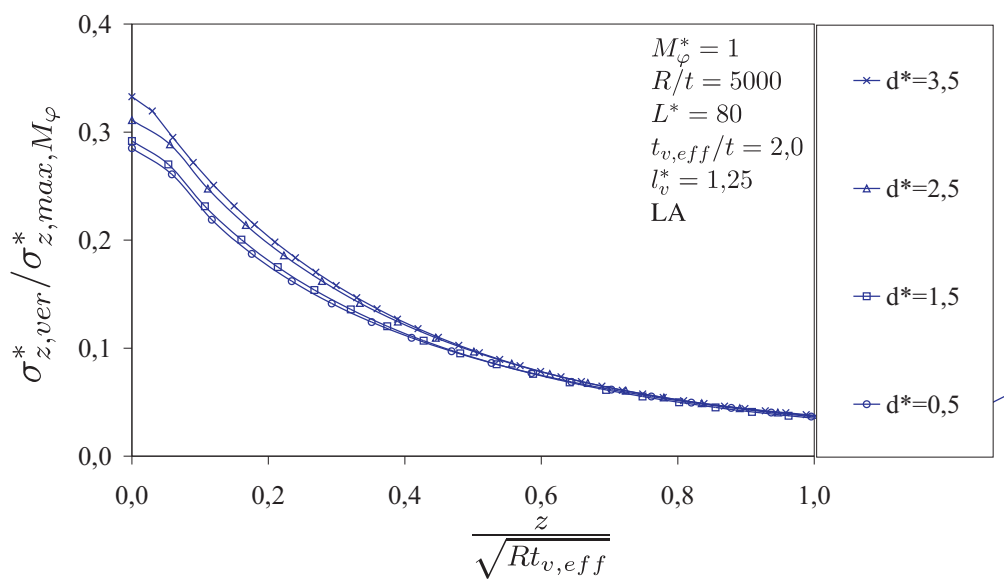

Abbildung 7.27: Spannungsverhältnis $\sigma_{z, v e r}^{*} / \sigma_{z, \max , M_{\varphi}}^{*}$ entlang des Meridians einer Kreiszylinderschale mit $L^{*}=80, R / t=5000$ und $0,5 \leq d^{*} \leq 3,5$ mit einem Versstärkungsblech mit $l_{v}^{*}=1,25$ und $t_{v, \text { eff }} / t=2,0$

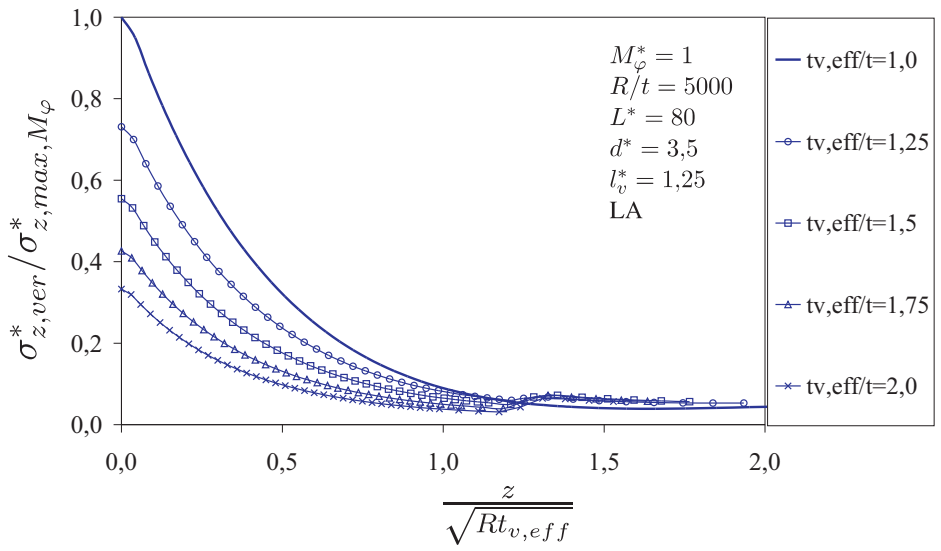

Abbildung 7.28: Spannungsverhältnis $\sigma_{z, v e r}^{*} / \sigma_{z, \max , M_{\varphi}}^{*}$ entlang des Meridians einer Kreiszylinderschale mit $L^{*}=80, d^{*}=3,5, R / t=5000, l_{v}^{*}=1,25$ und $1,0 \leq t_{v, e f f} / t \leq 2,0$ 
Das Verstärkungeblech muss eine Mindestlänge $l_{v, \text { min }}^{*}$ haben, damit die Spannungen im unverstärkten Mantelblech (Stelle E) kleiner sind, als die Spannungen am Stutzenanschluss. In Abhängigkeit von $t_{v, \text { eff }} / t$ kann die dimensionslose Verstärkungsblechlänge $l_{v, \text { min }}^{*}$ mit

$$
l_{v, \text { min }}^{*}=-0,2\left(\frac{t_{v, e f f}}{t}\right)^{2}+\left(\frac{t_{v, \text { eff }}}{t}\right)-0,8
$$

abgeschätzt werden. Das Spannnungsverhältnis $\sigma_{z, v e r}^{*} / \sigma_{z, \max , M \varphi}^{*}$ am Stutzenanschluss, das auftritt, wenn die Mindestlänge $l_{v, \min }^{*}$ der Verstärkungsbleche eingehalten ist, kann mit

$$
\frac{\sigma_{z, v e r}^{*}}{\sigma_{z, \max , M \varphi}^{*}}=0,64\left(\frac{t}{t_{v, \text { eff }}}\right)+0,4\left(\frac{t}{t_{v, \text { eff }}}\right)-0,04
$$

abgeschätzt werden. Mit $d^{*}$ und $l_{v, \text { min }}^{*}$ ergibt sich nach Gleichung 7.7 das erforderliche Durchmesserverhältnis $\left(d_{v} / d_{r}\right)_{\text {min }}$, das aus konstruktiven Gründen auf $d_{v} / d_{r} \leq 3,0$ begrenzt ist. In Abhängigkeit von $t_{v, \text { eff }} / t$ und $d^{*}$ können mit Abbildung 7.29 die Mindestwerte $\left(d_{v} / d_{r}\right)_{\min }$ für die Durchmesserverhältnisse ermittelt werden. Das maximale Spannungsverhältnis $\sigma_{z, v e r}^{*} / \sigma_{z, \text { max }}^{*}$, das bei Einhalten der Mindestdurchmesser am Stutzenanschluss auftritt, kann aus Abbildung 7.30 in Abhängigkeit von $t_{v, e f f} / t$ abgelesen werden.

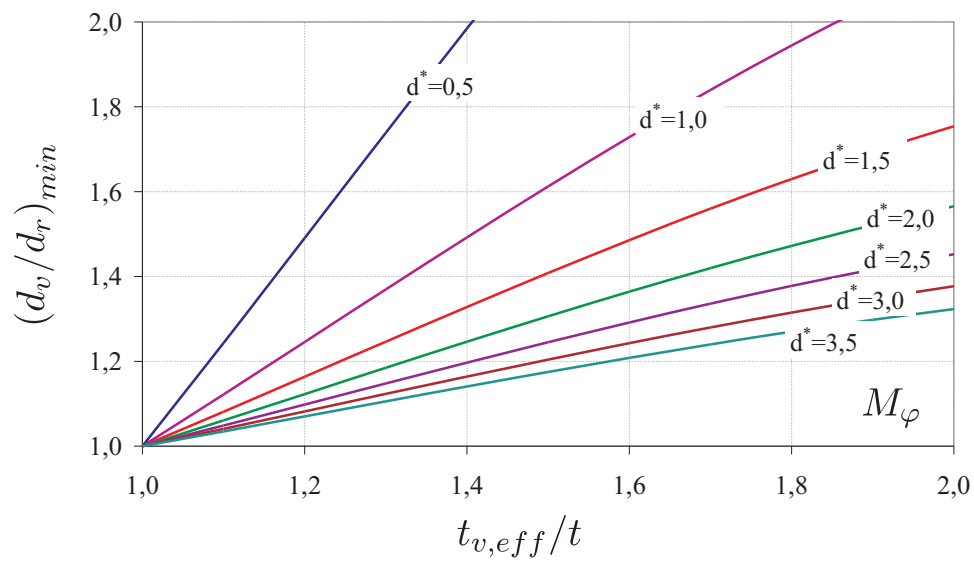

Abbildung 7.29: Durchmesserverhältnis $\left(d_{v} / d_{r}\right)_{\min }$ für den Lastfall $M_{\varphi}$ in Abhängigkeit von $d^{*}$ und $t_{v, e f f} / t$ 


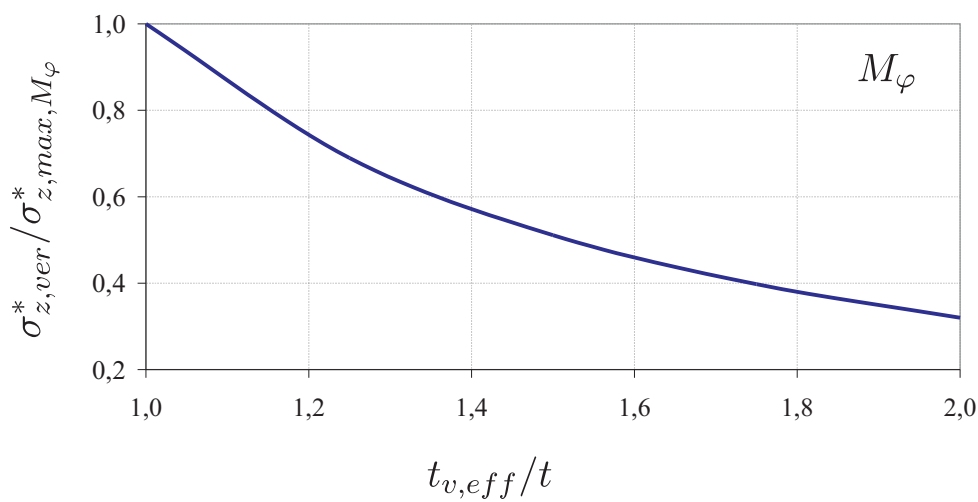

Abbildung 7.30: Spannungsverhältnis $\sigma_{z, v e r}^{*} / \sigma_{z, \max , M_{\varphi}}^{*}$ für den Lastfall $M_{\varphi}$ in Abhängigkeit von $t_{v, \text { eff }} / t$

\subsubsection{Geometrisch nichtlineare Berechnungen mit elastischem Werkstoffgesetz $\left(M_{\varphi}\right.$ ver GNA)}

Wie sich in Abschnitt 6.5.3 gezeigt hat, wird das Tragverhalten der unverstärkten Kreiszylinderschale maßgeblich von $d^{*}$ beeinflusst. Das $R / t$-Verhätnis beeinflusst das Tragverhalten nur im Bereich sehr großer Verformungen, wohingehen sich $L^{*}$ in keinem Bereich auf das Tragverhalten auswirkt. Für verstärkte Kreiszylinderschalen ist kein grundsätzlich anderes Tragverhalten zu erwarten. Die Variationsparameter zur Untersuchung der Einflüsse der Verstärkungsbleche sind in Tabelle 7.5 aufgeführt.

\begin{tabular}{|c|c|c|c|c|}
\hline$R / t$ & $L^{*}$ & $d^{*}$ & $d_{v} / d_{r}$ & $t_{v, \text { eff }} / t$ \\
\hline \hline 400 & 20 & 1,5 & 2,0 & $1,0-2,0$ \\
\hline 400 & 20 & 1,5 & $1,0-2,0$ & 2,0 \\
\hline
\end{tabular}

Tabelle 7.5: Variationsparameter $\left(M_{\varphi}\right.$ ver GNA)

Aus konstruktiven Gründen werden Durchmesserverhältnisse $d_{v} / d_{r}$ von maximal 2,0 und Blechdickenverhältnisse $t_{v, \text { eff }} / t$ bis 2,0 untersucht.

In Abbildung 7.31 sind die Momenten-Rotationskurven von Kreiszylinderschalen mit $L^{*}=20$ und $R / t=400$ dargestellt, deren Verstärkungbleche ein Durch- 
messerverhältnis von $d_{v} / d_{r}=2,0$ und Blechdickenverhältnisse $t_{v, \text { eff }} / t$ zwischen 1,0 und 2,0 haben. Diese Momenten-Rotationskurven veranschaulichen, dass mit zunehmender Verstärkungsblechdicke die Steifigkeit $c_{M_{\varphi, v e r}}^{*}$ der gesamten Kreiszylinderschale zunimmt. Dabei ist auch die Momenten-Rotationskurve einer unverstärken Kreiszylinderschale mit einem bezogenen Stutzendurchmesser $d^{*}$ von 3,0 dargestellt. Diese Kurve entspricht dem Grenzfall eines unendlich steifen Bleches.

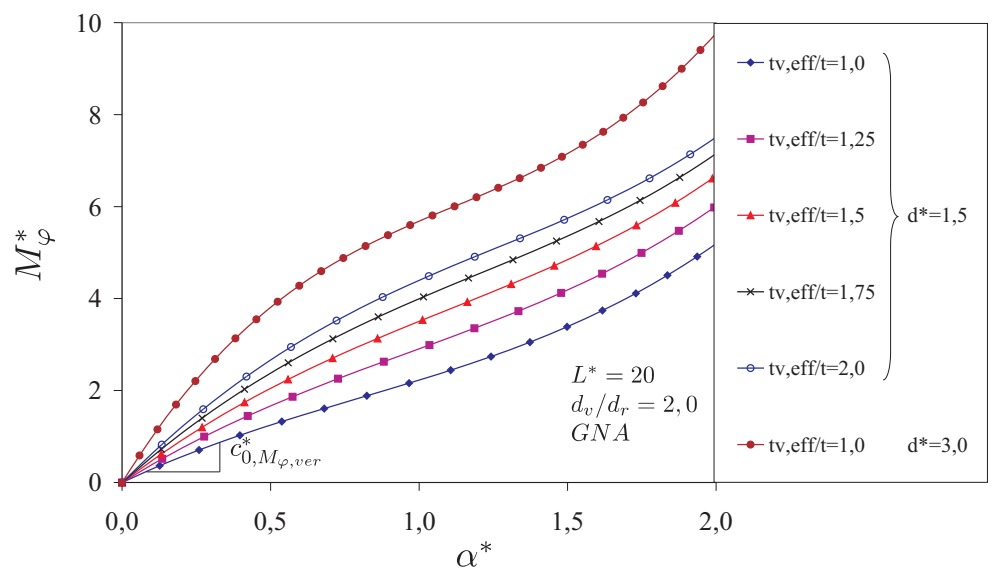

Abbildung 7.31: Momenten-Rotationskurven einer Kreiszylinderschale mit $L^{*}=20, R / t=400$ und $d^{*}=1,5$ mit Verstärkungblechen mit $d_{v} / d_{r}=2,0$ und unterschiedlichen Blechdickenverhältnisse $1,0 \leq t_{v, \text { eff }} / t \leq 2,0$

Im nächsten Schritt wurden Kreiszylinderschalen mit variablen $d_{v} / d_{r}$-Verhältnissen untersucht. Die Steifigkeit $c_{M_{\varphi, v e r}}$ dieser Kreiszylinderschalen mit $L^{*}=20$, $d^{*}=1,5$ und $t_{v, \text { eff }} / t=2,0$ und unterschiedlichen Durchmesserverhältnissen $d_{v} / d_{r}$ nimmt mit zunehmendem $d_{v} / d_{r}$ zu, wie anhand der MomentenRotationskurven in Abbildung 7.32 verdeutlicht wird.

In Serienberechnungen wurden die kritischen Momente $M_{\varphi, c r i t, G N A, v e r}^{*}$ in Abhängigkeit von $d^{*}, d_{v} / d_{r}$ und $t_{v, \text { eff }} / t$ ermittelt. Da die MomentenRotationskurve von Kreiszylinderschalen, deren bezogene Stutzendurchmesser $d^{*}$ kleiner als 3,5 sind, keine lokalen Maxima haben, werden die kritischen Momente $M_{\varphi, c r i t, G N A, v e r}$ wie in Abschnitt 6.5.3 für die unverstärkte Kreiszylinderschale beschrieben, durch Berechnung einer Kreiszylinderschale mit $d^{*}=5,0$ bestimmt. Das kritische Moment $M_{\varphi, c r i t, G N A, v e r}^{*}$ ist in Abbildung 7.33 für Kreis- 


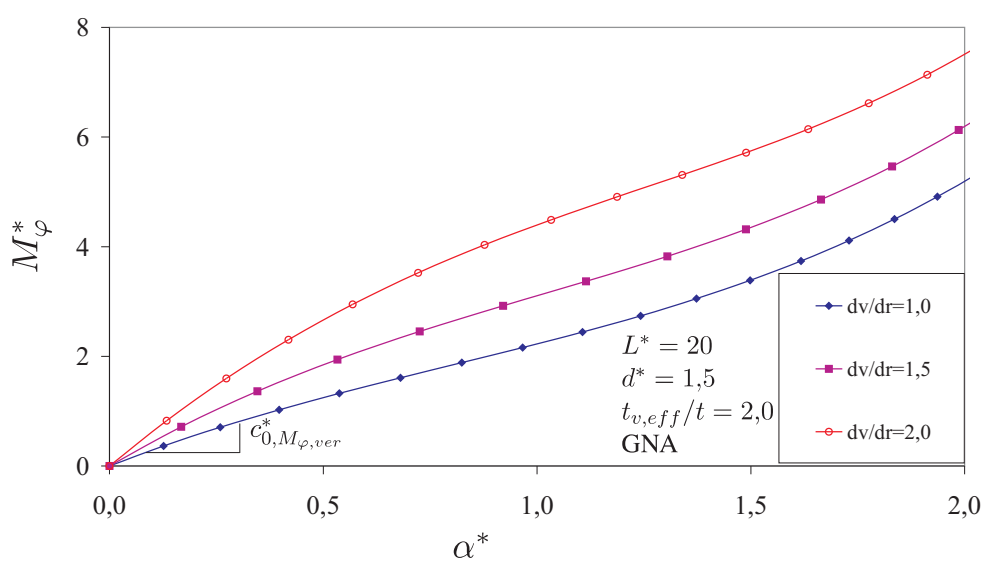

Abbildung 7.32: Momenten-Rotationskurven einer Kreiszylinderschale mit $L^{*}=20, R / t=400$ und $d^{*}=1,5$ mit Verstärkungblechen mit $d_{v} / d_{r}=2,0$ und unterschiedlichen Blechdickenverhältnisse $1,0 \leq t_{v, \text { eff }} / t \leq 2,0$

zylinderschalen mit $d_{v} / d_{r}=1,5$ und in Abbildung 7.34 für Kreiszylinderschalen mit $d_{v} / d_{r}=2,0$ über $d^{*}$ für unterschiedliche $t_{v, \text { eff }} / t$ aufgetragen.

Die Anfangssteifigkeit $c_{M_{\varphi, v e r}}$ der verstärkten Kreiszylinderschale unter Einwirkung von $M_{\varphi}$ ist ebenfalls abhängig von $d^{*}, t_{v, \text { eff }} / t$ und $d_{v} / d_{r}$. In Abbildung 7.35 ist $c_{M_{\varphi, v e r}}^{*}$ für Verstärkungsbleche mit $d_{v} / d_{r}=1,5$ und in Abbildung 7.36 für Verstärkungsbleche mit $d_{v} / d_{r}=2,0$ für unterschiedliche $t_{v, \text { eff }} / t$ über $d^{*}$ aufgetragen. 


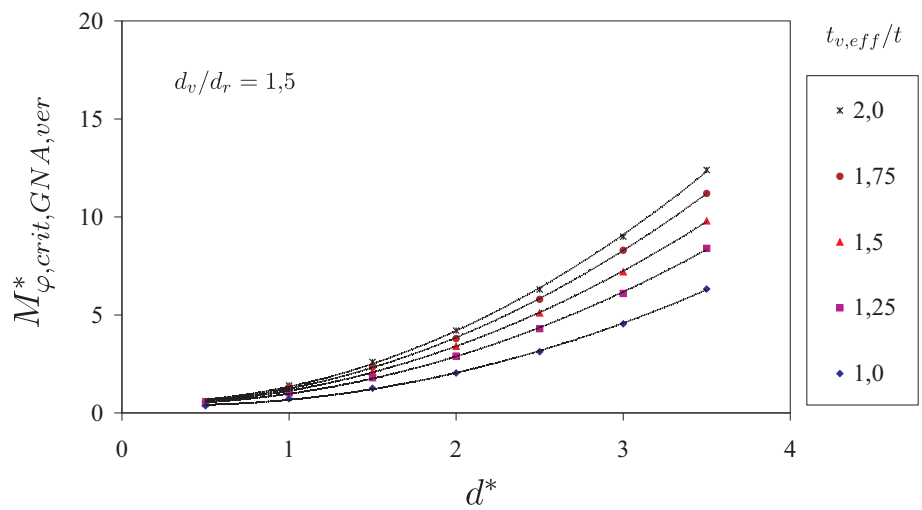

Abbildung 7.33: Kritisches Moment $M_{\varphi, c r i t, G N A, v e r}^{*}$ in Abhängigkeit von $d^{*}$ und $t_{v, \text { eff }} / t$ für Kreiszylinderschalen mit Verstärkungblechen mit $d_{v} / d_{r}=1,5$ und $1,0 \leq t_{v, e f f} / t \leq 2,0$

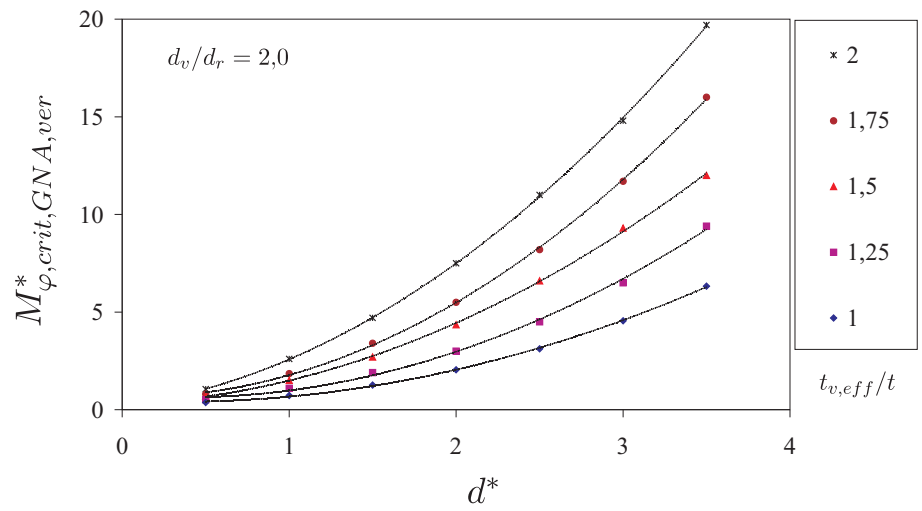

Abbildung 7.34: Kritisches Moment $M_{\varphi, c r i t, G N A, v e r}^{*}$ in Abhängigkeit von $d^{*}$ und $t_{v, \text { eff }} / t$ für Kreiszylinderschalen mit Verstärkungblechen mit $d_{v} / d_{r}=2,0$ und $1,0 \leq t_{v, \text { eff }} / t \leq 2,0$ 


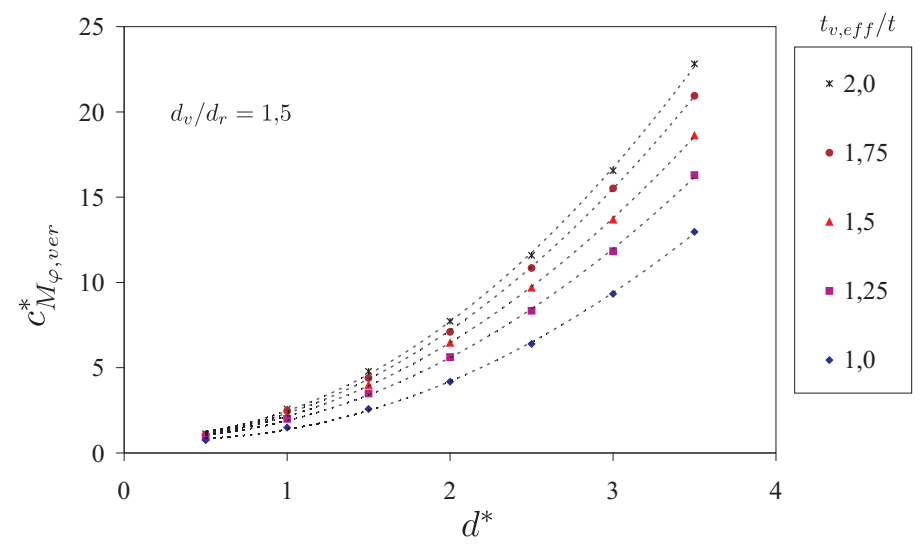

Abbildung 7.35: Anfangssteifigkeit $c_{M_{\varphi, v e r}}^{*}$ in Abhängigkeit von $d^{*}$ und $t_{v, \text { eff }} / t$ für Kreiszylinderschalen mit Verstärkungblechen mit $d_{v} / d_{r}=1,5$ und $1,0 \leq t_{v, \text { eff }} / t \leq 2,0$

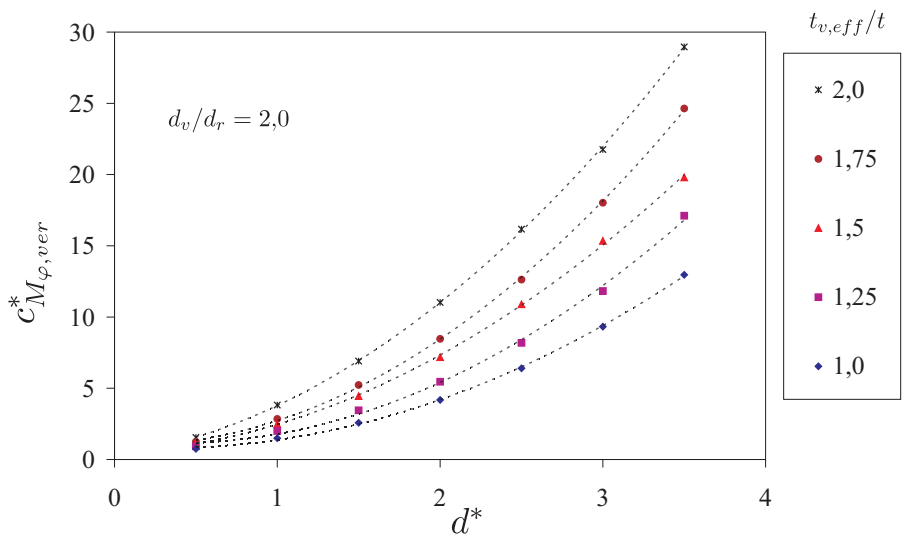

Abbildung 7.36: Anfangssteifigkeit $c_{M_{\varphi, v e r}}^{*}$ in Abhängigkeit von $d^{*}$ und $t_{v, \text { eff }} / t$ für Kreiszylinderschalen mit Verstärkungblechen mit $d_{v} / d_{r}=2,0$ und $1,0 \leq t_{v, \text { eff }} / t \leq 2,0$ 


\subsubsection{Einfluss materieller Nichtlinearität auf das Tragverhal- ten verstärkter Kreiszylinderschalen}

Bei Berücksichtigung des plastischen Werkstoffverhaltens in den numerischen Berechnungen zeigt sich der Einfluss des plastischen Faktors $\eta$ auf das Tragverhalten. Bei der verstärkten Kreiszylinderschale führen zudem unterschiedliche Blechdickenverhältnisse $t_{v, \text { eff }} / t$ und Duchmesserverhältnisse $d_{v} / d_{r}$ zu unterschiedlichen kritischen Momenten. Anhand von Parameterstudien mit den in Tabelle 7.6 aufgeführten Kreiszylinderschalen wurden diese Einflüsse untersucht.

\begin{tabular}{|c|c|c|c|c|c|}
\hline$R / t$ & $L^{*}$ & $d^{*}$ & $f_{y}$ & $d_{v} / d_{r}$ & $t_{v, \text { eff }} / t$ \\
\hline \hline 400 & 20 & 1,5 & $240-690$ & 2,0 & 2,0 \\
\hline 400 & 20 & 1,5 & 240 & $1,0-2,0$ & 2,0 \\
\hline 400 & 20 & 1,5 & 240 & 2,0 & $1,0-2,0$ \\
\hline
\end{tabular}

Tabelle 7.6: Variationsparameter $\left(M_{\varphi}\right.$ ver GMNA)

Die Ergebnisse der Untersuchungsschritte 1 und 2 sind in Abbildung 7.37 dargestellt. Dabei sind die Momenten-Rotationskurven von Kreiszylinderschalen mit veränderlichem $f_{y}$ und $t_{v, \text { eff }} / t$ aufgetragen. In Abbildung 7.38 sind die Momenten-Rotationskurven von Kreiszylinderschalen mit veränderlichem $f_{y}$ und $d_{v} / d_{r}$ dargestellt. Da das $R / t$-Verhältnis jeweils konstant und die Streckgrenze $f_{y}$ variabel ist, haben diese Momenten-Rotationskurven variable $\eta$.

Aus beiden Abbildungen ist ersichtlich, dass das Plastizieren der Verstärkungsbleche zu einer Abminderung der Tragfähigkeit führt. Wie für den Lastfall $P_{x}$ in Abschnitt 7.2.3 bereits gezeigt wurde, ist die Abminderung infolge des plastischen Werkstoffverhaltens von einer Vielzahl von Parametern abhängig. Im ungünstigsten Fall, wenn $t_{v, \text { eff }} / t=1,0$ ist, beträgt die Abminderung $80 \%$. Diese Sachverhalte gelten auch für den Lastfall $M_{\varphi}$, wobei die maximale Abminderung im untersuchten Parameterbereich maximal $60 \%$ beträgt. Vereinfachend und auf der sicheren Seite liegend können daher die Abminderungskurven der unverstärkten Kreiszylinderschalen aus Abbildung 6.40 für das kritische Moment $M_{\varphi, c r i t, G M N A, v e r}^{*}$ angewandt werden. 


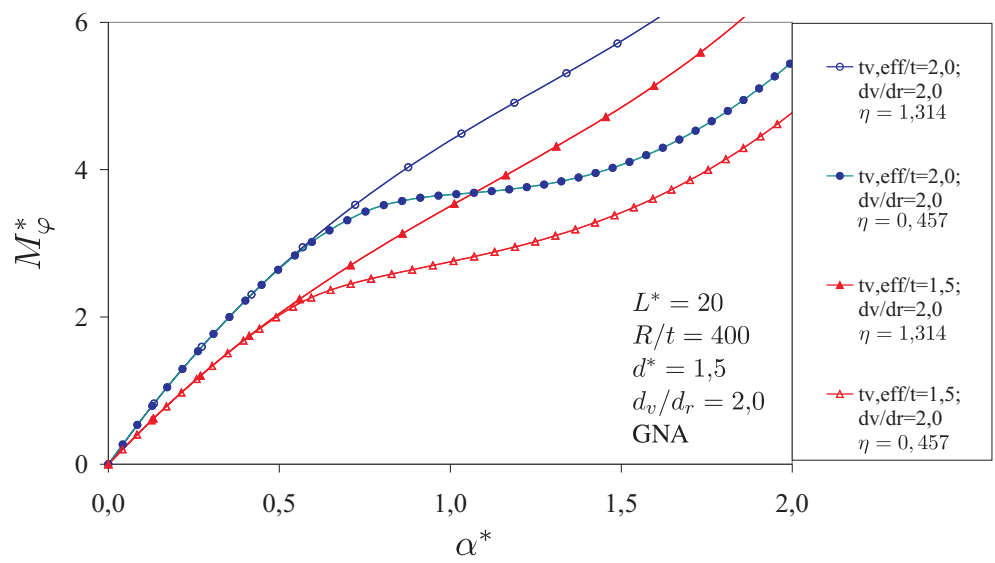

Abbildung 7.37: Momenten-Rotationskurven einer Kreiszylinderschale mit $L^{*}=20, R / t=400$ und $d^{*}=1,5$ mit Verstärkungblechen mit $d_{v} / d_{r}=2,0$ und unterschiedlichen Blechdickenverhältnisse $1,0 \leq t_{v, \text { eff }} / t \leq 2,0$

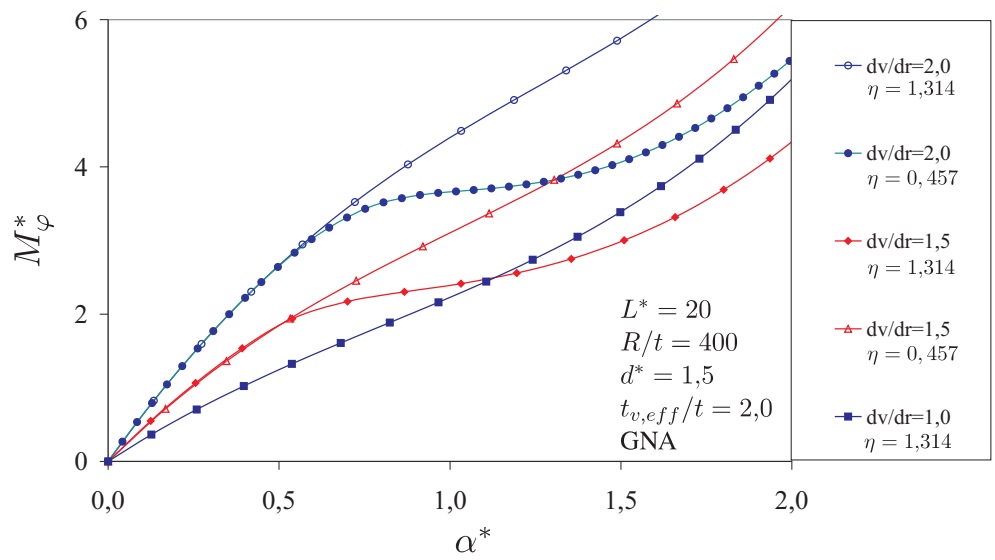

Abbildung 7.38: Momenten-Rotationskurven einer Kreiszylinderschale mit $L^{*}=20, R / t=400$ und $d^{*}=1,5$ mit Verstärkungblechen mit $t_{v, \text { eff }} / t=2,0$ und unterschiedlichen Blechdickenverhältnisse $1,0 \leq d_{v} / d_{r} \leq 2,0$ 


\subsection{Kreiszylinderschalen mit Verstärkungsblechen unter Momentenbelastung $M_{z}$ in Meridianrich- tung}

\subsubsection{Geometrisch lineare Berechnungen mit elastischem Werkstoffgesetz $\left(M_{z}\right.$ ver LA)}

Bei Kreiszylinderschalen, die durch ein Moment $M_{z}$ in Meridianrichtung beansprucht werden, sind die maximalen Spannungen die Umfangsspannungen $\sigma_{\varphi}^{*}$ in Umfangsrichtung. Die Längsspannungen sind gegenüber den Umfangsspannungen sehr klein und werden folglich nicht betrachtet. Dies wurde in Abschnitt 6.6.2 für die unverstärkte Kreiszylinderschale gezeigt und trifft auch für die verstärkte Kreiszylinderschale zu. Außerdem wurde in Abschnitt 6.6.2 gezeigt, dass der bezogene Randabstand $L^{*}$ keinen Einfluss auf das Tragverhalten hat. Das $R / t$ Verhältnis hat nur einen geringen Einfluss auf den Spannungsverlauf. In den folgenden Untersuchungen werden die Einflüsse dieser Paramter vernachlässigt. Die Konstanten und Variablen der einzelnen Untersuchungsschritte sind in Tabelle 7.7 aufgeführt.

\begin{tabular}{|c|c|c|c|c|}
\hline$R / t$ & $L^{*}$ & $d^{*}$ & $t_{v, \text { eff }} / t$ & $l_{v}^{*}$ \\
\hline \hline 5000 & 80 & 3,5 & 2,0 & 4,89 \\
\hline 5000 & 80 & $0,5-3,5$ & 2,0 & 4,89 \\
\hline 5000 & 80 & 3,5 & $1,0-2,0$ & 4,89 \\
\hline
\end{tabular}

Tabelle 7.7: Variationsparameter $\left(M_{\varphi}\right.$ ver LA)

In Abbildung 7.39 wurde das Verhältnis der Umfangsspannungen $\sigma_{\varphi, v e r}^{*}$ der verstärkten Kreiszylinderschale zur maximalen Umfangsspannung $\sigma_{\varphi, \text { max }, M_{z}}^{*}$ der unverstärkten Kreiszylinderschale für eine Kreiszylinderschale mit $L^{*}=80$, $R / t=5000$ und $d^{*}=3,5$ entlang des Umfangs aufgetragen. Die eingesetzten Verstärkungsbleche haben ein Blechdickenverhältnis von $t_{v, \text { eff }} / t=2,0$. Die Länge des Verstärkungsbleches $l_{v}^{*}$ wurde dabei zwischen 0 und 4,89 variiert. Dabei zeigt sich deutlich, dass die Spannungen im Verstärkungsblech schnell abklingen und in Abhängigkeit von $l_{v}^{*}$ am Übergang zum unverstärkten Mantelblech wieder ansteigen. Für $l_{v}^{*}>4,89$ sind die Spannungen am äußeren Rand (Stelle D) des Verstärkungsbleches soweit abgeklungen, dass die erneute Spannungszunahme im unverstärkten Mantelblech (Stelle F) sehr gering ist. 


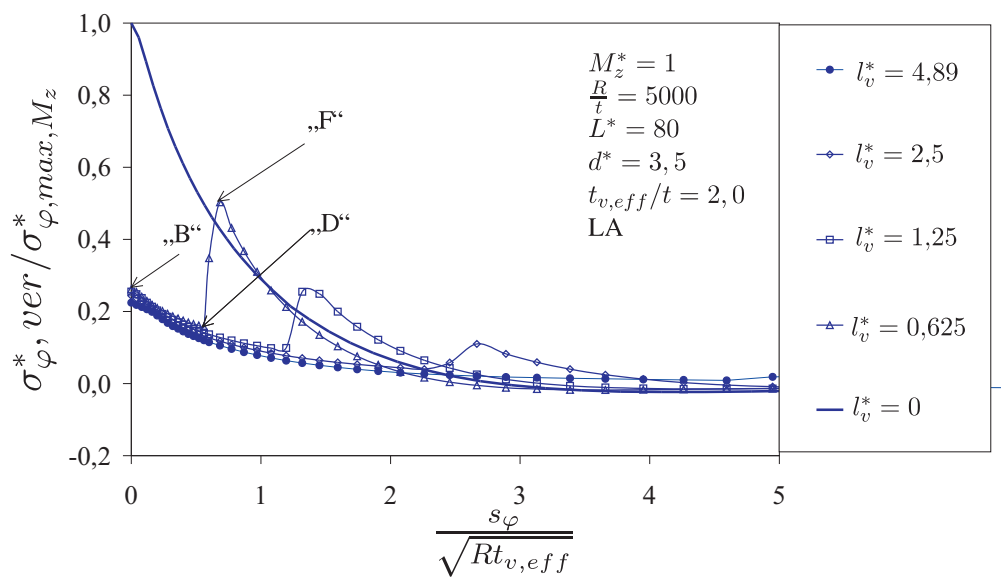

Abbildung 7.39: Spannungsverhältnis $\sigma_{\varphi, v e r}^{*} / \sigma_{\varphi, \max , M_{z}}^{*}$ entlang des Umfangs einer Kreiszylinderschale mit $L^{*}=80, R / t=5000, d^{*}=3,5$ mit $t_{v, \text { eff }} / t=2,0$ und $0<l_{v}^{*} \leq 4,89$

Im nächsten Schritt wurde der bezogene Stutzendurchmesser $d^{*}$ einer Kreiszylinderschale mit konstantem $L^{*}=80, R / t=5000, t_{v, \text { eff }} / t=4,89$ und $d_{v} / d_{r}=2,0$ variiert. In Abbildung 7.40 sind die Spannungsverläufe dieser Kreiszylinderschalen aufgetragen. Dabei zeigt sich, dass unterschiedliche $d^{*}$ nur einen sehr kleinen Einfluss auf das Spannungsverhältnis $\sigma_{\varphi, v e r}^{*} / \sigma_{\varphi, \max , M_{z}}^{*}$ haben und im Folgenden vernachlässigt werden können.

Das Spannungsverhältnis $\sigma_{\varphi, v e r}^{*} / \sigma_{\varphi, \max , M_{z}}^{*}$ am Stutzenanschluss wird maßgeblich vom Blechdickenverhältnis $t_{v, \text { eff }} / t$ beeinflusst, wie Abbildung 7.41 am Beispiel einer Kreiszylinderschale mit $L^{*}=80, R / t=5000, d^{*}=3,5$ und einer Verstärkungsblechlänge $l_{v}^{*}$ von 4,89 zeigt. Mit zunehmendem $t_{v, \text { eff }} / t$ werden die Spannungen am Stutzenanschluss geringer. 


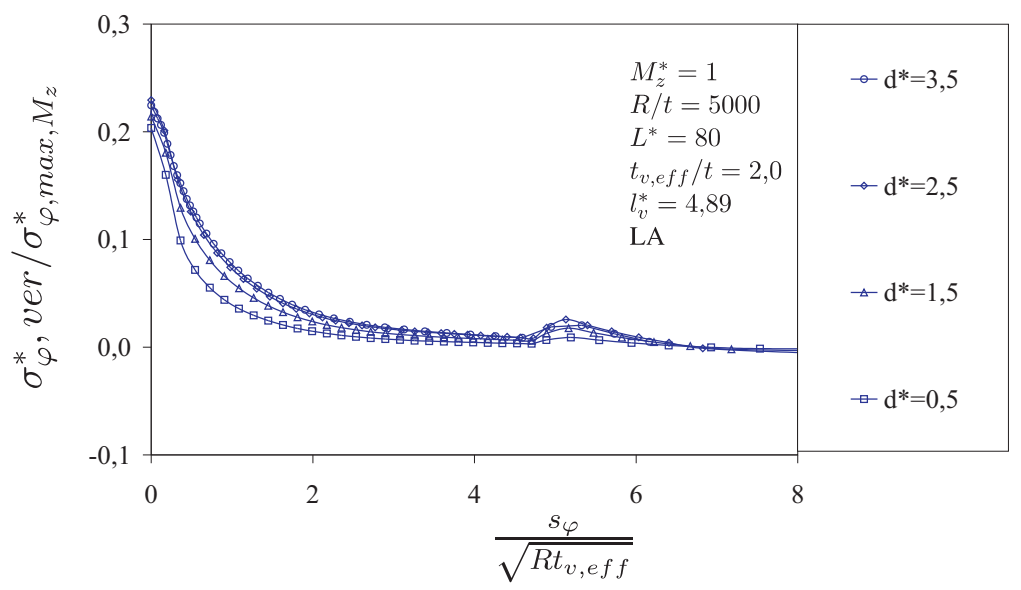

Abbildung 7.40: Spannungsverhältnis $\sigma_{\varphi, v e r}^{*} / \sigma_{\varphi, \max , M_{z}}^{*}$ entlang des Umfangs einer Kreiszylinderschale mit $L^{*}=80, R / t=5000$ und unterschiedlichen $d^{*}$, mit einem Verstärkungsblech mit $t_{v, \text { eff }} / t=2,0$ und $l_{v}^{*}=4,89$

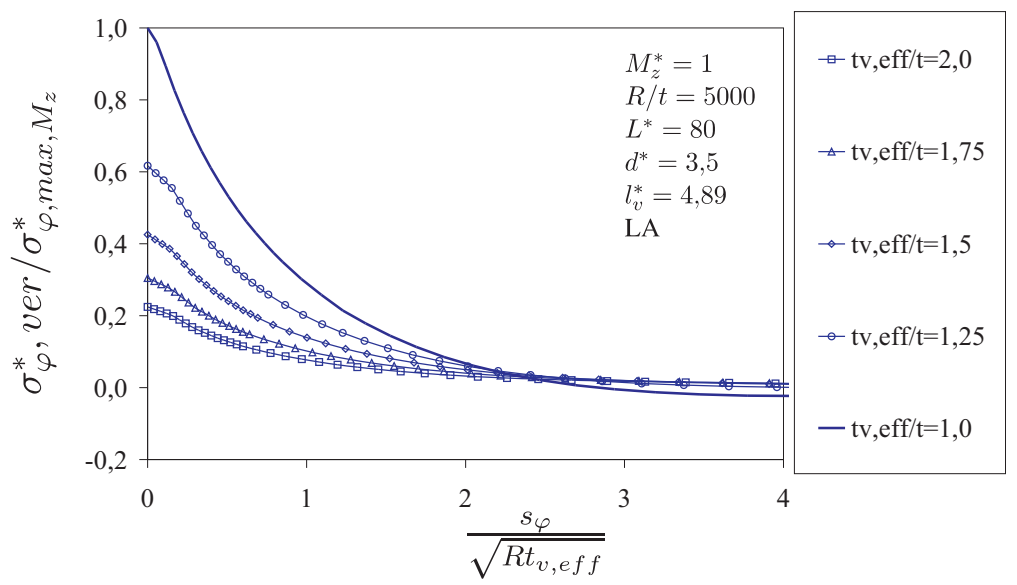

Abbildung 7.41: Spannungsverhältnis $\sigma_{\varphi, v e r}^{*} / \sigma_{\varphi, \max , M_{z}}^{*}$ entlang des Umfangs einer Kreiszylinderschale mit $L^{*}=80, R / t=5000, d^{*}=3,5$ mit $t_{v, \text { eff }} / t=2,0$ und $0<l_{v}^{*} \leq 4,89$ 
Die Abmessungen des Verstärkungsbleches werden so gewählt, dass die Spannungen im Übergang zum unverstärkten Mantelblech (Stelle F) kleiner sind als die Spannungen am Stutzenanschluss (Stelle B). Dies wird erreicht, indem die Verstärkungsbleche mit einer Mindestlänge $l_{v, \text { min }}^{*}$ ausgeführt werden. Diese Mindestlänge kann mit

$$
l_{v, \text { min }}^{*}=0,9\left(\frac{t_{v, \text { eff }}}{t}\right)^{2}-1,5 \frac{t_{v, \text { eff }}}{t}+0,6
$$

abgeschätzt werden kann. Das maximale Spannungsverhältnis am Stutzenanschluss und im unverstärkten Blech kann dabei mit

$$
\frac{\sigma_{\varphi, v e r, M z}^{*}}{\sigma_{\varphi, \max }^{*}}=1,73\left(\frac{t_{v, e f f}}{t}\right)-0,73
$$

überschlägig bestimmt werden. In Abbildung 7.42 ist dieses Spannungsverhältnis $\sigma_{\varphi, v e r}^{*} / \sigma_{\varphi, \max , M_{z}}^{*}$ in Abhängigkeit von $t_{v, \text { eff }} / t$ aufgetragen.

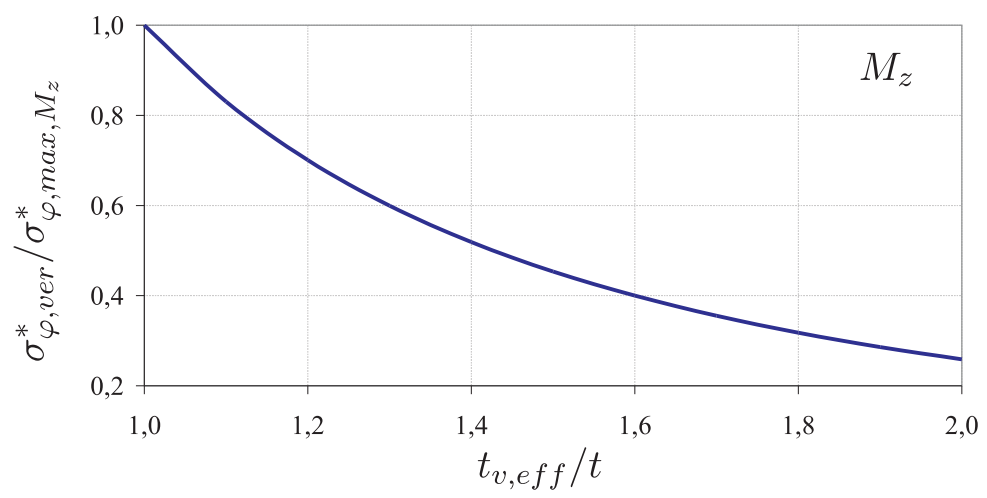

Abbildung 7.42: Spannungsverhältnis $\sigma_{\varphi, v e r}^{*} / \sigma_{\varphi, \max , M_{z}}^{*}$ in Anhängigkeit von $t_{v, \text { eff }} / t$ für den Lastfall $M_{z}$

Mit der Umrechnung der Mindestlänge $l_{v}^{*}$ in das Durchmesserverhältnis $d_{v} / d_{r}$ nach Gleichung 7.7 und der Beschränkung, dass $d_{v} / d_{r} \leq 2,0$ ist, ergibt sich das Mindestdurchmesserverhältnis $\left(d_{v} / d_{r}\right)_{\min }$ in Abhängigkeit von $d^{*}$ und $t_{v, \text { eff }} / t$ nach Abbildung 7.43. 


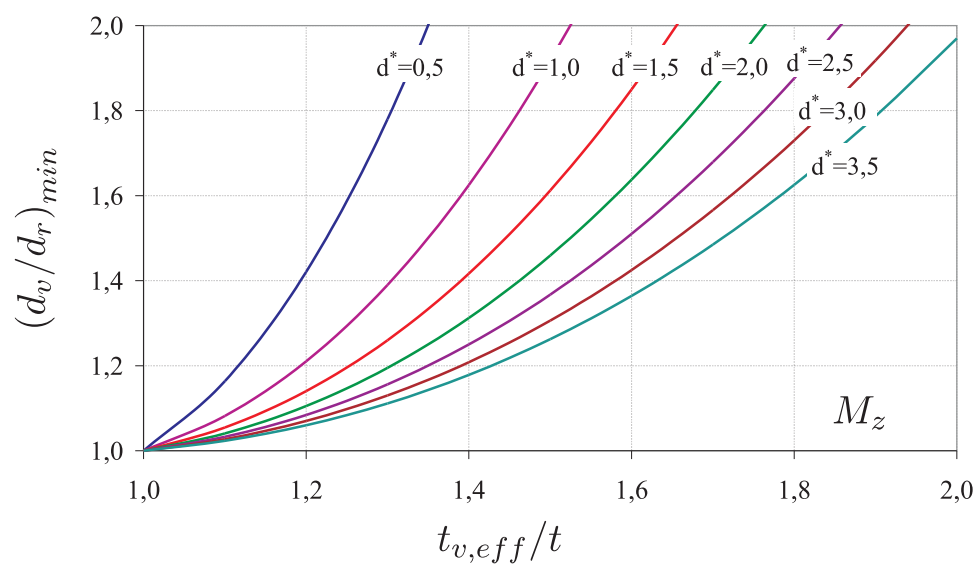

Abbildung 7.43: Durchmesserverhältnis $\left(d_{v} / d r\right)_{\min }$ für den Lastfall $M_{z}$ in Abhängigkeit von $d^{*}$ und $t_{v, \text { eff }} / t$

\subsubsection{Geometrisch nichtlineare Berechnungen mit elastischem Werkstoffgesetz ( $M_{z}$ ver GNA)}

In diesem Abschnitt werden nur Kreiszylinderschalen untersucht, deren Verstärkungsbleche Durchmesserverhältnisse $d_{v} / d_{r}$ zwischen 1,0 und 2,0 und Blechdickenverhältnisse $t_{v, \text { eff }} / t$ zwischen 1,0 und 2,0 haben. Zunächst wurde nur der Einfluss dieser beiden Parameter nach dem in Tabelle 7.8 angegebenen Variationsschema untersucht.

\begin{tabular}{|c|c|c|c|c|}
\hline$R / t$ & $L^{*}$ & $d^{*}$ & $d_{v} / d_{r}$ & $t_{v, e f f} / t$ \\
\hline \hline 400 & 20 & 1,5 & 2,0 & $1,0-2,0$ \\
\hline 400 & 20 & 1,5 & $1,0-2,0$ & 2,0 \\
\hline
\end{tabular}

Tabelle 7.8: Variationsparameter $\left(M_{z}\right.$ ver GNA)

Wie Abbildung 7.44 am Beispiel einer Kreiszylinderschale mit $R / t=400$, $L^{*}=20, d^{*}=1,5$ und Verstärkungblechen mit $d_{v} / d_{r}=2,0$ und $t_{v, \text { ef f }} / t$ zwischen 1,0 und 2,0 zeigt, wirkt sich die lokale Steifigkeitszunahme im Stutzenbereich auch auf das globale Tragverhalten der Kreiszylinderschale aus. Mit zunehmendem $t_{v, e f f} / t$-Verhältnis steigt die Anfangssteifigkeit $c_{M_{z, v e r}}$ der Kreiszylinderschalen. Eine Kreiszylinderschale mit einem vollkommen steifen Lasteinleitungs- 
bereich entspricht einer unverstärkten Kreiszylinderschale, deren Stutzen einen bezogenen Stutzendurchmesser $d^{*}=3,0$ hat. Diese Kurve stellt den oberen Grenzfall dar. Ein Stabilitätsproblem tritt im untersuchten Parameterbereich für diesen Lastfall nicht auf.

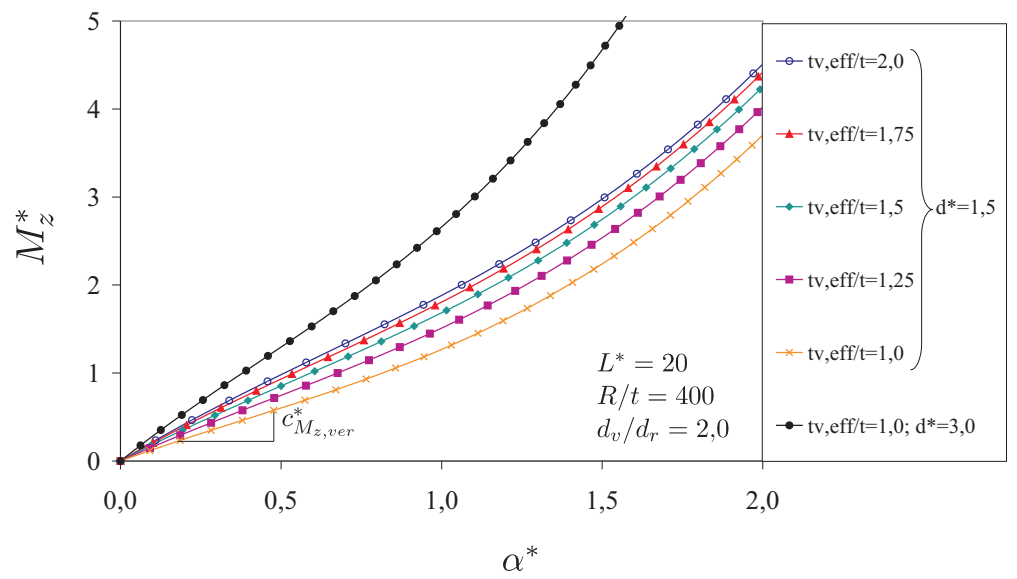

Abbildung 7.44: Momenten-Rotationskurve einer Kreiszylinderschale mit $R / t=400, L^{*}=20, d^{*}=1,5$ mit Verstärkungblechen mit $d_{v} / d_{r}=2,0$ und $t_{v, \text { eff }} / t$ zwischen 1,0 und 2,0

Im nächsten Schritt wurde das Durchmesserverhältnis $d_{v} / d_{r}$ variiert. Die Momenten-Rotationskurven einer Kreiszylinderschale mit $R / t=400, L^{*}=20$, $d^{*}=1,5$ und einem Verstärkungsblech mit $t_{v, \text { eff }} / t=2,0$ und unterschiedlichen $d_{v} / d_{r}$ sind in Abbildung 7.45 dargestellt. Es zeigt sich, dass $d_{v} / d_{r}$ einen wesentlichen Einfluss auf das Tragverhalten der verstärkten Kreiszylinderschale hat.

Da im untersuchten Bereich im Lastfall $M_{z}$ kein Stabilitätsproblem auftritt, wird als Abgrenzungskriterium die dimensionslose Grenzverdrehung $\alpha^{*}=1,0$ der unverstärkten Kreiszylinderschale herangezogen (vgl. Abschnitt 6.6.3). Die kri-

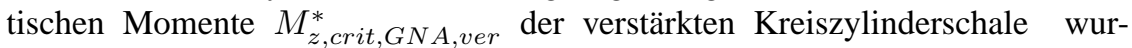
de gemäß Abschnitt 6.3 bestimmt. Dabei zeigt sich, dass $M_{z, c r i t, G N A, v e r}^{*}$ in Abhängigkeit von $d_{v} / d_{r}$ und $t_{v, \text { eff }} / t$ zunimmt.

Wie die Untersuchungen an der unverstärkten Kreiszylinderschale in Abschnitt 6.6.3 gezeigt haben, wirken sich der bezogene Randabstand $L^{*}$ und der Stutzendurchmesser $d^{*}$ auf das Tragverhalten der verstärkten Kreiszylinderschale aus. Der Einfluss des $R / t$-Verhältnisses auf den Anfangsbereich der MomentenRotationskurven spielt dagegen keine Rolle und kann vernachlässigt werden. 


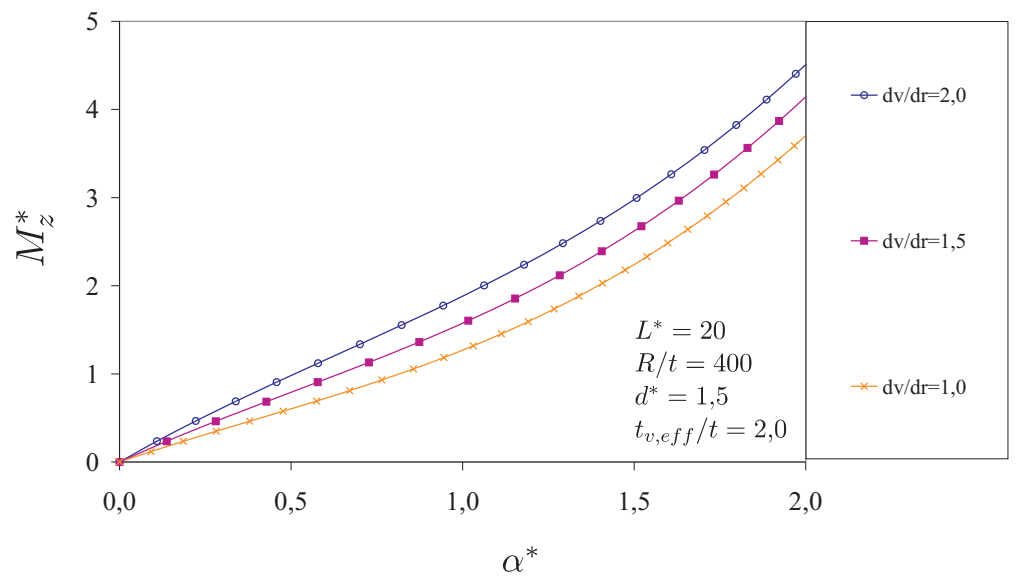

Abbildung 7.45: Momenten-Rotationskurve einer Kreiszylinderschale mit $R / t=400, L^{*}=20, d^{*}=1,5$ mit Verstärkungblechen mit $t_{v, \text { eff }} / t=2,0$ und $d_{v} / d_{r}$ zwischen 1,0 und 2,0

Da diese Sachverhalte auch für verstärkte Kreiszylinderschalen zutreffen, ist das kritische Moment $M_{z, \text { crit }, G N A, v e r}^{*}$ zudem abhängig von $L^{*}$ und $d^{*}$. Für $t_{v, \text { eff }} / t=2,0$ und $d_{v} / d_{r}=2,0$ sind in Abbildung 7.46 beispielhaft die kritischen Momente $M_{z, \text { crit }, G N A, v e r}^{*}$ ür unterschiedliche $d^{*}$ über $L^{*}$ aufgetragen.

Das kritische Moment $M_{z, \text { crit }, G N A, v e r}^{*}$ kann innerhalb des untersuchten Bereichs als Funktion von $L^{*}, d^{*}, t_{v, e f f} / t$ und $d_{v} / d_{r}$ mit

$$
M_{z, \text { crit }, G N A, v e r}^{*}=M_{z, 0}^{*}+\frac{\xi}{L^{*}}
$$

abgeschätzt werden. Dabei können die Werte $M_{z, 0}^{*}$ aus Abbildung 7.47 und $\xi$ aus Abbildung 7.48 für Kreiszylinderschalen mit $d_{v} / d_{r}=1,5$ entnommen werden. Für Kreiszylinderschalen mit $d_{v} / d_{r}=2,0$ kann $M_{z, 0}^{*}$ aus Abbildung 7.49 und $\xi$ aus Abbildung 7.50 abgelesen werden. Für $d_{v} / d_{r}$-Verhältnisse, die zwischen diesen Werte liegen, kann bei der Ermittlung von $M_{z, c r i t, G N A, v e r}^{*}$ linear interpoliert werden. 


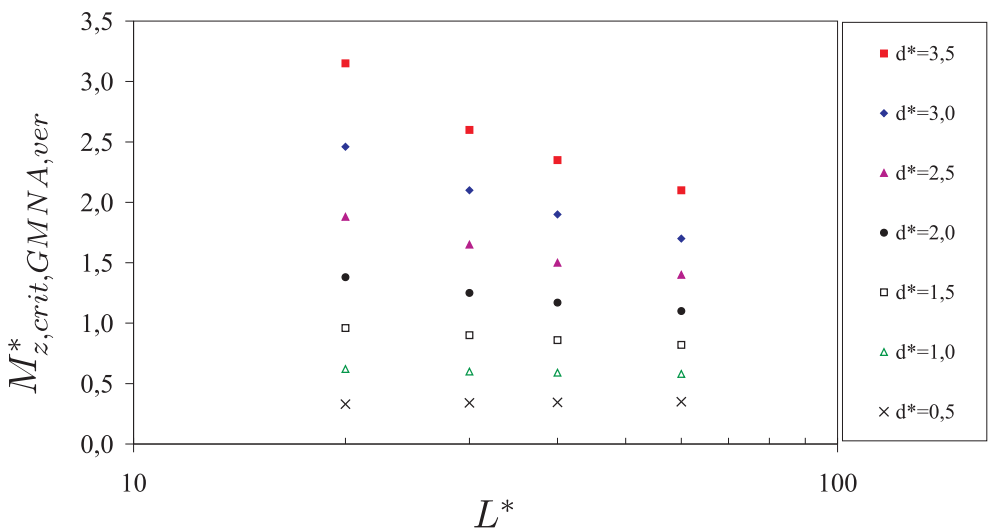

Abbildung 7.46: Kritisches Moment $M_{z, c r i t, G N A, v e r}^{*}$ von Kreiszylinderschalen mit Verstärkungsblechen mit $t_{v, \text { eff }} / t=2,0$ und $d_{v} / d_{r}=2,0$

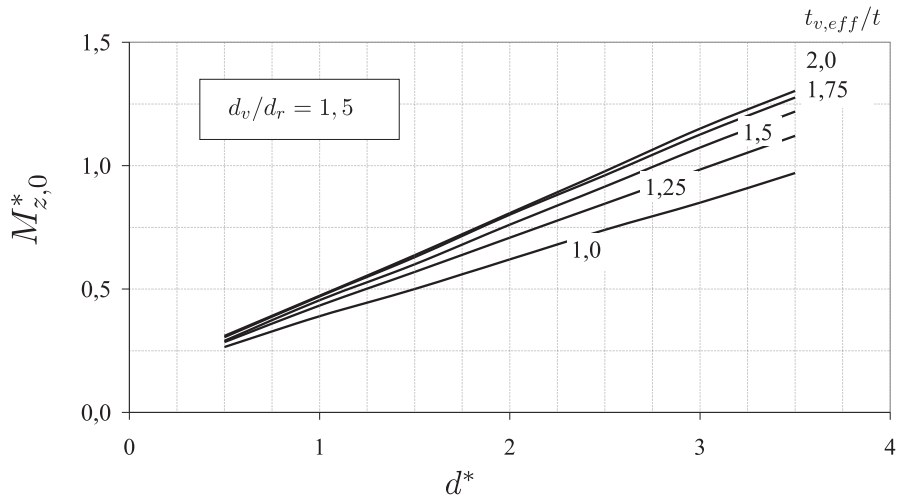

Abbildung 7.47: $M_{z, 0}^{*} \quad$ zur Bestimmung des kritischen Momentes $M_{z, \text { crit }, G N A, \text { ver }}^{*}$ von Kreiszylinderschalen mit Verstärkungsblechen mit $1,0 \leq t_{v, \text { eff }} / t \leq 2,0$ für $d_{v} / d_{r}=1,5$ 


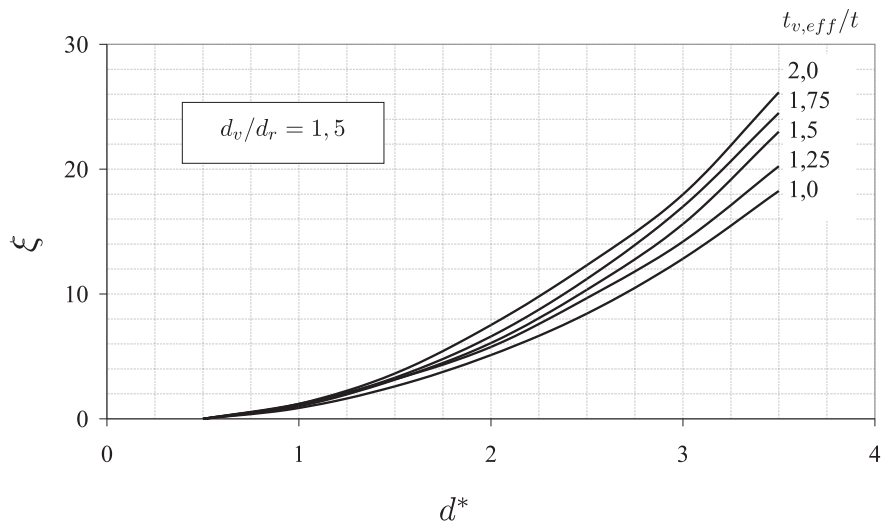

Abbildung 7.48: $\quad \xi$ zur Bestimmung des kritischen Momentes $M_{z, \text { crit,GNA,ver }}^{*}$ von Kreiszylinderschalen mit Verstärkungsblechen mit $1,0 \leq t_{v, \text { eff }} / t \leq 2,0$ für $d_{v} / d_{r}=1,5$

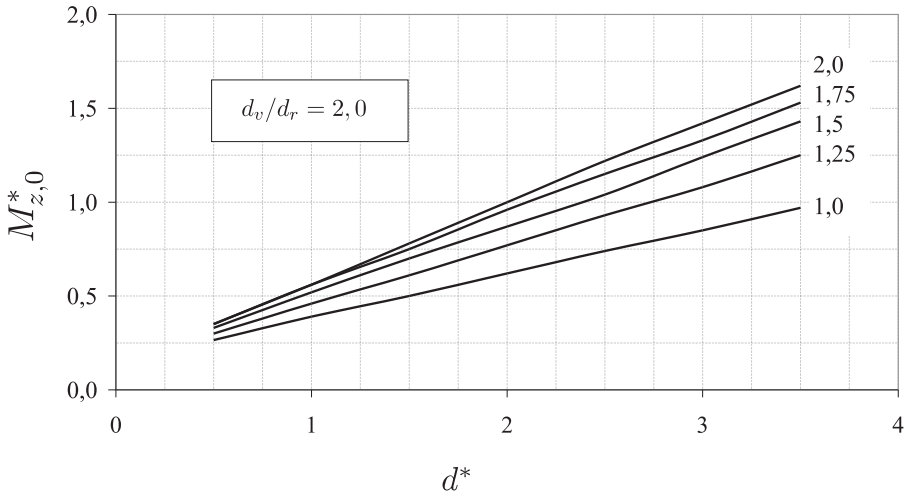

Abbildung 7.49: $\quad M_{z, 0}^{*} \quad$ zur Bestimmung des kritischen Momentes $M_{z, \text { crit }, G N A, \text { ver }}^{*}$ von Kreiszylinderschalen mit Verstärkungsblechen mit $1,0 \leq t_{v, \text { eff }} / t \leq 2,0$ für $d_{v} / d_{r}=2,0$ 


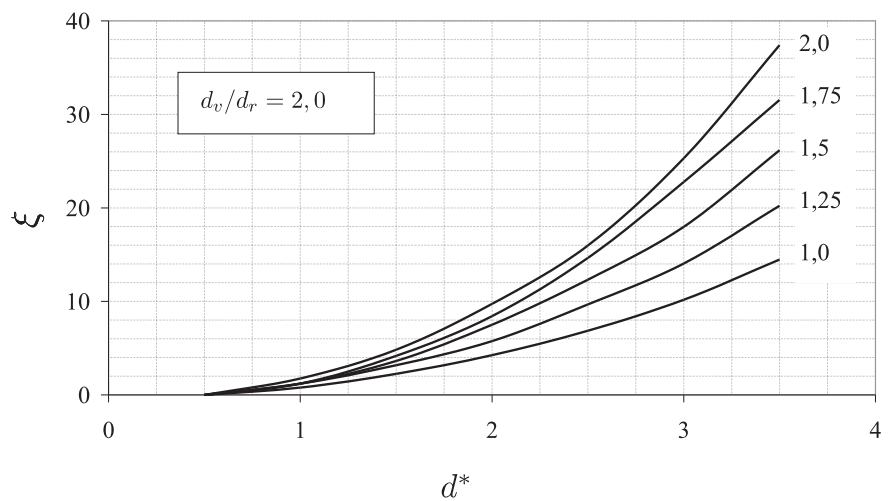

Abbildung 7.50: $\quad \xi \quad$ zur Bestimmung des kritischen Momentes $M_{z, \text { crit }, \text { GNA,ver }}^{*}$ von Kreiszylinderschalen mit Verstärkungsblechen mit $1,0 \leq t_{v, \text { eff }} / t \leq 2,0$ für $d_{v} / d_{r}=2,0$

Die Anfangssteifigkeit $c_{M_{z, v e r}}^{*}$ der untersuchten Kreiszylinderschalen kann analog dazu mit

$$
c_{0, M_{z}, v e r}^{*}=c_{0, M_{z}}^{*}+\frac{\omega}{L^{*}}
$$

bestimmt werden. Die Werte $c_{0, M_{z}}^{*}$ können aus Abbildung 7.51 und $\omega$ aus Abbildung 7.52 für Kreiszylinderschalen mit $d_{v} / d_{r}=1,5$ abgelesen werden. Für Kreiszylinderschalen mit $d_{v} / d_{r}=2,0$ kann $c_{0, M_{z}}^{*}$ aus Abbildung 7.53 und $\omega$ aus Abbildung 7.54 abgelesen werden. Für $d_{v} / d_{r}$-Verhältnisse, die zwischen diesen Werte liegen, kann bei der Ermittlung von $c_{M_{z, v e r}}^{*}$ linear interpoliert werden. 


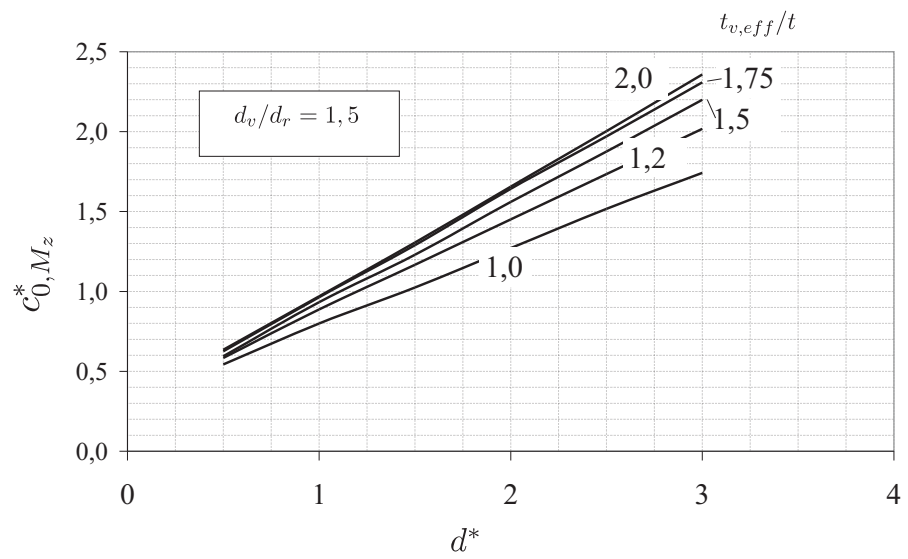

Abbildung 7.51: $c_{0, M_{z}}^{*}$ zur Bestimmung der Anfangssteifigkeit $c_{M_{z, v e r}}^{*}$ von Kreiszylinderschalen mit Verstärkungsblechen mit $1,0 \leq t_{v, \text { eff }} / t \leq 2,0$ für $d_{v} / d_{r}=1,5$

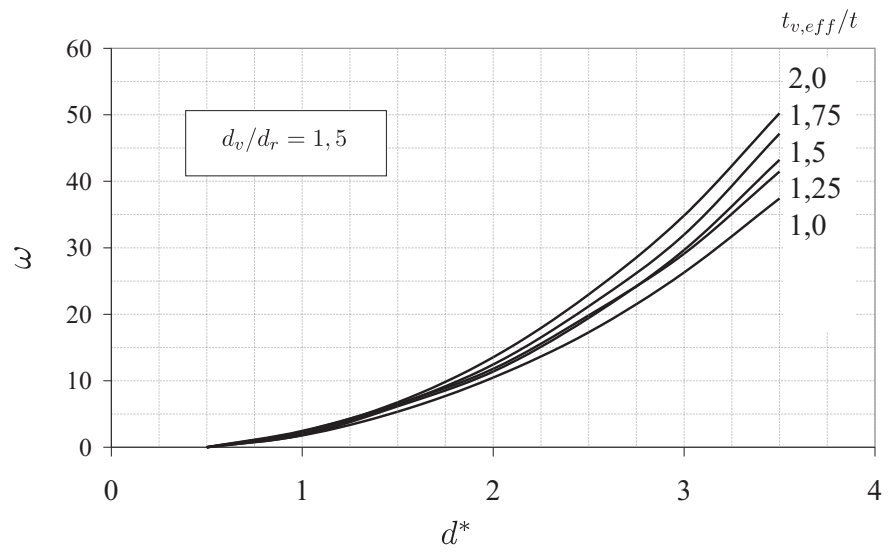

Abbildung 7.52: $\omega$ zur Bestimmung der Anfangssteifigkeit $c_{M_{z, v e r}}^{*}$ von Kreiszylinderschalen mit Verstärkungsblechen mit $1,0 \leq t_{v, \text { eff }} / t \leq 2,0$ für $d_{v} / d_{r}=1,5$ 


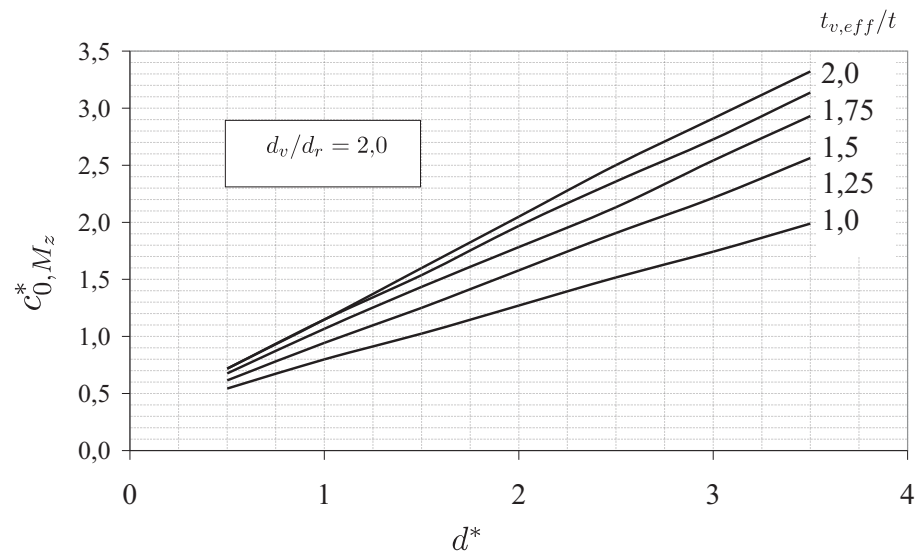

Abbildung 7.53: $c_{0, M_{z}}^{*}$ zur Bestimmung der Anfangssteifigkeit $c_{M_{z, v e r}}^{*}$ von Kreiszylinderschalen mit Verstärkungsblechen mit $1,0 \leq t_{v, \text { eff }} / t \leq 2,0$ für $d_{v} / d_{r}=2,0$

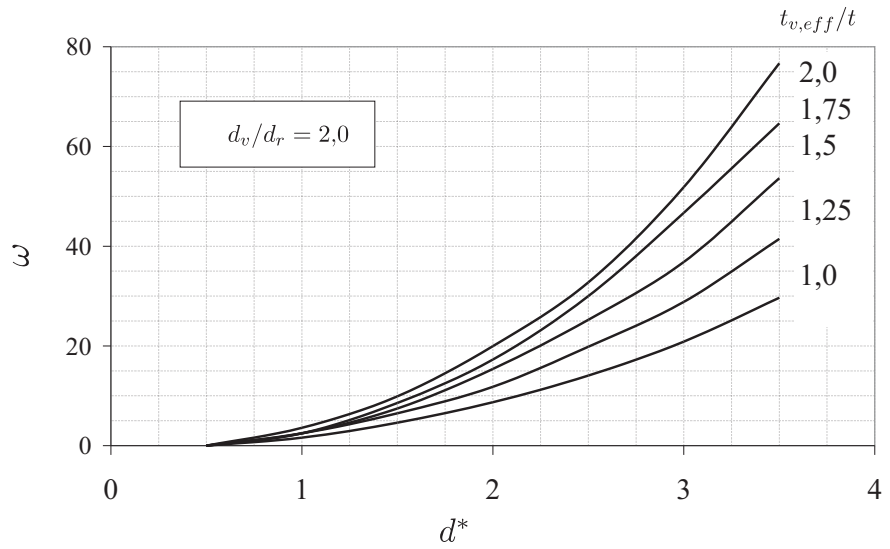

Abbildung 7.54: $\omega$ zur Bestimmung der Anfangssteifigkeit $c_{M_{z, v e r}}^{*}$ von Kreiszylinderschalen mit Verstärkungsblechen mit $1,0 \leq t_{v, \text { eff }} / t \leq 2,0$ für $d_{v} / d_{r}=2,0$ 


\subsubsection{Der Einfluss materieller Nichtlinearität auf das Tragver- halten verstärkter Kreiszylinderschalen}

Wie Abbildung 7.55 am Beispiel einer Kreiszylinderschale mit $L^{*}=20$, $R / t=400$ und $d^{*}=3,0$ mit einem Verstärkungsblech mit $d_{v} / d_{r}=2,0$ für unterschiedliche Blechdickenverhältnisse $t_{v, \text { eff }} / t$ und unterschiedliche Streckgrenzen $f_{y}$ zeigt, macht sich der Einfluss des plastischen Werkstoffverhaltens auf das Tragverhalten erst im Bereich sehr großer Verformungen bemerkbar. Eine Abminderung des kritischen Momentes $M_{z, \text { crit }, G M N A, v e r}^{*}$ ist daher nicht erforderlich.

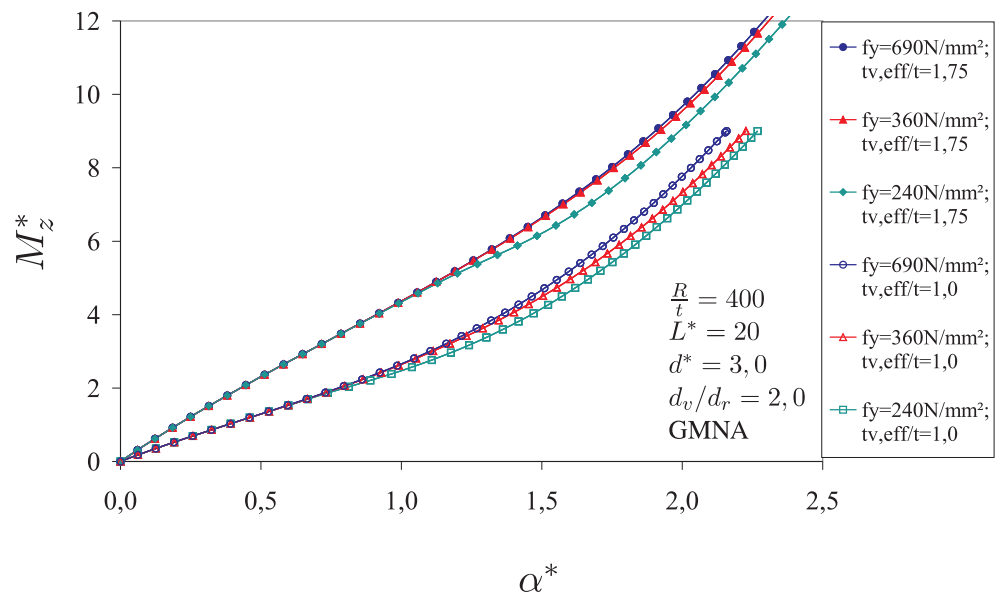

Abbildung 7.55: Momenten-Rotationskurven einer Kreiszylinderschale mit $L^{*}=20, R / t=400$ und $d^{*}=3,0$ mit einem Verstärkungsblech mit $d_{v} / d_{r}=2,0$ für unterschiedliche $t_{v, \text { eff }} / t$ und $f_{y}$

\subsection{Zusammenfassung der Ergebnisse}

Wie die Untersuchungen an scheibenförmig verstärkten Kreiszylinderschalen gezeigt haben, wird das Tragverhalten durch die Verstärkung erheblich beeinflusst. Unter Berücksichtigung der effektiven Blechdicke $t_{v, \text { eff }}$ ist es allerdings unerheblich, ob die Bleche in das Mantelblech eingesetzt oder auf das Mantelblech aufgesetzt werden. In Abhängigkeit vom Durchmesserverhältnis $d_{v} / d_{r}$ und Blechdickenverhältnis $t_{v, \text { eff }} / t$ der Verstärkungsbleche können die 
Spannungen am Stutzenanschluss um bis zu 70\% reduziert werden. Die Steifigkeit der Kreiszylinderschalen nimmt durch die Verstärkungsbleche um bis zu 250\% zu. Bei Stabilitätsgefahr können die kritischen Lasten durch die Verstärkungsbleche um bis zu 300\% gesteigert werden. Der Einfluss des plastischen Werkstoffverhaltens kann vereinfachend und auf der sicheren Seite liegen durch die Abminderungskurven der unverstärkten Kreiszylinderschalen berücksichtigt werden. 


\section{Anwendungsbeispiele}

\subsection{Tank mit einem Durchmesser von 8 m unter Einwirkung von $P_{x}$ und $M_{\varphi}$}

Die Anwendung der in dieser Arbeit erstellten Bemessungsvorlage wird am Beispiel eines Tanks betrachtet. Dazu wird der in Abbildung 8.1 dargestellte Tank mit einem Radius $R$ von $4 \mathrm{~m}$, einer konstanten Wanddicke $t$ von $5 \mathrm{~mm}$ und einer Höhe $H$ von $16 \mathrm{~m}$ betrachtet. Auf einer Höhe von $8 \mathrm{~m}$ ist ein Stutzen mit einem Durchmesser $d_{r}$ von $400 \mathrm{~mm}$ und einer Wanddicke von $5 \mathrm{~mm}$ an den Tank angeschlossen. Der Abstand $L$ des Stutzens zum Schalenrand beträgt daher jeweils $8 \mathrm{~m}$. Am unteren Rand ist der Tank auf ein Bodenblech geschweißt. Am oberen Rand schließt der Tank durch ein festes Dach ab. Für Tank und Stutzen wird ein Baustahl S235 mit einer Streckgrenze $f_{y}$ von $240 \mathrm{~N} / \mathrm{mm}^{2}$ und einem E-Modul von $210000 \mathrm{~N} / \mathrm{mm}^{2}$ verwendet. Der Stutzen wird durch eine radiale Einzellast $P_{x}$ von $3 \mathrm{kN}$ und ein Moment $M_{\varphi}$ in Umfangsrichtung von $1 \mathrm{kNm}$ beansprucht. Imperfektionen spielen eine untergeornete Rolle und werden nicht berücksichtigt. Die Abmessungen und die bezogenen Größen sind in Tabelle 8.1 aufgeführt.

\begin{tabular}{|c|c|c|}
\hline \multicolumn{3}{|c|}{ Abmessungen } \\
\hline Radius & $R$ & $4000 \mathrm{~mm}$ \\
\hline Wanddicke & $t$ & $5 \mathrm{~mm}$ \\
\hline Stutzendurchmesser & $d_{r}$ & $400 \mathrm{~mm}$ \\
\hline Wanddicke Stutzen & $t_{r}$ & $5 \mathrm{~mm}$ \\
\hline Randabstand & $\bar{L}$ & $8000 \mathrm{~mm}$ \\
\hline Streckgrenze & $f_{y}$ & $240 \mathrm{~N} / \mathrm{mm}^{2}$ \\
\hline E-Modul & $E$ & $210000 \mathrm{~N} / \mathrm{mm}^{2}$ \\
\hline \multicolumn{3}{|c|}{ bezogene Größen } \\
\hline$R / t$-Verhältnis & $R / t$ & 800 \\
\hline bezogener Stutzendurchmesser & $d^{*}$ & 2,83 \\
\hline bezogener Randabstand & $L^{*}$ & 56,6 \\
\hline Plastizitätsfaktor & $\eta$ & 0,914 \\
\hline
\end{tabular}

Tabelle 8.1: Abmessungen Tank 1 


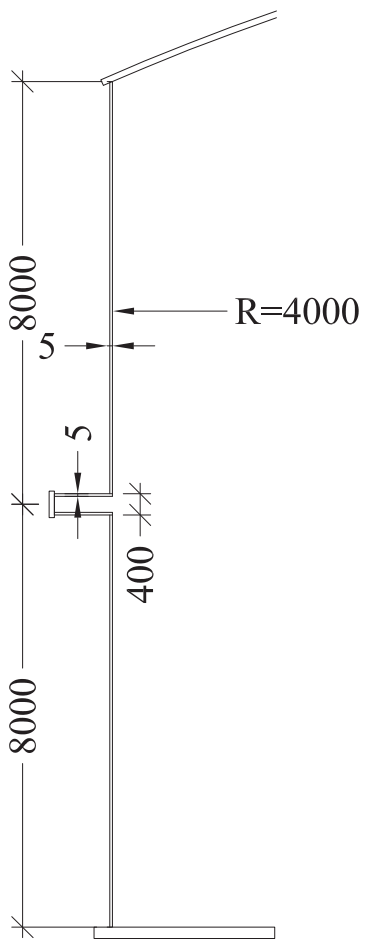

Abbildung 8.1: Schnitt durch Tank 1

\section{Lastfall $P_{x}$}

Die bezogene kritische Last $P_{x, c r i t, G N A}^{*}$ unter Berücksichtigung der geometrischen Nichtlinearität ergibt sich aus Gleichung 7.9 mit

$$
\begin{array}{ll}
d^{*}=2,83 & \\
L^{*}=56,6 & \\
P_{0}^{*}=1,2 & \text { aus Abbildung } 7.15 \\
\psi=7 & \text { aus Abbildung } 7.16 \mathrm{zu} \\
P_{x, \text { crit }, G N A}^{*}=P_{0}^{*}+\frac{\psi}{L^{*}}=1,2+\frac{7}{56,6}=1,32
\end{array}
$$

Dies entspricht einer tatsächlichen kritischen Last von

$$
P_{x, c r i t, G N A}=\frac{P^{*} E t^{3}}{R}=\frac{1,32 \cdot 210000 \mathrm{~N} / \mathrm{mm}^{2} \cdot(5 \mathrm{~mm})^{3}}{4000}=8,66 \mathrm{kN}
$$

Alternativ dazu kann $P_{x, c r i t, G N A}^{*}$ auch aus Abbildung 6.11 abgelesen werden. 
Die Abminderung der bezogenen kritischen Last $P_{x, c r i t, G N A}^{*}$ infolge des plastischen Werkstoffverhaltens ergibt sich aus Gleichung 6.9 und $\eta=0,914 \mathrm{zu}$

$$
\begin{aligned}
P_{x, c r i t, G M N A}^{*} / P_{x, c r i t, G N A}^{*} & =-0,245 \eta^{2}+0,66 \eta+0,56 \\
& =-0,245 \cdot 0,914^{2}+0,66 \cdot 0,914+0,56=0,96
\end{aligned}
$$

Die bezogene kritische Last $P_{x, c r i t, G M N A}^{*}$ beträgt folglich

$$
P_{x, \text { crit }, G M N A}^{*}=0,96 \cdot 1,32=1,27
$$

Dies entspricht einer tatsächlichen kritischen Last von

$$
P_{x, c r i t, G M N A}=\frac{P^{*} E t^{3}}{R}=\frac{1,27 \cdot 210000 \mathrm{~N} / \mathrm{mm}^{2} \cdot(5 \mathrm{~mm})^{3}}{4000}=\mathbf{8 , 3 3} \mathbf{~ k N}
$$

Aus Abschnitt 6.4.6 folgt, dass bis zu einer bezogenen Last von

$$
0,4 \cdot P_{x, \text { crit }, G N A}^{*}=0,4 \cdot 1,32=0,53
$$

die Last-Verformungsbeziehung des Tanks linear ist. Dies entspricht einer tatsächlichen Last $P_{x}$ von

$$
P_{x}=\frac{P^{*} E t^{3}}{R}=\frac{0,53 \cdot 210000 \mathrm{~N} / \mathrm{mm}^{2} \cdot(5 \mathrm{~mm})^{3}}{4000}=3,48 \mathrm{kN}
$$

Die radiale Einzellast $P_{x}=3 \mathrm{kN}$ ist demnach kleiner als die linear elastische Grenzlast von $3,48 \mathrm{kN}$. Die maximalen bezogenen Spannungen $\sigma_{\varphi, \max , P_{x}}^{*} \mathrm{im}$ Stutzenanschlussbereich werden mit Gleichung 6.6 und

$$
\begin{aligned}
d^{*}=2,83 & \\
\sigma_{\varphi, \max , P_{x}}^{*} & =-0,5 d^{*}+2,3 \sqrt{d^{*}}-3,2 \\
& =-0,5 \cdot 2,83+2,3 \sqrt{2,83}-3,2=-0,746
\end{aligned}
$$

ermittelt. Unter Einwirkung einer radialen Einzellast $P_{x}$ von $3 \mathrm{kN}$ ergibt sich daraus eine tatsächliche maximale Spannung $\sigma_{\varphi, \max , P_{x}}$ von

$$
\begin{aligned}
\sigma_{\varphi, \max , P_{x}} & =\sigma_{\varphi, \max , P_{x}}^{*} \frac{P_{x}}{t^{2}} \\
& =-0,746 \frac{3000 N}{(5 m m)^{2}}=\mathbf{- 9 0} \mathbf{~ N} / \mathbf{m m}^{2}
\end{aligned}
$$

Der Betrag der Druckspannung kann näherungsweise und auf der sicheren Seite liegend dem Betrag der Zugspannungen gleichgesetzt werden.

Die Anfangssteifigkeit $c_{P_{x}}^{*}$ ergibt sich aus Gleichung $7.10 \mathrm{mit}$

$$
\begin{aligned}
& d^{*}=2,83 \\
& L^{*}=56,6 \\
& c_{0, P_{x}}^{*}=8 \quad \text { aus Abbildung } 7.19 \\
& \chi=-0,77 \quad \text { aus Abbildung } 7.20 \mathrm{zu} \\
& c_{P_{x}}^{*}=c_{0, P_{x}}^{*} \cdot\left(L^{*}\right)^{\chi}=8 \cdot(56,6)^{-0,77}=0,36
\end{aligned}
$$


$c_{P_{x}}^{*}$ kann für eine unverstärkte Kreiszylinderschale auch aus Abbildung 6.12 abgelesen werden.

Die maximale radiale Verschiebung $u_{x}$ unter einer Last $P_{x}$ von $3 \mathrm{kN}$ ergibt sich durch Umformung der Anfangssteifigkeit

$$
\begin{aligned}
c_{P_{x}}^{*} & =\frac{P_{x}^{*}}{u^{*}}=\frac{P_{x}}{u_{x}} \cdot \frac{R}{E t^{2}} \mathrm{zu} \\
u_{x} & =\frac{P_{x}}{c_{P x}^{*}} \frac{R}{E t^{2}} \\
& =\frac{3000 \mathrm{kN}}{0,36} \cdot \frac{4000 \mathrm{~mm}}{210000 \mathrm{~N} / \mathrm{mm}^{2} \cdot(5 \mathrm{~mm})^{2}}=\mathbf{6 , 3 5} \mathbf{~ m m}
\end{aligned}
$$

Die Ergebnisse für den Lastfall $P_{x}$ sind in Tabelle 8.2 zusammengefasst. Dazu sind auch die Ergebnisse aus einer FE-Berechnung aufgeführt. Diese stimmen gut mit den Werten aus den Bemessungstabellen überein.

\begin{tabular}{|l|l|l|}
\hline & Bemessungsdiagramme & FEM \\
\hline$P_{x, \text { crit }, \text { GNA }}$ & $8,66 \mathrm{kN}$ & $8,60 \mathrm{kN}$ \\
\hline$P_{x, \text { crit }, \text { GMNA }}$ & $8,33 \mathrm{kN}$ & $8,37 \mathrm{kN}$ \\
\hline$c_{P_{x}}^{*}$ & 0,36 & 0,38 \\
\hline \multicolumn{2}{|c|}{$P_{x}=3 \mathrm{kN}$} \\
\hline$\sigma_{\varphi, \max , P_{x}}$ & $-90 \mathrm{~N} / \mathrm{mm}^{2}$ & $-90 \mathrm{~N} / \mathrm{mm}^{2}$ \\
\hline$u_{x}$ & $6,35 \mathrm{~mm}$ & $6,00 \mathrm{~mm}$ \\
\hline
\end{tabular}

Tabelle 8.2: Vergleich der mittels Bemessungsdiagrammen ermittelten Ergebnisse mit Ergebnissen aus FE-Berechnungen für den Lastfall $P_{x}$

\section{Lastfall $M_{\varphi}$}

Das bezogene kritische Moment $M_{\varphi, c r i t, G N A}^{*}$ unter Berücksichtigung der geometrischen Nichtlinearität kann aus Abbildung 7.33 abgelesen werden:

$$
M_{\varphi, c r i t, G N A}^{*}=4,3
$$

Das entsprechende tatsächlich Moment ergibt sich aus

$$
\begin{aligned}
M_{\varphi, \text { crit }, G N A} & =M^{*} \cdot E t^{3} \cdot \sqrt{\frac{t}{R}} \\
& =4,3 \cdot 210000 \mathrm{~N} / \mathrm{mm}^{2} \cdot(5 \mathrm{~mm})^{3} \cdot \frac{5}{4000}=\mathbf{4 , 0 0} \mathbf{~} \mathbf{N m}
\end{aligned}
$$

Die Abminderung des bezogenen kritischen Moments $M_{\varphi, c r i t, G N A}^{*}$ infolge des plastischen Werkstoffverhaltens ergibt sich aus Gleichung 6.19 und $\eta=0,914 \mathrm{zu}$

$$
\begin{aligned}
M_{\varphi, c r i t, G M N A}^{*} / M_{\varphi, \text { crit }, \text { GNA }}^{*} & =-0,56 \eta^{2}+1,45 \eta+0,07 \\
& =-0,56 \cdot 0,914^{2}+1,45 \cdot 0,914+0,07=0,93
\end{aligned}
$$

Das kritische Moment $M_{\varphi, \text { crit }, \text { GMNA }}$ beträgt folglich

$$
M_{\varphi, \text { crit }, G M N A}=0,93 \cdot 4,0 \mathrm{kNm}=\mathbf{3 , 7 2} \mathrm{kNm}
$$


Aus Abschnitt 6.5.6 folgt, dass die Kreiszylinderschale sich bis zu einem Moment von

$0,4 \cdot M_{\varphi, c r i t, G N A}=0,4 \cdot 4,0 \mathrm{kNm}=1,6 \mathrm{kNm}$

linear elastisch verhält.

Bei alleiniger Belastung des Tanks durch ein Moment $M_{\varphi}$ in Umfangsrichtung von $1 \mathrm{kNm}$ ergibt sich mit Gleichung 6.15 die maximale bezogene Spannung im Stutzenanschlussbereich von

$$
\begin{aligned}
\sigma_{z, \max , M_{\varphi}}^{*} & =0,69 \sqrt{d^{*}}-2,1 \\
& =0,69 \cdot \sqrt{2,83}-2,1=-0,94
\end{aligned}
$$

Dies entspricht einer tatsächlichen maximalen Spannung $\sigma_{z, \max , M_{\varphi}}$ von

$$
\begin{aligned}
\sigma_{z, \max , M_{\varphi}} & =\sigma_{z, \max , M_{\varphi}} \frac{M_{\varphi}}{d_{r} \cdot t^{2}} \\
& =-0,94 \frac{1 \mathrm{kNm}}{400 \mathrm{~mm} \cdot(5 \mathrm{~mm})^{2}}=\mathbf{- 9 4} \mathbf{~ N} / \mathbf{m m}^{2}
\end{aligned}
$$

Der Betrag der Druckspannung kann näherungsweise und auf der sicheren Seite liegend dem Betrag der Zugspannungen gleichgesetzt werden.

Die Anfangssteifigkeit $c_{M_{\varphi}}^{*}$ beträgt für diesen Lastfall gemäß Abbildung 7.35

$$
c_{M_{\varphi}}^{*}=8
$$

Durch Umformung von

$$
c_{M_{\varphi}}^{*}=\frac{M_{\varphi}^{*}}{\alpha^{*}}=\frac{M_{\varphi}}{\alpha} \cdot \frac{1}{E t^{3}}
$$

ergibt sich eine maximale Verdrehung $\alpha$ des Stutzens von

$$
\begin{aligned}
\alpha & =\frac{M \varphi}{c_{M \varphi}^{*}} \cdot \frac{1}{E t^{3}} \\
& =\frac{100 \mathrm{kNcm}}{8} \cdot \frac{1}{210000 \mathrm{~N} / \mathrm{mm}^{2} \cdot(5 \mathrm{~mm})^{3}} \\
& =0,005 \mathrm{rad}
\end{aligned}
$$

Diese Verdrehung entspricht einer maximalen radialen Verschiebung $u_{x}$ der Tankwand am Stutzenanschluss von

$$
u_{x}=\alpha \cdot \frac{d_{r}}{2}=\mathbf{1} \mathbf{~ m m}
$$

Die Ergebnisse für den Lastfall $M_{\varphi}$ sind in Tabelle 8.3 aufgeführt. Dort sind auch die Ergebnisse aus einer FE-Berechnung angegeben. Diese stimmen sehr gut mit den Werten aus den Bemessungstabellen überein.

\section{Überlagerung von $P_{x}$ und $M_{\varphi}$}

In den Abschnitten 6.4.2 und 6.5.2 wurden vereinfachend nur die maximalen Spannungen betrachtet. Für den Lastfall $P_{x}$ sind dies die Umfangsspannungen $\sigma_{\varphi, \max , P_{x}}$ und für den Lastfall $M_{\varphi}$ die Längsspannungen $\sigma_{z, \max , M_{\varphi}}$, die mit den Gleichungen 6.6 und 6.15 bestimmt werden können. Bei gleichzeitiger Einwirkung von $P_{x}$ und $M_{\varphi}$ kann bei der Spannungsüberlagerung als grobe Näherung der Betrag der Umfangsspannungen dem der Längsspannungen gleichgesetzt 


\begin{tabular}{|l|l|l|}
\hline & Bemessungsdiagramme & FEM \\
\hline$M_{\varphi, G N A}$ & $4,0 \mathrm{kNm}$ & $4,66 \mathrm{kNm}$ \\
\hline$M_{\varphi, G M N A}$ & $3,7 \mathrm{kNm}$ & $4,43 \mathrm{kNm}$ \\
\hline$c_{M_{\varphi}}^{*}$ & 8,0 \\
\hline \multicolumn{3}{|c|}{$M_{\varphi}=1 \mathrm{kNm}$} \\
\hline \multicolumn{3}{|c|}{8,47} \\
\hline$\sigma_{z, \max , M_{\varphi}}$ & Bemessungsdiagramme & FEM \\
\hline$\alpha$ & $0,-94 \mathrm{~N} / \mathrm{mm}^{2}$ & $-83 \mathrm{~N} / \mathrm{mm}^{2}$ \\
\hline
\end{tabular}

Tabelle 8.3: Vergleich der mittels Bemessungsdiagrammen ermittelten Ergebnisse mit Ergebnissen aus FE-Berechnungen für den Lastfall $M_{\varphi}$

werden.

Die überlagerte maximale Spannung $\sigma_{\max }$ am Stutzenanschluss beträgt folglich

$$
\begin{aligned}
\sigma_{\max } & =\sigma_{\varphi, \max , P_{x}}+\sigma_{z, \max , M_{\varphi}} \\
& =-90 \mathrm{~N} / \mathrm{mm}^{2}-94 \mathrm{~N} / \mathrm{mm}^{2}=\mathbf{- 1 8 4} \mathbf{N} / \mathbf{m m}^{2}
\end{aligned}
$$

Bei Überlagerung der radialen Verschiebungen im Stutzenanschlussbereich ergibt sich eine maximale radiale Verschiebung

$$
u_{x, \max }=u_{x, P_{x}}+u_{x, M_{\varphi}}=7,39 \mathrm{~mm}+1 \mathrm{~mm}=\mathbf{7 , 3 9}
$$

Aufgrund der oben getroffenen Vereinfachungen liegt die überlagerte Maximalspannung $\sigma_{\max }$ weit über der maximalen Spannung aus einer FE-Berechnung, wie der Vergleich der Ergebnisse in Tabelle 8.4 zeigt. Die maximale radiale Verschiebung $u_{x, \max }$ stimmt dagegen sehr gut mit dem numerisch ermittelten Ergebnis überein.

\begin{tabular}{|l|l|l|}
\hline \multicolumn{3}{|c|}{$P_{x}=3 \mathrm{kN}$ und $M_{\varphi}=1 \mathrm{kNm}$} \\
\hline \hline & Bemessungsdiagramme & FEM \\
\hline$\sigma_{\max }$ & $-184 \mathrm{~N} / \mathrm{mm}^{2}$ & $-111 \mathrm{~N} / \mathrm{mm}^{2}$ \\
\hline$u_{x, \max }$ & $7,39 \mathrm{~mm}$ & $7,2 \mathrm{~mm}$ \\
\hline
\end{tabular}

Tabelle 8.4: Vergleich der mittels Bemessungsdiagrammen ermittelten Ergebnisse mit Ergebnissen aus FE-Berechnungen bei gleichzeitiger Einwirkung von $P_{x}$ und $M_{\varphi}$ 


\subsection{Tank mit einem Durchmesser von 20 m unter Einwirkung von $P_{x}$}

Im zweiten Anwendungsbeispiel wird ein Tank mit einem Radius von $20 \mathrm{~m}$, einer Höhe von $16 \mathrm{~m}$ und Mantelblechdicken zwischen $10 \mathrm{~mm}$ und $16 \mathrm{~mm}$ betrachtet. Am unteren Schuss ist ein Stutzen mit einem Außendurchmesser von $610 \mathrm{~mm}$ und einer Wanddicke von $16 \mathrm{~mm}$ angeschlossen. Der unterste Schuss hat eine Blechdicke von $16 \mathrm{~mm}$. Der Abstand des Stutzens zum Bodenblech beträgt $880 \mathrm{~mm}$. Der Tank wird durch ein sechseckiges, auf das Mantelblech aufgesetztes Verstärkungsblech mit einer Blechdicke $t_{v}$ von $20 \mathrm{~mm}$ verstärkt. In Abbildung 8.2 (a) ist ein Schnitt durch den Tank dargestellt. In Abbildung 8.2 (b) ist eine detaillierte Darstellung des Stutzens abgebildet. Aufgrund der Stutzenanordnung sind abweichend vom Berechnungsmodell nach Abbildung 7.1 bei diesem Tank die Symmetriebedingungen nicht eingehalten. Das ausgeführte Verstärkungsblech entspricht nicht dem kreisrunden Blech des Berechungsmodells. Das Verstärkungsblech wird daher mit einem in das Sechseck einbeschriebenen Kreisquerschnitt mit einem Duchmesser $d_{v}$ von 1000 mm gedanklich ersetzt. Der Werkstoff des Tanks, des Stutzens und des Verstärkungsbleches ist ein S355 mit einer Streckgrenze von $360 \mathrm{~N} / \mathrm{mm}^{2}$ und einem E-Modul von $210000 \mathrm{~N} / \mathrm{mm}^{2}$. Der Stutzen wird durch eine radiale Einzellast $P_{x}=10 \mathrm{kN}$ belastet. Aus den Abmessungen ergeben sich die in Tabelle 8.5 aufgeführten bezogenen Größen.

die efektive Blechdicke $t_{v, \text { eff }} / t$ ergibt sich aus Gleichung $7.2 \mathrm{zu}$

$$
t_{v, \text { eff }}=t \sqrt[3]{\left(\frac{t_{v}}{t}\right)^{3}+1}=16 \mathrm{~mm} \cdot \sqrt[3]{\left(\frac{16 m m}{16 m m}\right)^{3}+1}=20,16 \mathrm{~mm}
$$

Mit diesem Blechdickenverhältnis $t_{v, \text { eff }} / t=1,26$ kann bei einem bezogenen Stutzendurchmesser $d^{*}$ von 1,08 aus Abbildung 7.9 ein Mindestdurchmesserverhältnis

$$
\left(d_{v} / d_{r}\right)_{\min }=1,5
$$

abgelesen werden. Da

$$
d_{v} / d_{r}=(1,64)_{v o r h}>\left(d_{v} / d_{r}\right)_{\min }=1,5
$$

ist die Forderung erfüllt, dass die Spannungen im unverstärkten Mantelblech geringer sind als im Verstärkungsblech.

Die Abminderung der Spannung infolge des Verstärkungsbleches ergibt sich aus Gleichung $7.6 \mathrm{zu}$ 
(a)

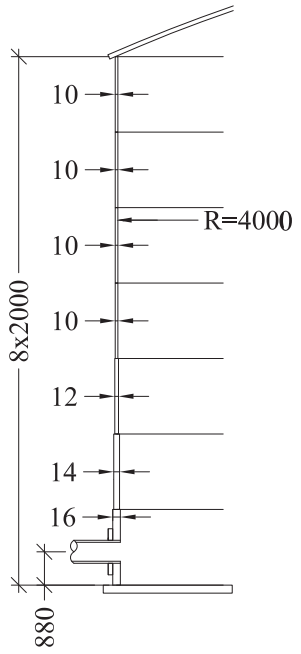

(b)

Ersatzkreisquerschnitt

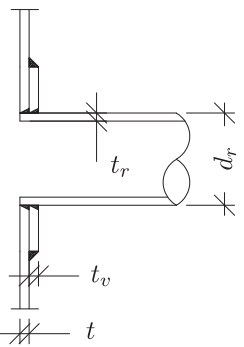

Abbildung 8.2: Schnitt durch Tank 2

\begin{tabular}{|l|l|l|}
\hline \multicolumn{3}{|c|}{ Abmessungen } \\
\hline \hline Radius & $R$ & $20000 \mathrm{~mm}$ \\
\hline Wanddicke & $t$ & $10 \div 16 \mathrm{~mm}$ \\
\hline Stutzendurchmesser & $d_{r}$ & $610 \mathrm{~mm}$ \\
\hline Wanddicke Stutzen & $t_{r}$ & $16 \mathrm{~mm}$ \\
\hline Randabstand & $L$ & $880 \mathrm{~mm}$ \\
\hline Verstärkungsblechdurchmesser & $d_{v}$ & $1000 \mathrm{~mm}$ \\
\hline Verstärkungsblechdicke & $t_{v}$ & $16 \mathrm{~mm}$ \\
\hline Streckgrenze & $f_{y}$ & $360 \mathrm{~N} / \mathrm{mm}^{2}$ \\
\hline E-Modul & $E$ & $210000 \mathrm{~N} / \mathrm{mm}^{2}$ \\
\hline \hline
\end{tabular}

bezogene Größen

\begin{tabular}{|l|l|l|}
\hline$R / t$-Verhältnis & $R / t$ & 1250 \\
\hline bezogener Stutzendurchmesser & $d^{*}$ & 1,08 \\
\hline bezogener Randabstand & $L^{*}$ & 1,56 \\
\hline Durchmesserverhältnis & $d_{v} / d_{r}$ & 1,64 \\
\hline Plastizitätsfaktor & $\eta$ & 2,14 \\
\hline
\end{tabular}

Tabelle 8.5: Abmessungsen Tank 2 


$$
\begin{aligned}
\frac{\sigma_{v e r, P_{x}}^{*}}{\sigma_{\varphi, \max , P_{x}}^{*}} & =1,16\left(\frac{t}{t_{v, \text { eff }}}\right)-0,16 \\
& =1,16 \cdot \frac{16 \mathrm{~mm}}{20,16 \mathrm{~mm}}-0,16=0,76
\end{aligned}
$$

Nach Gleichung 6.6 beträgt beträgt die maximale bezogene Spannung $\sigma_{\varphi, \max , P_{x}}^{*}$ am Stutzenanschluss eines unverstärkten Tanks

$$
\begin{aligned}
\sigma_{\varphi, \max , P_{x}}^{*} & =-0,5 d^{*}+2,3 \sqrt{d^{*}}-3,2 \\
& =-0,5 \cdot 1,08+2,3 \sqrt{1,08}-3,2=-1,35
\end{aligned}
$$

Unter Einwirkung einer radialen Einzellast $P_{x}$ von $10 \mathrm{kN}$ entspricht dies einer tatsächlichen maximalen Spannung $\sigma_{P_{x}, \max }$ am Stutzenanschluss eines unverstärkten Tanks von

$$
\begin{aligned}
\sigma_{\varphi, \max , P_{x}} & =\sigma_{\varphi, \max , P_{x}}^{*} \frac{P_{x}}{t^{2}} \\
& =-1,35 \frac{10000 N}{16 m m^{2}}=\mathbf{- 5 3} \mathbf{N} / \mathbf{m m}^{2}
\end{aligned}
$$

Die abgeminderte Spannung $\sigma_{v e r, P_{x}}$ im Verstärkungsblech beträgt folglich

$$
\sigma_{\text {ver }, P_{x}}=0,76 \cdot\left(-53 \mathrm{~N} / \mathrm{mm}^{2}\right)=\mathbf{- 4 0} \mathbf{N} / \mathbf{m m}^{2}
$$

Bei der Ermittlung der kritischen bezogenen Last $P_{x, c r i t, G N A}^{*}$ unter Berücksichtigung der geometrischen Nichtlinearität wird auf der sicheren Seite liegend mit einem Durchmesserverhältnis $d_{v} / d_{r}$ von 1,5 gerechnet. Da der randnahe Stutzen mit einem bezogenen Randabstand $L^{*}$ von 1,56 zu weitaus größeren kritischen Lasten führt, als mit der in den Bemessungsdiagrammen angegebene untere Grenze von $L^{*}=10$, wird auf der sicheren Seite liegend mit $L^{*}=10$ gerechnet. Mit einem Blechdickenverhältnis $t_{v, \text { eff }} / t$ von 1,26 und einem bezogenen Stutzendurchmesser $d^{*}=1,08$ wird $P_{x, c r i t, G N A}^{*}$ mit Gleichung 7.9 ermittelt. Mit

$$
\begin{aligned}
& L^{*}=10 \\
& P_{0}^{*}=0,9 \quad \text { aus Abbildung } 7.15 \\
& \psi=0,2 \quad \text { aus Abbildung } 7.16 \text { folgt } \\
& P_{x, \text { crit }, \text { GNA }}^{*}=P_{0}^{*}+\frac{\psi}{L^{*}}=0,9+\frac{0,2}{10}=0,92
\end{aligned}
$$

Da der Plastizitätsfaktor $\eta=2,14$ größer als 1,2 ist, hat das plastische Werkstoffverhalten keinen Einfluss auf das Tragverhalten des Tanks. 


\section{Zusammenfassung}

In der vorliegenden Arbeit wird das Tragverhalten von Schalentragwerken unter Einwirkung von radialen Einzellasten und Momenten untersucht. Der Schwerpunkt der Arbeit liegt dabei auf der numerischen Untersuchung lokal beanspruchter Kreiszylinderschalen wobei auch kreisförmige Verstärkungsmaßnahmen im Lasteinleitungsbereich der Kreiszylinderschale berücksichtigt werden.

Der in dieser Arbeit zusammengetragene Wissenstand zum LastVerformungsverhalten lokal beanspruchter Kreiszylinderschalen geht aus zahlreichen experimentellen und theoretischen Untersuchungen der vergangenen 70 Jahre hervor. Die theoretischen Arbeiten basieren dabei auf der Annahme, dass nur kleine Verformungen auftreten, was zur Folge hat, dass der Anwendungsbereich der Bemessungsregeln auf Kreiszylinderschalen mit $R / t<1250$ beschränkt ist. Da das Tragverhalten von Kugelkappen in vielen Punkten dem der Kreiszylinderschale ähnlich ist und zudem die theoretischen und experimentellen Untersuchungen über die lokal beanspruchten Kugelkappe geometrisch nichtlineares Tragverhalten berücksichtigen, werden auch diese Untersuchungen beschrieben und deren Ergebnisse im Vergleich dargestellt.

Am Beispiel der Kugelkappe unter einer im Scheitel angreifenden Einzellast wird das Tragverhalten in Abhängigkeit vom Schlankheitsgrad $\lambda$, vom bezogenen Stutzendurchmesser $d^{*}$ und vom $R / t$-Verhältnis grundlegend mit der FE-Methode untersucht. Der Vergleich des Tragverhaltens der Kugelkappe mit dem einer elastisch gebetteten Kreisplatte und eines Stabzweischlages zeigt ein analoges Tragverhalten dieser Strukturen in Abhängigkeit von den Geometrieparametern. Die Ergebnisse aus eigenen numerischen Berechnungen werden durch Vergleiche mit experimentellen und analytischen Ergbenissen verifiziert. Die numerische Nachberechnung von Versuchsergebnissen zeigt aber auch, dass die Ausführung der Lasteinleitungsstelle einen weitaus größeren Einfluss hat, als in vielen theoretischen und experimentellen Arbeiten angenommen wurde.

Wie Studien mit der FE-Methode an Kreiszylinderschalen unter radialen Einzellasten zeigen, können die Zusammenhänge zwischen dem Tragverhalten 
der Kugelkappe und den Parametern $\lambda, d^{*}$ und $R / t$ auch auf die Kreiszylinderschale übertragen werden. Dabei ist der Schlankeitsgrad $\lambda$ der Kugelkappe dem bezogenen Randabstand $L^{*}$ der Lasteinleitungsstelle der Kreiszylinderschale äquivalent. Der lokale Charakter des Problems wird dadurch bestätigt, dass die Abklinglängen der Verformungen und Spannungen kleiner als $10 \sqrt{R t}$ sind. Das bedeutet auch, dass der Einfluss der Randbedingungen auf das Last-Verformungsverhalten der Kreiszylinderschalen sehr gering ist und in den Berechnungen stets von einer gelenkigen Lagerung (RB2) ausgegangen werden kann. Bei gefüllten Kreiszylinderschalen wirkt sich der Radialdruck stabilisierend auf das Tragverhalten aus. Beim Vergleich der Ergebnisse eigener FE-Berechnungen mit den Ergebnissen aus experimentellen, analytischen und numerischen Untersuchungen zeigt sich eine gute Übereinstimmung. Die Abweichungen zwischen experimentellen und numerischen Ergebnissen sind dadurch zu erklären, dass die Messpunkte in den Versuchsbeschreibungen nicht ausreichend genau dokumentiert sind. Die Vergleichsberechnungen zeigen zudem, dass die Abschätzung der Spannungen und Verformungen mit analytischen Methoden gegenüber den numerischen Ergebnissen weit auf der sicheren Seite liegt und damit äußerst unwirtschaftlich ist.

In den numerischen Serienberechnungen werden Kreiszylinderschalen untersucht, deren $R / t$-Verhältnisse zwischen 400 und 5000 liegen. Die untersuchten bezogenen Stutzendurchmesser $d^{*}$ umfassen einen Bereich von 0,5 bis 3,5 bei einem bezogenen Randabstand $L^{*}$ zwischen 10 und 80. Es werden Kreiszylinderschalen unter Einwirkung radialer Einzellasten $P_{x}$, Momentenbelastung $M_{\varphi}$ in Umfangsrichtung und $M_{z}$ in Meridianrichtung unter Berücksichtigung geometrisch und physikalisch nichtlinearer Effekte untersucht. Es zeigte sich, dass unabhängig vom Lastfall das Tragverhalten der Kreiszylinderschale wesentlich vom bezogenen Stutzendurchmesser $d^{*}$ beeinflusst wird. Das Tragverhalten von Kreiszylinderschalen, die durch eine radiale Einzellast $P_{x}$ oder ein Moment $M_{z}$ in Meridianrichtung beansprucht werden, wird zudem vom bezogenen Randabstand $L^{*}$ beeinflusst. Im Lastfall $M_{\varphi}$ hat $L^{*}$ dagegen keinen Einfluss auf das Tragverhalten. Die Kombination aus $R / t$-Verhältnis und Streckgrenze $f_{y}$ des Werkstoffes wird mit dem Faktor $\eta$ erfasst. Die Abminderung des Tragverhaltens der Kreiszylinderschalen im plastischen Bereich steht in direktem Zusammenhang mit $\eta$. Bei Kreiszylinderschalen, die durch eine radiale Einzellast $P_{x}$ oder ein Moment $M_{\varphi}$ in Umfangsrichtung beansprucht werden, wirkt sich das plastische Werkstoffverhalten nur dann auf das Tragverhalten aus, wenn $\eta<1,2$ ist. Bei Belastung durch ein Moment $M_{z}$ in Meridianrichtung erfährt das kritische Moment im betrachteten Bereich keine Abminderung durch Plastizierung. Bei allen betrachteten Lastfällen ist der Einfluss des bezogenen Randabstandes $L^{*}$ auf 
das plastische Tragverhalten im plastischen Bereich sehr gering. Der Einfluss des bezogenen Stutzendurchmessers $d^{*}$ auf die kritische Last $P_{x, c r i t, G M N A}^{*}$ und das kritische Moment $M_{\varphi, c r i t, G M N A}^{*}$, kann durch eine konservative Abgrenzung des plastischen Bereichs abgedeckt werden (vgl. Abbildung 6.22 und Abbildung 6.39). Die Grenze zwischen diesen beiden Bereich ist folglich nur eine Funktion von $R / t$ und der Streckgrenze $f_{y}$ des Werkstoffes. In Abbildung 9.1 ist diese Grenzkurve für Kreiszylinderschalen unter Einzellast $P_{x}$ oder Moment $M_{\varphi}$ in Umfangsrichtung in Abhängigkeit von der Streckgrenze $f_{y}$ des Werkstoffes und des $R / t$-Verhältnisses angegeben. D.h. die kritischen Lasten und Momente von Kreiszylinderschalen, die unterhalb dieser Grenzkurve liegen, erfahren eine Abminderung infolge der Plastizierungen. Die kritischen Momente von Kreiszylinderschalen, die durch ein Moment $M_{z}$ in Meridianrichtung beansprucht werden, erfahren dagegen keine Abminderung durch Plastizierungen, da diese erst im Bereich großer Verformungen auftreten.

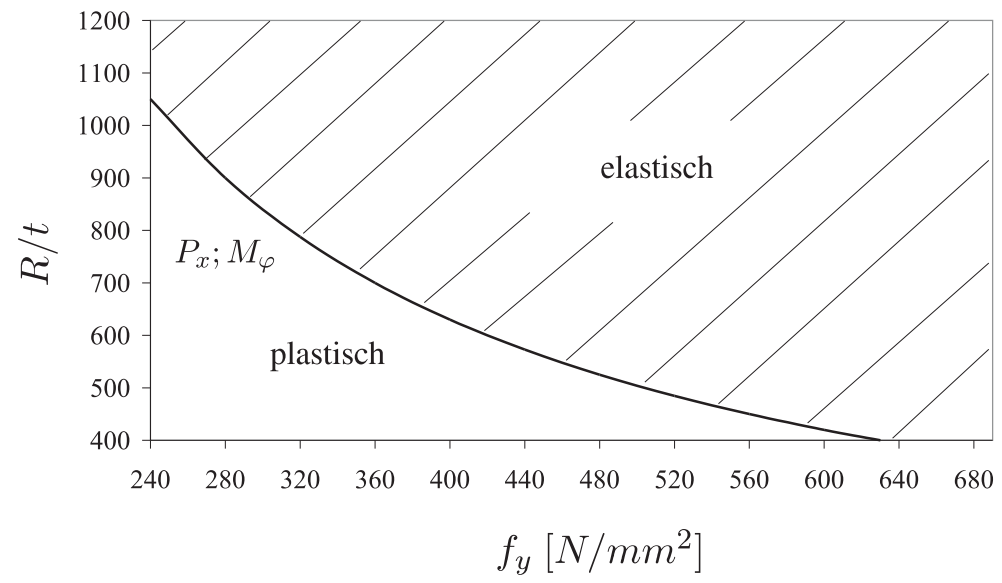

Abbildung 9.1: Elastische und plastische Bereiche der Kreiszylinderschalen unter radialer Einzellast oder Momentenbelastung in Umfangsrichtung

In den Untersuchungen über die scheibenförmig verstärkten Kreiszylinderschalen wird die Dicke der Verstärkungsmaßnahme als effektive Blechdicke $t_{v, \text { eff }}$ nach Gleichung 7.2 erfasst. Die effektive Blechdicke $t_{v, \text { eff }}$ berücksichtigt bereits den Unterschied zwischen Blechen, die in das Mantelblech eingesetzt oder auf das Mantelblech gesetzt sind. Die Exzentrizität der Bleche zum Mantelblech spielt eine untergeordnete Rolle und wird in den Berechnungen vernachlässigt. Die Abmessungen der Verstärkungsbleche sind an die Forderungen geknüpft, dass die 
Spannungen im unverstärkten Mantelblech immer kleiner sind, als die Spannungen im verstärkten Bereich. Aus kontruktiven Gesichtspunkten wird das Verhältnis des Verstärkungsblechdurchmessers zum Stutzendurchmeser auf $d_{v} / d_{r}<2,0$ und das Blechdickenverhältnis $t_{v, \text { eff }} / t$ auf maximal 2,0 beschränkt. Die Dimensionierung der Verstärkungsbleche beruht auf der Annahme geometrischer und physikalischer Linearität und kann mit den in Tabelle 9.1 aufgeführten Diagrammen erfolgen. Dort sind auch die Gleichungen zur Ermittlung der Spannungssteigerung $\sigma_{v e r}^{*} / \sigma_{\max , i}^{*}$ aufgeführt. Die maximalen Spannungen $\sigma_{\max , i}^{*}$ am Stutzenanschluss der unverstärkten Kreiszylinderschale können mit den in Tabelle 9.2 aufgeführten Gleichungen ermittelt werden.

\begin{tabular}{|l|l|l|}
\hline Lastfall $i$ & $\left(d_{v} / d_{r}\right)_{\min }$ & $\sigma_{v e r}^{*} / \sigma_{\max , i}^{*}$ \\
\hline$P_{x}$ & Abb. 7.9 & $1,16\left(\frac{t}{t_{v, e f f}}\right)-0,16$ \\
\hline$M_{\varphi}$ & Abb. 7.29 & $0,64\left(\frac{t}{t_{v, \text { eff }}}\right)+0,4\left(\frac{t}{t_{v, \text { eff }}}\right)-0,04$ \\
\hline$M_{z}$ & Abb. 7.43 & $1,73\left(\frac{t_{v, \text { eff }}}{t}\right)-0,73$ \\
\hline
\end{tabular}

Tabelle 9.1: Gleichungen und Bemessungsdiagramme zur Ermittlung der Spannungssteigerung durch Verstärkungsbleche und des Durchmesserverhältnisses $\left(d_{v} / d_{r}\right)_{\min }$

\begin{tabular}{|l|l|}
\hline Lastfall $i$ & $\sigma_{\max , i}^{*}$ \\
\hline$P_{x}$ & $-0,5 d^{*}+2,3 \sqrt{d^{*}}-3,2$ \\
\hline$M_{\varphi}$ & $0,69 \sqrt{d^{*}}-2,1$ \\
\hline$M_{z}$ & $0,015\left(d^{*}\right)^{2}+0,02 d^{*}-1,73$ \\
\hline
\end{tabular}

Tabelle 9.2: Gleichungen zur Ermittlung der maximalen Spannungen im unverstärkten Mantelblech

Im linearen Bereich der Last-Verformungskurven haben die Kreiszylinderschale eine Anfangssteifigkeit $c_{k}^{*}$. Diese Anfangssteifigkeit kann in Abhängigkeit von den Abmessungen des Verstärkungsbleches $t_{v, \text { eff }} / t$ und $d_{v} / d_{r}$ mit den in Tabelle 9.3 aufgeführten Gleichungen und Diagrammen ermittelt werden.

Die kritischen Lasten $S_{i, c r i t, k}^{*}$ und Grenzgrößen $\bar{S}_{i, \max , M N A}$ verstärkter und unverstärkter Kreiszylinderschalen können unter Berücksichtigung geometrischer und/oder physikalischer Nichtlinearitäten mit den in Tabelle 9.4 zusammengefassten Bemessungsdiagrammen und Gleichungen ermittelt werden. Die maximale 


\begin{tabular}{|l|l|}
\hline$c_{P_{x}}^{*}=c_{0, P_{x}}^{*} \cdot\left(L^{*}\right)^{\chi}$ & mit Abb. 7.19 bis Abb. 7.22 \\
\hline$c_{M_{\varphi}}^{*}$ & aus Abb. 7.35 und 7.36 \\
\hline$c_{M_{z}}^{*}=c_{0, M_{z}}^{*}+\frac{\omega}{L^{*}}$ & mit Abb. 7.51 bis 7.54 \\
\hline
\end{tabular}

Tabelle 9.3: Gleichungen und Bemessungsdiagramme zur Bestimmung der Anfangssteifigkeit

Verschiebung oder Verdrehung, die auftritt, wenn die kritische Last erreicht wird, ist dort ebenfalls angegeben.

Für die Grenzen des geometrisch und physikalisch linearen Bereichs gilt:

- Geometrisch nichtlineare Effekte können für Lastgrößen $S_{i}^{*}$, die kleiner als $0,4 S_{i, c r i t, k}^{*}$ vernachlässigt werden

- Plastisches Werkstoffverhalten beeinflusst das Tragverhalten der Kreiszylinderschale, wenn die Lastgrößen $\bar{S}_{i}$ größer als $0,4 \bar{S}_{i, \max , M N A}$ sind. 


\begin{tabular}{|c|c|}
\hline \multicolumn{2}{|l|}{ Lastfall $P_{x}$} \\
\hline $\begin{array}{l}P_{x, c r i t, G N A}^{*}=P_{0}^{*}+\frac{\psi}{L^{*}} \text { mit Abb. } 7.15 \text { bis } 7.18 \\
\bar{P}_{x, \text { max }, M N A}=2+4 d^{*} \\
\frac{P_{x, \text { crit }, \text { GMNA }}^{*}}{P_{x, \text { crit }, \text { GNA }}^{*}}=-0,245 \eta^{2}+0,66 \eta+0,56\end{array}$ & $u_{\text {grenz }}^{*} \approx 4$ \\
\hline \multicolumn{2}{|l|}{ Lastfall $M_{\varphi}$} \\
\hline $\begin{array}{l}M_{\varphi, c r i t, G N A}^{*} \text { mit Abb. } 7.33 \text { und } 7.34 \\
\bar{M}_{\varphi, \text { max }, M N A}=2,1\left(d^{*}\right)^{2}-1,4 d^{*}+2,6 \\
\frac{M_{\varphi, c r i t, G M N A}^{*}}{M_{\varphi, c r i t, G N A}^{*}}=-0,56 \eta^{2}+1,45 \eta+0,07\end{array}$ & $\alpha_{g r e n z}^{*} \approx 1$ \\
\hline \multicolumn{2}{|l|}{ Lastfall $M_{z}$} \\
\hline $\begin{array}{l}M_{z, \text { crit }, \text { GNA,ver }}^{*}=M_{z, 0}^{*}+\frac{\xi}{L^{*}} \text { mit Abb. } 7.47 \text { bis } 7.50 \\
\bar{M}_{z, \text { max }, M N A}=0,71\left(d^{*}\right)^{2}+1,94 d^{*}+0,5 \\
\frac{M_{z, \text { crit }, \text { GMNA }}^{*}}{M_{z, \text { crit }, \text { GNA }}^{*}}=1\end{array}$ & $\alpha_{\text {grenz }}^{*} \approx 1$ \\
\hline
\end{tabular}

Tabelle 9.4: Zusammenstellung der kritischen Lasten und Grenzlasten 


\section{Schrifttum}

[1] REISSNER, E.: Stresses and small displacements of shallow spherical shells. I. In: Journal of mathematics and physics 25 (1946), S. 80-85

[2] REISSNER, E.: Stresses and small displacements of shallow spherical shells. II. In: Journal of mathematics and physics 26 (1947), S. 279-300

[3] REISSNER, E.: On axisymmetrical deformations of thin shells of revolution. In: Proceedings of symposia in applied Mathematics Bd. 3, 1950, S. 27-52

[4] BiJlaARD, P.P.: Computation of the stresses from local loads in spherical vessels or pressure vessel heads. In: Welding research council bulletin 34 (1957)

[5] BiJlaARD, P.P: Local stresses in spherical shells from radial or moment loadings. In: Welding journal 36 (1957), S. 240-243

[6] BiJlaARD, P.P: Stresses in a spherical vessel from radial loads acting on a pipe. In: Welding research council bulletin 49 (1959), S. 1-30

[7] BiJllaARD, P.P.: Stresses in a spherical vessel from external moments acting on a pipe. In: Welding research council bulletin 49 (1959), S. 31-62

[8] Wichman, K.R. ; Hopper, A.G. ; Mershon, J.L.: Local Stresses in Spherical and Cylindrical Schells due to External Loadings. In: Welding research council bulletin 107 (1979)

[9] BiezEno, C.B.: Über die Bestimmung der Durchschlagkraft einer schwachgekrümmten, kreisförmigen Platte. In: Zeitschrift für angewandte Mathematik und Mechachnik 15 (1935), S. 10-22

[10] ChiEn, W.Z. ; HU, H.C.: On the snapping of a thin spherical cap. In: Proceedings 9th international congress of applied mechanics 6 (1956), S. 309-337

[11] Ashwell, D.G.: On the large deflexion of a spherical shell with an inward point load. In: Proceedings of the I.U.T.A.M symposium on the theory of thin elastic shells, Delft, 1959, S. 43-63 
[12] Evan-Iwanowski, R.M. ; Cheng, H.S. ; Loo, T.C.: Experimental investigation of deformations and stability of spherical shells subjected to concentrated load at the apex. In: Proceedings of the fourth U.S. national congress of applied mechanics, 1962, S. 563-575

[13] ARCHER, R.R.: On the numerical solution of the nonlinear equations for shells of revolution. In: Journal of mathematics and physics XLI (1965), Nr. 3, S. 165-178

[14] Mescall, J.F.: Large deflections of spherical shells under concentrated loads. In: Transactions of the ASME (1965), S. 936-938

[15] Penning, F.A. ; Thurston, G.A.: The stability of shallow spherical shells under concentrated load / National aeronautics and space administration NASA. 1965 ( CR-265). - Forschungsbericht

[16] SANDERS, J.L.: Nonlinear theories of thin shells / Harvard University. 1961 (10). - Forschungsbericht

[17] Penning, F.A.: Experimental buckling modes of clamped shallow shells under concentrated load. In: Journal of applied mechanics 33 (1966), S. 297-304

[18] FITCH, J.R.: The buckling and post-buckling behavior of spherical caps under concentrated load. In: International journal of solids and structures 4 (1968), S. 421-446

[19] RoARK, R.J.: The strength and stiffness of cylindrical shell under concentrated loading. In: Journal of applied mechanics 57 (1935), S. A147 - A152

[20] Schoessow, G.J. ; Kooistra, L.R: Stresses in a cylindrical shell due to nozzle or pipe connection. In: Journal of applied mechanics 12 (1945), S. A107-A112

[21] YUAN, S.W.: Thin cylindrical shells subjected to concentrated loads. In: Quarterly of applied mathematics 4 (1946), Nr. 1, S. 13-26

[22] Donell, J.H.: Stability of thin-walled tubes under torsion / NACA. Washington, D.C, 1933 ( 479). - Technical report

[23] Yuan, S.W. ; TING, L.: On radial deflections of a cylinder subjected to equal and opposite concentrated radial loads. In: Journal of applied mechanics 24 (1957), S. 278-282

[24] FLÜGGE, W.: Statik und Dynamik der Schalen. Springer-Verlag, 1934 
[25] BiJlaARD, P.P.: Stresses from local loadings in cylindrical pressure vessels. In: Transaction of the ASME 8 (1955), S. 805-816

[26] BiJlaARD, P.P.: Stresses from radial loads in cylindrical pressure vessels. In: Welding journal 33 (1954), S. 615-623

[27] BijlaARD, P.P: Stresses from radial loads and external moments in cylindrical pressure vessels. In: Welding journal 34 (1955), S. 608-617

[28] CRANCH, E.T.: An experimental investigation of stresses in the neighborhood of attachments to a cylindrical shell. In: Welding research council bulletin 60 (1960), S. 1-44

[29] Mershon, J.L ; Mokhtarian, K. ; Ranjan, G.V. ; Rodabaugh, E.C.: Local stresses in spherical and cylindrical shells due to external loadings on nozzles - supplement to WRC bulletin No. 107. In: Welding research council bulletin 297 (1987)

[30] Steele, C.R. ; STEELE, M.L.: Reinforced oppenings in largel pressure vessels: Effect of nozzle wall thickness / SHELLTECH. Stanford, California, 1981 ( 81-5). - Forschungsbericht

[31] Steele, C.R. ; Steele, M.L.: Stress analysis of nozzles in cylindrical vessels with external load. In: Journal of pressure vessel technology 105 (1983), S. 191-200

[32] SteEle, C.R.: Reinforced openings in large pressure vessels / SHELLTECH. Stanford, California, 1983. - Forschungsbericht

[33] Steele, C.R. ; Steele, M.L.: Reinforced openings in large steel pressure vessels:Solution of large openings / SHELLTECH. Stanford, California, 1984 ( 84-1). - Forschungsbericht

[34] FlÜGge, W. ; CONRAD, D.A.: Thermal singularities for cylindrical shells. In: Proceedings of the third U.S. national congress of applied mechanics, 1958, S. 321-328

[35] SAnders, J.; Lyell, J. ; Simmonds, J.G.: Concentrated forces on shallow cylindrical shells. In: ASME journal of applied mechanics 37 (1970)

[36] CBI (Hrsg.): Experimental testing programm for nozzle connections in cylindrical shells. Chicago bridge and iron company, 1979 ( 74-9453) 
[37] Gwaltney, R.C. ; Corum, J.M. ; Bolt, S.E. ; Bryson, J.W.: Experimental stress analysis of cylinder-to-cylinder shell models and comparisons with theoretical prediction. In: Journal of pressure vessel technology (1976), S. 283-290

[38] MoRley, L.S.D.: An improvement on Donnell's appromimation for thinwalled circular cylinders. In: Quarterly journal of mechanics and applied mathematics 12 (1959), S. 89

[39] MORLEY, L.S.D.: The thin-walled circular cylinder subjected to concentrated radial loads. In: Quarterly journal of mechanics and applied mathematics 13.1 (1960), S. 24-37

[40] Kupfer, W.: Spannungen in Zylinderschalen mit radialen Stutzen infolge äußerer Belastungen nach der Elastizitätstheorie. In: Stahlbau (1990), S. 237-245

[41] Wordsworth, A.C.: Stresses in cylindrical pressure vessels due to local loads. In: Structural integrity assessment, Elsevier Applied Science (1992), S. $299-401$

[42] DeKKer, C.J.: Comparison of local load stress calculation methods for nozzles on cylinders. In: The international journal of pressure vessels and piping 59 (1994), S. 203-213

[43] Rudolph, J.: Nachweis der Ermüdungsfestigkeit von Behälter-StutzenVerbindungen unter Einwirkung von Innendruck und Rohrleitungslasten. VDI-Verlag, 1997 ( 206)

[44] Joost, H.: Nachweis der Tragfähigkeit von Behälter-Stutzen-Verbindungen unter Einwirkung von Innendruck und Rohrleitungslasten, Fachbereich Chemietechnik der Universität Dortmund, Diss., 1998

[45] Rauth, M.: FE-integrierendes Berechnungskonzept für den Ermüdungsfestigkeitsnachweis von Behälter-Stutzen-Verbindungen unter Berücksichtigung von Innendruck und Rohrleitungslasten, Fachbereich Chemietechnik der Universität Dortmund, Diss., 2002

[46] Gillie, M. ; RotTer, J.M.: The effects of patch loads on thin-walled steel silos. In: Thin-walled structures 40 (2002), S. 835-852

[47] SonG, C.Y.: Effects of patch loads on structural behavior of circular flatbottomed steel silos. In: Thin-walled Structures 42 (2004), S. 1519-1542 
[48] Guggenberger, W. ; Greiner, R. ; Rotter, J.M.: The behaviour of locally-supported cylindrical shells: unstiffened shells. In: Journal of constructural steel research 56 (2000), S. 175-97

[49] Gillie, M. ; Holst, J.M.F.G. ; MÜNCH, M. ; RotTer, J.M.: Behaviour of silos supported on discrete brackets. In: Journal of structural stability and dynamics 2 (2002), S. 45-62

[50] Gillie, M. ; Holst, J.M.F.G.: Structural behaviour of silos supported on discrete, eccentric brackets. In: Journal of constructural steel research 59 (2003), S. 887-910

[51] ANSYS Release 7.1; Documentation, 2004

[52] RIKS, E.: The application of Newton's method to the problem of elastic stability. In: Journal of applied mechanics 39 (1972), S. 1060-1066

[53] RIKS, E.: An incremental approach to the solution of snapping and buckling problems. In: International journal of solids structures 15 (1979), S. 529551

[54] CRISfield, M.A.: A fast incremental/iterative solution procedure that handles snap-through. In: Computers and structures 13 (1980), S. 55-62

[55] Institut für Statik und Mechanik der Universität Hannover: ROTASS 1.34, Programm zur Berechnung rotationssymmetrischer Schalen. 2005

[56] PflüGer, A.: Elementare Schalenstatik. 4. Auflage. Springer-Verlag, 1967 


\section{Zitierte Normen und Richtlinien}

AD 2000-Merkblatt B9 Oktober 2000. Ausschnitte in Zylindern, Kegeln und Kugeln, Verband der Technischen Überwachungs-Vereine e.V.

AD 2000-Merkblatt S3/9 September 2001. Allgemeiner Standsicherheitsnachweis für Druckbehälter; Behälter mit Stutzen unter Zusatzbelastung, Verband der Technischen Überwachungs-Vereine e.V.

API Standard Oktober 1998. Welded steel tanks for oil storage, American petroleum institute.

BS 5500 Januar 1985. Specification for unfired, fusion welded pressure vessels, British standard institution.

DIN 1055 Teil 6 März 2005. Einwirkungen auf Tragwerke - Einwirkungen auf Silos und Flüssigkeitsbehälter. Deutsches Institut für Normung.

DIN 4119 Teil 2 Februar 1980. Oberirdische zylindrische FlachbodenTankbauwerke aus metallischen Werkstoffen; Berechnung, Deutsches Institut für Normung.

DIN EN 14015 Februar 2005. Auslegung und Herstellung standortgefertigter, oberirdischer, stehender, zylindrischer, geschweißter Flachboden-Stahltanks für die Lagerung von Flüssigkeiten bei Umgebungstemperatur und höheren Temperaturen, Deutsches Institut für Normung.

DIN 18800 Teil 4 November 1990. Stahlbauten - Stabilitätsfälle, Schalenbeulen, Deutsches Institut für Normung.

DIN V ENV 1993 Teil 4-2 Mai 2002. Bemessung und Konstruktion von Stahlbauten. Silos, Tankbauwerke und Rohrleitungen - Tankbauwerke, Deutsches Institut für Normung. 
DIN V ENV 1993 Teil 1-6 Mai 2005: Allgemeine Bemessungsregeln; Ergänzende Regeln für Schalenkonstruktionen; Deutsche Fassung ENV 1993-1-6:1999, Deutsches Institut für Normung.

Richtlinie für die Herstellung von Flachbodentanks mit besonderen Anforderungen, Vereinbarung 2002/1 zwischen FDBR und VdTÜV, 2002. 